UNIVERSIDADE DE SAO PAULO

INSTITUTO DE FISICA E QUIMICA DE SAO CARLOS

"Caracterizaçao do LiNbos:Er"s como meio ativo para lasers de estado solido através dos espectros de absorçăomissão polarizada e medida dos. tempos de vida de luminescencia".

Débora Marcondes Bastos Pereira Milori

Orientador: Prof. Dr. Máximo Siu Li Dissertação apresentada ao Instituto de Fisica e Quimica de sao Carlos para obtençåo do titulo de MESTRE EM FISICA BÁSICA

Departamento de Fisica e Ciencia dos Materiais Såo Carlos - São Paulo 
MEMBROS DA COMISSÃO JULGADORA DA DISSERTAÇÃO DE MESTRADO DE Debora Marcondes Bastos Pereira Milori APRESENTADA

AO INSTITUTO DE FISICA E QUIMICA DE SÃO CARLOS, DA UNIVERSI DADE DE SÄO PAULO, EM 20 DE setembro DE 1989.

COMISSÃO JULGADORA:
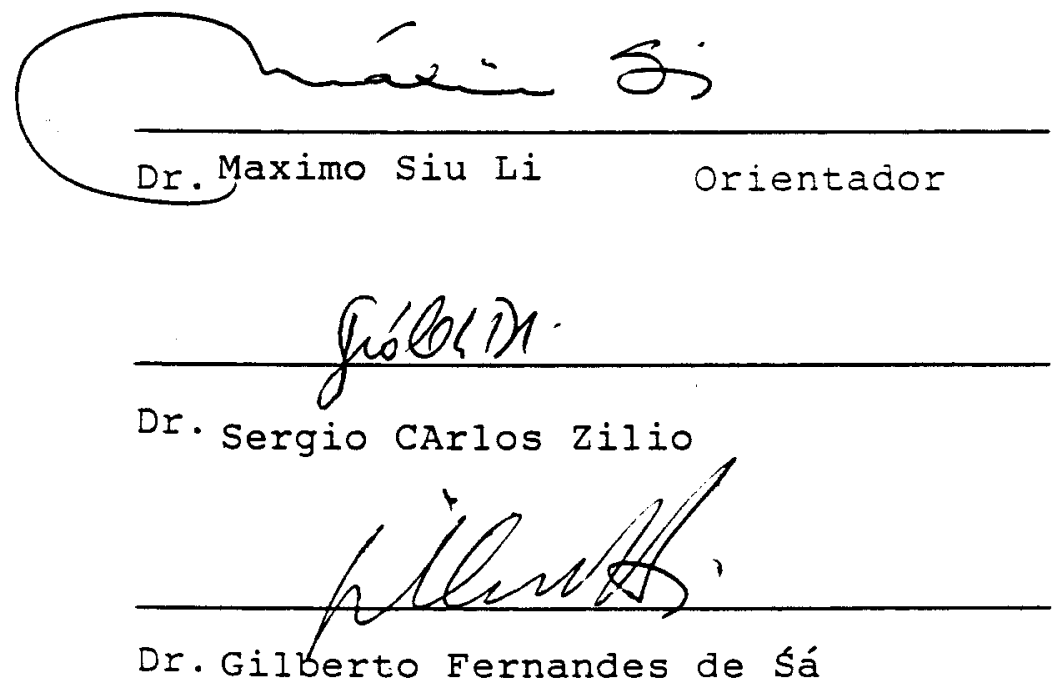
Nao quero ROSAS, desde que haja rosas. Quero-as so quando nå as possa haver. Que hei de fazer das coisas Que qualquer måo pode colher?

\section{Fernando Pessoa}


RESUMO

Este trabalho consiste na caracterizaçao otica de mono cristais de $\mathrm{LiNbO}_{3}: \mathrm{Er}^{+\mathrm{J}}$, visando a possibilidade deste sistema ser meio ativo para laser. Os resultados obtidos para absorção e emissão foram satisfatorios. sendo bastante compativeis com os do modelo teórico adotado. O tempo de vida de luminescência das transiçסes ${ }^{4} \mathrm{~S}_{3 / 2} \rightarrow{ }^{4} \mathrm{I}_{1 \mathrm{~J} / 2=}$ e ${ }^{4} \mathrm{~F}_{9 / 2} \rightarrow{ }^{4} \mathrm{I}_{1=/ 2}$ foi medido, à temperatura ambiente e de nitrogénio liquido. mostrando que elas săo possiveis candidatas a transiçð̋es laser.

Também foi feito um estudo sobre o comportamento das concentraçós das impurezas indesejáveis $\mathrm{OH}^{-}$e $\mathrm{Fe}^{2+}$, através de tratamento térmico. O controle dessas concentrações foi feito atraves das técnicas de RPE e absorção ótica e os resultados mostraram que é perfeitamente possivel eliminar tais impurezas de uma forma totalmente controlada. 


\section{ABSTRACT}

This work consists in the optical characterization of LiNbO : Er ${ }^{+3}$ single crystals, aiming to verify the possibility of this system being an active media for laser. The absorption and emission results were satisfactory, being compatible enough with the theoretical model adopted. The luminescence lifetime of the ${ }^{4} \mathrm{~S}_{5 / z} \rightarrow{ }^{4} \mathrm{I}_{15 / z}$ and ${ }^{4} \mathrm{~F}_{9 / 2} \rightarrow{ }^{4} \mathrm{I}_{13 / z}$ transitions was measured at the ambient and liquid nitrogen temperature. showing that they are possible candidates for laser transitions.

The behaviour of the undesirable $\mathrm{OH}^{-}$and $\mathrm{Fe}^{2+}$ impurities was also studied through thermal treatment. The control of these concentrations was done using the EPR and optical absorption techniques and the results showed it is possible to eliminate such impurities in a totally controlled way. 


Aos meus pais, Sebastiá e Enoi e
ao Marcius, meu marido, meu
agradecimento especial, pelo
incentivo, apoio e compreensá.




\section{AGRADECIMENTOS}

Ao prof. Máximo Siu Li pela amizade, orientaçăo e dedicaçăo durante a realização deste trabalho.

A profa. Maria Cristina Terrile pela gentileza e dedicaça durante as discussరes e pelas valiosas sugestoes.

Aos profs. Vanderlei Salvador Bagnato e Liderio Ioriatti pelas sugestốs e pela amizade.

Aos profs. J.P. Andreeta e H. Gallo pelos cristais gentilmente cedidos.

Ao prof. Otaciro Rangel Nascimento e profa. Maria Cristina Terrile pelo apoio e auxilio nas medidas de RPE e pela amizade.

Aos técnicos Gilberto e Paulo pela boa vontade com que colaboraram durante o perłodo de realizaçăo das montagens e medidas experimentais.

Ao prof. Eduardo M. Belo (EESC-USP) e sua esposa Heloisa pela amizade e colaboração.

Aos orgãos financiadores : FAPESP, CNPq e FINEP.

Enfim, a todos os colegas do DFCM. 
ÍNDICE

RESUMO

ABSTRACT

AGRADECIMENTOS

INDICE

CAPITULO I - INTRODUÇAO

CAPI TULO I I - CONSIDERAÇOES GERAIS

$2.1 \quad-$ INTRODUÇAO $\ldots \ldots \ldots \ldots \ldots \ldots \ldots \ldots \ldots \ldots \ldots \ldots \ldots \ldots \ldots \ldots \ldots$

$2.2 \quad-$ TEORIA DO CAMPO CRISTALINO $\ldots \ldots \ldots \ldots \ldots \ldots \ldots$

2.3 - APLICACAO DA TEORIA DE GRUPOS PARA IDENTIFICAÇO DAS TRANSI ÇZES ELETRÔNICAS

2.3 .1 - "SPLITTING" DOS NIVEIS ELETRÔNICOS $\ldots \ldots \ldots \ldots \ldots 7$

2.3 .2 - NATUREZA E POLARIZACAO DAS TRANSIÇUES.......... 7

2.3.3 - REGRAS DE SELEÇAO DAS TRANSIÇOES $\ldots \ldots \ldots \ldots \ldots$. 8

2.3 .4 - INTESIDADE DAS TRANSIÇOES $\ldots \ldots \ldots \ldots \ldots \ldots \ldots \ldots \ldots$

2.4 - ESPECTRO DE ABSORGAO E EMISSAO DE TERRAS-RARAS EM CRISTAIS

2.4.1 - COEFICIENTES DE EINSTEIN PARA TERRAS-RARAS

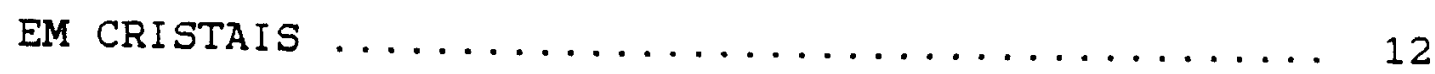

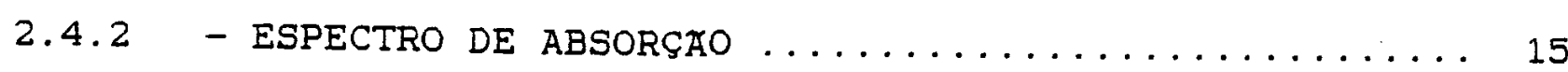

2.4 .3 - ESPECTRO DE EMISSAO

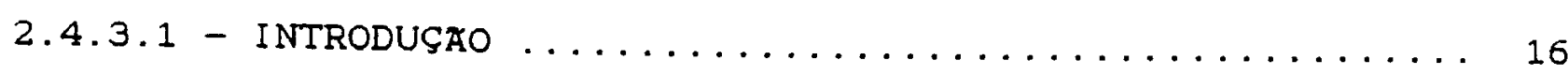

2.4.3.2 - EFICIÊNCIA QUÂNTICA E O TEMPO DE

VIDA dos Estados LUMinESCENTES ............. 19

2.5 - O CRISTAL LINbO= 
$2.5 .1-$ ESTRUTURA............................ 20

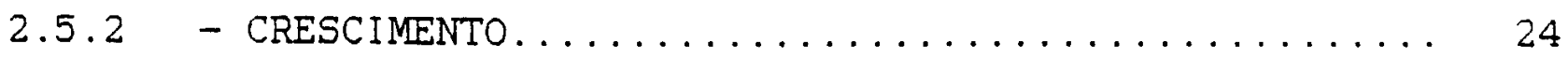

2.5.3 - INCORPORAÇO DE DOPANTES NUMA MATRIZ CRISTALINA... 25

2.5.4 - ALGUMAS PROPRIEDADES ÓTICAS DO LiNbO=

2.5 .4 .1 - ESPECTRO ÓTICO ........................ 26

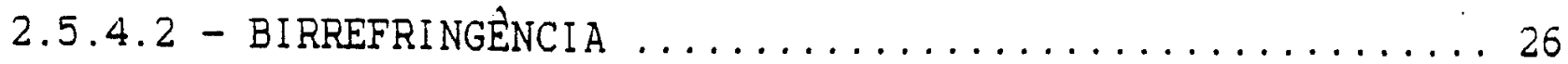

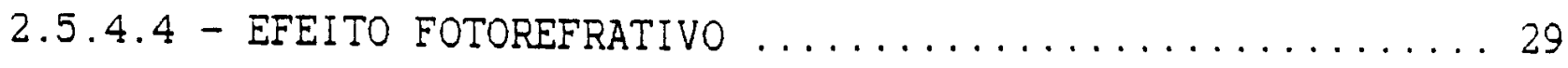

2.5 .5 - EFEITOS DE IMPUREZAS NAS PROPRIEDADES DO LINbO=

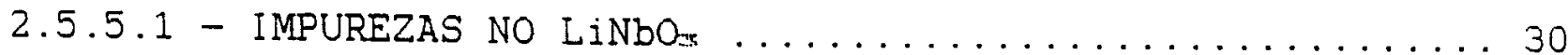

2.5 .5 .2 - PROPRIEDADES DO LINbO $=$ DOPADO COM FERRO ......... 31

2.5 .5 .3 - PROPRIEDADES DO LiNbO $=$ DOPADO $\mathrm{COM} \mathrm{OH}^{-} \ldots \ldots \ldots \ldots 32$

$2.6-O$ ION $\mathrm{Er}^{+3}$

2.6.1 - CARACTERISTICAS GERAIS DOS ÍONS DE TERRAS-RARAS ... 32

2.6 .2 - CARACTERISTICAS DO ERBIO $\ldots \ldots \ldots \ldots \ldots \ldots \ldots \ldots$

2.6 .3 - COMPORTAMENTO DO E ${ }^{+}=$ALGUMAS MATRIZES $\ldots \ldots \ldots \ldots 34$

2.7 - INTRODUÇAO TEÓRICA A TECNICA DE RESSONÂNCIA PARAMAGNETICA ELETRÔNICA

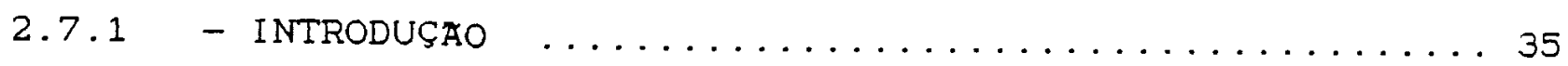

$2.7 .2-$ ESTRUTURA FINA $\ldots \ldots \ldots \ldots \ldots \ldots \ldots \ldots \ldots \ldots \ldots \ldots \ldots \ldots \ldots \ldots \ldots$

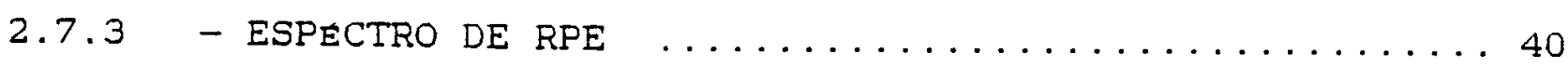

CAPITULO III - TECNICAS EXPERIMENTAIS

3.1 - PREPARACAO DAS AMOSTRAS

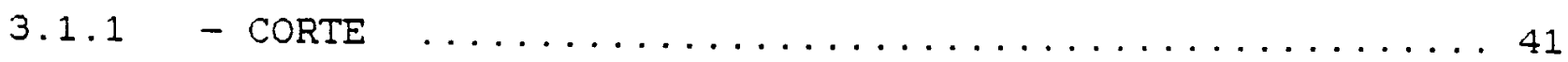

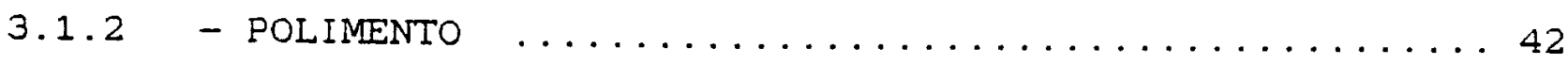

3.2 - DEWAR" UTILIZADO NAS MEDIDAS

A BAIXA TEMPERATURA $\ldots \ldots \ldots \ldots \ldots \ldots \ldots \ldots \ldots \ldots \ldots \ldots$ 


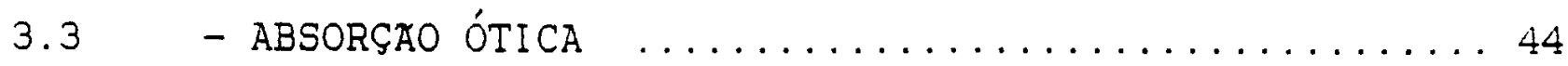

3.4 - RESSONÂNCIA PARAMAGNETICA ELETRÔNICA $\ldots \ldots \ldots \ldots 44$

3.5 - TRATAMENTO TERMICO

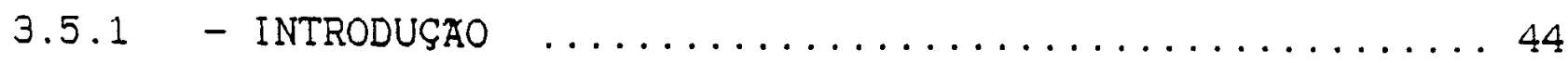

3.5 .2 - TRATAMENTO TERMICO NO AR $\ldots \ldots \ldots \ldots \ldots \ldots \ldots 45$

3.5.3 - TRATAMENTO TERMICO EM ATMOSFERA DE OXIGÊNIO $\ldots \ldots \ldots \ldots \ldots \ldots \ldots \ldots \ldots \ldots \ldots \ldots \ldots \ldots \ldots \ldots \ldots \ldots$

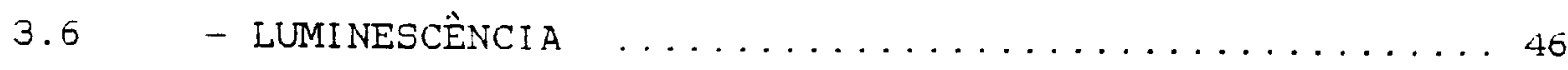

3.7 - MEDIDA DO TEMPO DE VIDA DOS ESTADOS

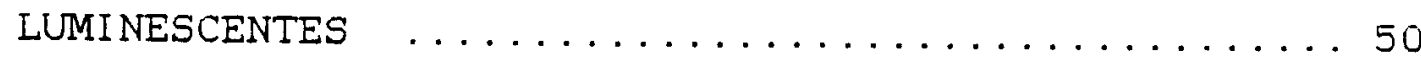

CAPITULO IV - RESULTADOS EXPERIMENTAIS

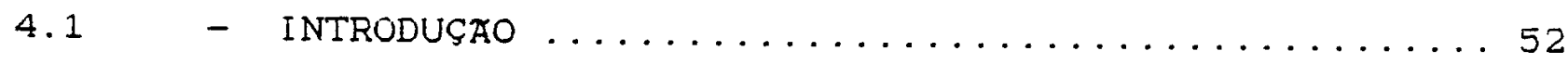

4.2 - TRATAMENTOS TERMi COS

4.2 .1 - TRATAMENTO TERMI CO NO AR $\ldots \ldots \ldots \ldots \ldots \ldots \ldots \ldots \ldots \ldots \ldots \ldots \ldots \ldots$

4.2 .2 - TRATAMENTO TERMiCO EM ATMOSFERA

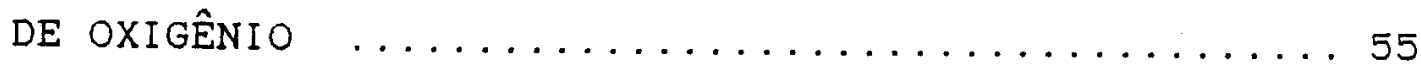

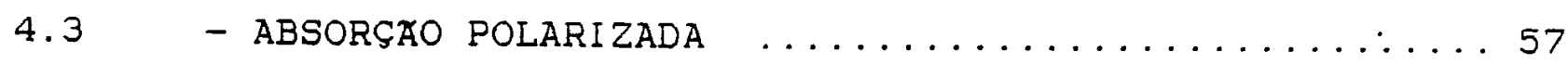

$4.4 \quad$ - EMISSAO POLARIZADA $\ldots \ldots \ldots \ldots \ldots \ldots \ldots \ldots \ldots \ldots \ldots \ldots \ldots \ldots \ldots$

4.5 - TEMPO DE VIDA DE LUMINESCÊNCIA $\ldots \ldots \ldots \ldots \ldots \ldots$

CAPITULO V - ANÁLISE E DISCUSSAO DOS RESULTADOS

$5.1 \quad$ - INTRODUÇAO $\ldots \ldots \ldots \ldots \ldots \ldots \ldots \ldots \ldots \ldots$. 
5.2 - AVALIAÇAO DOS EFEITOS DOS TRATAMENTOS

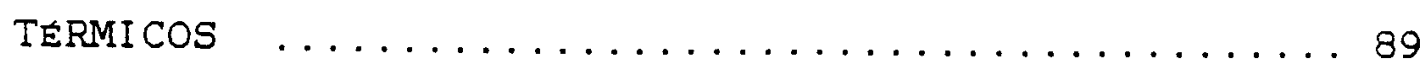

5.3 - ANÁLISE DO ESPECTRO DE ABSORGAO $\ldots \ldots \ldots \ldots \ldots \ldots$

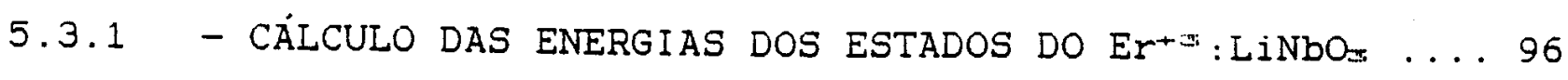

5.4 - ANÁLISE DOS ESPECTROS DE EMISSAO $\ldots \ldots \ldots \ldots \ldots 110$

5.5 - ANÁLISE DAS MEDIDAS DE TEMPO DE VIDA DE

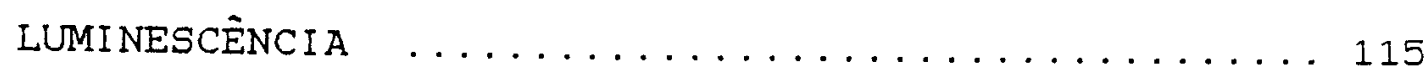

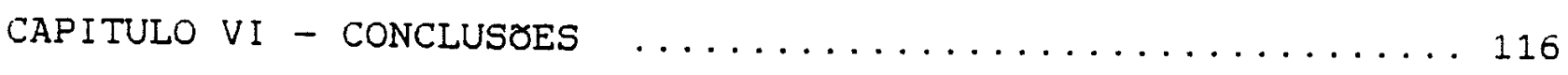

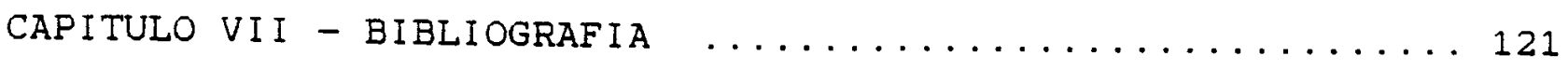

APÊND I CES 


\section{CAPITULO I - INTRODUÇAO}

Uma atrativa combinação de propriedades piezoeletricas e oticas tèm feito do LiNbO um dos materiais mais extensivamence estudados nos ultimos anos. Suas propriedades oticas dependem fortemente de impurezas introduzidas durante o crescimento e de tratamentos posteriores ao crescimento, tais como oxidaçăo, reduçăo e irradiaçåo. 10.20.27. $(16,12, \ldots, 21)$

o interesse do uso deste material dopado com terras-raras para meio ativo em lasers de estado solido vinha da possibilidade de juntar esta propriedade com as que ele possui naturalmente e produzir dispositivos integrados.

o primeiro resultado importante neste sentido foi obtido em 1967 por N.F. Evlanova: e coautores que observaram emissao estimulada de LiNbOs: $\mathrm{Nd}^{+}$. Em 1969 L.F. Johnson e A.A. Ballmanz= caracterizaram $\mathrm{LiNbO}: \mathrm{Nd}^{+3}, \mathrm{LiNbO}_{3}: \mathrm{Tm}^{-3}$ e LiNbOs:HO-3 como meios ativos para laser na região do infravermelho. Seis anos depois I.P. Kaminow e L.W. Stulz construiram um laser de LiNbO $\mathrm{z}^{-3}$ : cujo ganho nå era muito menor que o Nd:YAG, mas devido ao efeito fotorefrativo funcionava a $165^{\circ} \mathrm{C}$. Finalmente, em $1987^{\mathrm{A}}$. Cordova-Plazali e coautores construiram um laser de LiNbOz: $\mathrm{Nd}^{+\infty}$ : Mgo que funciona a temperatura ambiente e com este material foram feitas tres modalidades de laser : CW, com "Q-Switch" interno e auto-dobrador de frequencia.

A viabilizaçao de laser de niobato de litio as propriedades oticas deste cristal e o fato de possuirmos estes cristais 
com boa qualidade nos levou ao interesse em observar a ação laser de outras terras-rara nesta matriz e posteriormente a construça do laser.

Este trabalho consiste em estudar a absorcăo e emissão

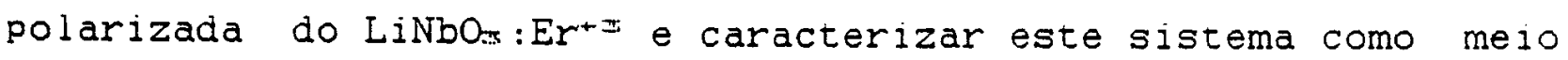
ativo para laser atraves de medidas de rempo de vida dos estados luminescentes.

No capitulo il fazemos uma revisao dos conceicos basicos usados na interpretaçăo dos resultados apresentados no capitulo IV e discutidos no capitulo $\mathrm{V}$. No capitulo II apresentamos as técnicas experimentais usadas e no capitulo VI tiramos conclusoes gerais do trabalho.

No apèndice $A$ apresentamos maiores informaçoes sobre a teoria dos operadores equivalentes. No apendice $C$ iornecermos as tabelas da teoria de grupos utilizadas para nossos calculos e no apendice $B$ fornecemos tabelas com os elementos de matriz dos operadores equivalentes de ordem 2.4 e 0 . 


\section{CAPITULO II - CONSIDERACOES GERAIS}

\subsection{INTRODUÇAO}

Este capituio esta dividido em sece partes : 2.2 Teoria do campo cristalino . 2.3 Aplicaça da teoria de grupos para identificaçăo de transiç̋es eletronicas . 2.4 Espectro de absorçăo e emissão de ions terras-raras em cristais. 2.50 cristal

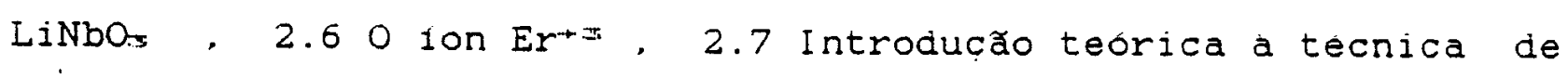
Ressonància paramagnetica eletronica e 2.8 incorporação de dopantes no LiNbOs. Com esta divisão pretendemos fazer uma abordagem geral dos principais topicos do problema, ou seja. a forma de resolver o problema teoricamente para avaliar as medidas experimentais . o estudo das propriedades oticas do LiNbo: que poderăo influir na açăo laser do cristal, o estudo da estrutura cristalina que nos dara a informação da simetria local que $\circ r^{+}$ fica sujeito quando entra na rêde, e finalmente um estudo do comportamento do ion Er ${ }^{+ \pm}$em outras matrizes.

\subsection{TEORIA DO CAMPO CRISTALINO}

Quando colocamos um ion num cristal, a simetria local do ion passa de esierica para a simetria da réde. Isto acontece devido as cargas dos lons vizinhos que geram um campo eletrico que interage com os elétrons mais energéticos da impureza e deforma a configuraçå de seus orbitais, o que gera um 
levantamento da degenerescencia dos niveis de energia . Nesta seç̧a vamos ver como tratar este problema.

o Ion livre tem uma Hamiltoniana composta peia energia cinetica de seus eletrons. interaça Coulumbiana dos eletrons com o nucleo. interaçăo Coulumbiana de eletron com eletron e interaçăo do spin do elétron ( $\overrightarrow{\boldsymbol{g}}$ ) com seu momentum angular $(\vec{i})$ :

$$
H_{0}=-\frac{h^{2}}{2 m} \sum_{1} \nabla_{1}^{2}+\sum_{1} \frac{Z_{\theta^{2}}^{2}}{r_{1}}+\frac{1}{2} \sum_{1+1} \frac{e^{2}}{r_{1} x^{2}}+\sum_{1} \xi_{i}(r) \vec{l}_{1} \cdot \vec{s}_{1}
$$

Existem ainda outros termos menos relevantes tais como: a interaçăo hiperfina entre o spin nuclear e o spin eletrónico e a interaçăo eletrostatica do momentum quadrupolar do nucleo com 0 elétron. mas estes termos nå serăo levados em conta neste trabalho por nåo trazerem informaçðes adicionais em relaçăo a caracterização do sistema como meio ativo para laser.

Se colocarmos este ion num cristal temos o potencial dos fons vizinhos e a Hamiltoniana passa a ser :

$$
\text { H }=H_{0}+\text { Hefimtal }
$$

Quando vamos perturbar os auto-valores da Ho com Heriatal. é importante saber se a perturbaçao é da mesma ordem de magnitude da repulsão eletrónica ou do termo spin orbita. Podemos ter tres casos :

$$
\begin{aligned}
& \text { 1. Heriatal }<E(r) \vec{I} \cdot \vec{g} \\
& \text { 2. E(r) } 1.9<\text { Herigtal }<e^{2} / r_{1} \\
& \text { 3. Horiatal... }<\text { e } e^{2} / r_{1}
\end{aligned}
$$

No primeiro caso se encaixam os lons de terras-raras, no segundo os elementos de transição e no terceiro os complexos covalentes. Por hora , estamos interessados no caso 1 , ou

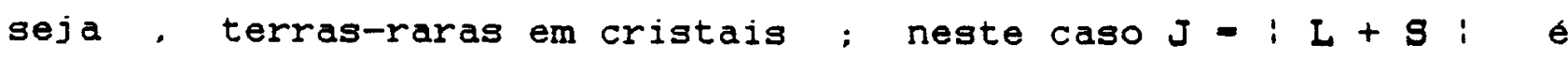


constante de movimento, logo e o numero quântico adequado para descrever o sistema.

Para qualquer envoltorio cristalino podemos estimar o potencial proximo do 10 em questao considerando cada ion vizinno como uma carga pontual . Se tomarmos como origem do sistema de coordenadas $\circ$ centro do ion de terra-rara, este potencial obedece a equaçăo de Laplace $\left(\nabla^{\text {FHeristal }}=0\right)$, pois as cargas que o produzem estå fora da regiăo de interesse, portanto. apresenta como soluçăo :

$$
\text { HeFistal }(r, \theta, \varnothing)-\sum_{1=m} A_{1}, m r^{1} Y_{1} m(\theta, \varnothing)
$$

A simetria de Herwtmi será a simetria local do lon dopante no cristal.

Escrever a Hamiltoniana do campo cristalino em termos de harmônicos estericos nos mostra que o problema e'soluvel, pois indica que $\mathrm{H}_{0}$ e Horrstal tem uma base comum mas trabalhar com as integrais envolvidas neste problema pode não ser muito agradavel, portanto a maneira mais indicada de atacar o probiema e atraves de operadores equivalentes.

A ideia de operadores equivalentes foi dada por Stevens ${ }^{\mathrm{a}}$. Este conceito consiste basicamente na substituiça das funçðes que descrevem o potencial por operadores construidos por combinaçбes de $J$. $J_{x} . J_{\searrow}$ e $J_{z}$ que possuam as mesmas propriedades da função. Por exemplo :

$\Sigma\left(3 z_{1}=-\gamma=\right) \equiv \alpha<r>\left[3 J_{2}=-J(J+1)\right]-\alpha<r<>O_{2}{ }^{0}$

A vantagem desta maneira de tratar o problema é que num sistema onde $J$ é uma constante de movimento conhecemos como $J$. $J_{x}$. Jx e Jx atuam em estados $\left|J, M_{x}, L, S\right\rangle$ e o problema fica reduzido a determinaçăo de constantes radiais $\left\langle m^{n}\right\rangle$, ou seja que dependem da simetria, e constantes que dependem apenas de $J$, as 
quais podem ser ajustadas por medidas experimentais. Os detalhes desta teoria estao no apendice A.

No apêndice $B$ temos as tabelas dos resultados dos elementos de matriz de operadores equivalentes pares para varios valores de J.

Agora podemos resolver o problema exatamente pois.

$\left\langle J, M_{J}, L, S: H i J, M_{J}, L, S\right\rangle \quad=\quad\left\langle J, M_{J}, L, S: H_{0}: J, M_{J}, L, S\right\rangle+$

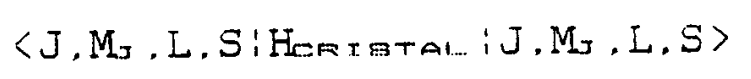

ou na base dos auto-estados do sistema.

$$
\left(\begin{array}{l}
E_{1} \\
E_{2} \\
\cdot \\
\cdot \\
E_{z J+1}
\end{array}\right)=\left(\begin{array}{c}
E_{O} \\
E_{0} \\
\cdot \\
\cdot \\
E_{0 S}
\end{array}\right)+\left(\begin{array}{c}
-E_{1} \\
-E_{2} \\
\cdot \\
\cdot \\
-E_{-I+1}
\end{array}\right)
$$

portanto diagonalizando Hefratal na base $U$ J.M.,L,S t temos 0 "splitting" e a separação entre os niveis de energia.

2.3 APLICACAO DA TEORIA DE GRUPOS PARA IDENTIFICACAO DE TRANSI CEES ELETRÔNICAS

Como vimos na seçăo 2.2 . o campo cristalino levanta a degenerescencia dos niveis de energia do ion de cerra-rara . As transições eletronicas entre estes niveis passam a ter regras de seleçăo e polarizaçao determinadas pela simetria local do ion. Nesta seça vamos tratar da teoria que nos iornece estas informaçðes.

Os detalhes sobre teoria de grupos podem ser encontrados nas referencias $[8,39]$. As tabelas comumente utilizadas nesta teoria para as simetrias envolvidas neste trabalho estao no apêndice $C$. 


\section{3 .1 "SPLITIING" DOS NIVEIS}

A perturbaca do campo cristaino e expressa por :

$$
\text { Hers atal. }-\sum_{i} \in V\left(r_{1}\right)
$$

No caso de campo iraco, que éo que nos interessa. j é a constante de movimento ( numero quántico adequado para descrever - sistema, e Heriaral perturba os estados do íon livre. Desta forma ocorre um splitting da representaçăo Ds em componentes, e cada uma destas componentes é uma representação irredutivel do grupo de operaçoes de simetria. as quais deixam Horarata invariante.

$$
\text { D. }=\sum_{1} \Gamma_{1}
$$

A degenerescencia do estado e dada pela dimensão de $\Gamma_{1}$. A forma com que Da vai se desdobrar na simetria do cristal e dada pela equaçăơ (9) :

$$
\chi\left(D_{J} \pm, R(\theta)\right)=\frac{\operatorname{SEN}(J+1 / 2) \theta}{\operatorname{SEN}(\theta / 2)}
$$

se $R(\theta)$ e uma rotação propria. Para rotaçós improprias temos.

$$
\begin{aligned}
& X\left(D_{J^{+}}, R(i \theta)\right)=\chi\left(D_{J^{ \pm}}, R(\theta)\right) \\
& \chi\left(D_{J^{-}}, R(i \theta)\right)=-\chi\left(D_{J^{ \pm}}, R(\theta)\right)
\end{aligned}
$$

Estes resultados ja estão tabelados ${ }^{\circ}$. No apendice $B$ temos as tabelas para as simetrias que vamos estudar neste trabalho.

\subsubsection{NATUREZA E POLARIZAÇAO DAS TRANSIÇOS}

Se um cristal possue um eixo otico, ou eixo de maior simetria (c), e de se esperar que os fenomenos ilsicos ocorram de forma diferente nas direços paralela e perpendicular a este eixo. 


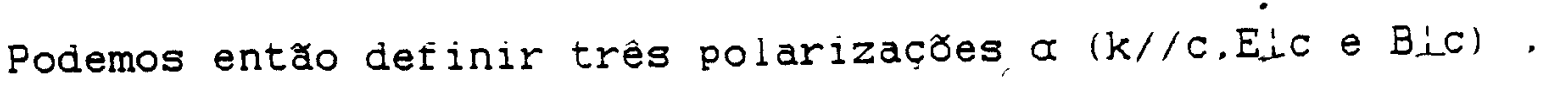
$\pi(k i C, E / / C$ e $B i C)$ e $\sigma(k i C, E i C$ e $B / / C)$, como mostra a figura 1 .

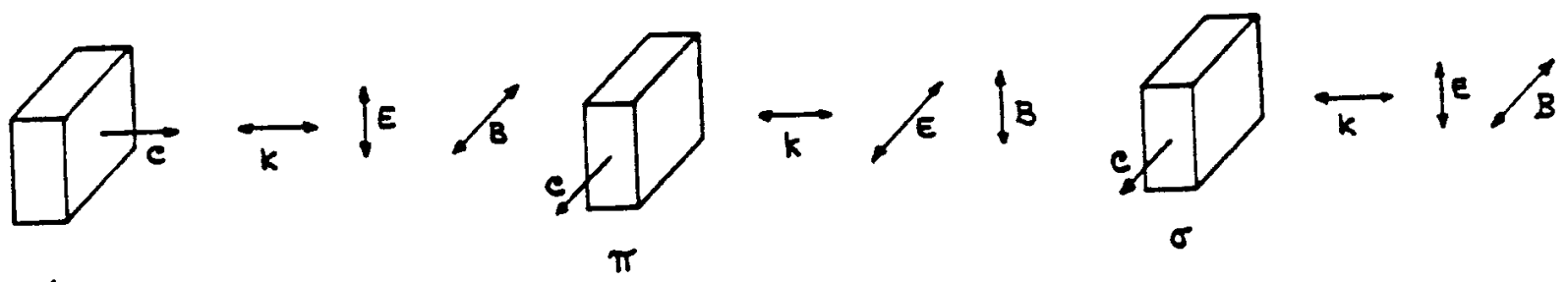

figura 1 - Tres possibilidades de incidencia para um cristal com um eixo de maior simetria.

Portanto tendo em mãos estes tres espectros $\alpha, \pi$ e $\sigma$ podemos saber qual transiçăo foi excitada por campo eletrico. qual foi excitada por campo magnetico e conhecer a direção de maior projeçăo destes dipolos, ou seja. com este metodo identificamos a natureza das transições e suas respectivas polarizaçסes. Por exemplo. se uma linha aparece nos espectros $\sigma$ e $\propto$, a transição relativa e dipolar eletrica ou quadrupolar eletrica, pois e excitada por um campo eletrico $E$ na direção perpendicular a c (direçăo do dipolo). Uma distinçăo entre estas duas transiçöes. a principio, se iaz examinando suas diferentes polarizações , mas devido a traca intensidade das linhas relativas a transiçoes quadrupolares e dificil de se identificar com certeza.

\subsubsection{REGRAS DE SELEÇAO}

Quando um ion de terra-rara e colocado num cristal, as regras de seleçăo que văo reger suas transiçoes são fortemente determinadas pela simetria local do campo cristalino. As regras. de seleção do Ion livre podem ser usadas apenas para entender as intensidades das linhas de absorçao e emissão. Por exemplo, uma transição é permitida para o lon no crital. mas viola as regras 
de seleça do ion livre; esta transiçao provavelmente sera iraca, pois Ioi permitida por uma perturbação, no caso o campo gerado pelos ions vizinhos. A seguir temos as regras de seleçao para ion livre.

E1: $\quad \Delta J=0, \pm 1 \quad J=0 \rightarrow J=0$

$\Delta \mathrm{L}=0, \pm 1 \quad \mathrm{~L}=0 \rightarrow \mathrm{L}=0$ paridade inicialfparidade final

$\Delta S=0$

M1 : $\quad \Delta J=0, \pm 1 \quad J=0 \rightarrow J=0$

$\Delta L=0 . \pm 1 \quad L=0 \rightarrow L=0$ paridade inicial=paridade inal

$\Delta S=0$

E2: $\Delta \mathrm{J}=0 . \pm 1 \quad \mathrm{~J}=\overline{\mathrm{U}}+\mathrm{J}=0.1$

$\Delta I=0 . \pm 1, \pm 2 \quad L=0+L=0$ paridade inicial=paridade ínal

$\Delta S=0$

onde E1 săo as transiçôs dipolares eletricas. M1 sao as transições dipolares magneticas e E2 são as transiçŏes quadrupolares elétricas.

Podemos dividir em dois casos a situação em que a simetria local do campo cristalino determina totalmente as regras de seleção. Se o cristal tem centro de inversão as transiçôes dipolares eletricas (E1) dentro de uma mesma configuração eletronica săo proibidas, pois possuem a mesma paridade, entretanto se este centro de inversåo nao existir as transiçðes ocorrem e as regras de seleção serao totalmente ditadas pela simetria. Analogamente, se as transiçoes forem entre configuraçðes. de paridades diferentes e o cristal tiver centro de inversão , as transiçסes dipolares magneticas (M1) e quadrupolares eletricas (E2) săo proibidas, e se a simetria nå tem centro de inversåo as regras de seleçao para E2 e M1 săo totalmente determinadas pelo grupo da simetria local do fon no cristal.

A probabilidade de transição por unidade de tempo na presença de uma perturbaçá é dada por : 


$$
W=\frac{2 \pi}{\hbar} \rho(k) \text { i } H^{\prime} \mathrm{rm}:=
$$

onde $\rho(k)$ e a densidade ininal de estados e $H^{\prime}$ rim $e$ o elemento de matriz do Hamiltoniano perturbativo. o qual conecta um estado inicial $m$ com um estado final $k$. Para transiçoes dipolares elétricas $H^{\prime}=e r . E \approx e$ P.A/C . Como $E$ e determinado externamente pela radiaçåo que induz a transiçăo, a probabilidade de transiçao nestas condiçðes e proporcional a $i r_{\mathrm{km}}{ }^{2}$ : ou $: \mathrm{p}_{\mathrm{km}}{ }^{2}:$. De forma similar, para transiços dipolares magneticas $W \approx i \mu_{m m}:^{2}=B^{2}:(2 S+L) i^{z}$, onde $\mu$ e operador momento dipolar magnetico e $B$ o magneton de Bohr.

o operador momento dipolar eletrico é um vetor polar. Isto significa que suas componentes se transformam como as coordenadas $x, y, z$. Portanto observando as funçðes de base do grupo de simetria em questao, sabemos qual e a representaçăo irredutivel de acordo com a qual este operador se transiorma. Tendo em mãos esta informaçăo podemos conhecer quais transiçres tèm elemento de matriz H'km diferente de zero, ou seja, quais transiçoses são permitidas via excitação dipolar eletrica, pois

ou

$$
\begin{aligned}
& \Gamma_{b E} \times \Gamma_{1}-\Gamma_{f} \\
& \Gamma+\times \Gamma_{D E} \times \Gamma_{1}-1-\Gamma_{1}
\end{aligned}
$$

onde $\Gamma_{D E} e$ a representaça irredutivel do grupo que se transforma como operador momento dipolar eletrico, $\Gamma_{\perp}$ é a representaçåo irredutivel do estado inicial e $\Gamma_{f}$ e a representação irredutivel do estado final.

De forma analoga conhecemos as transiçoes dipolares magneticas. Sabemos que o operador momento dipolar magnetico e um vetor axial, ou pseudo-vetor. Isto significa que ele se transforma como um vetor polar sob rotações mas e invariante sob inversão - Desta forma o conjunto de funçðes que val descrever este operador é $R_{x}$. $R_{r}, R_{z}$. 
Na tabela 1 temos a identificaçăo dos operadoes com a polarizaçăo dos espectros.

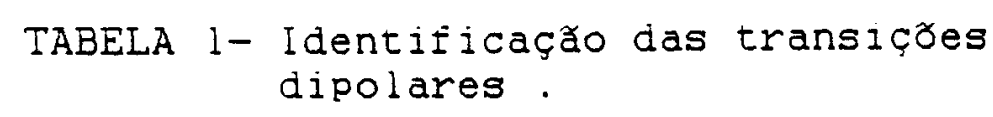

POL. OBSERVADA

CARACTER

\begin{tabular}{cc}
$\pi$ & $z$ \\
$\sigma$ & $R_{z}$ \\
$\pi, \propto$ & $\left(R_{x}, R_{Y}\right)$ \\
$\sigma, \propto$ & $(x, y)$ \\
\hline
\end{tabular}

Alem destas regras de seleção, para cristais que tenham centro de inversão a paridade tambem e um bom numero quàntico. pois se temos um operador impar, como e o caso do momento dipolar eletrico, ele so val conectar estados de paridades diferentes.

$$
\begin{aligned}
& O_{I}: \varphi_{F}>-\mid \varphi_{I}> \\
& o_{I}: \varphi_{I}>-: \varphi_{F}>
\end{aligned}
$$

No caso de um operador par. como e o caso do momento dipolar magnetico. ele so vai conectar estados de mesma paridade.

$$
\begin{aligned}
& \alpha_{\infty}\left|\varphi_{p}\right\rangle-\left|\varphi_{p}\right\rangle \\
& a\left|\varphi_{x}\right\rangle-\left|\varphi_{x}^{\prime}\right\rangle
\end{aligned}
$$

\subsubsection{INTENSIDADE DAS TRANSIC\&ES}

Atraves da teoria de grupos tambem podemos tirar algumas informaçós sobre a intensidade relativa das linhas. O metodo e simples, basta observarmos como se comportam as transiçóes na familia de simetrias que antecede a simetria desejada, indo no 
sentido das simetrias mais altas. Por exemplo, se uma transição é proibida em an e permitida em $C_{3}$, ela serámenos intensa do que as permitidas já em $a_{n}$, pois no primeiro caso a transiçăo passa a ser permitida por uma distorção do campo, ou seja. uma perturbação de segunda ordem.

\subsection{ESPECTRO DE ABSORCAO E EMISSAO DE TERRAS RARAS EM CRISTAIS}

\subsubsection{COEFICIENTES DE EINSTEIN PARA TERRAS-RARAS EM CRISTAIS}

A probabilidade de emissáo espontanea de um fóton de freguência w e dada por :

$$
A=1=\frac{8 \pi \omega^{5}}{3 h c^{2}}: M:=
$$

onde $\mathrm{Mi}$ é o elemento de matriz do operador dipolar elétrico ou magnetico. O Indice indica que a emissão foi relativa a transiçăo do nivel superior 2 para o nivel inferior 1.

Esta formula pode ser transformada se estivermos tratando de Ions terras-raras em cristais devido a dois efeitos :

1. Para transições dipolares eletricas a probabilidade de transiçăo é proporcional ao quadrado do elemento de matriz do momento dipolar eletrico e portanto ao quadrado do campo elétrico no local do ion. A expressão (15) deve ser multiplicada pelo fator $\left(E_{E F F} / E\right)=$, onde EEFF é o campo elétrico no ion e E o campo eletrico no vacuo.

2. A probabilidade de transiçăo e proporcional a densidade final de estados $k^{2} d k$, onde $k$ e vetor de propagaça dos fótons no cristal. Como

$$
k=\frac{w}{v}=\frac{w}{c / n}=\frac{n w}{c} \cdot
$$

com $n$ o indice de refraçăo, um fator extra $n^{3}$ tem que ser incluido na expresså para A. A razåo EfF/E pode ser expressa 
por :

$$
\underset{E}{E_{E E E}}=\underset{E_{E}}{E_{f i E E}} \frac{E_{C}}{E}
$$

onde $E_{c}=$ o valor medio do campo eletrico no cristal. E e $E_{c}$ devem corresponder a mesma densidade de fótons. Se indicarmos a constante dieletrica por $E$.

$$
\frac{E^{2}}{4 \pi}=\frac{E E_{x}}{4 \pi}
$$

ou

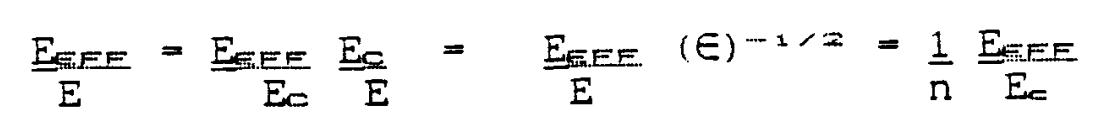

onde usamos a relação $\epsilon=\mathrm{n}^{x}$.

A expressão para a probabilidade de emissão espontanea. no caso de uma transiçăo dipolar eletrica, pode agora ser escrita

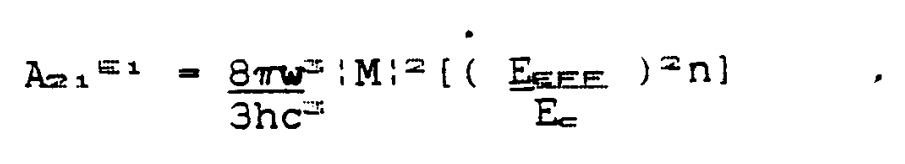

e para o caso dipolar magnético.

$$
A=1=\frac{8 \pi w^{m}}{3 h c^{3}}: M:^{2} n^{3}
$$

Podemos agora introduzir uma quantidade adimensional

$$
f_{12}=\frac{2 m w^{2}}{3 h e^{2}}: M i^{2}
$$

Esta quantidade e chamada força de oscilador da transiçăo . A probabilidade de emissão espontanea pode ser expressada em termos da força de oscilador

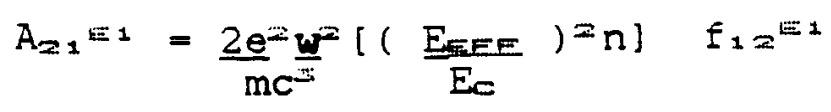

$$
\begin{aligned}
& A=1^{m 1}=\frac{2 e^{2} w^{2}}{m c^{2}} n^{\equiv} \quad f_{1 z^{m 1}}
\end{aligned}
$$

Um modo diferente de escrever a relação (23) é expressando $\mathrm{A}_{21}$ como $1 / \tau_{0}$ ou inverso do tempo de vida radiativo. No caso de 
dipolo elétrico temos

$$
f_{z 1}{ }^{E 1} \tau_{0}=\frac{m C^{2}}{2 e^{2} w^{2}}\left(E_{C} / E_{E F F}\right)=\frac{1}{n}=1.51\left(E_{C} / E_{E F F}\right)=\frac{\lambda_{0}{ }^{2}}{n}
$$

e no caso de dipolo magnetico

$$
f_{21}{ }^{m+1} \tau_{0}=\frac{m c^{2}}{2 e^{2} \omega^{2}} \quad \frac{1}{n^{m}}=1.51 \frac{\lambda_{0}}{n^{m}}
$$

onde $\lambda_{0}$ e o comprimento de onda da radiaçåo no vacuo.

Consideremos agora o coeficiente B de Einstein. A razãon." $\mathrm{A}=1 / \mathrm{B}=1$ e dada por

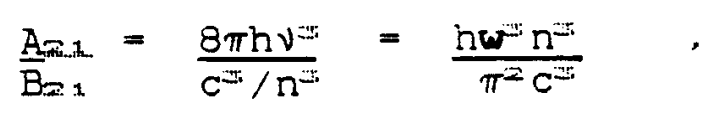

portanto $B \geq 1$ é dado por

$$
\begin{aligned}
& \mathrm{B}_{21}=1=\frac{\pi^{2} c^{3}}{h w^{-2} n^{2}} \quad A_{21}=1=\frac{8 \pi^{3}}{3 h^{2}} \quad i M_{1}=\left[\left(E_{C} / E_{E F F}\right)^{2} \frac{1}{n^{2}}\right] \\
& B=1^{M 1}=\frac{8 \pi^{3}}{3 h^{2}} \quad|M|^{x} \\
& \text { O coeficiente } B_{1}=\text { está relacionado com } B_{x i} \text { atraves de } \\
& \frac{B_{12}}{B_{21}}=\frac{g_{2}}{g_{1}}
\end{aligned}
$$

onde $g_{1}$ e $g_{2}$ săo os pesos estatisticos dos estados 1 e 2 respectivamente.

A presença do Indice de reiraçăo nas expressðes de $A$ e $B$ implica que as propriedades do meio afetam a intensidade da radiação .

Dexter ${ }^{14}$ sugeriu que EEFF seja dado por

$$
E_{E F F}=E_{\infty,}[1+(4 / 3) \pi \propto]=E_{0}\left(\frac{n^{2}+2}{3}\right)
$$

onde $\alpha$ é a polarizabilidade eletronica dos atomos. A expressão 
so é valida para lons de terras-raras em cristais ${ }^{ \pm 0}$.

\subsubsection{ESPECTRO DE ABSORCAO}

No lon livre as transições entre niveis de uma mesma configuraçao eletrônica podem ser apenas dipolares magneticas e quadrupolares elétricas, pois a paridade dos estados inicial e final é a mesma. Por outro lado, em cristais os diferentes termos da Hamiltoniana que representam a influencia do campo cristalino podem ter diferentes simetrias de inversăo; alguns termos pares e outros impares. Esta Hamiltoniana não comuta com o operador paridade, e deve conectar os estados do sistema não perturbado que tenham paridades diferentes, no caso mistura de fn e fn-1d. Quando tal mistura ocorre, transiçбes dipolares elétricas são permitidas.

Misturas de estados de diferentes paridades é tambem produzido por vibrações impares da rêde que são acopladas com 0 sistema iônico. O efeito desta mistura é a realização de certas transiçoes dipolares elétricas vibracionalmente induzidas . o numero $f$, ou força de oscilador destas transições, as quais sao eletrônicamente proibidas mas såo permitidas atraves da interaçå ion-rêde, são dependentes da temperatura.

- alargamento térmico de linhas de absorção é devido a transferência de energia do sistema iônico para o banho de fonons, o qual é acoplado simultaneamente pela transiçăo eletrônica. Como para os Ions de terras-raras o acoplamento com a rêde é pequeno o alargamento de suas linhas de absorça pela temperatura é insignificante.

Devido ao efeito da blindagem da camada $4 f$, o espectro de absorçăo de um determinado ion de terra-rara varia muito pouco de um cristal para outro. O número de linhas pode ser diferente 
mas o deslocamento de energia em relaçăo a energia do ion livre e pequeno, a largura das linhas e bem estreita e a força de oscilador e da ordem de $10^{-\infty}-10^{-7}$.

\subsubsection{ESPECTRO DE EMISSAO}

\subsubsection{INTRODUÇO}

Quando uma impureza e colocada num cristal ela passa a Iazer parte da rede e vibrar de acordo com seus modos normais de vibraçao. Esta interaça do 10 com a rede acaba por infiluenciar as caracteristicas dos espectros de absorçăo e emissåo.

Como se pode ver pela figura 2 , a interação do 10 n com a rède acarreta o aparecimento de um numero de estados para os quais 0 Ion pode transicionar com energias proximas a do estado eletrónico e isto alarga as linhas de absorção e emissão . A largura de linha, portanto, passa a ter dependencia com a temperatura. Para baixas temperturas as linhas se estreitam pois os modos normais de vibraçăo de maior energia vao ficando despopulados.

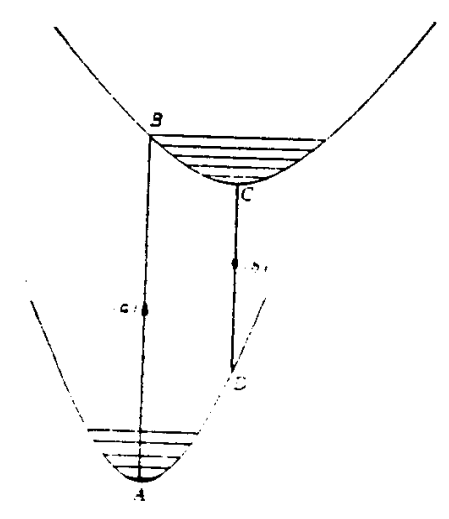

figura 2 - Absorçao e fluorescencia num sistema com deslocamento de Stokes : (a) absorçă, (b) ṫluorescencia.

Outro efeito que se observa atraves da figura 2 é que a energia da emissá é menor do que a da absorçá devido a perda 
de energia atraves de transferencia de energia para os fônons da rêde, ou seja, decaimento năo radiativo. Esta diferença entre a energia da emissão com a da absorçáo é chamado de "deslocamento de Stokes".

Para os Ions de terras-raras em cristais o "deslocamento de Stokes " "em geral, nao e significativo. Isto se deve ao rato dos elétrons que efetuam as transições interagirem musto iracamente com a réde, devido a blindagem das camadas $5 p$ e $5 s$. Neste caso. as curvas que surgem na regiao dos niveis de energia da configuração $4 f$ da impureza relativas a parte vibracional do cristal săo praticamente paralelas. Esta configuraçăo tambem explica a estreita largura de linha dos espectros de absorçå e emissão observadas para os terras-raras.

Na figura 3 temos um diagrama de niveis de energia usado para representar a fluorescencia de ions dentro de uma matriz cristalina. A luz incidente excita os fons do estado fundamental 1 para para um estado excitado representado pela banda de absorçåo 3 . Os ions entao decaem rapidamente atraves de uma transiçăo năo radiativa para o estado metaestavel 2 . Deste nivel fluorescente os lons decaem para o fundamental por um processo puramente radiativo e por outros processos que podem ser chamados de secundarios. A probabilidade por unidade de tempo que o ion passa por uma transiça do estado fundamental para a banda de absorçăo e dada por W. A probabilidade por unidade de tempo do nivel 2 para $\circ 1$ e $p_{z 1}$. Por simplicidade suporemos que para um ion no nivel 3 a probabilidade de decair para o fundamental é muito pequena em relaçăo a probabilidade de uma transiça radiativa para o nivel 2 . 


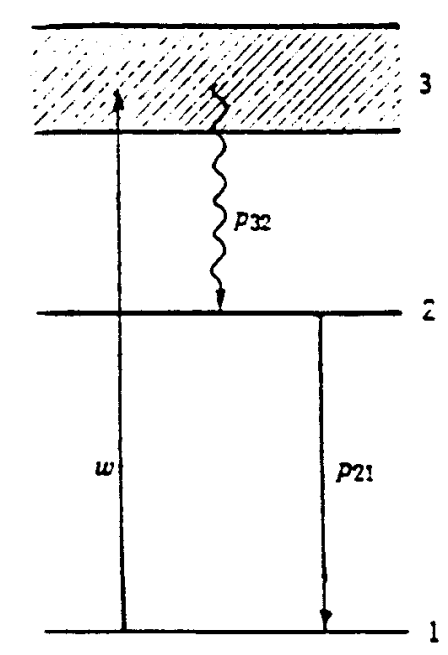

figura 3 - Sistema de tres niveis com um nivei fluorescente e
processos de decaimento rapicos encre os niveis 2 e 3.

Se No for o numero total de lons no sistema e $N_{1} \circ$ numero de Ions no estado iésimo, podemos escrever as seguintes equaçōes de taxa:

$$
\begin{aligned}
& \frac{d N_{3}}{d t}=W\left(N_{1}-N_{x}\right)-P \leq=N_{3} \\
& \frac{d N_{z}}{d t}=P=2 N_{z}-P_{x 1} N_{z} \\
& \frac{d N_{1}}{d t}=-\left(\frac{d N_{3}}{d t}+\frac{d N_{3}}{d t}\right)
\end{aligned}
$$

com

$$
\mathrm{N}_{1}+\mathrm{N}_{2}+\mathrm{N}=\mathrm{N}_{0}
$$

Em equilibrio, sob excitação contınua.

$$
\begin{aligned}
& 0=W\left(N_{1}-N\right)-P=2 E \\
& 0=P=N_{-3}-P_{21} N_{2}
\end{aligned}
$$

entao

$$
\left(w+p_{s=}\right) N_{s}=W N_{1}
$$

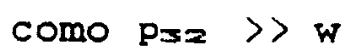

$$
P=2 N \approx W N_{1}
$$




$$
\mathrm{W}_{1}-\mathrm{P}_{21} \mathrm{~N}_{2}=0
$$

Considerando que $N \approx 0$ e tomanco a quarta equaçăo (32), podemos escrever :

$$
\text { W }\left(N_{0}-N_{2}\right)=P_{21} N_{2}
$$

ou

$$
\mathrm{Nz}=\frac{\mathrm{W} \mathrm{N}_{0}}{\mathrm{~W}+\mathrm{P}=1}
$$

Em (37) $N_{o}$ e $p=1$ såo constantes, entretanto $w$ e uma probabilidade proporcional a radiação incidente.

o tempo de vida do nivel 2 e o inverso de $\mathrm{p}_{21}$ e e chamado de tempo de vida da íluorescencia.

$$
\tau F=\frac{1}{P=1}
$$

Pode-se notar que a probabilidade $\mathrm{p}=1$ inclui todos os processos de decaimento que são originados do nivel 2 e terminam no nivel 1 . Estes processos săo decaimentos puramente radiativos, decaimentos vibracionalmente assistidos ou vibronicos, e os decaimentos nå radiativos.

\subsubsection{EFICIÊNCIA QUÂNTICA E O TEMPO DE VIDA DA FLUORESCÊNCIA}

Definimos eficiència quantica a seguinte quantidade :

$$
Y=\frac{n_{f}}{n_{m}}
$$

onde $\Omega_{f}=$ numero de quantas de luz emitidos por unidade de tempo na linha de zero fonon

$n_{-}=$numero de quantas de luz absorvidos por unidade de tempo.

Pode-se demonstrar que ${ }^{1=}$ 


$$
\mathrm{n}_{*}=\mathrm{n}_{-} \frac{\tau_{F}}{\tau_{0}}
$$

onde

$\tau_{0}^{-1}=\int w^{-1}\left(\lambda_{*}\right) d \lambda_{f}=$ tempo de $v i d a$ do estado fluorescente com war sendo a probabilidade de transicaro para apenas os processos radiativos.

De 40 e 41 temos que

$$
Y=\frac{\tau_{F}}{\tau_{0}}
$$

A eficiència quantica é então proporcional ao tempo de vida da fluorescencia.

o tempo de vida da fluorencencia e, em geral. menor do que o tempo de vida radiativo to devido a presença de outros processos de decaimento. Isto pode ser expressado da seguinte forma :

$$
\frac{1}{\tau_{F}}=\frac{1}{\tau_{0}}+\frac{1}{\tau_{\beta}}
$$

a qual indica que a probabilidade total de decaimento $\tau_{F^{-1}}$ é igual a soma das probabilidades do decaimento radiativo e dos processos competitivos.

\subsection{O CRISTAL LINBO=}

\subsubsection{A ESTRUTURA DO LINBO=}

Os monocristais de LiNbos exibem uma transição de fase estrutural a uma temperatura que varia de 1150 a $1210^{\circ} \mathrm{C}$ ( temperatura de Curie - $\mathrm{T}_{c}-$ ), dependendo da razăo Li/Nb. Em temperaturas abaixo de $T_{c}$, ou seja. na fase ferroelétrica do material. sua estrutura pertence ao grupo espacial romboedrico ( trigonal) R3c, e grupo pontual 3m (Csu). Acima de $T_{c}$ a 
estrutura passa para o grupo pontual apolar $3 \mathrm{~m}(\mathrm{D}=\mathrm{a})$.

No sistema trigonal duas células unitarias diferentes podem ser escolhidas: hexagonal e romboedrica. A celula unitaria hexagonal do LiNbos contem seis moléculas, enquanto a celula unitaria romboédrica convencional contém apenas duas. Por ser mais simples tomaremos para nossos estudos a célula hexagonal. $\mathrm{Na}$ figura 4 temos a célula unitária hexagonal do LiNbos : Nesta célula o eixo cristalino $c$ é definido como eixo de ordem 3 . Supoe-se $O$ sentido $+c$ do eixo como sendo $O$ sentido do crescimento $+z$.

Na tabela 2 temos as posições de cada ion na rede : Ii , $\mathrm{Nb} e \mathrm{O}$, nas duas fases do LiNbos. As outras posiçסes dos lons na celula unitaria podem ser derivadas pela aplicação dos elementos de simetria e do operador da rede $(0,0,0 ; 1 / 3,2 / 3,2 / 3 ; 2 / 3.1 / 3.1 / 3)+$, valido para ambas estruturas $:$

Como mostra a figura $4 b$, a rêde básica é constituida por seis planos equidistantes com normal paralela a $c$ formados por camadas planares de oxigenio. Entre estes planos os cations ficam empilhados alternadamente sobre o eixo c. A mudança a nivel estrutural que ocorre em $T_{c}$ é exatamente em relaçăo a posição dos cations. Na fase paraeletrica os cations ficam ou nos planos de oxigènio ( $\mathrm{Li}$ ), ou num plano equidistante dos planos de oxigênio (Nb) , como mostra a figura $5 a$. Estas posiçסes fazem a estrutura ser centro simétrica e portanto apolar. Agora quando a temperatura é menor que $T_{c}$, ou seja, fase ferroelétrica, os cations se deslocam de acordo com a figura $5 b$. Este deslocamento faz com que o litio fique dentro de um octaedro distorcido deixando outro octaédro com uma vacancia ( $V i$ ). O empilhamento ordenado de lugares com simetria octaedrica no eixo c torna-se : $\ldots \mathrm{Nb} V i$ Li $\mathrm{Nb} \quad \mathrm{Vi}$ Li Nb Vi Li $\ldots$ 
O desiocamento destes lons é : 0.71 A para o litio e $0.26 \mathrm{~A}$ para o niobio.
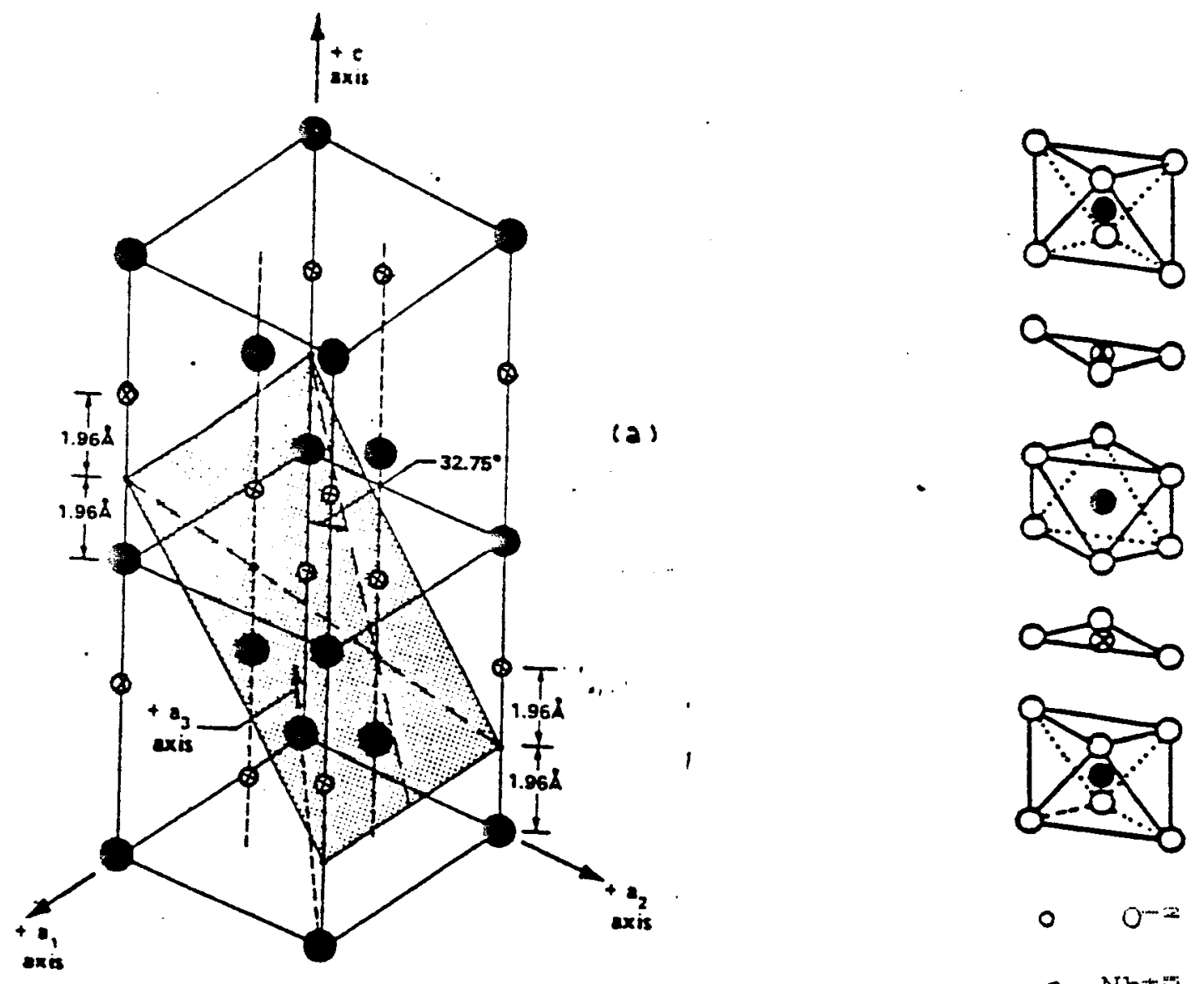

(b)
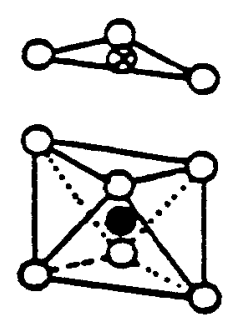

$0 \quad 0^{-2}$

- $\mathrm{Nb}^{+5}$

- $\mathrm{Li}^{+1}$

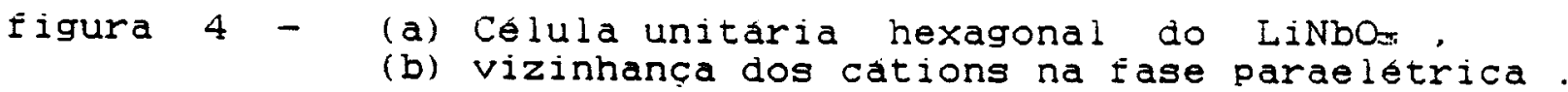
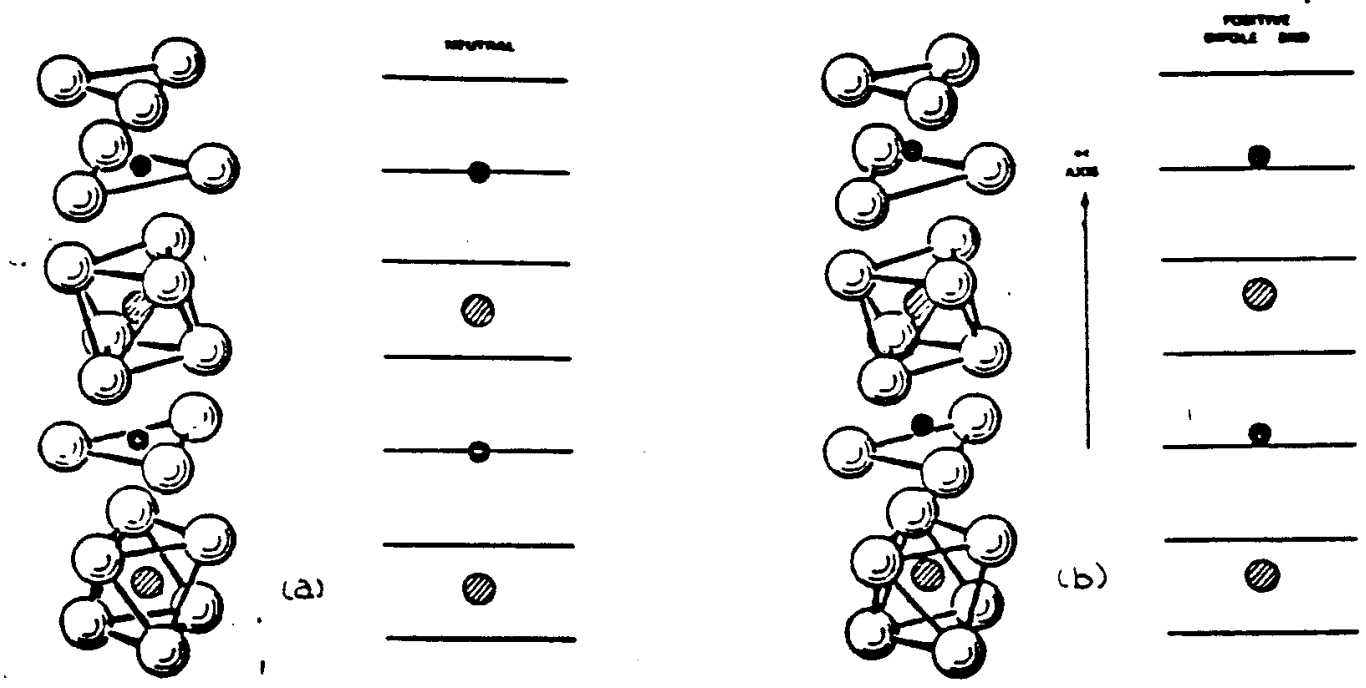

figura 5 - (a) Posiçбes dos lons na iase paraeletrica e

(b) Posiçres do lons na fase ferroeletrica do LiNbos. 
TABELA 2 - Posiçסes principais dos ions $\mathrm{Li}$, Nb e O na rede do LiNbO= $\left(a_{H}=5.148 \mathrm{~A}, \mathrm{C}_{H}=13,86 \mathrm{~A}\right)$ (de acordo com [37]).

Estas posiçסes deslocadas dos fons de litio e niobio mostradas na figura 5 (b) fazem com que o cristal exiba uma polarizaçăo espontânea. Assim $\circ$ LiNbos, abaixo de $T_{c}$, é considerado um cristal ferroelétrico de deslocamentolo.

O arranjo de oxigenios mostrado na figura $4 \mathrm{~b}$, é idealizado. Na verdade existe uma leve distorção. Como se pode ver na figura 6 os oxigenios nao formam colunas paralelas ao eixo trigonal . como seria no arranjo de empacotamento hexagonal compacto.
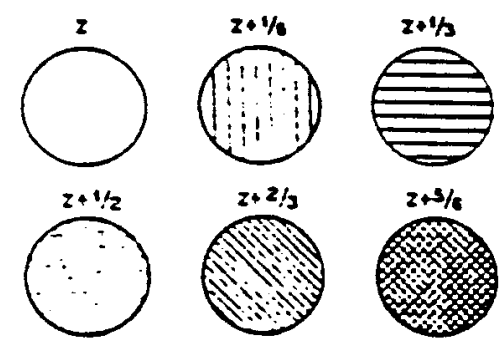

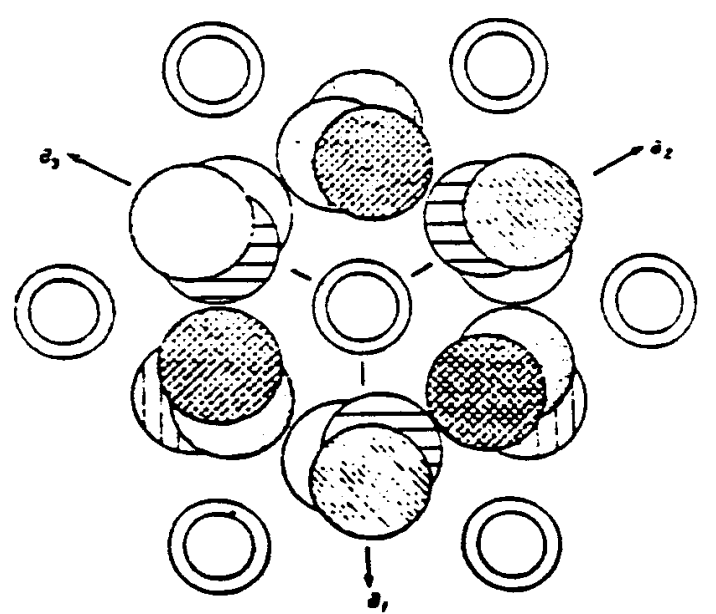

figura 6 - Distorça dos planos de oxigenio no LiNbos. 


\section{5 .2 O CRESCIMENTO DO LINBO=}

Os cristais foram crescidos pelo grupo de crescimento de cristais deste departamento . Maiores detalhes sobre o crescimento pode-se encontrar na referencia [12].

Os monocristais de LiNbos são crescidos pelo metodo de Czochralski, utilizando-se um sistema automatico de crescimento de cristais - AUTOX Crystal Growth System ( Cambridge Instruments, .

Num cadinho de platina e colocada uma composição congruente de $\mathrm{Li}=\mathrm{CO}=$ e $\mathrm{Nb} z \mathrm{O}=$ ( oticamente puros) obtida do diagrama de fase desses componentes, nas proporções de 48,6 mol\% de $\mathrm{Li}_{2} \mathrm{CO}_{3}$ e $51,4 \mathrm{~mol} \%$ de $\mathrm{Nb}=\mathrm{O}=$

A semente utilizada e orientada de maneira que o crescimento ocorra ao longo da direção [001], isto e c, pois apresenta uma maior estabilidade de crescimento e uma menor densidade de deslocações nos cristais.

Os monocristais de LiNbOs crescidos pelo metodo de Czochralski são obtidos na fiase paraeletrica, uma vez que a temperatura de transição de fase e $1210^{\circ} \mathrm{C}$ e a temperatura de fusao da composiçăo é de $1253^{\circ} \mathrm{C}$.

Este fato provoca, durante o processo de crescimento, o aparecimento de uma estrutura de dominios de dipolos ferroeletricos orientados ao acaso, que modifica as caracteristicas oticas, eletricas, acusticas, etc. do LiNbos, impossibilitando sua aplicaça tecnologica.

- metodo mais eficaz para a orientaça dos dominios ferroeletricos no LiNbos consiste na aplicação de um campo elétrico no cristal durante o processo de crescimento. 


\subsubsection{INCORPORACAO DE DOPANTES NA MATRIZ LINBO-}

0 niobato de litio pode ser impurificado com um numero razoavelmente alto de lons, e muitas tecnicas tais como medidas oticas, RPE, RMN, e medidas Móssbauer tem sido utilizadas para se conhecer os sitios que as impurezas ocupam, mas ate agora não se obteve nenhuma evidència $e$ todas as discussores são especulativas, faltando mais estudos ou outras tecnicas experimentais que permitam comprovar o modelo. A simetria local da maior parte das impurezas cationica e trigonal, como esperado. Há apenas alguns exemplos com ions de terras-raras onde a vizinhança parece mostrar uma distorção ortorrombica ou monoclinica. O sitio com simetria trigonal concorda com o sitio dos lons sobre o eixo c, como os Ions $\mathrm{Li} e \mathrm{Nb}$.

Segundo Nassau=z a incorporaçăo de impurezas e governada por quatro fatores :

1 - raio iônico

2 - ajuste de carga

3 - carater covalente das ligaçres

4 - numero de coordenação

Como os lons de niobio e lftio têm aproximadamente o mesmo raio iônico ( ver tabela 3 ), este fator pode ser desprezado na análise de substituiçăo. Os lons com valència +4 e +5 ( proximas a do niobio,, provavelmente substituirăo o niobio. o mesmo vale para os lons com valència +1 e +2 (proximas a do litio) que provavelmente substituirao o litio. O maior problema surge para lons que tenham valencia +3. como o erbio. Para estes casos espera-se que as impurezas sejam incorporadas em ambos os sitios da rede e talvez nas vacancias estruturais. 


\begin{tabular}{cc} 
TABELA 3 - Raio iônico do litio e nióbio \\
\hline raio iônico $(\AA)$ \\
$L^{+1}$ & 0,68 \\
$\mathrm{Nb}^{+\ldots+}$ & 0.69 \\
\hline
\end{tabular}

\subsubsection{ALGUMAS PROPRIEDADES ÓTICAS DO LINBO=}

\subsubsection{ESPECTRO ÓTICO}

O LiNbo muito puro e bem oxidado e um cristal transparente. No espectro ótico, a regiao de transparencia deste cristal e aproximadamente de $0.35 \mu \mathrm{m}\left(28500 \mathrm{~cm}^{-1}\right)$ a $5 \mu \mathrm{m}\left(2000 \mathrm{~cm}^{-1}\right)$, onde começa a banda dẹ absorçăo das vibraçós da rêde. O regime de vibraç̧es da rêde se estende até proximo de $80 \mu \mathrm{m}\left(125 \mathrm{~cm}^{-1}\right)$.

\subsubsection{BIRREFRINGÊNCIA}

- LiNbos e um cristal uniaxial, e portanto, suas propriedades sao caracterizadas por dois 1 ndices de refraçăo $n^{-}$ ( Indice de refraçăo extraordinario) e no ( Indice de refraçăo ordinario ) .

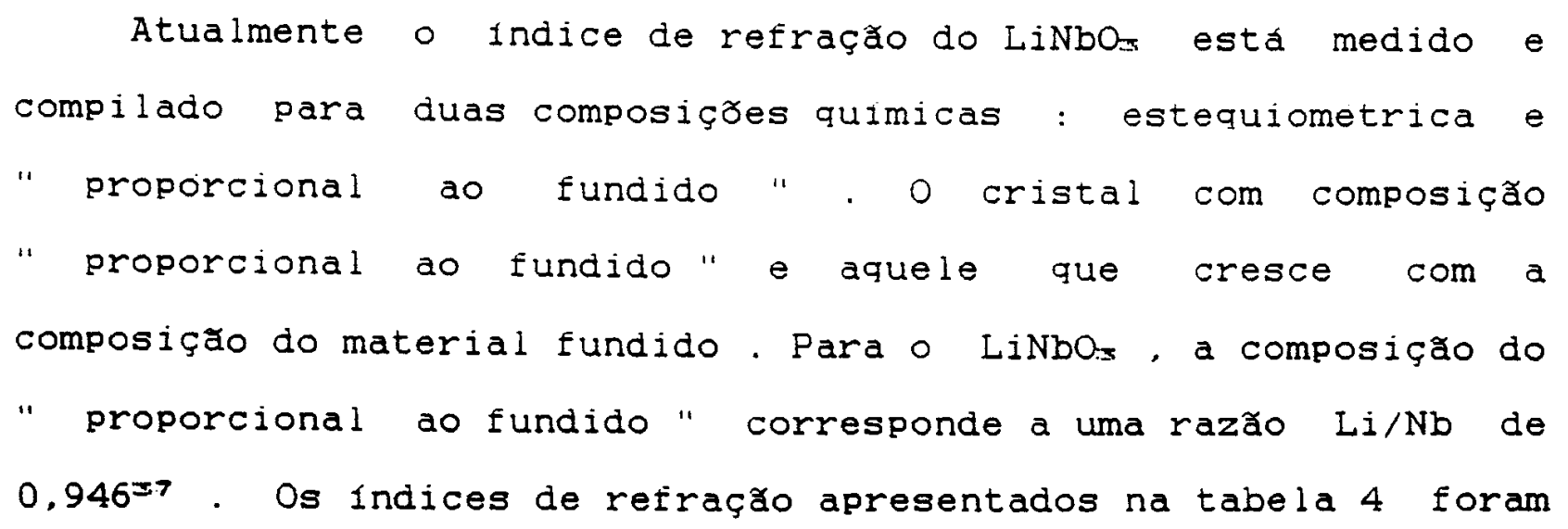


linearmente interpolados para comprimentos de onda laser mais comuns.

TABELA 4 - Indices de refraçao ordinario e extraordinarios7 do LiNbos congruente para $24,5^{\circ} \mathrm{C}$.

\begin{tabular}{|c|c|c|}
\hline$\lambda(\sin )$ & $n^{0}$ & $n^{e}$ \\
\hline 0.40463 & 2.4317 & 2.3260 \\
\hline 0.43584 & 2.3928 & 2.2932 \\
\hline 0.46782 & 2.3634 & 2.2683 \\
\hline 0.47999 & 2.3541 & 2.2605 \\
\hline 0.50858 & $2.3350^{\circ}$ & 2.2448 \\
\hline 0.54607 & 2.3165 & 2.285 \\
\hline 057696 & 2.3040 & 2.2178 \\
\hline $0 \leq 57897$ & 2.3032 & 2.2171 \\
\hline 0.58756 & 2.3002 & 2.2147 \\
\hline 0.64385 & 2.2835 & 2.2002 \\
\hline 0.66782 & - 2.2778 & $2.195 \equiv$ \\
\hline 0.70652 & 22699 & 2.1886 \\
\hline 0.80926 & 2.2541 & 2.1749 \\
\hline 0.87168 & 2.2471 & $2.168 \varepsilon$ \\
\hline 0.93564 & 2.2412 & 2.1639 \\
\hline 0.95998 & 2.2393 & $2.16=2$ \\
\hline 1.0140 & 2.2351 & 2.1584 \\
\hline 1.09214 & 2.2304 & 2.1545 \\
\hline 1.15392 & 2.2271 & 2.1517 \\
\hline 1.15794 & 2.2269 & 2.1515 \\
\hline 1.28770 & 2.2211 & 2.1464 \\
\hline 1.43997 & $2.215 i$ & 2.1413 \\
\hline 1.63821 & $2.208:$ & 2.1556 \\
\hline 1.91125 & 2.1954 & 2.1250 \\
\hline 218428 & 2.1912 & 2.1212 \\
\hline 239995 & $2.18: 0$ & 2.1151 \\
\hline 2.61504 & 2.1765 & 2.1087 \\
\hline 2.73035 & $2.172=$ & $2.105 j$ \\
\hline 2.89733 & 2.1657 & 2.0999 \\
\hline 3.05148 & 2.1594 & 2.0946 \\
\hline
\end{tabular}

Em 1966, Hobden e Warner mediram os 1ndices de reiraçaoso $\mathrm{n}^{-}$e $\mathrm{n}^{\circ}$ em temperaturas entre 19 e $374^{\circ} \mathrm{C}$ e para comprimentos de onda de 447 a $707 \mathrm{~nm}$. Para estes dados eles derivaram duas equaçðes tipo Sellmeier para $n^{-} e n^{\circ}$. onde $T$ e a temperatura em graus Kelvin e e o comprimento de onda em $\mathrm{nm}$ :

$$
\begin{gathered}
\left(n^{\circ}\right)=4.913+\frac{1.17310^{=}+1.6510^{-2} T^{2}}{\lambda^{2}-\left(2.1210^{2}+2.710^{-5} T^{2}\right)^{2}}-2.7810^{-9} \lambda^{2} \\
\left(n^{-}\right)^{2}=4.5567+2.60510^{-7} T^{2}+\frac{0.97010^{5}+2.7010^{-2} T^{2}}{\lambda^{2}-\left(2.0110^{2}+5.410^{-5} T^{2}\right.} \\
-2.2410^{-0} \lambda^{2}
\end{gathered}
$$


Estes resultados podem divergir dependendo da composiçåo do cristal. O indice de refraçao extraordinario pode mudar ate em $0.03^{\mp 7}$ dependendo da razăo $\mathrm{Li} / \mathrm{Nb}$ e $\circ$ indice de reiraçăo ordinario e independente da composição.

A birrefringencia de um material e definida como a diferença $\left(n^{*}-n^{\circ}\right)$. A birretringencia do LiNbos é aproximadamente $-0,08$. 0 sinal negativo pode ser entendido qualitativamente considerando o efeito dos dipolos atômicos que sao induzidos pela aplicaçåo de um campo elétrico. Quando o campo é aplicado os elétrons carregados negativamente e o nucleo carregado positivamente separam-se e produzem um dipolo eletrico. Se o dipolo induzido produz uma diminuiçăo do campo eletrico nos seus atomos vizinhos, a susceptibilidade $X$-decresce. Do mesmo modo, se os dipolos induzidos produzem um aumento no campo de seus atomos vizinhos, a susceptibilidade dieletrica e a permissividade relativa soirem um acrescimo

$$
\epsilon / \epsilon_{0}=1+X
$$

Os efeitos de polarizaçăo e despolarizaçăo dos dipolos de oxigenio induzidos sobre os atomos vizinhos mais proximos produzem uma contribuiçå dominante para a susceptibilidade dieletrica do $\mathrm{LiNbO}=$. Se aplicarmos um campo eletrico paralelo ao eixo ótico, os dipolos de oxigenio induzidos tem um efeito de despolarizaçăo sobre os oxigenio vizinhos. Mas se aplicarmos um campo eletrico perpendicular ao eixo otico, os dipolos de oxigenio induzidos tem um eieito de polarizaçao sobre os oxigenios vizinhos. Portanto $\chi$ - paralelo do eixo otico e menor que $\circ \chi$ - perpendicular ao eixo otico. Assim. o Indice de refração extraordinario principal do $\mathrm{LiNbO}_{3}\left(\mathrm{n}_{-}=\left(\epsilon_{3} / \epsilon_{0}\right)^{1 / 2}\right)$ é menor do que 0 indice de refraçao ordinario $\left(n_{0}=\left(\epsilon_{11} / \epsilon_{0}\right)_{1 / 2}\right)$. 


\subsubsection{EFEITO FOTOREFRATIVO}

As primeiras observaçōes do efeito fotorefrativo (variaçăo do Indice de refração induzida oticamente) em cristais de LiNbOs foram feitas por Ashkin e outros, a aproximadamente vinte anos , durante uma experiência de geraçăo de segundo harmónico ${ }^{32}$. Eles observaram que quando um feixe de laser intenso incidia sobre o cristal, causava uma variaçăo local do indice de refraçăo. Esta variaçăo do indice de reiraçăo distorcia a frente de onda do feixe transmitido, e por isto o efeito foi inicialmente chamado de "dano otico" , desde que impedia a utilizaçăo destes cristais em aplicaçôs de ótica năo linear.

Apesar de ser um problema para aplicações de ótica nao linear, Chen mostrou no entanto, que uma possivel aplicaçå deste efeito seria a obtençăo de memorias oticas, logo era importante conhecê-10:a.

O modelo teorico de Chen para descrever o efeito fotoretrativo no LiNbos e basicamente o seguinte : se incidirmos luz laser normal do eixo $c(z)$ do cristal e em uma pequena area. eletrons sao fotoexcitados para fora da regiao iluminada e posteriormente capturados por armadilhas proximas a periferia do feixe de luz. Forma-se entao um campo elétrico interno local entre os eletrons que foram capturados e os centros positivos ionizados na regiăo iluminada. O campo de cargas $E_{1}$ da origem então a variaçao do indice de refraçao $n_{n}$, via efeito eletrootico intrinsico do material.

Quando a luz e removida, o efeito tende a permanecer por semanas ou meses, a menos que se aqueça o cristal a uma temperatura de aproximadamente $200^{\circ} \mathrm{C}$ ou o cristal seja iluminado por inteiro por uma luz homogenea e intensa que redistribui os eletrons novamente por todo o cristal. 
A tentativa de descobrir a origem destes elétrons, bem como os mecanismos de transporte envolvidos, levou Glass a descoberta de um novo efeito conhecido como efeito fotovoltáco. Segundo Glass, quando os cristais de LiNbOs sao iluminados, surge na direçåo c (z) uma fotovoltagem e uma fotocorrente estacionaria mesmo sem a aplicação de campos elétricos externos. Esta fotocorrente flui da face $+c$ para $-c$ (elétrons de $-c$ para $+c$ ) e pode ser medida através de um eletrometro=2

Glass tambem concluiu que o efeito fotovoltaico depende essencialmente da presença de impurezas como os metais de transição tipo ferro. rhodio, cobre, etc; dos quais o ferro e - rhodio são os mais eficientes. A descriçăo de Glass para o efeito fotorefrativo é a seguinte: Quando o cristal é exposto a luz, eletrons são excitados do 1 on $\mathrm{Fe}^{+2}$ para a banda de condução do cristal, sendo posteriormente capturado por uma armadilha, o Ion $\mathrm{Fe}^{+x}$.

\subsubsection{EFEITOS DE IMPUREZAS NAS PROPRIEDADES DO LINBO=}

\subsubsection{IMPUREZAS NO LiNbOs}

Os primeiros cristais que foram crescidos a vinte anos atras tinham uma cor marron no seu estado como crescido. Eles podiam ser clareados atraves de um demorado tratamento termico em atmosfera de oxigenio. Esta coloração era certamente devido a impurezas . Atualmente os cristais crescidos "puros" sao bem transparentes.

A impurificaça do linbos é facil porque a rede é capaz de se ajustar para um grande número de adiçzes. Ions de metais de transiçăo podem ser incorporados até atingir uma concentraça acima de alguns moles e terras-raras em torno de 16. 
Os metais de transição sao conhecidos por sua forte influencia na absorção otica e no ejeito fotorerrativo. Entretanto. suas concentraçðes podem ser controladas na medida do possivel no material inicial, antes do crescimento.

Além dos metais de transiçăo e dos terras-raras hà ainda outras impurezas que as vezes podem ser encontradas no niobato de litio tais como: Mo, $W$, os halogeneos, Al, os ions alcalinos, $\mathrm{Si}$, $\mathrm{B}$ e C . Algumas destas impurezas têm influència nas propriedades eletricas do LiNbos, especialmente sobre a condutividade iônica.

Tambem foi observado a incorporaçăo de hidrogènio em $\mathrm{LiNbO}_{3}$ e esta impureza pode ser ou não conveniente, dependendo da aplicação desejada. A seguir mostramos algumas propriedades do LiNbO= com hidrogênio.

\subsubsection{PROPRIEDADES DO LINBO= DOPADO COM FERRO}

- cristal de LiNbos dopado com ferro numa concentraçăo de aproximadamente $1 \%$ apresenta uma coloraçăo entre o vermelho e o marrom . Nesta matriz o ferro pode assumir duas valências $+2 e$ +3 .

Seu espectro de absorção apresenta uma banda em $485 \mathrm{~nm}$ que e relativa a interaçăo entre $\mathrm{Fe}^{+2}$ e $\mathrm{Nb}^{+=}$e seu espectro de $\mathrm{RPE}$ apresenta linhas caracteristicas do $\mathrm{Fe}^{+ \pm}$.

A importancia de se ter o controle da concentração dos lons $\mathrm{Fe}^{+=}$e $\mathrm{Fe}^{+-}$vem do tato deles serem os principais responsaveis pelo efeito fotorefrativo e dependendo da aplicaça desejada pode-se querer mais ou menos ierro na amostra. Por exemplo. quem deseja utilizar o LiNbos para holografia necessita de altas concentraçסes de ferro; entretanto. quem quer usar o LiNbos como cristal laser deseja que o cristal possua as menores 
concentraçoes possiveis.

\subsubsection{PROPRIEDADES DO LINBO $=$ DOPADO COM OH-}

Estes átomos de hidrogênio eståo sempre associados com lons de oxigênio para formar grupos $\mathrm{OH}^{-}$. A incorporaçăo de $10 n s \mathrm{OH}^{-}$ gera uma banda de absorçăo caracteristica na regiåo do infravermelho proximo em torno de $2,87 \mu \mathrm{m}$. Esta banda e completamente polarizada perpendicularmente do eixo c e é composta por pelo menos duas subandas que nao sao completamente resolvidas mesmo em baixa temperatura.

2.60 ION Er+3

\subsubsection{CARACTERISTICAS GERAIS DOS ÍONS DE TERRAS-RARAS}

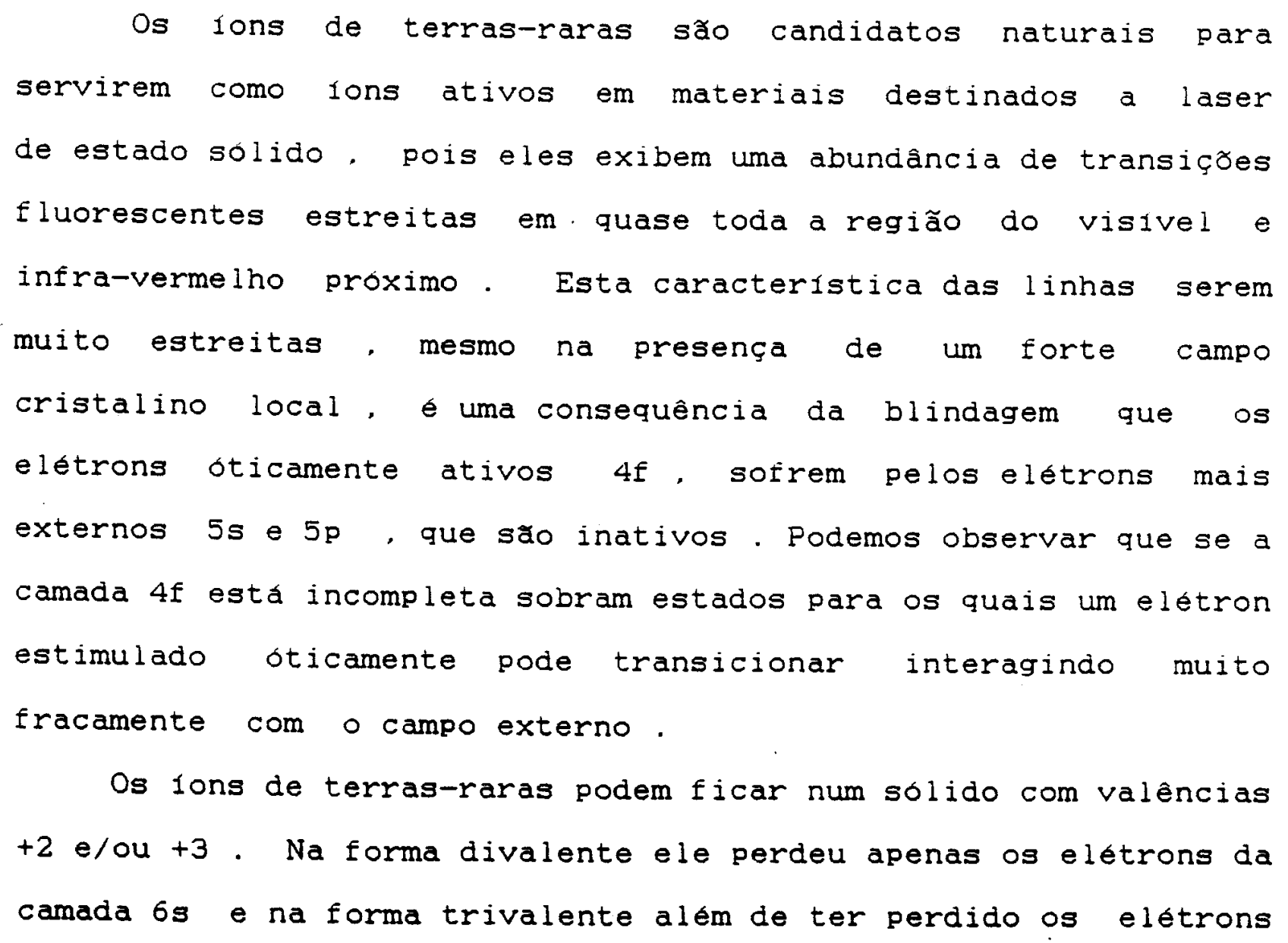


de $6 s$, ele pode ter perdido eletrons de $5 d$, se tiver elétrons $5 d$, ou ter perdido de $4 f$. Na tabela 5 temos o preenchimento das camadas dos terras rara.

TABELA 5 - Estrutura eletronica dos elementos 59 a 71.

Numero Elemento eletrons dá camada

meis superior

\begin{tabular}{|c|c|c|c|c|c|c|c|}
\hline 54 & Xenon. Xe & $4 d^{4} 0$ & - & $5 s^{2}$ & $5 p^{\prime \prime}$ & - & - \\
\hline 59 & Praseodymium. Pr & $4 d^{\prime 0}$ & $4 / 3$ & $5 s^{2}$ & $5 p^{8}$ & - & $6 s^{2}$ \\
\hline 60 & Neodymium. No & $4 \sigma^{10}$ & $4 / 4$ & $5 s^{2}$ & $5 p^{6}$ & - & $6 s^{2}$ \\
\hline 61 & Promelhium. Pm & $4 d^{10}$ & $4 f^{5}$ & $5 s^{2}$ & $5 n^{n}$ & - & $6 s^{2}$ \\
\hline 62 & Samarium. Sm & $4 d^{\circ}{ }^{\circ}$ & $4 f^{\circ}$ & $5 s^{2}$ & $50^{6}$ & - & $6 s^{2}$ \\
\hline 63 & Europlum. Eu & $4 d^{\prime 0}$ & $4 \Gamma$ & $5 s^{2}$ & $50^{*}$ & - & $6 s^{2}$ \\
\hline 64 & Gadolinium. Gd & $4 d^{20}$ & $4 f^{\mathrm{B}}$ & $5 s^{2}$ & $5 p^{6}$ & $5 d$ & $6 s^{2}$ \\
\hline 65 & Terbium, To & $4 d^{10}$ & $4 f^{\prime \prime}$ & $5 s^{2}$ & $5 n^{n}$ & - & $6 s^{2}$ \\
\hline 66 & Dysprosium. Dy & $4 d^{10}$ & $4 f^{\prime \circ}$ & $5 s^{2}$ & $5 p^{6}$ & - & $6 s^{2}$ \\
\hline 67 & Hoimium, Ho & $4 d^{\circ} \circ$ & $4 f^{\cdots}$ & $5 s^{2}$ & $5 p^{6}$ & - & $6 s^{2}$ \\
\hline 68 & Erbium. El & $4 d^{\circ}$ & $4 f^{\prime 2}$ & $5 s^{2}$ & $5 n^{6}$ & - & $6 s^{2}$ \\
\hline 69 & Thulium. Tm & $4 d^{n}$ & $4 r^{3}$ & $5 s^{2}$ & $5 n^{n}$ & - & $6 s^{2}=$ \\
\hline 70 & Ytterbium, Yb & $4 d^{10}$ & $4 f^{14}$ & $5 s^{2}$ & $50^{6}$ & - & $6 s^{2}$ \\
\hline 79 & Lutetium. Lu & $4 d^{\circ}{ }^{\circ}$ & $4 r a$ & $5 s^{2}$ & $5 p^{\kappa}$ & $5 d$ & $6 s^{2}$ \\
\hline
\end{tabular}

\subsubsection{CARACTERISTICAS DO ERBIO}

- érbio é o elemento de numero atómico 08 na tabela

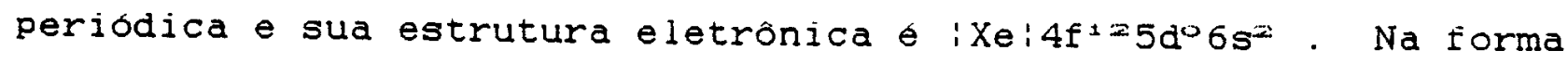
trivalente sua estrutura eletrônica passa a ser $4 f^{11}$ e os niveis de energia dentro desta configuraça estao na tabela 6 . 
TABELA 6 - Niveis de energia do Er- livre e suas respectivas energias.

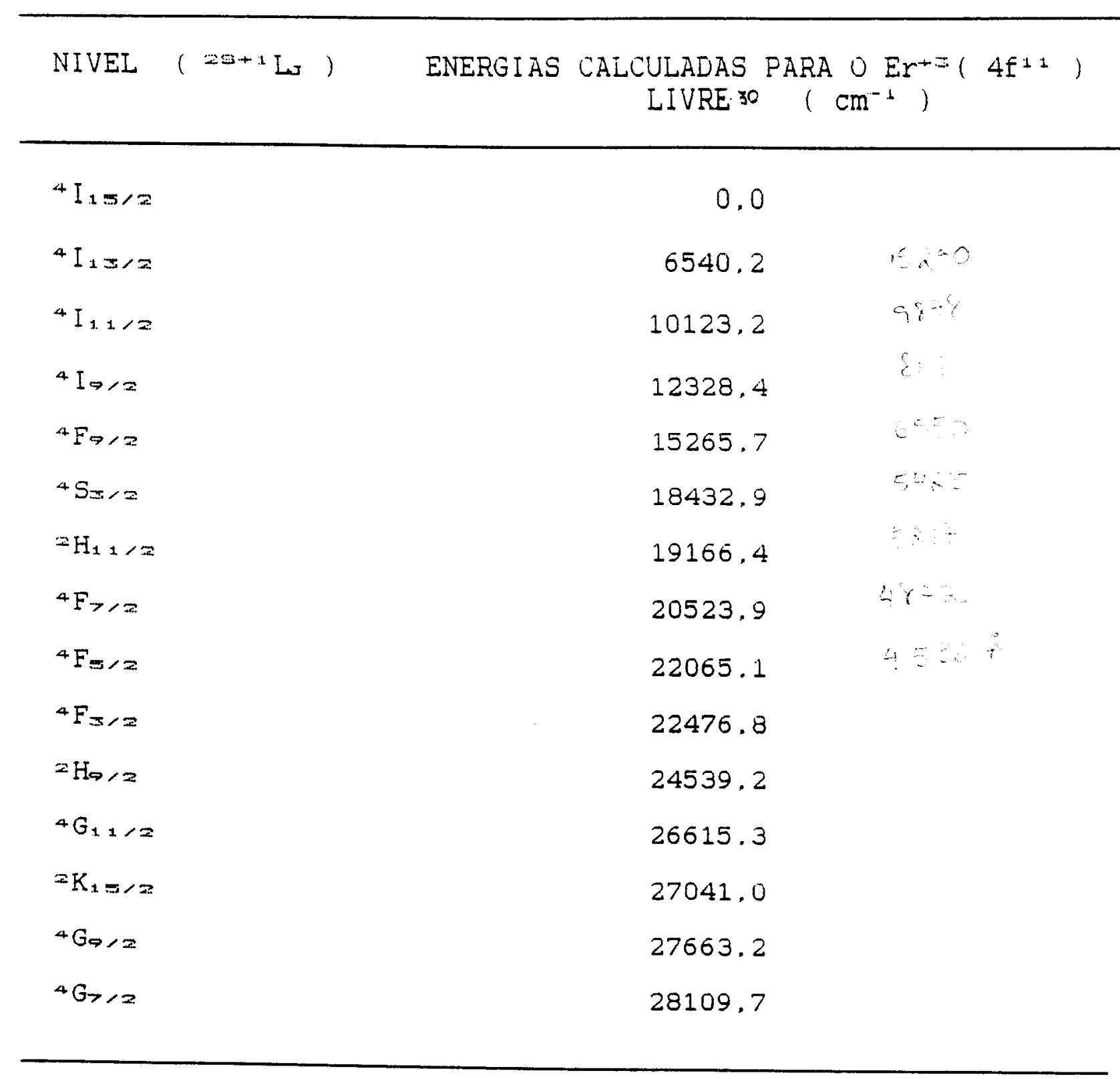

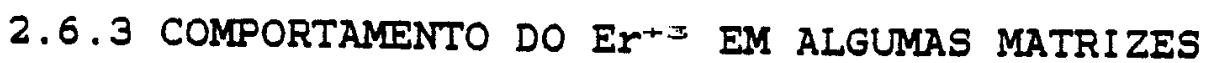

Muitos estudos têm sido feitos sobre as propriedades de absorçăo e luminescencia do erbio trivalente em varias matrizes para determinar seu potencial como ion laser. As oscilaçбes laser mais frequentemente observadas estao na regiao de 1.53 a $1.66 \mu \mathrm{m}$ que corresponde a transiçăo ${ }^{4} \mathrm{I}_{13 / 2} \rightarrow{ }^{4} \mathrm{I}_{15 / 2}$, onde ${ }^{4} I_{15 / 2}$ é o estado fundamental. Emissao estimulada em torno de $1.6 \mu \mathrm{m}$ e interessante pois o olho émenos sujeito a danos na 
retina nesta faixa do espectro, devido a, fortemente reduzida transmissividade do meio ocular neste comprimento de onda e tambem por ser muito útil para comunicaçoes por fibra ótica, pois em $1.5 \mu \mathrm{m}$ elas apresentam um minimo de perdas por transmissao .

As matrizes mais comumente dopadas com $\mathrm{Er}^{+\mathrm{i}}$ sao : YAG, YLF, YAlOs, $\mathrm{LaF}_{3}, \mathrm{CaWO}_{4}, \mathrm{CaF}_{2}$ e varios vidros. 0 nivel terminal do Er-3 está entre $525 \mathrm{~cm}^{-1}$ para Er:YAG e 50 $\mathrm{cm}^{-1}$ para Er:vidro. A temperatura ambiente todos os niveis do estado terminal ${ }^{4} \mathrm{I}_{15}=$ săo populados em algum grau. portanto esta transiçăo forma um sitema de très niveis com um alto "threshold". A açăo laser e geralmente realizada ou por diminuição da temperatura para despopulaçăo dos niveis mais altos do nivel ${ }^{4} \mathrm{I}_{1=/ 2}$, ou utilizando-se materiais dopados tambem com o Ion trivalente itérbio que melhora a eficiencia do bombeio otico via transferencia de energia Yb+x-Er+ $\mathbf{F}^{+}$. Os lasers de érbio em vidro que tem sido desenvolvidos usam materiais a base de fosfato e silicato e såo dopados com neodimio e itérbio em adiçăo ao érbio. O neodimio e o iterbio atuam como agente sensibilizador por absorver luz bombeada nas regioes onde o erbio e relativamente transparente.

\subsection{INTRODUÇAO TEÓRICA A TECNICA DE RESSONÂCIA PARAMAGNETICA} ELETRO̊NI CA

\section{7 .1 INTRODUÇAO}

Uma substancia paramagnetica pode ser definida como uma substancia que nao possui momento magnetico resultante na ausencia de um campo externo, mas adquire um momento magnético na direçå de um campo aplicado cuja intensidade é uma funçao do campo. Tal momento resultante é devido aos dipolos induzidos, 
os quais aparecem apenas atraves da açăo de um campo e é resultado da carga em movimento dos elétrons que constituem cada átomo ou ion. Em todos os casos isto leva a um momento de dipolo negativo ( isto é, um momento de dipolo anti-paralelo ao campo aplicado, e portanto ao diamagnetismo que e uma propriedade básica para todas as substâncias paramagnéticas.

Uma classe importante de substancias paramagneticas são os materiais constituidos por atomos ou rons com momento magnetico permanente de magnitude nuclear ou atomica. Na ausència de um campo externo tais dipolos estão orientados aleatoriamente, mas a aplicação de um campo externo resulta na redistribuiçăo sobre as varias orientações de uma forma que a substancia adquire um momento magnetico. Tal dipolo magnetico permanente ocorre apenas quando o atomo ou o nucleo possui um momento angular resultante, e os dois sao relacionados pela formula

$$
\mu=\gamma_{G}
$$

onde $\mu$ émomento de dipólo magnetico, $G$ o momento angular 1 um multiplo inteiro ou semi-inteiro de $(h / 2 \pi)=\hbar$ onde $h e j a$ constante de Planck, e $\gamma$ é o raio giromagnetico o qual é da ordem de (e/mc) para elétrons e (e/Mc) para o núcleo. Quando tal dipolo esta sujeito a campo magnetico $H$, ele sofre uma interaçăo e a equaçăo de movimento e

$$
\mathrm{dG} / \mathrm{dt}=\mu-\mathrm{H}
$$

e portanto

$$
\begin{aligned}
& d G / d t=\gamma G \cdot H \\
& d \mu / d t=\gamma_{\mu} \cdot H
\end{aligned}
$$


cartesiano de coordenadas, como mostra a figura 7 , a soluçăo desta equaçăo pode ser escrita da seguinte forma :

$$
\begin{aligned}
& G_{x}=G \operatorname{sen} \alpha \cos \left(\omega_{-} t+\epsilon\right) \\
& G_{x}=G \operatorname{sen} \alpha \operatorname{sen}\left(\omega_{t} t+\epsilon\right) \\
& G_{x}=G \cos \alpha
\end{aligned}
$$

com equações análogas para $\mu$. O movimento dos vetores $\mu$ e $g$ consiste de uma precessão uniforme em torno de $\mathrm{H}$ com velocidade angular

$$
\omega=-\gamma \mathrm{H} \text {, }
$$

onde o sinal negativo significa que se $\gamma$ e positivo a precessão é no sentido contrario ao definido pelo pseudo-vetor $\mathrm{H}$, e viceversa . A.componente de $G, \mu$ ao longo de $H$ permanece $\dot{r}$ ixa em magnitude, entao a energia do dipolo no campo $H$, que e dada por

$$
W=-\mu \cdot H
$$

é uma constante de movimento.
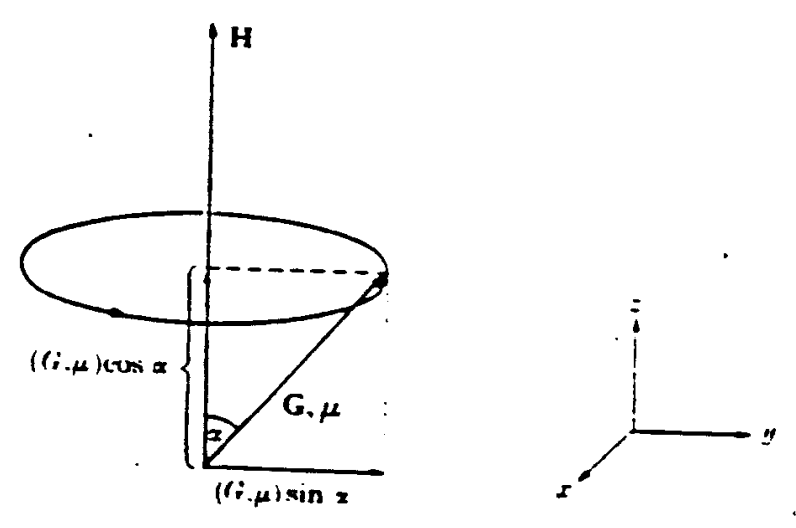

figura 7 - Precessao do dipolo magnetico $\mu$ com momento angular G em torno do campo magnetico aplicado $\mathrm{H}$. A velocidade angular $\omega_{L}=-\gamma H$ é positiva se $\gamma$ é negativo. 
Quando um atomo ou fon tem um momento angular resultante dentro de seu sistema eletrónico, ele possuira um momento de dipolo permanente. O raio giromagnético é então

$$
\gamma=-g(e / 2 m c)
$$

onde o sinal negativo ocorre devido a carga negativa do eletron; e , m são carga e massa do eletron 1 ambos tomados como numeros positivos, . A quantidade g e um numero puro da ordem da unidade, cujo valor depende da contribuiçăo da orbita e do spin do momento agular total. Se apenas o momento orbital está presente $g=g_{L}=1$. Analogamente. se apenas o momento do spin do eletron estiver presente $g=g_{s} \approx 2^{m T}$.

Quando temos um sistema que ambos, momento orbital e momento de spin estão presentes, o valor de $g$ depende da natureza do acoplamento entre eles. No acoplamento spin-orbita. - momento angular resultante está associado com um numero quântico $\mathrm{J}$, onde $\mathrm{J}=\mathrm{L}+\mathrm{S}$, e o valor apropriado de $g$ é

$$
g_{J}=\frac{J(J+1)\left(g_{L}+g_{S}\right)+[L(L+1)-S(S+1)]\left(g_{L}+g_{B S}\right)}{2 J(J+1)}
$$

conhecido como formula de Lande.

0 momento de dipolo magnetico eletronico resultante e

$$
\mu \cdot=-g, B J
$$

onde $B=$ (eh/2mc) é o magneton do Bohr.

\subsubsection{ESTRUTURA FINA}

Quando um atomo ou Ion esta dentro de um campo cristalino seu espaço passa a ter simetria do campo e este fato acarreta mudanças na distribuiçăo de orbitais. Neste caso começa a ser 
valido o conceito de "spin efetivo" $\tilde{\mathrm{S}}$.

No caso de um campo cristalino com simetria cúbica (para a qual ofator $g$ e necessariamente isotropico, $g_{x x}=g_{y x}=g_{z x}$ ) qualquer que seja a direção de $\mathrm{H}$, teremos

$$
W=B(H \cdot g . \tilde{s})
$$

Isto resulta num conjunto de niveis igualmente espaçados, e transições permitidas entre niveis sucessivos $\left(\hat{A} \mathbb{M}_{m}= \pm 1\right)$, os quais diferem em energia de $\mathrm{gBH}$. Desta forma, as $2 \tilde{\mathrm{S}}$ transiçoes devem obedecer a seguinte equação :

$$
h \gamma=g B H
$$

Na figura 8 temos o efieito Zeeman para o caso de $\tilde{\mathrm{S}}=1$. e o caso do $\mathrm{Ni}^{+=}$, $3 d^{\ominus}$, em um campo ligante de simetria exatamente octaedrica.

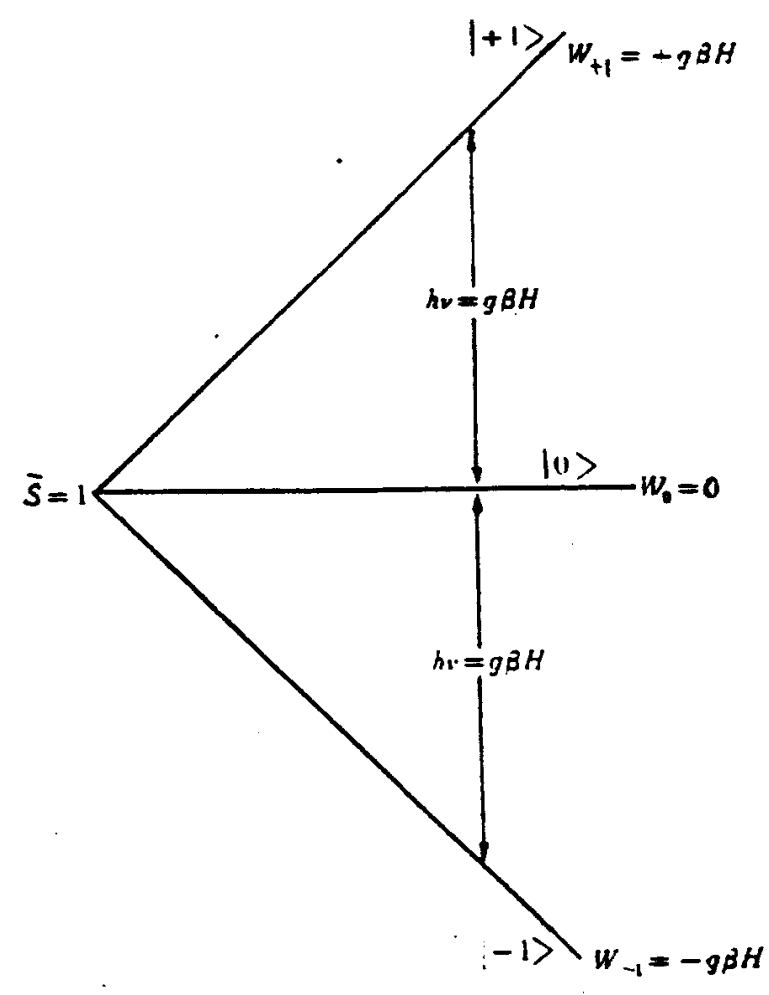

figura 8 - Efeito Zeeman para o estado $\tilde{S}=1$, na ausencia de qualquer desdobramento inicial dos niveis. As duas transiçסes via ressonancia magnética permitidas coincidem com $h \nu=g B H$. 
Se a simetria na fosse cubica, os tres niveis poderiam nao estar degenerados num campo magnetico zero.

No caso de simetrias menores que cubica a Hamiltoniana de spin efetivo ganha mais termos e o efeito apresenta resultados diferentes, mas a discussão destes têrmos e dos eíeitos que eles acarretam năo sera feita neste trabalho. Na referencia pode-se encontrar o assunto em detalhe.

\subsubsection{ESPECTRO DE RPE}

O espectro de ressonancia paramagnetica eletronica e obtido atraves da aplicaçăo de um campo magnetico $\mathrm{H}$ sobre a amostra em estudo para que os elétrons possam interagir com este campo e se - estado em que ele se encontra apresentar degenerescencia de spin o sistema sofrera o efeito Zeeman. Com os niveis de energia já abertos começa-se a jogar um espectro de frequèncias de baixa energia ate observar-se a ressonancia com uma transição do sistema e procedendo-se desta forma continuamente obtem-se 0 espectro de RPE. 


\section{CAPITULO III - TECNICAS EXPERIMENTAIS}

\subsection{PREPARACAO DAS AMOSTRAS}

Os cristais, em geral, foram cortados e polidos na oricina de otica do DFCM-IFQSC. Apenas quando a utilização da oficina fosse acarretar algum atraso em nossos trabalhos é que iazlamos o corte e polimento em nosso laboratorio, pois tambem possulamos uma serra apropriada para corte de cristais pequenos e material para o polimento.

A maior dificuldade no preparo das amostras é o polimento pois o LiNbos é um cristal duro ( seu grau de dureza e 4.5 Mohs) e seu polimento exige tecnica e paciência.

\subsubsection{CORTE}

Para nossos estudos e necessario possuirmos cristais com eixo c ( ou z) paralelo e perpendicular a face. A direção de crescimento do niobato de litio é a do eixo $z$, portanto para obtermos os cristais com sistema de eixos desejados cortamos o tarugo do cristal em rodelas de aproximadamente $0.5 \mathrm{~cm}$ de espessura e $3 \mathrm{~cm}$ de diametro, que ificavam contidas no plano [001] . Esta configuraçăo de corte tambem nos assegurava uma concentração bem homogenea para todas as amostras . Estas amostras eram divididas em quatro pedaços a fim de se obter paralelepipedos com o eixo c perpendicular a face maior. 
Finalmente, estes paralelepipedos eram cortados em fatias de aproximadamente $0.8 \mathrm{~mm}$ de espessura, na direção [001] e [010].

\section{1 .2 POLIMENTO}

No inicio do processo de polimento é feito a lapidaça com Iixas 180 e 600 . Depois usa-se po carburundum 800 e 2000 Terminada esta fase, passa-se a usar po de alumina e etileno glicol para lubrificar. A granulaçao inicial do po de alumina e 5 um e vai decrescendo até terminar o polimento com 0.05 um.

\subsection{DEWAR UTILIZADO PARA MEDIDAS A BAIXA TEMPERATURA}

O dewar utilizado nas medidas a temperatura de nitrogênio liquido foi desenvolvido em noso departamento. Na figura 9 temos uma foto do dewar e na figura 10 temos um esquema da sua parte interna.

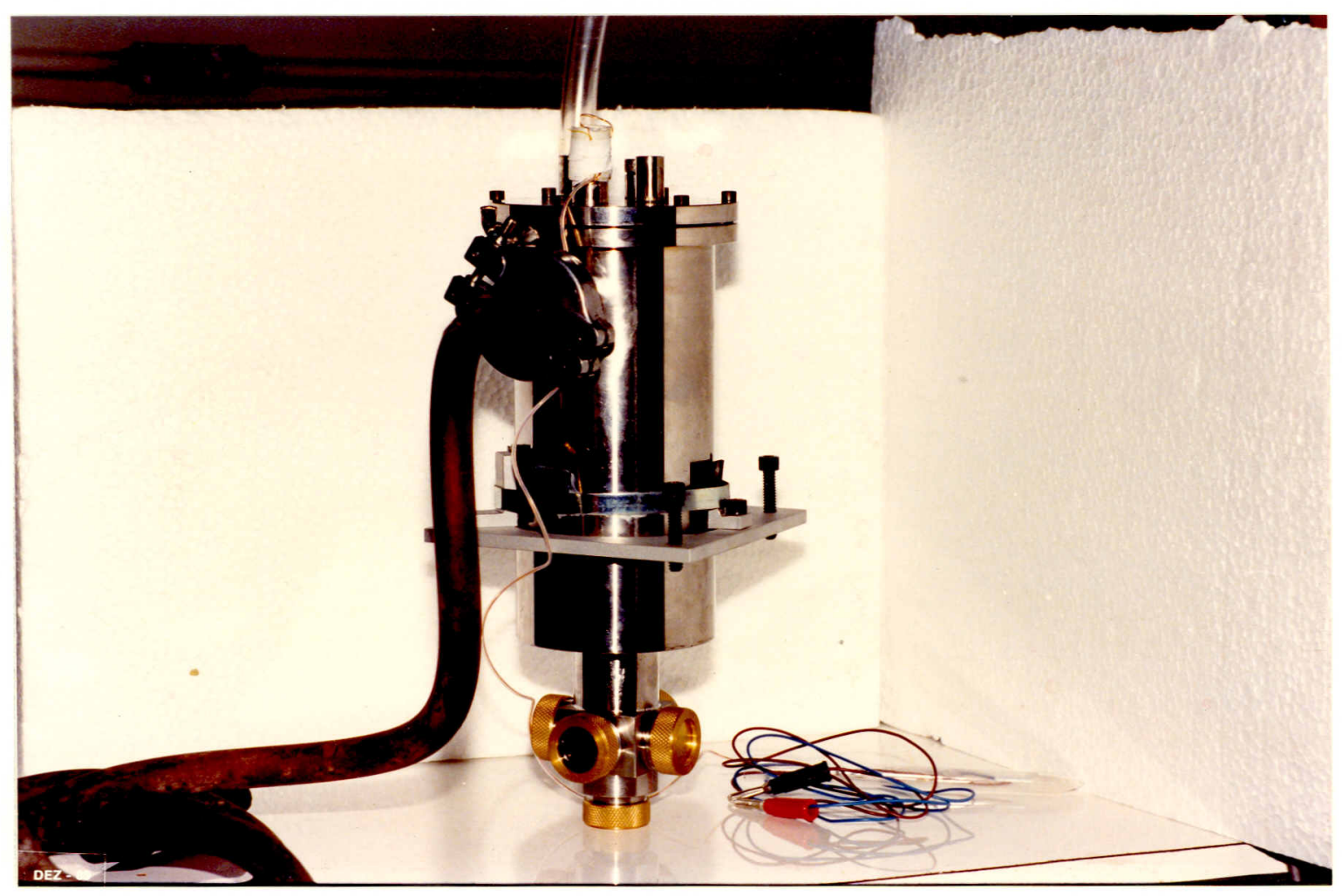

figura 9 - Dewar utilizado nas medidas à baixa temperatura 


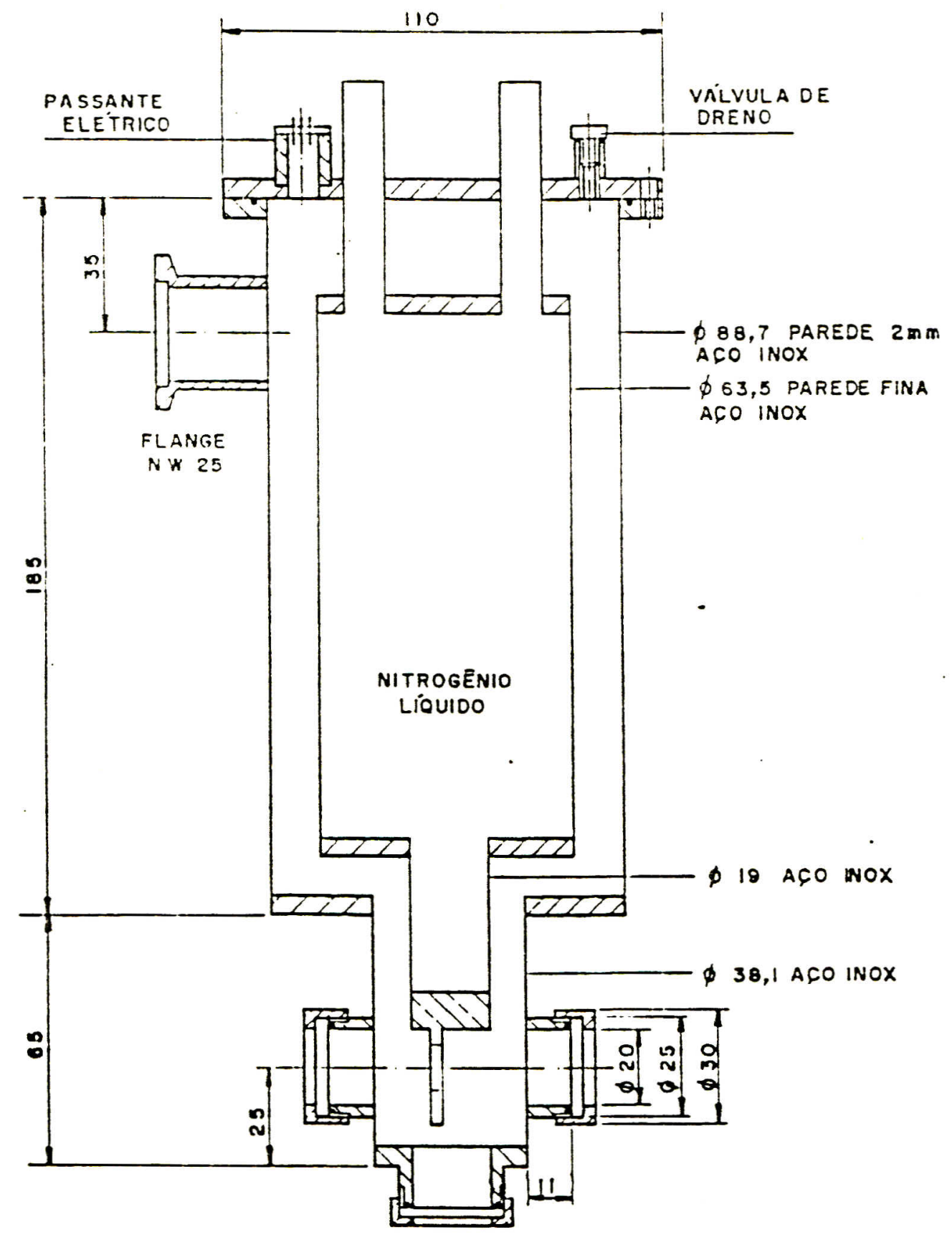

figura 10 - Corte transversal do dewar utilizado 


\section{3 ABSORÇAO ÓTICA}

As medidas de absorçăo otica foram feitas a temperatura de nitrogênio liquido utilizando o dewar descrito na seçăo 3.2 .

Os espectros de absorçăo polarizada entre 0.3 a 2.0 um (33333 a $5000 \mathrm{~cm}^{-1}$ ) foram medidos usando um espectrofotometro Cary-17 com polarizador tipo Glan-Thompson de calcita. A resolução espectral limite foi $0.03 \mathrm{~nm}$ no visivel e $1.0 \mathrm{~nm}$ na regiåo do infravermelho proximo. Na regiao do infravermelho medio, ou seja, de 2.5 a 50 um ( 4000 $200 \mathrm{~cm}^{-1}$ ), utilizamos um espectrofotômetro Perkin-Elmer 180, e a resoluçå espectral limite foi $1 \mathrm{~cm}^{-1}$.

\subsection{RESSONÀNCIA PARAMAGNETICA ELETRÔNICA}

Os espectros de RPE foram obtidos utilizando-se o espectrômetro Varian Banda-X ( $9 \mathrm{GHz})$, do grupo de biofisica do DFCM-IFOSC . A modulaçăo do sinal foi feita em $100 \mathrm{KHz}$ e a potência de microondas $10 \mathrm{~mW}$.

\subsection{TRATAMENTO TERMICO}

\subsubsection{INTRODUCAO}

o procedimento utilizado para acompanhar o comportamento das concentraçðes de $\mathrm{Fe}^{+2}, \mathrm{Fe}^{+x}$ e $\mathrm{OH}^{-}$foi o seguinte : para uma dada temperatura do forno deixava-se o cristal durante um determinado periodo de tempo ( dependendo da amostra ) , resfriava-se o cristal rapidamente, faziam-se as medidas de absorçăo otica e RPE, depois colocava-se o cristal novamente no forno e repetia-se o procedimento. 


\subsubsection{TRATAMENTO TERMICO NO AR}

Os tratamentos termicos no ar podem ser divididos em duas partes :
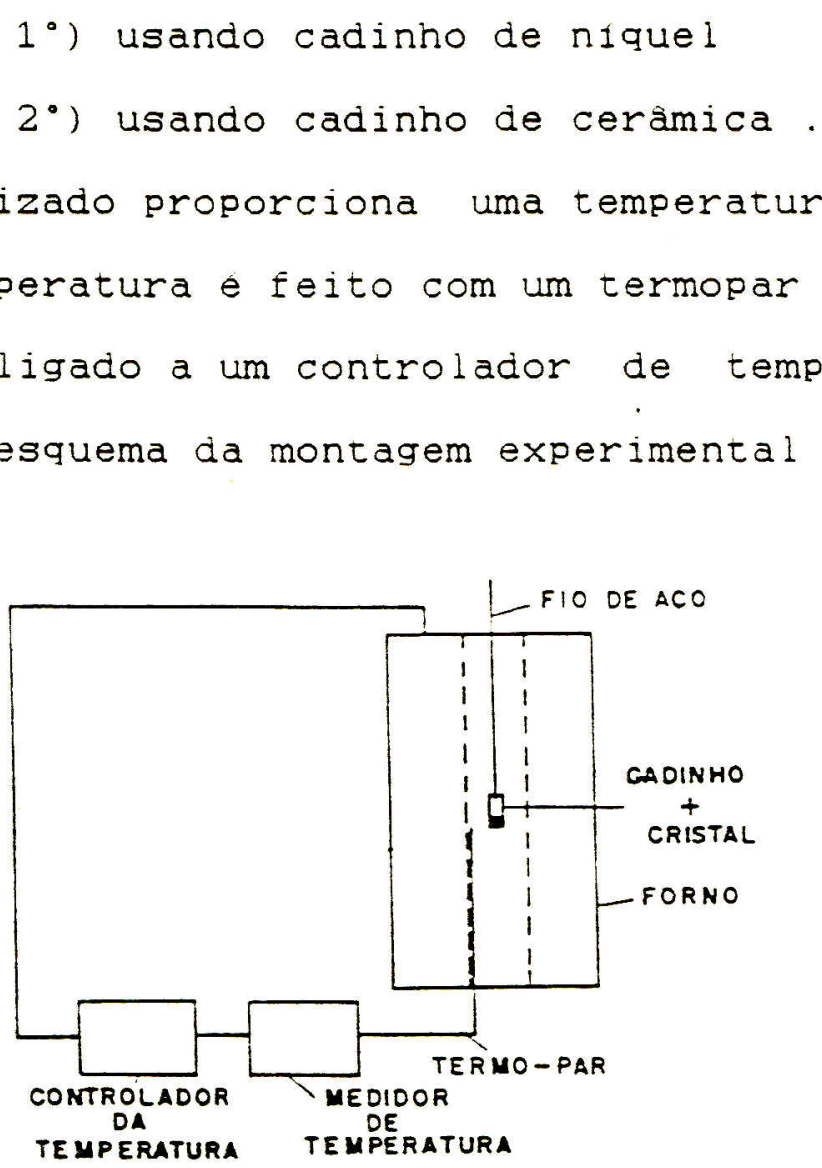

figura 11 - Montagem experimental para o tratamento termico no ar

\subsubsection{TRATAMENTO TERMICO EM ATMOSFERA DE OXIGÊNIO}

No tratamento termico em atmosfera de oxigènio usamos um cadinho de ceramica dentro de um tubo horizontal por onde passa o fluxo de oxigenio. Este tubo e colocado dentro do forno como mostra a figura 12 . O fluxo de oxigenio e controlado na salda do tubo pela irequencia das bolhas no recipiente com oleo colocado ao lado da montagem. O controle da temperatura foi feito da mesma maneira que na montagem anterior. 


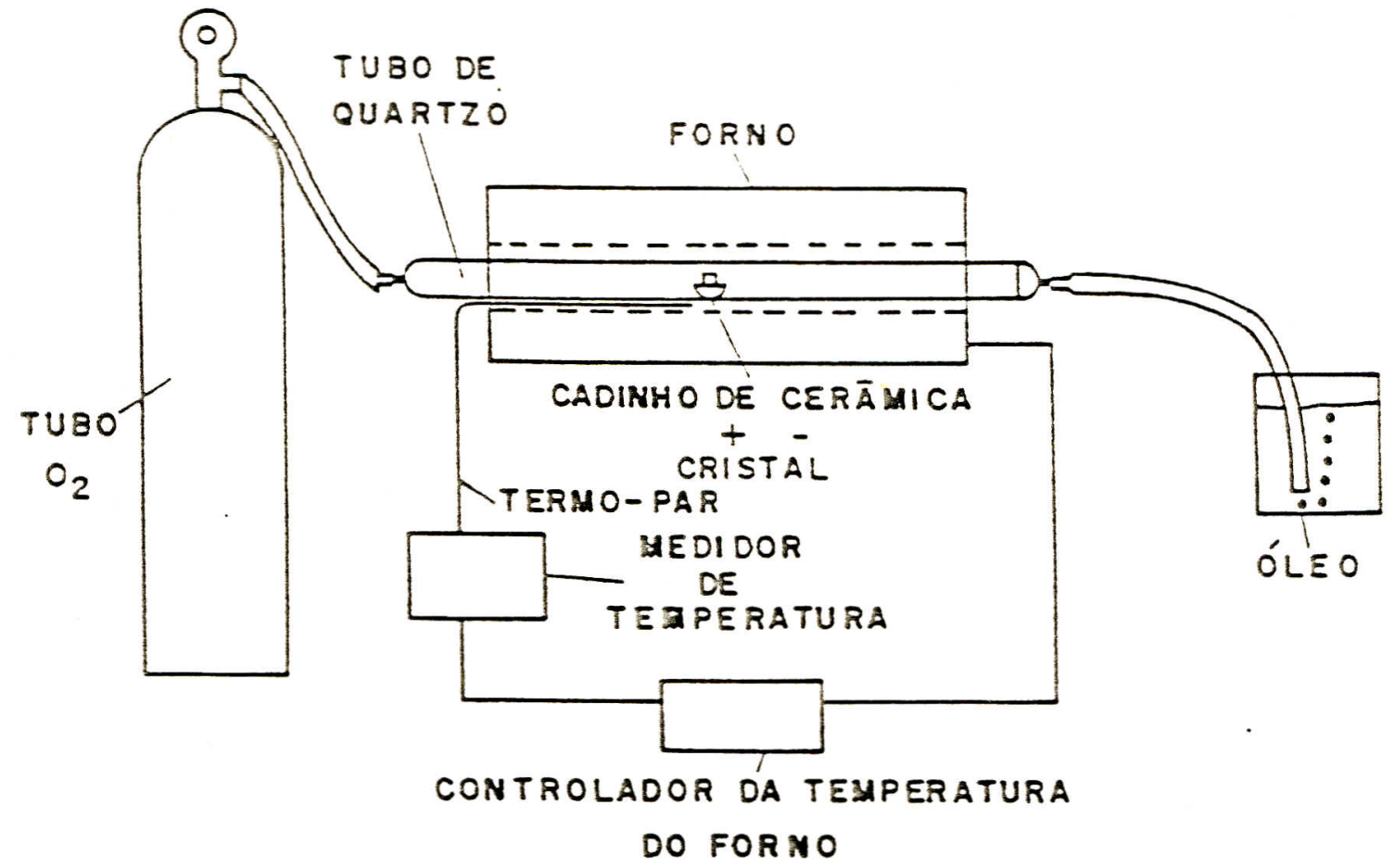

figura 12 - Montagem experimental para tratamento termico em atmosfera de oxigenio.

\subsection{LUMINESCÊNCIA}

As medidas de luminescencia polarizada foram feitas a tempertura ambiente e de nitrogênio liquido utilizando a montagem experimental mostrada nas figuras 13,14 e 15 .

A resoluçăo maxima oferecida pela montagem e $0.5 \mathrm{~nm}$ na regiă do visivel. 


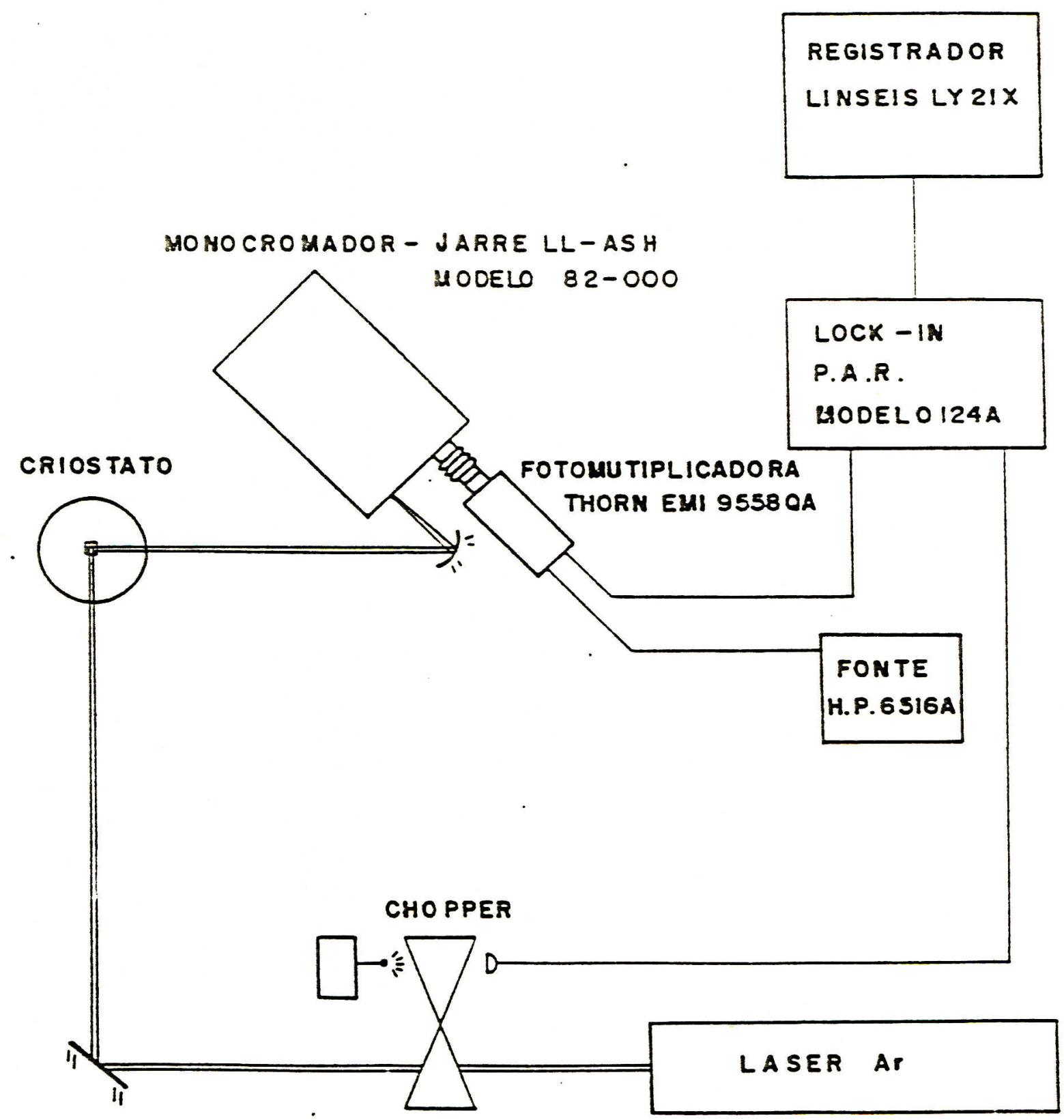

figura 13 - Montagem experimental para medida do espectro de luminescência . 


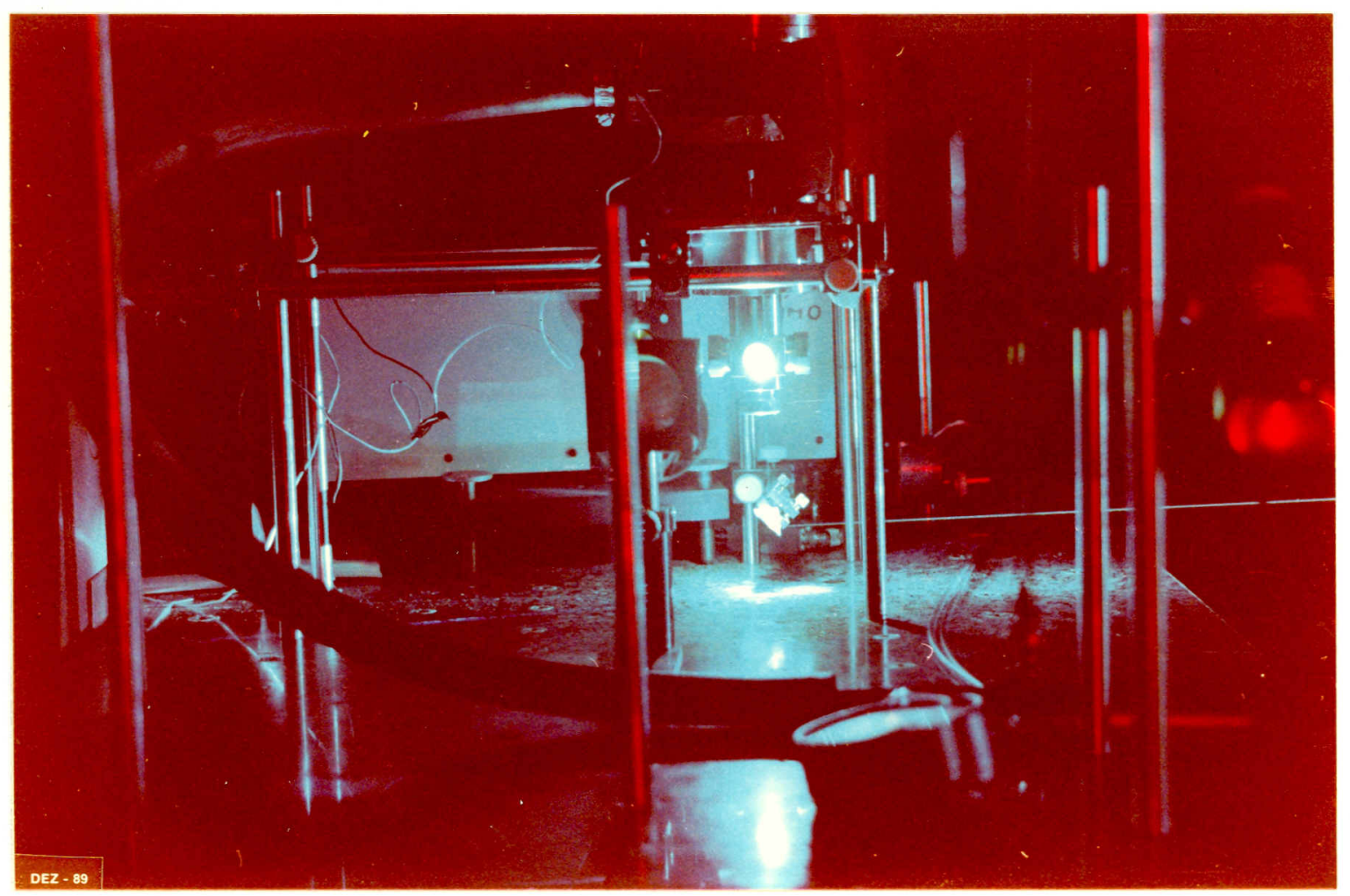

(d)

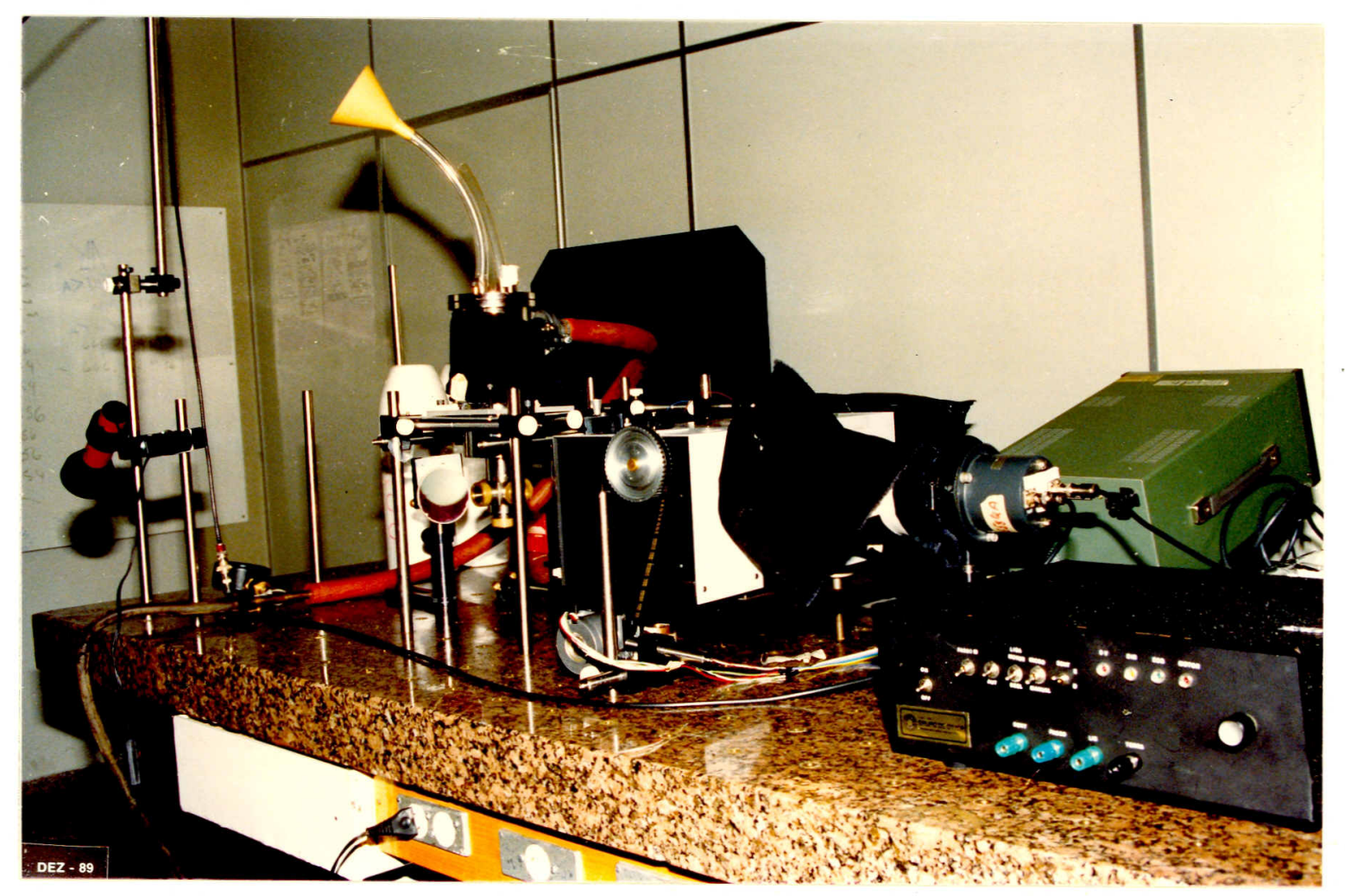

(b)

figura 14 - Fotos da montagem experimental para medir luminescência : (a) bombeamento com laser de argónio

(b) deteçåo do sinal. 

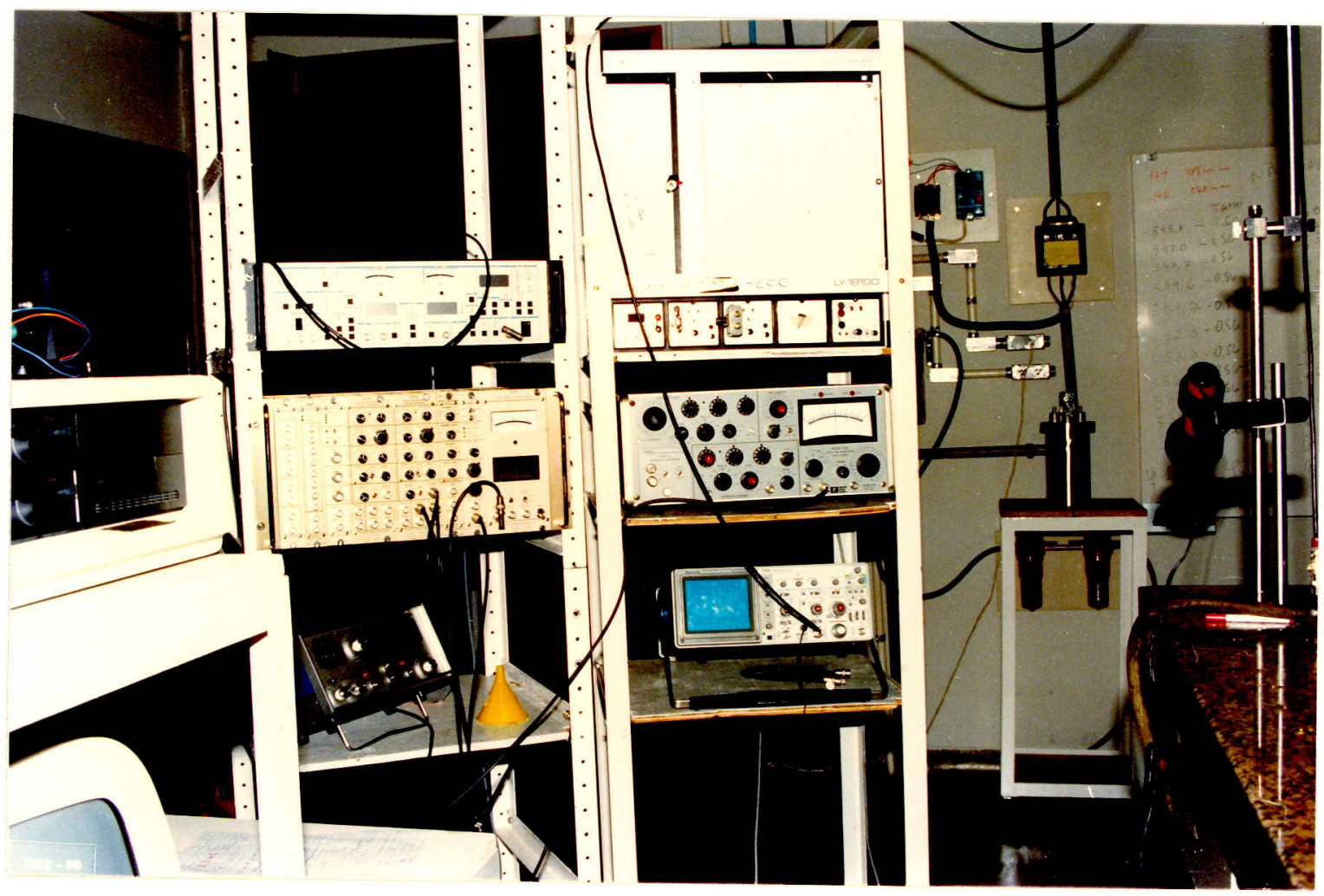

(a)

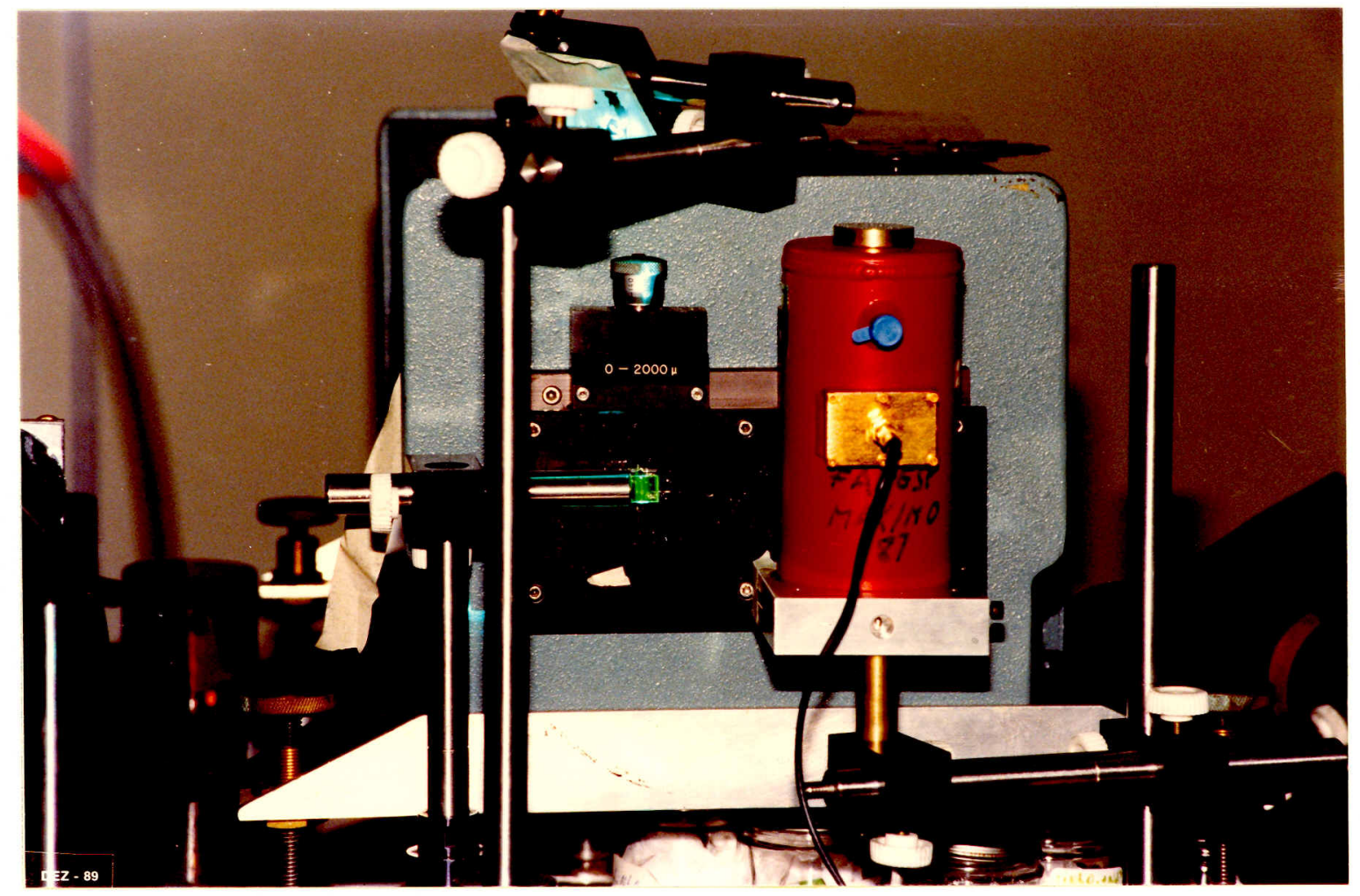

(b)

figura 15 - Fotos da montagem experimental para medir luminescência : (a) sistema de aquisiça de dados

(b) luminescencia à temperatura ambiente. 


\subsection{MEDIDA DO TEMPO DE VIDA DOS ESTADOS LUMINESCENTES}

As medidas do tempo de vida de luminescència foram feitas a temperatura ambiente e 77K utilizando-se a montagem experimental mostrada na figura 16.

A polarização usada na excitaça foi $\propto$.

o sistema de bloqueio da luz foi feito atraves do chopper. Para minimizar o tempo de decaimento da propria montagem focalizamos a luz do laser nas extremidades da pa do chopper e colocamos o chopper em rotaça maxima $(\sim 150 \mathrm{~Hz})$. Desta forma 0 feixe, agora com diametro da ordem de décimos de milimetros. passou a ser cortado com uma alta velocidade tangencial. Podemos fazer uma avaliaçăo rapida da ordem de grandeza deste tempo de decaimento. O raio do chopper é aproximadamente $7 \mathrm{~cm}$ e 0 feixe do laser aproximadamente $0.1 \mathrm{~mm}$. Isto significa que o tempo de corte do sinal luminoso é em torno de $10 \mathrm{us}$. Alem deste tempo temos que levar em conta tambem o tempo de resposta eletronica do sistema que pode interierir neste resultado.

o resto da montagem năo tem muita coisa nova em relaçao au da medida de luminescencia a unica coisa que mudou é na salda do sinal da foto que agora esta ligada a um osciloscopio. 


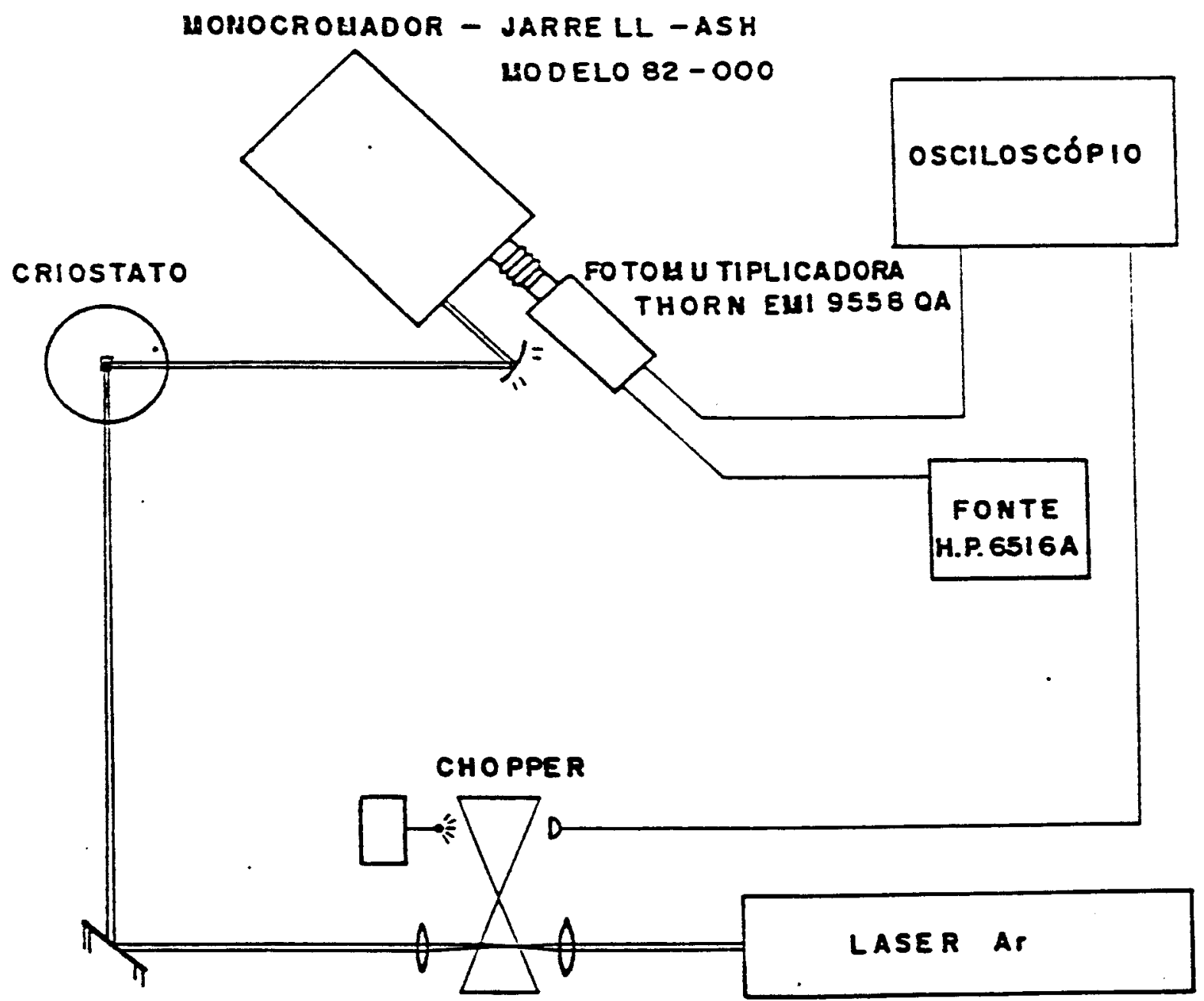

figura 16 - Montagem experimental para medida do tempo de vida de luminescência. 


\section{CAPITULO IV - RESULTADOS EXPERIMENTAIS}

\subsection{INTRODUSAO}

Este capitulo esta dividido em quatro partes : 4.2 Tratamentos Térmicos, 4.3 Absorçă Polarizada, 4.4 Emisså Polarizada e 4.5 Tempo de Vida. Na seção 4.2 , que está destinada aos tratamentos térmicos, mostramos o efeito da temperatura nas concentraçסes de $\mathrm{Fe}^{+2}$, $\mathrm{Fe}^{+*}$ e $\mathrm{OH}^{-}$através de medidas de absorção otica e RPE. Na seção 4.3 temos os resultados obtidos para a absorção polarizada medidos a temperatura de nitrogènio liquido e na seção 4.4 temos os resultados emissão polarizada medidos a temperatura ambiente e $77 K$. Finalmente na seção 4.5 temos as medidas de tempo de vida feitas com polarização $\propto$ a temperatura ambiente e 77K.

\subsection{TRATAMENTOS TERMICOS}

\subsubsection{TRATAMENTO TERMICO NO AR}

- tratamento termico no ar foi feito em amostras puras e dopadas com dois cadinhos diferentes cadinhos : cadinho de niquel e cadinho de ceramica. Com o cadinho de ceramica ná obtivemos bons resultados: tanto para uma amostra de niobato de l1tio puro quanto para una dopada com erbio era necessario muitas horas para uma mudança pouco relevante das concentraçőes 
de $\mathrm{Fe}^{+2}$ e $\mathrm{OH}^{-}$.

Usando o cadinho de niquel fizemos tratamentos fixando 0 tempo e variando a temperatura de 500 a $900^{\circ} \mathrm{C}$, e a maior eficiencia do tratamento foi obtida para a temperatura de $700^{\circ} \mathrm{C}$. Este resultado tambem foi obtido por Mastelaroin no seu estudo sobre a caracterização do LiNbO= puro; na figura 17 temos o resultado obtido por ele. Desta forma, para caracterizar a dependência da concetração com o tempo de tratamento utilizamos a temperatura de $700^{\circ} \mathrm{C}$.

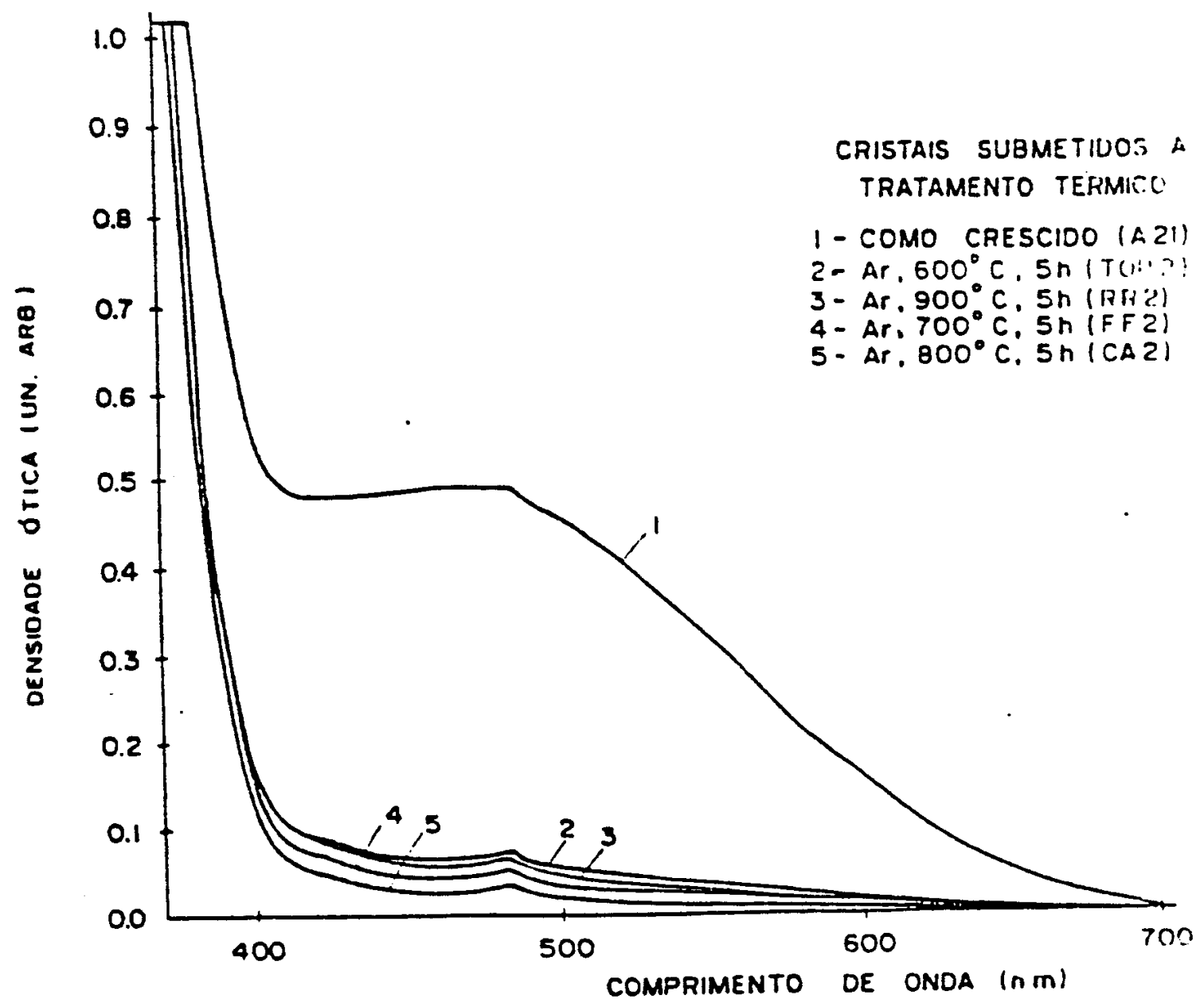

figura 17 - Espectro no visivel mostrando a variaçăo da banda de absorça do $\mathrm{Fe}^{+z}$ no LiNbo-s:Fe após tratamento térmico em atmosfera ambiente.

Com o cadinho de niquel notamos que os processos de reduça das concentraçres de $\mathrm{OH}^{-}$e $\mathrm{Fe}^{+2}$ foram muito mais rapidos $e$ 
eficientes do que no cadinho de ceramica. Na figura 18 temos o comportamento da absorção do $\mathrm{OH}^{-}$em $2.7 \mu \mathrm{m}$ com os consecutivos tratamentos térmicos feitos a $700^{\circ} \mathrm{C}$ com o cadinho de niquel para uma amostra pura e outra com erbio.

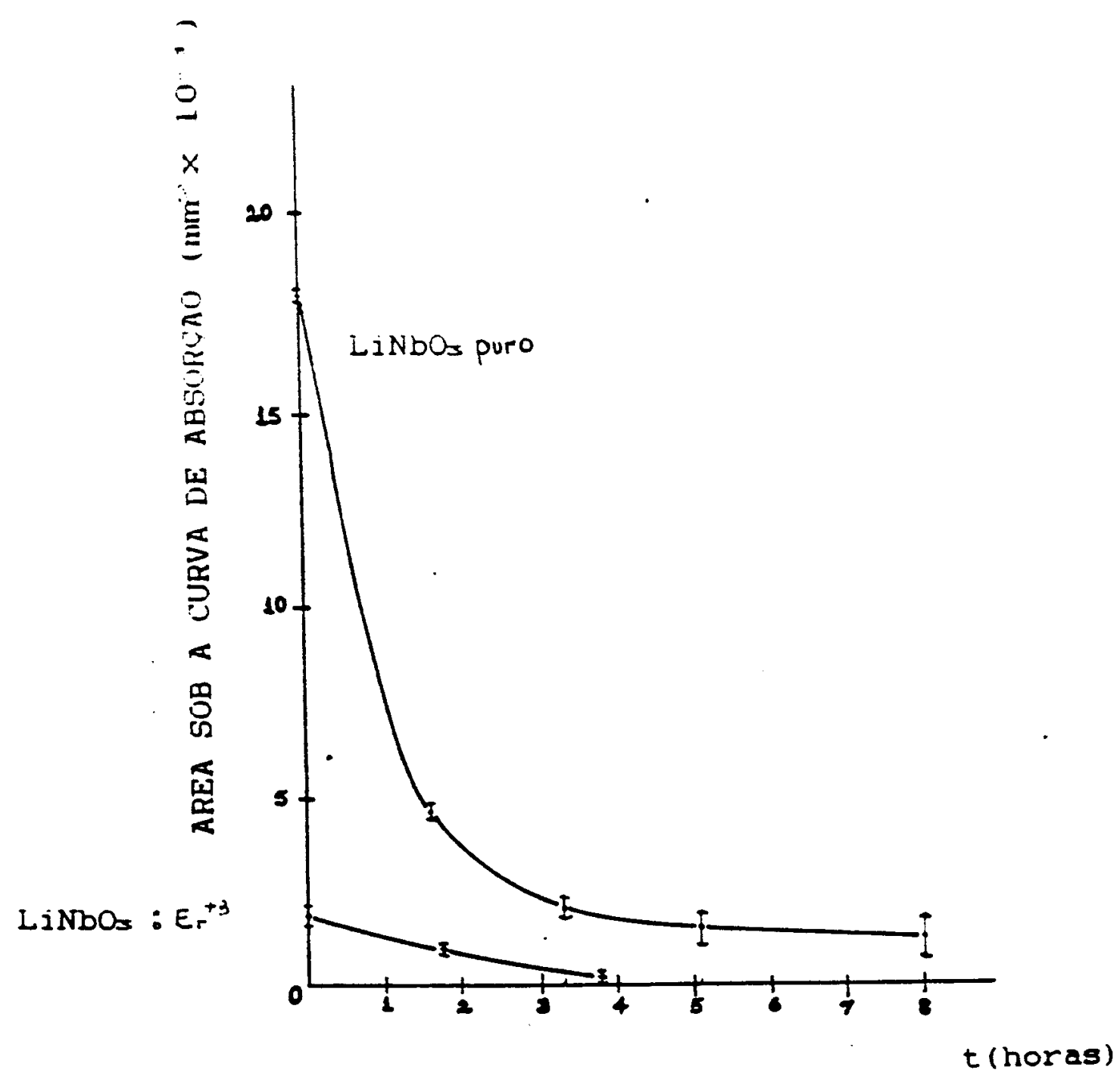

figura 18 - Grafico do comportamento da concentraçăo de $\mathrm{OH}^{-}$em funçå do tempo durante os tratamentos térmicos.

Paralelamente foram feitas medidas do comportamento da concentraçăo de $\mathrm{Fe}^{+z}$, atraves da banda em $485 \mathrm{~nm}$, com os consecutivos tratamentos térmicos. Os resultados relativos ao cadinho de niquel estao na figura 19 . Nå foram detectadas alterações na concentraçăo de $\mathrm{Fe}^{+\infty}$ medida através do espectro de RPE

Apesar do tratamento térmico no cadinho de niquel ser mais 
rapido e mais eficiente, ele apresenta um, incoveniente; apos o término do tratamento. os cristais adquirem uma coloraçåo amarela.

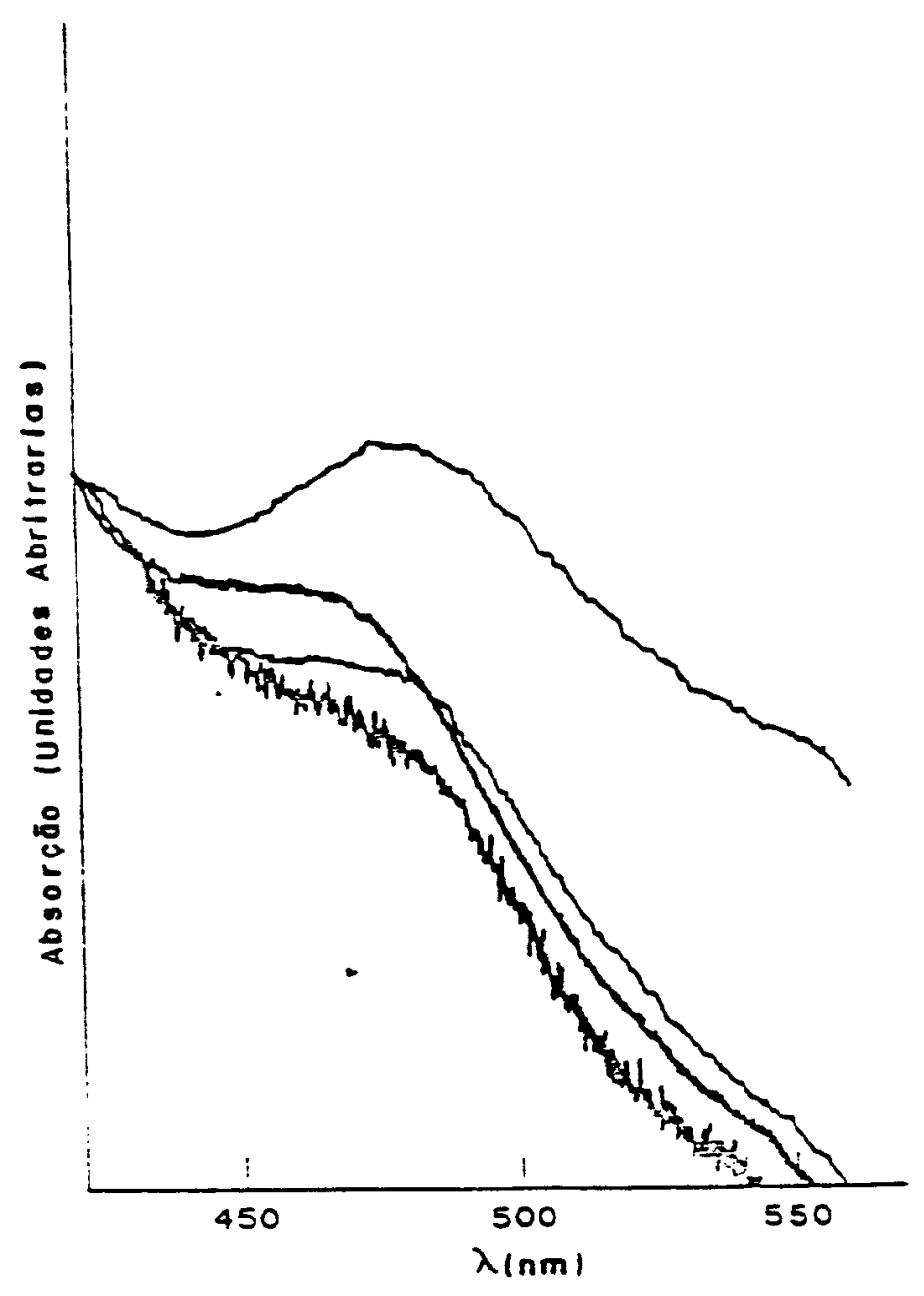

figura 19 - Comportamento da absorção otica relativa à concentraçao de $\mathrm{Fe}^{+2}$ durante os tratamentos térmicos realizados a $700^{\circ} \mathrm{C}$ em atmosfera ambiente com cadinho de niquel.

\subsubsection{TRATAMENTO TERMICO EM ATMOSFERA DE OXIGÊNIO}

Para o tratamento térmico em atmosfera de oxigènio procedemos da mesma forma que no tratamento térmico no ar.

O resultado foi bastante satisfatorio pois consegrimos uma eficiencia proxima à do tratamento feito com o cadinho de niquel no ar, mas sem alterar a coloraça do cristal. Os resultados eståo nas figuras 20 e 21 . 


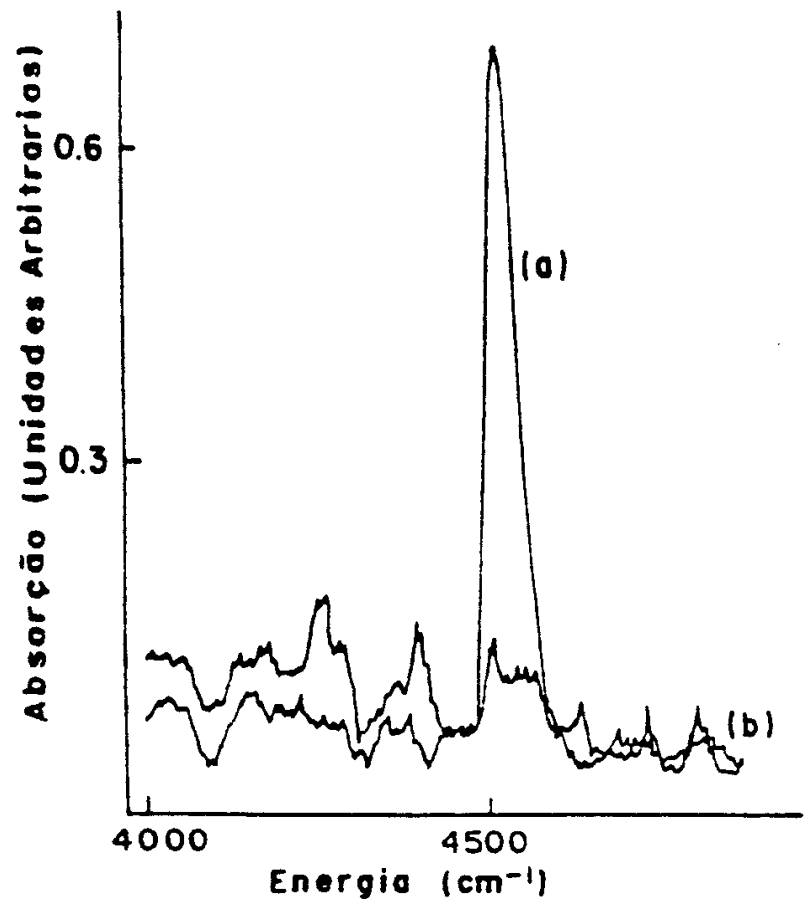

figura 20 - Comportamento da absorção relativa a molécula de $\mathrm{OH}^{-}$ com o tratamento térmico realizado a $700^{\circ} \mathrm{C}$ numa atmosfera de oxigenio durante um periodo de 8 horas.

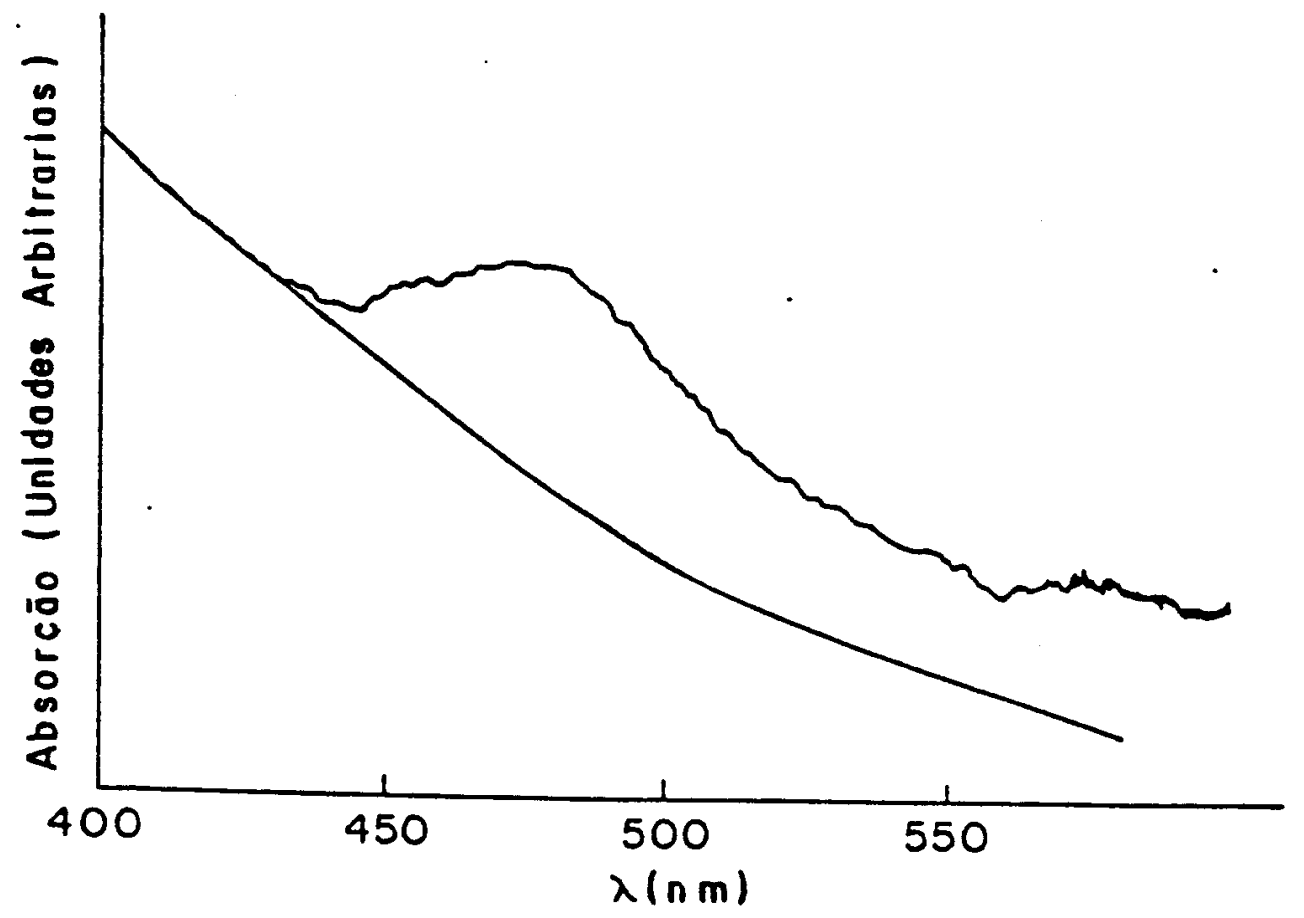

figura 21 - Comportamento da absorção relativa ao ion $\mathrm{Fe}^{+z}$ com o tratamento térmico realizado a $700^{\circ} \mathrm{C}$ numa atmosfera de oxigenio durante um periodo de 8 horas. 


\subsection{ABSORÇAO POLARIZADA}

Nas figuras 22 a 37 temos os espectros de absorçå polarizada na regiå de 350 a 1600 nm à temperatura de nitrogenio liquido . Devido a fraca intensidade das linhas quando o polarizador era colocado, as transiçôs na regiao de 1,0 e 1,5 um so foram medidas com polarização $\propto$.

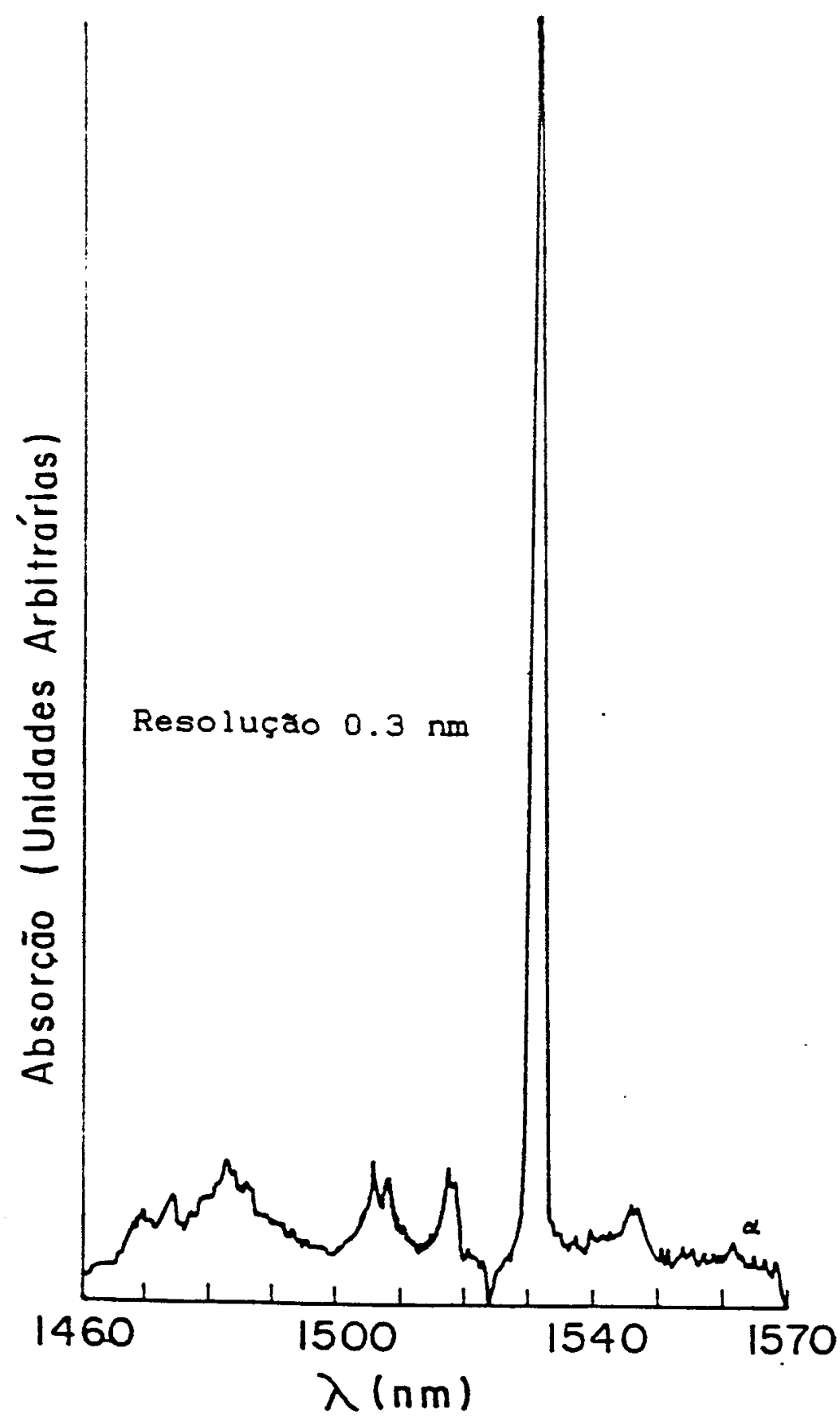

figura 22 - Transiçåo $4 I_{1=12 \rightarrow} \rightarrow I_{13 / 2}$ com polarizaçåo $\alpha$ a $77 \mathrm{~K}$. 


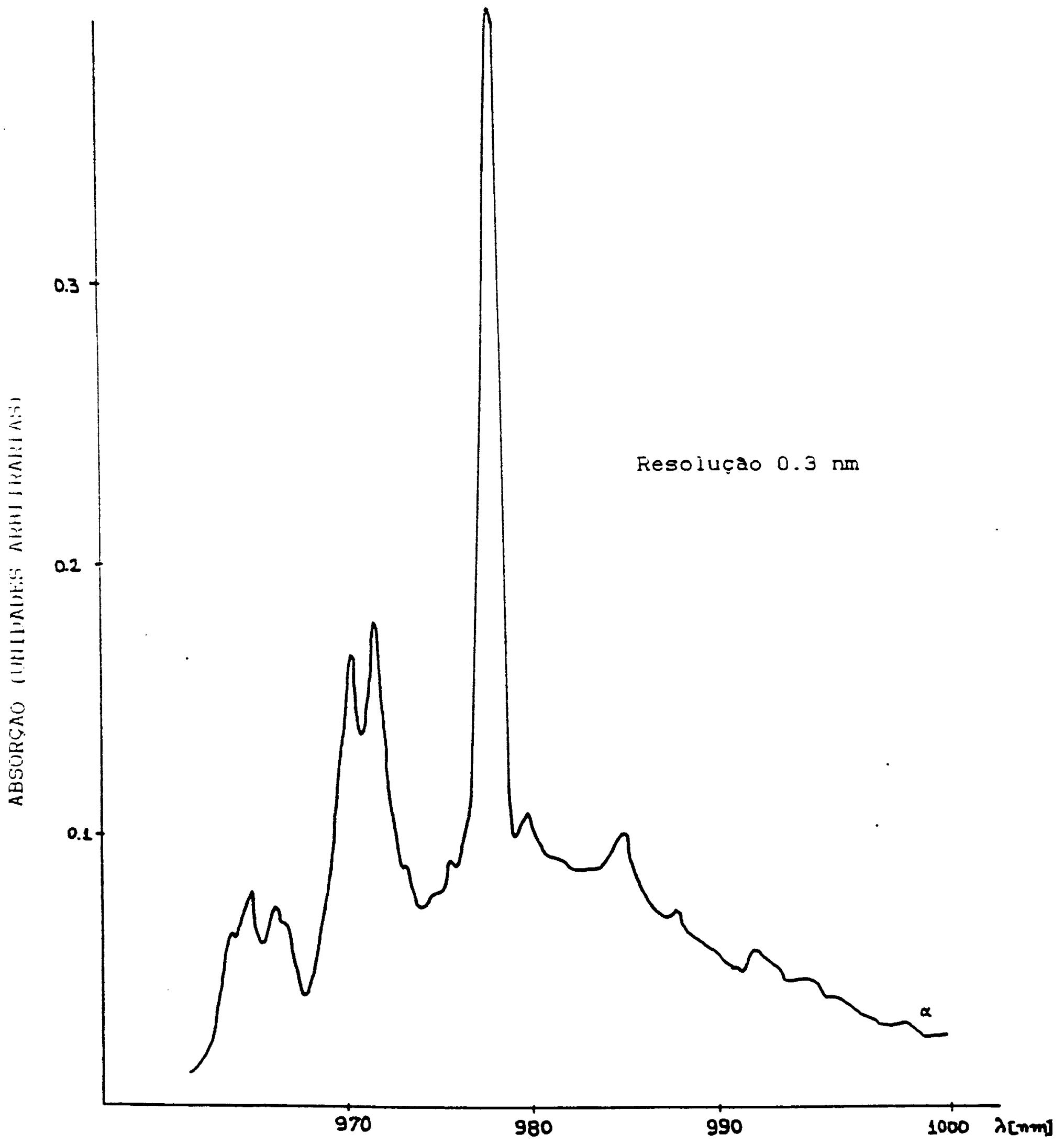

figura 23 - Transiçåo $4 I_{1=/ z} \rightarrow{ }^{4} I_{11 / 2}$ com polarizaçăo $\alpha$ a $77 \mathrm{~K}$. 


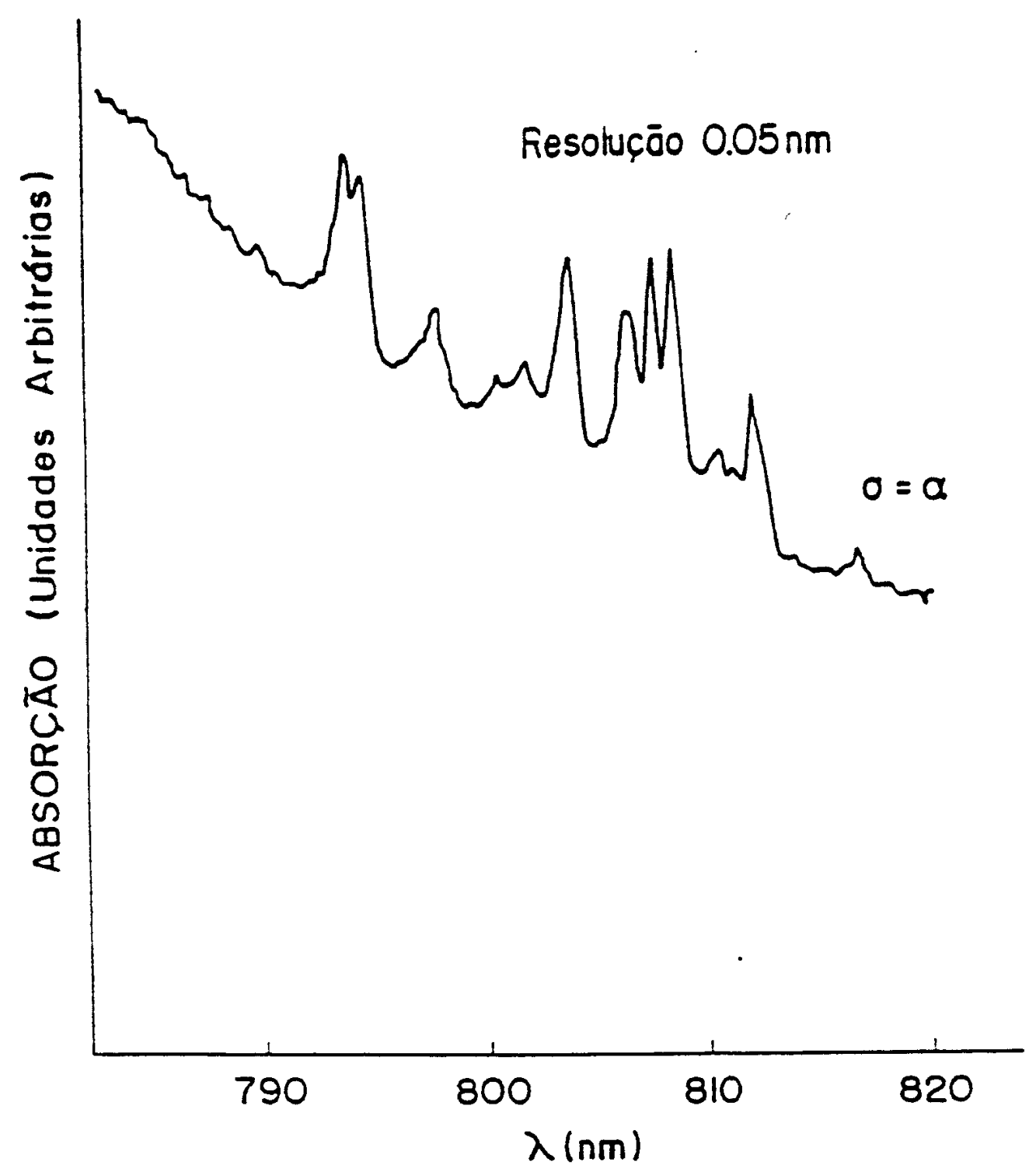

figura 24 - Transição ${ }^{4} \mathrm{I}_{13 / 2} \rightarrow 4 \mathrm{I}_{\infty / 2}$ com polarização $\sigma \alpha$ a $77 \mathrm{~K}$.

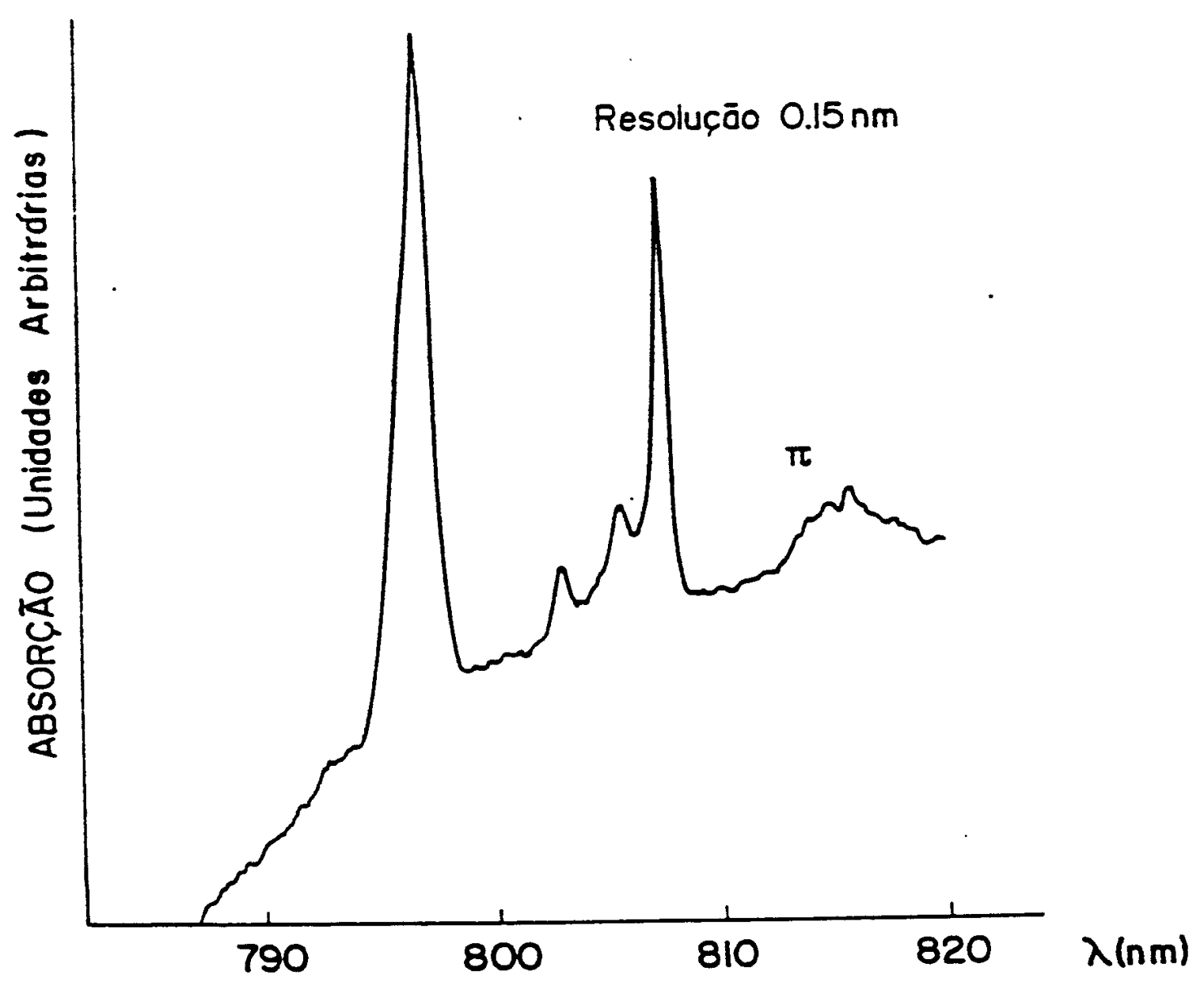

figura 25 - Transiçăo $4 I_{1=12} \rightarrow I_{41}=$ com polarizaçăo $\pi$ a $77 \mathrm{~K}$. 

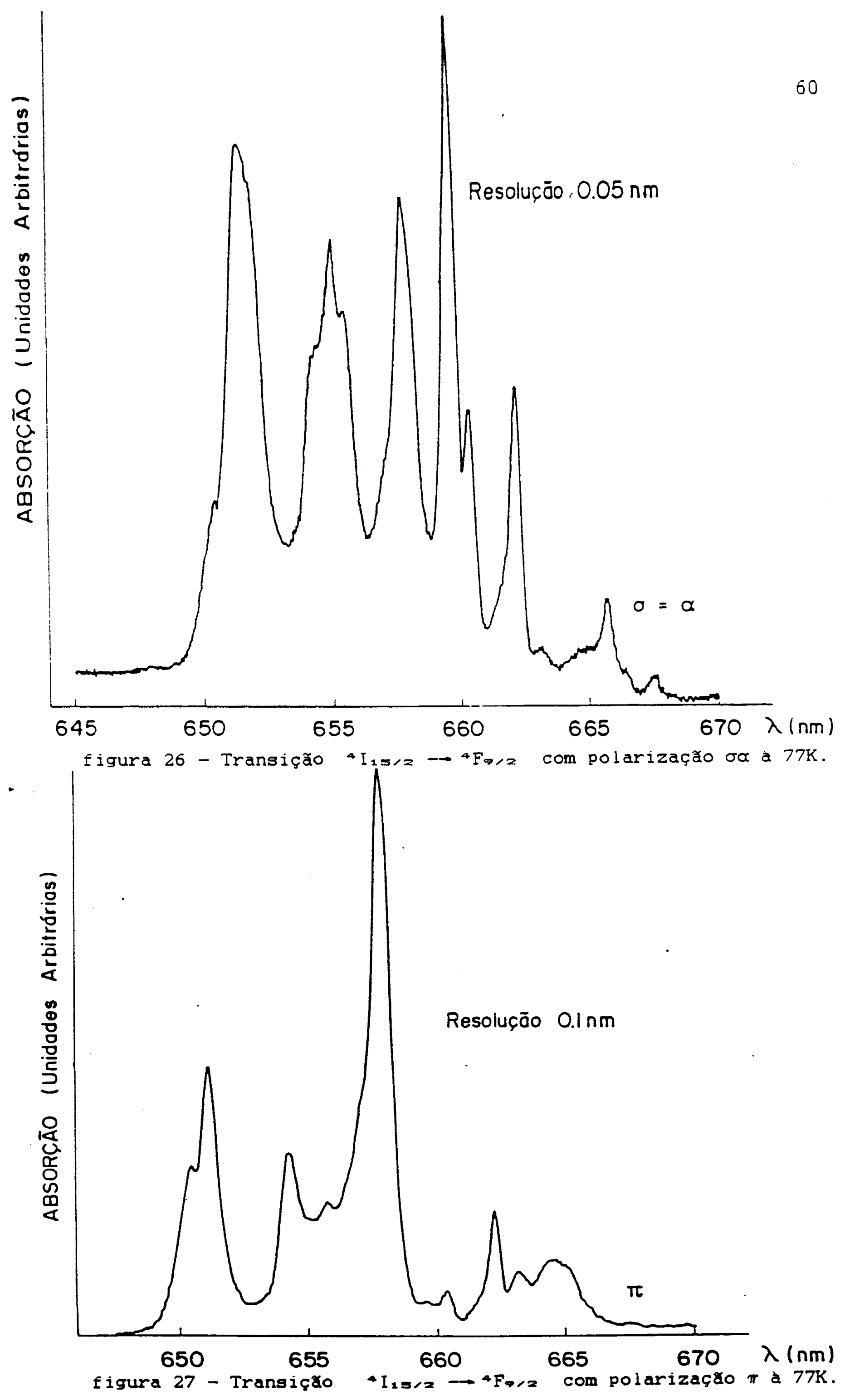


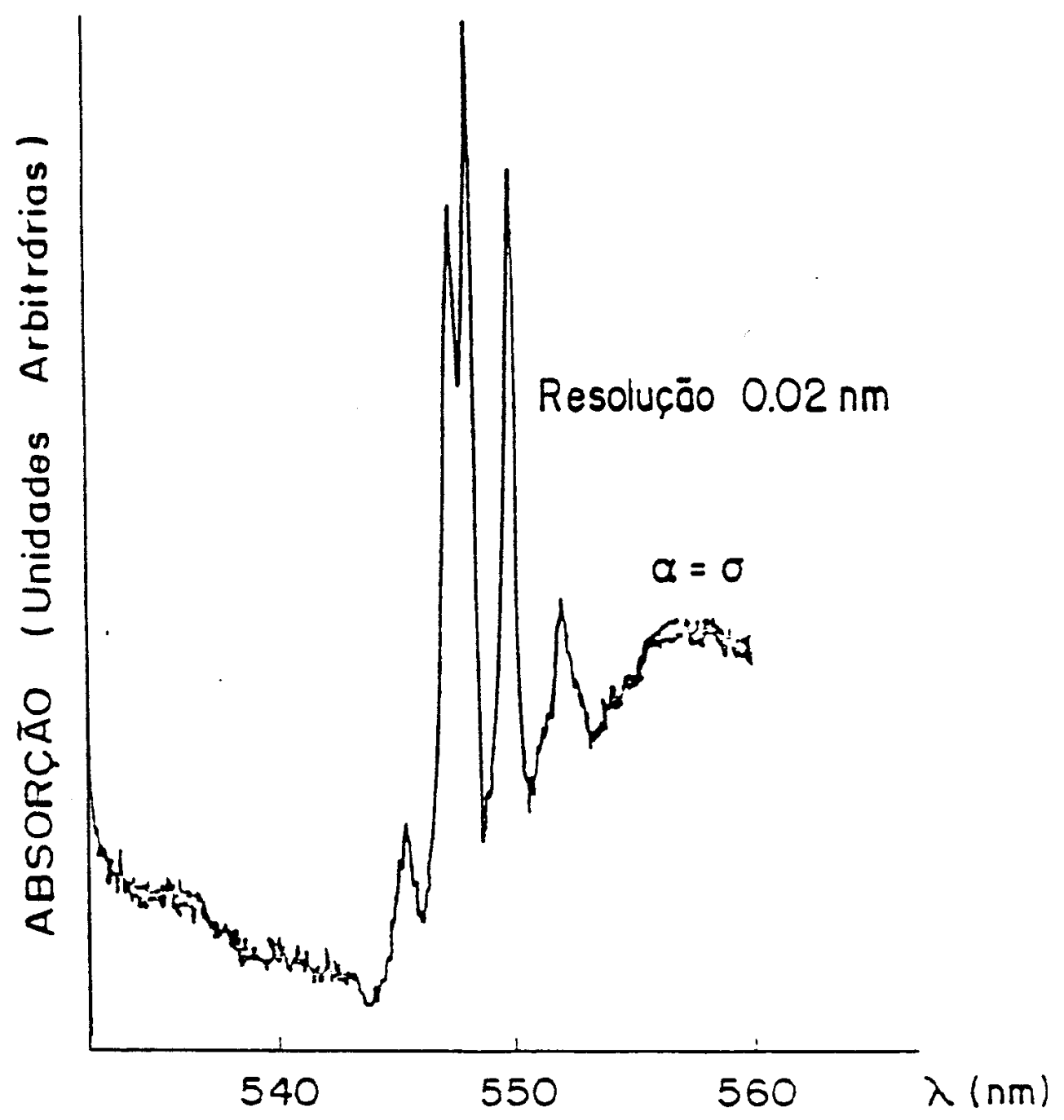

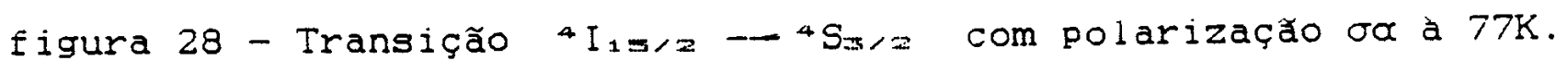

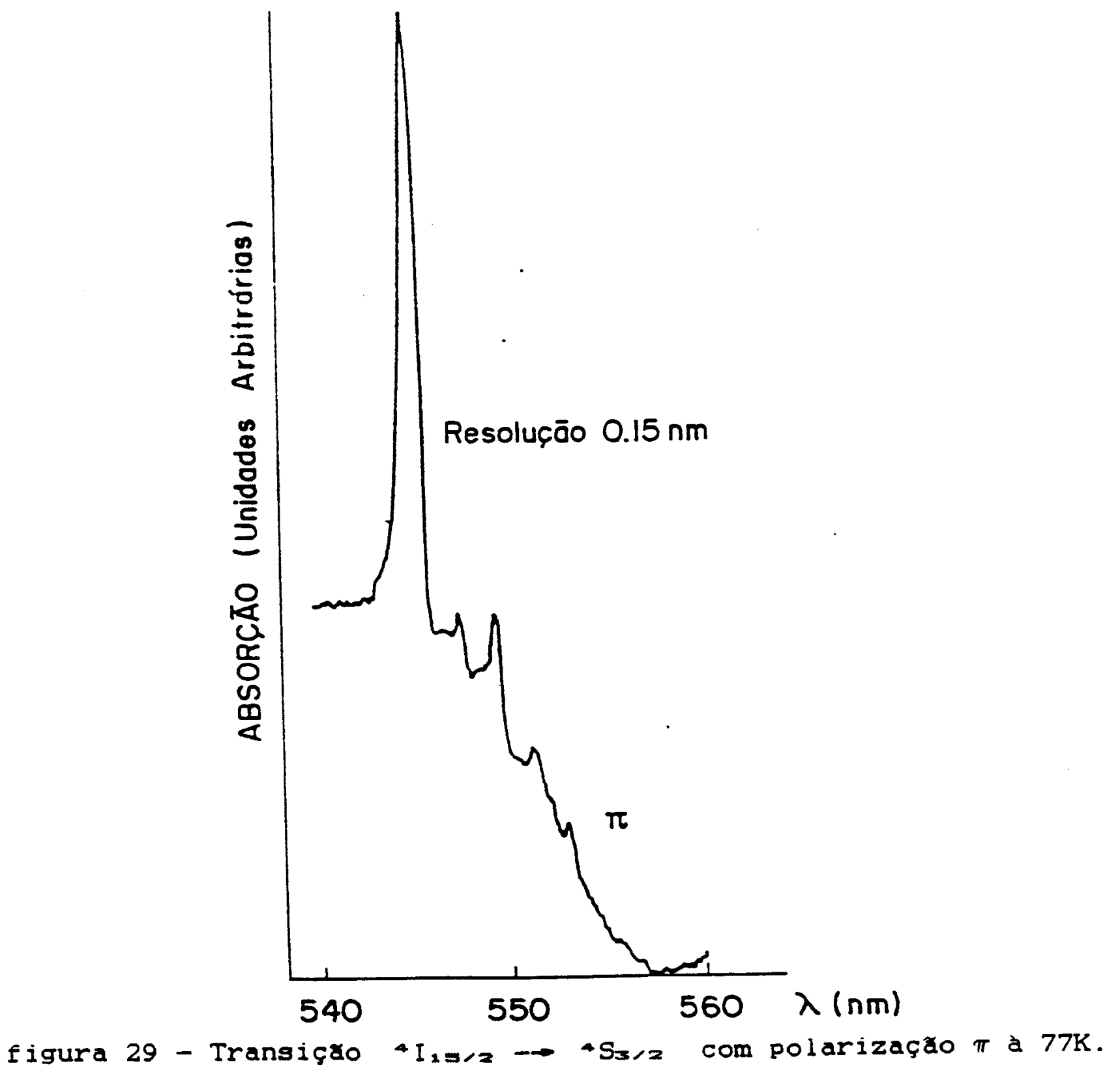




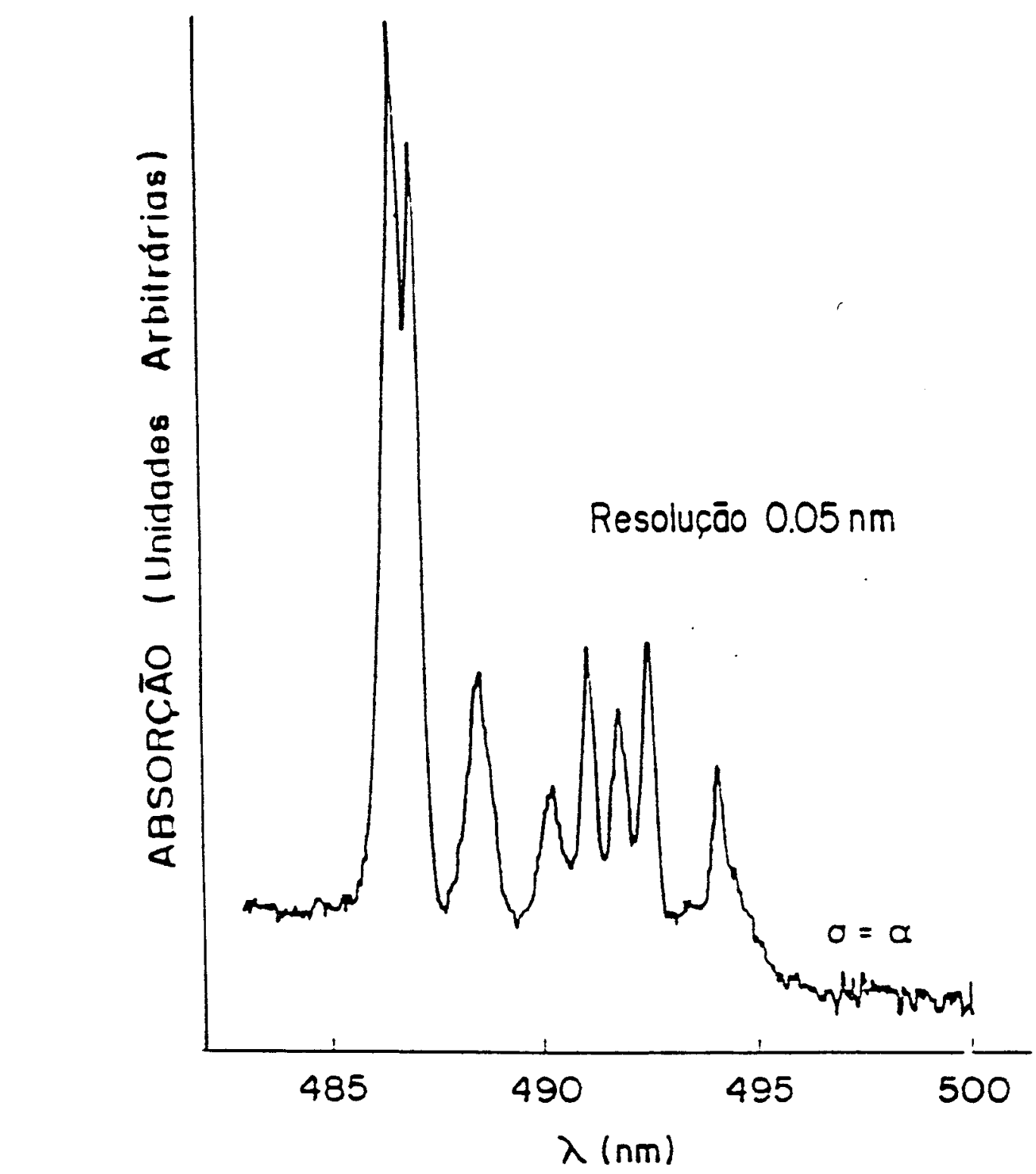

figura 32 - Transiçå $\mathrm{I}_{1=/ 2}-{ }^{4} \mathrm{~F}_{7 / 2}$ com polarizaçăo $\sigma \propto$ a $77 \mathrm{~K}$.

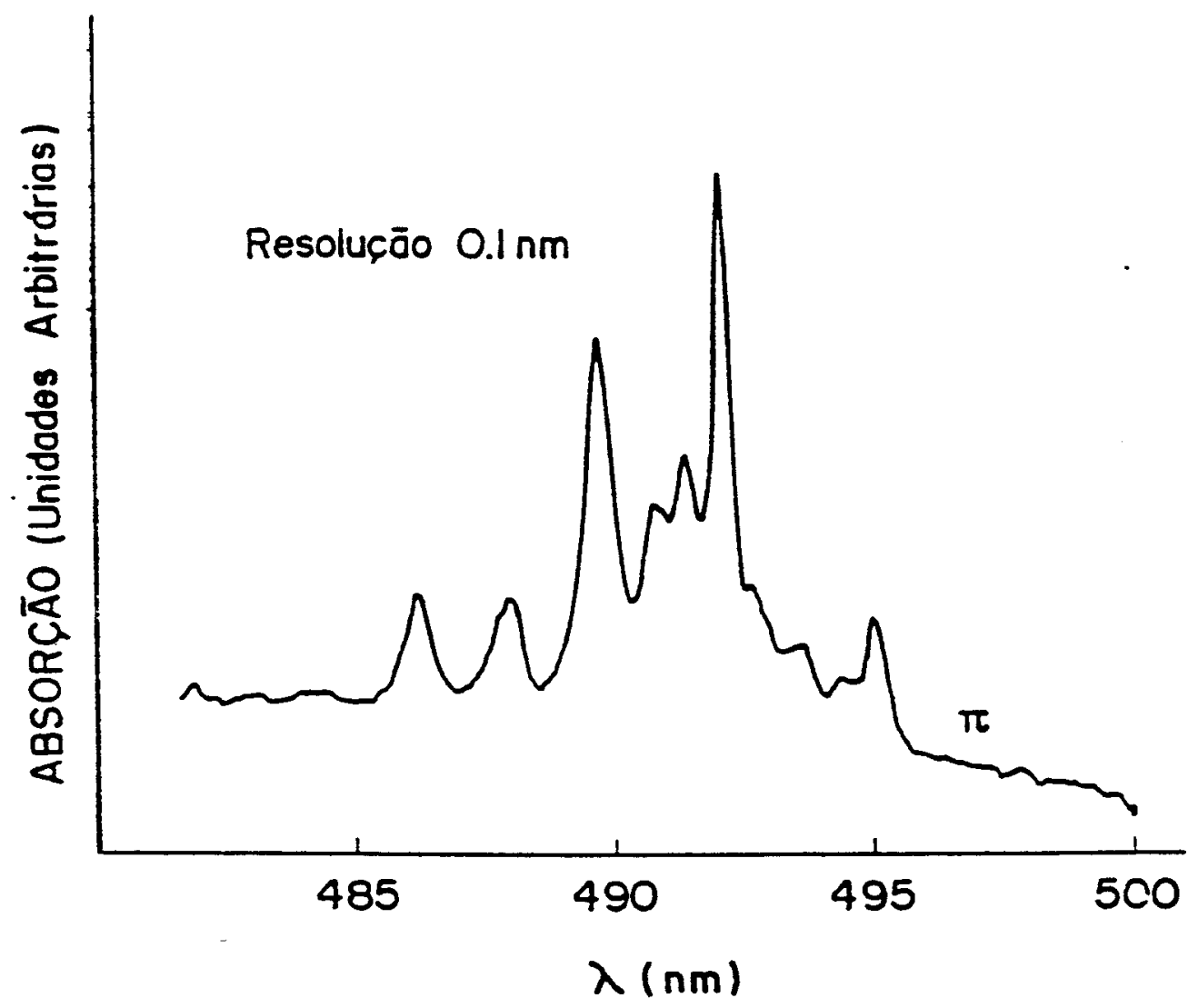

figura 33 - Transiçăo $4 I_{15 / 2}-{ }^{4} F_{7 / 2}$ com polarizaçăo $\pi$ à $77 \mathrm{~K}$. 


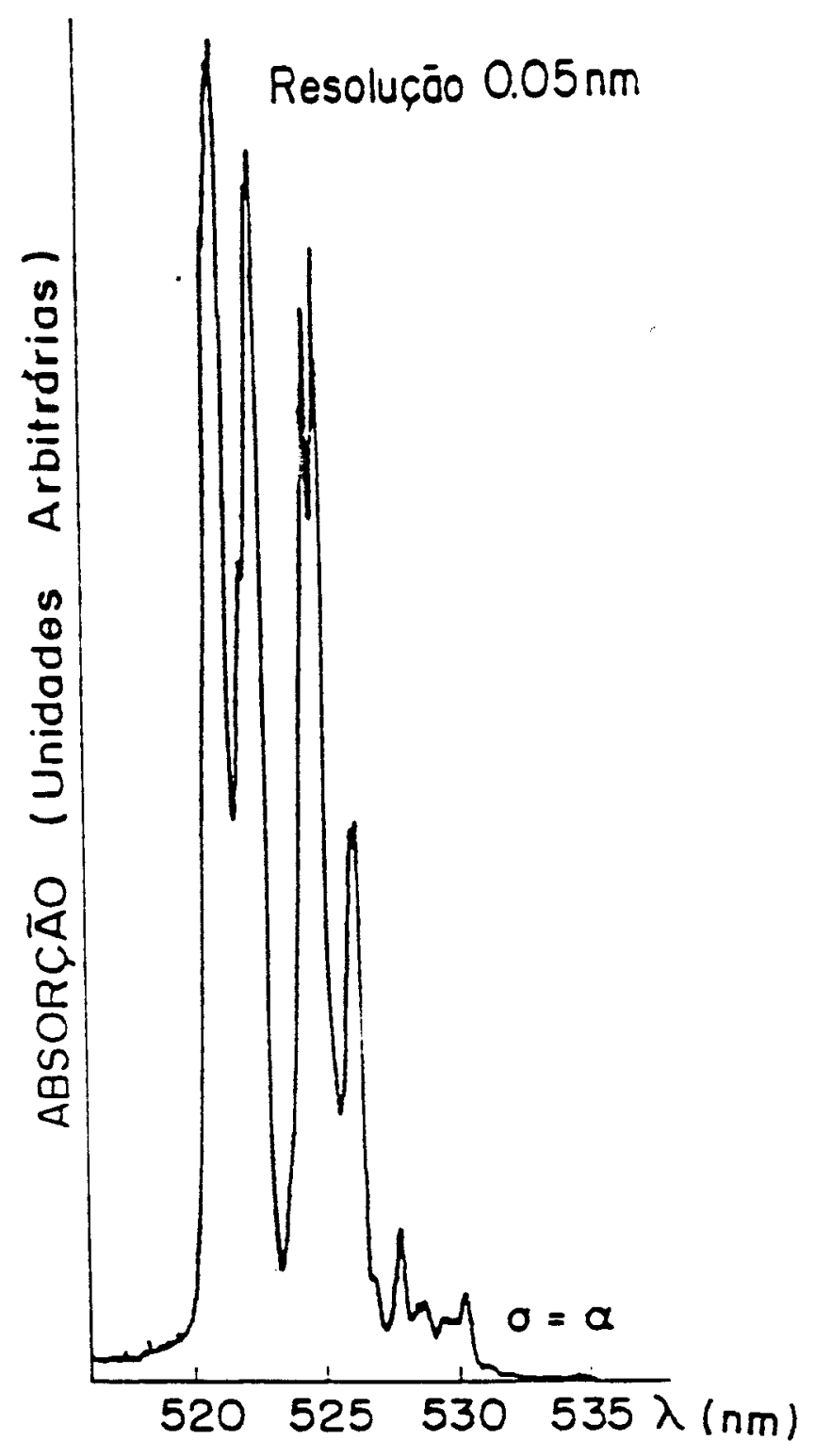

figura 30 - Transiçå ${ }^{4} \mathrm{I}_{1=/ 2} \rightarrow \mathrm{ZH}_{11,2}$ com polarizaçao $\sigma \alpha$ a $77 \mathrm{~K}$.

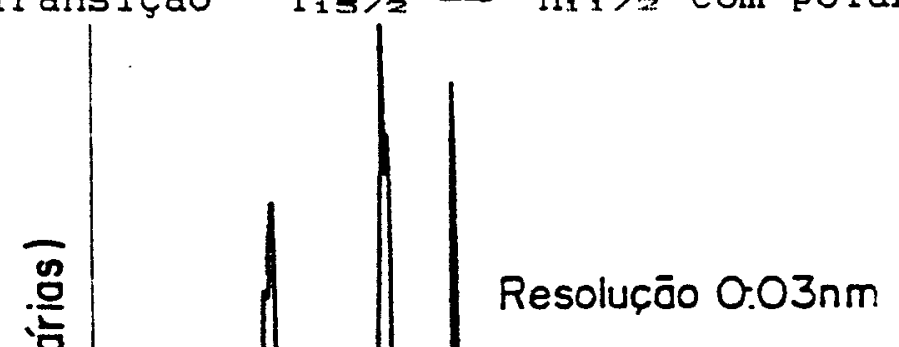

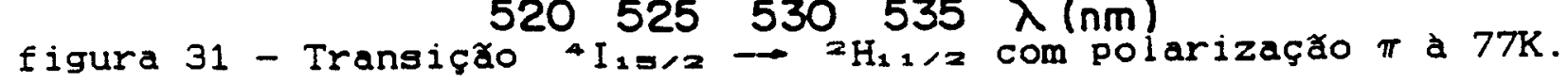




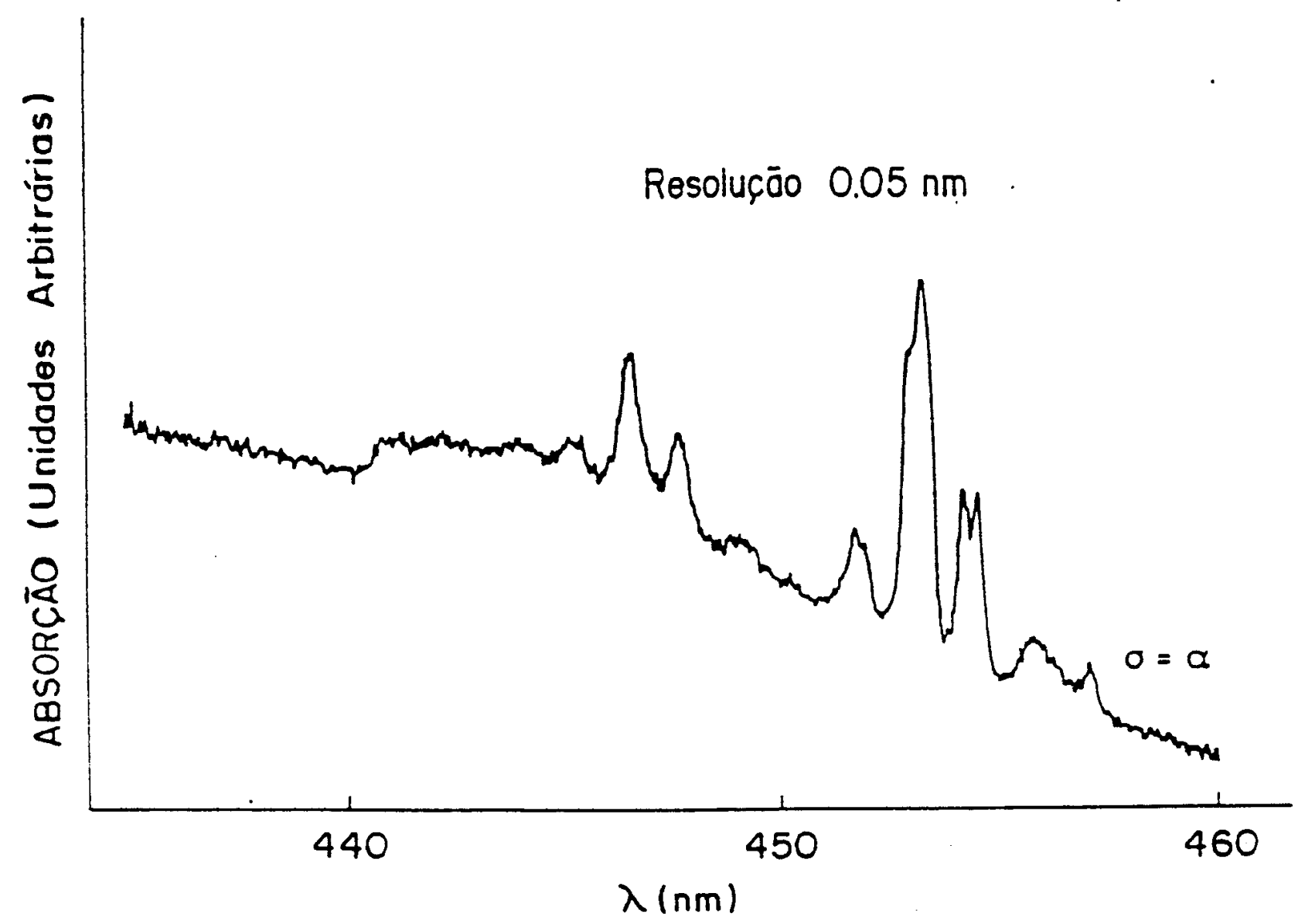

$\begin{aligned} \text { figura } 34- & \text { Transiçסes }{ }^{4} I_{1=/ z}=-{ }^{4} \mathrm{~F}=/ 2 \text { e }{ }^{4} I_{1=/ z-\rightarrow}{ }^{4} \mathrm{~F}=1 z \text { com } \\ & \text { polarização } \sigma \propto \mathrm{a} 77 \mathrm{~K} .\end{aligned}$

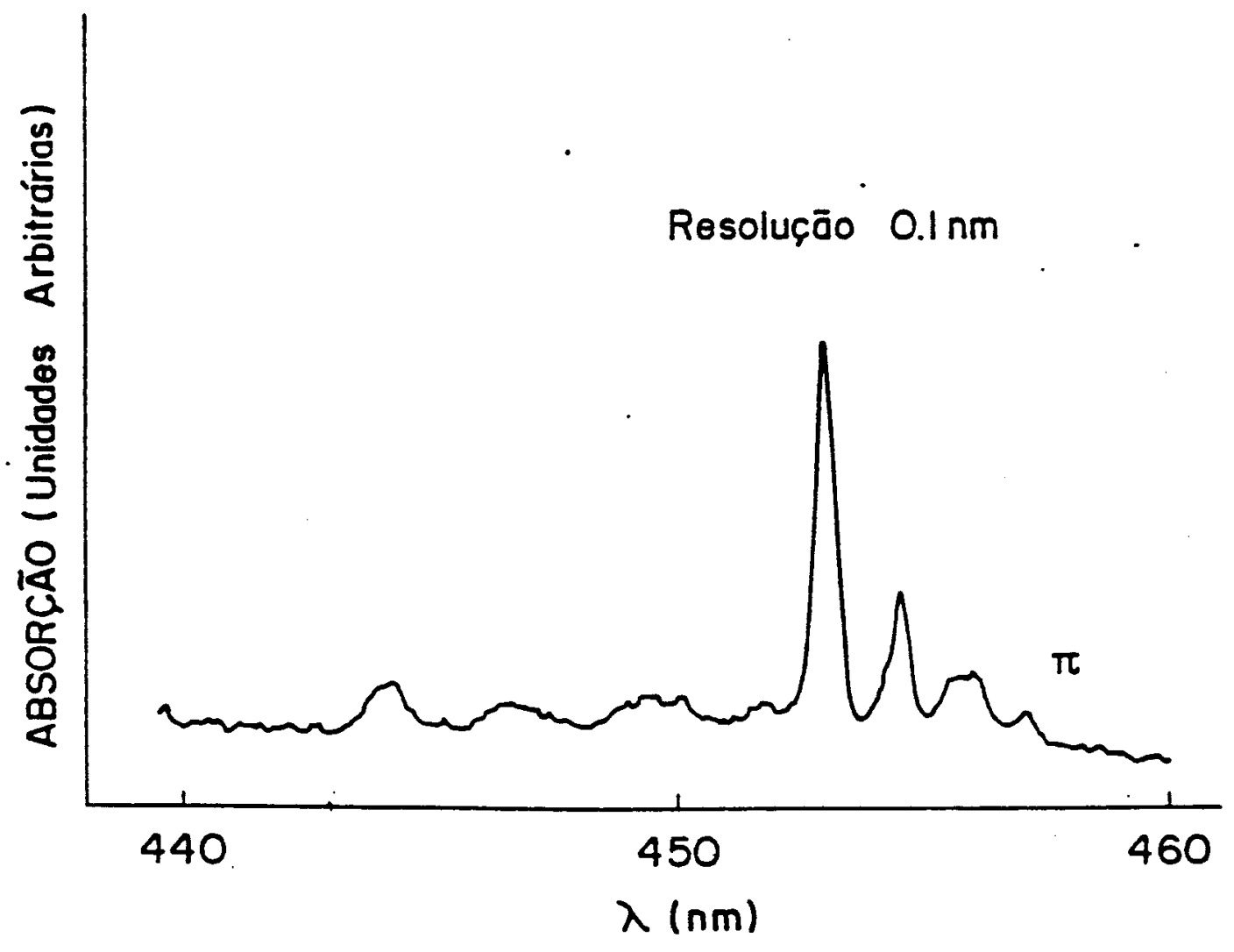

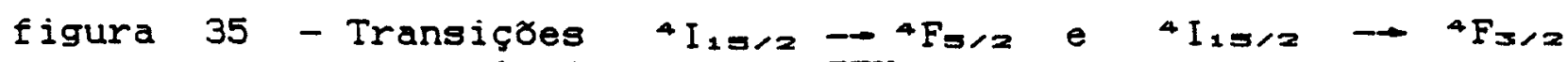
com polarizaçăo $\pi$ a $77 \mathrm{~K}$. 


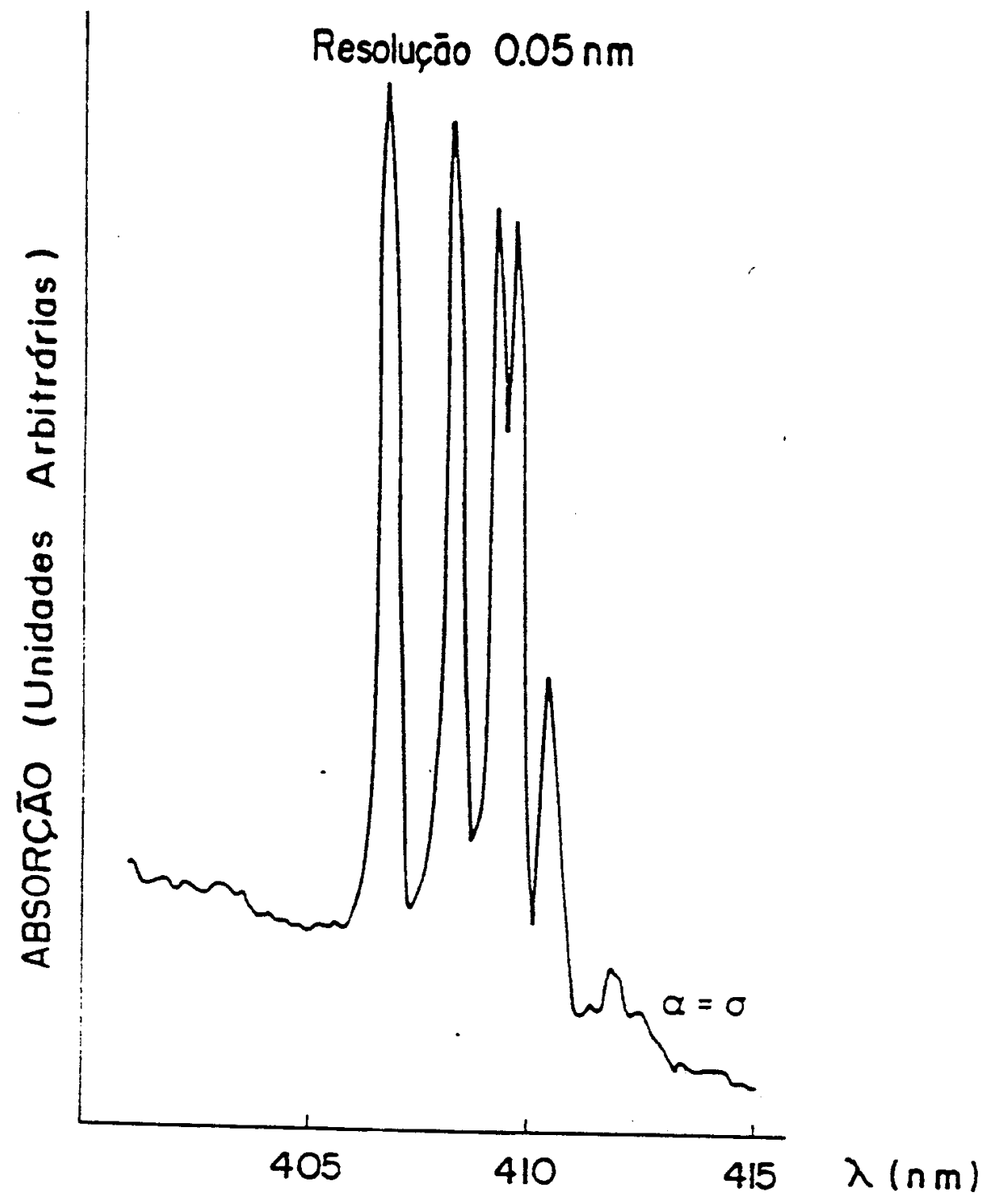

figura 36 - Transição $4 \mathrm{I}_{13 / 2}-\mathrm{F}_{\infty}=$ com polarizaçăo $\sigma \alpha$ a $77 \mathrm{~K}$.

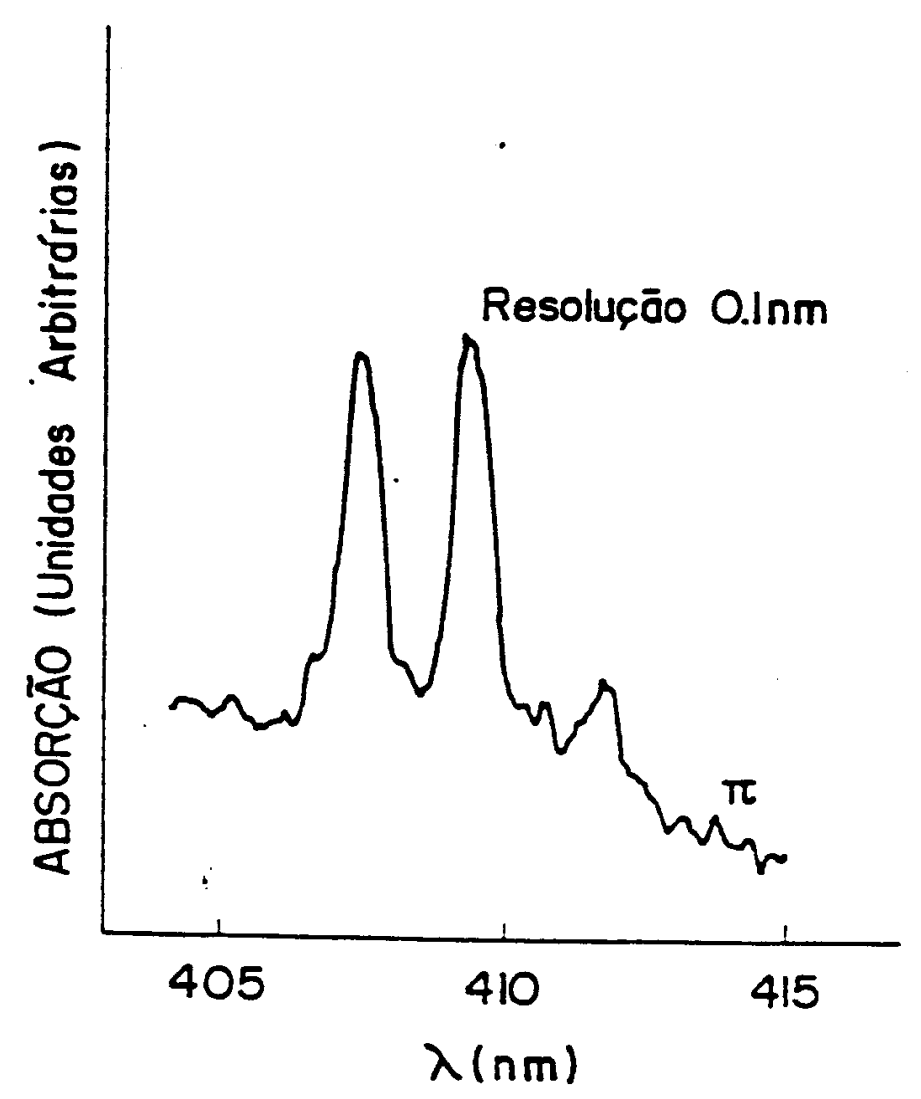




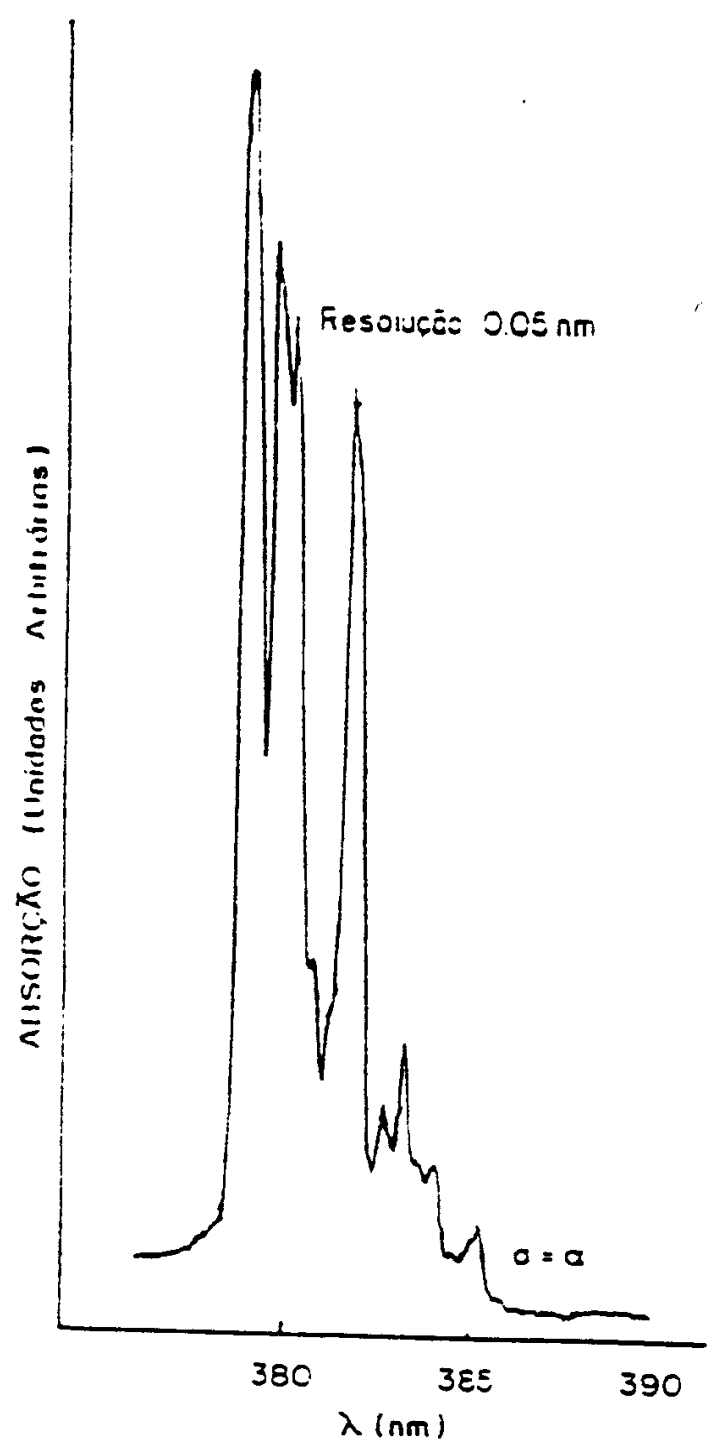

66

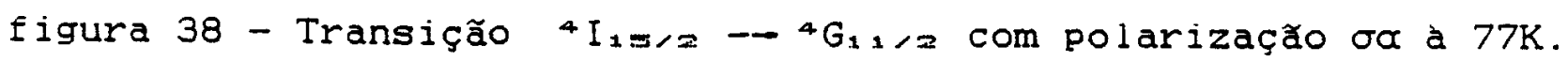

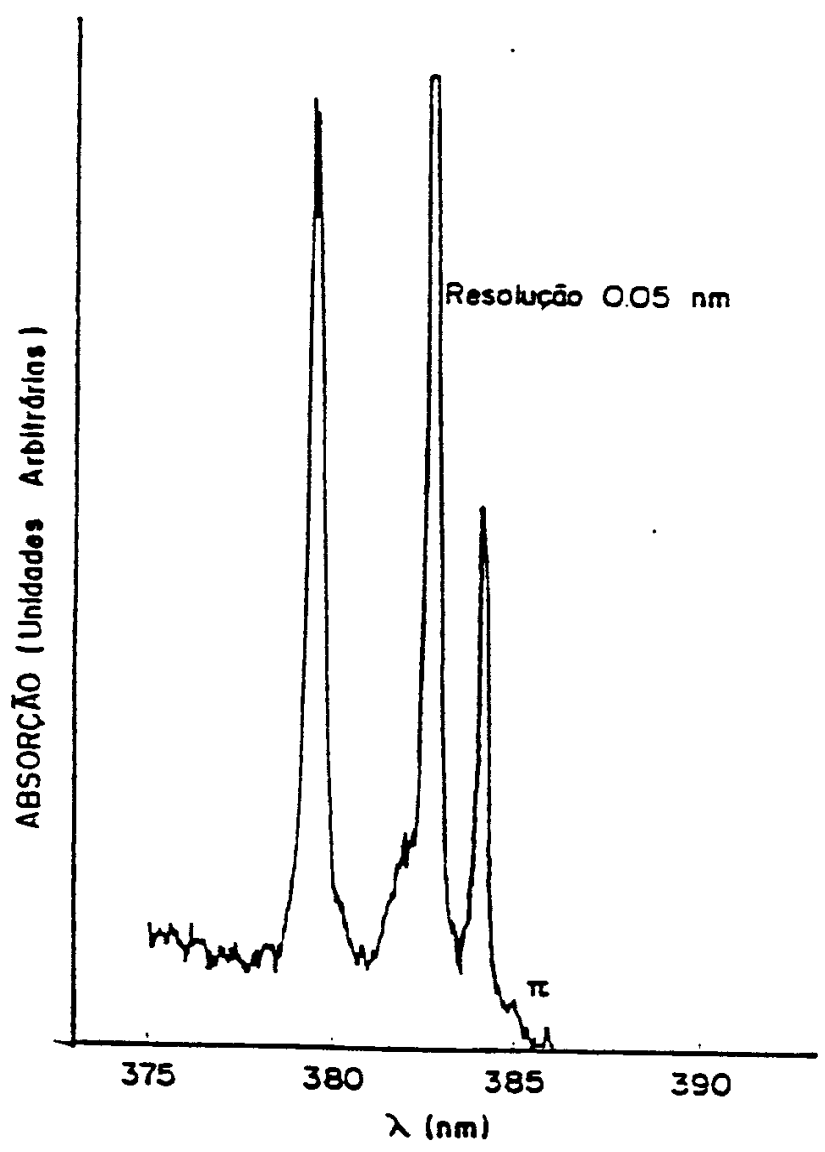

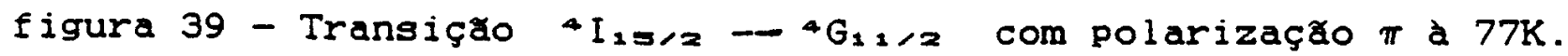


Nas tabelas a seguir temos os comprimentos de onda relativos a cada transição mostrada anteriormente.

TABELA 7 - Transição ${ }^{4} I_{13 / 2} \rightarrow{ }^{4} I_{13 / 2}$ do Er+3: LiNbO=3 a $77 \mathrm{~K}$.

\begin{tabular}{lll}
\hline POLARIZAÇO. & $\lambda(\mathrm{nm})$ & ENERGIA $\left(\mathrm{cm}^{-1}\right)$ \\
\hline 1561.76 & 6404.31 \\
1546.18 & 6467.57 \\
1531.76 & 6528.42 \\
1517.94 & 6587.87 \\
1508.24 & 6630.27 \\
1505.88 & 6640.62 \\
1482.94 & 6743.36 \\
1474.41 & 6782.37 \\
1469.41 & 6805.00 \\
1480.00 & 6756.76 \\
1489.71 & 6712.73 \\
\hline
\end{tabular}


TABELA 8 - Transiçăo ${ }^{4} I_{11 / 2 \rightarrow}{ }^{4} I_{1} / 2$ do Er+3:LiNbos a $77 \mathrm{~K}$.

\begin{tabular}{ccc}
\hline POLARIZAÇO. & $\lambda(\mathrm{nm})$ & ENERGIA $\left(\mathrm{cm}^{-1}\right)$ \\
\hline 969.76 & 10311.78 \\
971.20 & 10296.54 \\
974.35 & 10263.22 \\
975.36 & 10252.58 \\
& 980.47 & 10199.18 \\
& 982.00 & 10183.30 \\
& 960.00 & 10141.99 \\
& 990.00 & 10101.01 \\
& 990.90 & 10091.84 \\
\hline
\end{tabular}


TABELA 9 - Transiçăo ${ }^{4} I_{1=/ 2} \rightarrow{ }^{4} I_{\theta / 2}$ do Er+3 ${ }^{+i N i N b O}=$ a $77 \mathrm{~K}$.

\begin{tabular}{|c|c|c|}
\hline POLARIZAÇAO & $\lambda(\mathrm{nm})$ & ENERGIA $\left(\mathrm{cm}^{-1}\right)$ \\
\hline \multirow{12}{*}{$\sigma$} & 816,8 & 12242.9 \\
\hline & 812,2 & 12312,2 \\
\hline & 810.8 & 12333.5 \\
\hline & 808.7 & 12365.5 \\
\hline & 808.0 & 12376.2 \\
\hline & 806.7 & 12396.2 \\
\hline & 804.0 & 12437.8 \\
\hline & 802.0 & 12468,8 \\
\hline & 800.7 & 12489.1 \\
\hline & 798.0 & 12531,3 \\
\hline & 794.7 & 12583.4 \\
\hline & 794.0 & 12594.5 \\
\hline \multirow{4}{*}{$\pi$} & 807.6 & 12382.4 \\
\hline & 805.7 & 12411.6 \\
\hline & 803.0 & 12453.3 \\
\hline & 796.9 & 12548.6 \\
\hline
\end{tabular}




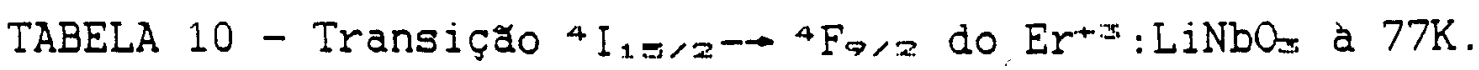

\begin{tabular}{|c|c|c|}
\hline POLARIZAÇAO & $\lambda(\mathrm{nm})$ & ENERGIA $\left(\mathrm{cm}^{-1}\right)$ \\
\hline \multirow{14}{*}{$\sigma$} & 666.6 & 15001.5 \\
\hline & 665.7 & 15021.8 \\
\hline & 663.1 & 15080.7 \\
\hline & 662.2 & 15101,2 \\
\hline & 660.4 & 15142,3 \\
\hline & 659.6 & 15160.7 \\
\hline & 657.7 & 15204.5 \\
\hline & 655.5 & 15255.5 \\
\hline & 655.0 & 15267.2 \\
\hline & 654.4 & 15281.2 \\
\hline & 654.2 & 15285.8 \\
\hline & 651.9 & 15339.8 \\
\hline & 651.4 & 15351,6 \\
\hline & 650.5 & 15372,8 \\
\hline \multirow{11}{*}{$\pi$} & 664,5 & 15046.6 \\
\hline & 663.1 & 15078,4 \\
\hline & 662.2 & 15101.2 \\
\hline & 660.3 & 15142.3 \\
\hline & 659.5 & 15160,7 \\
\hline & 657.7 & 15204,5 \\
\hline & 657,1 & 15216.1 \\
\hline & 655.7 & 15248.6 \\
\hline & 654.2 & 15283.5 \\
\hline & 651.1 & 15358,6 \\
\hline & 650.5 & 15370.4 \\
\hline
\end{tabular}




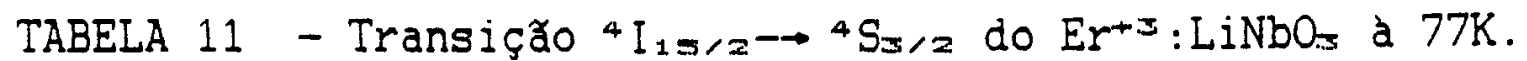

\begin{tabular}{|c|c|c|}
\hline POLARIZAÇAO & $\lambda(\mathrm{nm})$ & ENERGIA $\left(\mathrm{cm}^{-1}\right)$ \\
\hline \multirow{5}{*}{$\sigma$} & $\Rightarrow \quad 551.8$ & 18122.5 \\
\hline & 549,7 & 18191,7 \\
\hline & $=547.9$ & $18251.5-*$ \\
\hline & $\therefore \quad 547.1$ & 18278.2 \\
\hline & 545.2 & $18341.9 \ldots$ \\
\hline \multirow{7}{*}{$\pi$} & (1) 553.2 & 18076.6 \\
\hline & 552.3 & 18106,1 \\
\hline & $\because 551.5$ & 18132.4 \\
\hline & $-\quad 549.7$ & 18191.7 \\
\hline & 547.7 & 18258.2 \\
\hline & $547: 0$ & 18281,5 \\
\hline & $\therefore \quad 545.2$ & $18341.9 . x$ \\
\hline
\end{tabular}
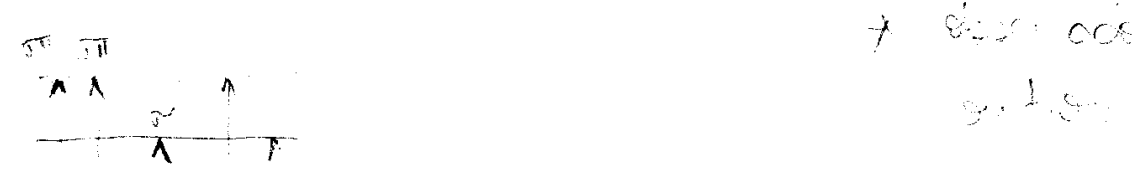
TABELA 12 - Transiçao ${ }^{4} \mathrm{I}_{1=/ 2-\rightarrow}{ }^{4} \mathrm{H}_{11 / 2}$ do Er+3:LiNbO= a $77 \mathrm{~K}$.

\begin{tabular}{|c|c|c|}
\hline POLARIZAÇAOO & $\lambda(\mathrm{nm})$ & ENERGIA $\left(\mathrm{cm}^{-1}\right)$ \\
\hline \multirow{15}{*}{$\sigma$} & 530,3 & 18857.3 \\
\hline & 529.5 & 18885.7 \\
\hline & 528.6 & 18917.9 \\
\hline & 527.8 & 18975.3 \\
\hline & 526,9 & 18978.9 \\
\hline & 526.3 & 19000.6 \\
\hline & 526.1 & 19007.8 \\
\hline & 524.9 & 19051,2 \\
\hline & 524,5 & 19065,8 \\
\hline & 522,6 & 19135.1 \\
\hline & 522.4 & 19142.4 \\
\hline & 522.1 & 19153,4 \\
\hline & 521.2 & 19186.5 \\
\hline & 521,0 & 19193,9 \\
\hline & 520,8 & 19201.2 \\
\hline \multirow{10}{*}{$\pi$} & 531.4 & 18818,2 \\
\hline & 529.5 & 18885.7 \\
\hline & 527.9 & 18943.0 \\
\hline & 526,4 & 18997.0 \\
\hline & 525.5 & 19019.5 \\
\hline & 525,3 & 19036.7 \\
\hline & 525.1 & 19044,0 \\
\hline & 524.7 & 19058.5 \\
\hline & 521,4 & 19179.1 \\
\hline & 521.0 & 19193.9 \\
\hline
\end{tabular}

490,8

489,8

488,0

486,3
20374.9

20416,5

20491,8

20563,4 
TABELA 13 - Transiçå ${ }^{4} \mathrm{I}_{1=/ 2}{ }^{4}{ }^{4} \mathrm{~F}_{7 / 2}$ do Er+3:LiNbO= à $77 \mathrm{~K}$.

\begin{tabular}{|c|c|c|}
\hline POLARIZAÇAO & $\lambda(\mathrm{nm})$ & ENERGIA $\left(\mathrm{cm}^{-1}\right)$ \\
\hline \multirow{8}{*}{$\sigma$} & 494.1 & 20238.8 \\
\hline & 492.5 & 20304.6 \\
\hline & 491.8 & 20333,5 \\
\hline & 491,1 & 20362.5 \\
\hline & 490,2 & 20399.8 \\
\hline & 488.6 & 20466,6 \\
\hline & 487.0 & 20533.9 \\
\hline & 486.6 & 20550,8 \\
\hline \multirow{11}{*}{$\pi$} & 495.1 & 20197,9 \\
\hline & 494.4 & 20226.5 \\
\hline & 493.6 & 20259.3 \\
\hline & 493.0 & 20284.0 \\
\hline & 492.7 & 20296.3 \\
\hline & 492.1 & 20321.1 \\
\hline & 491,4 & 20350,0 \\
\hline & 490.8 & 20374.9 \\
\hline & 489.8 & 20416.5 \\
\hline & 488.0 & 20491,8 \\
\hline & 486,3 & 20563,4 \\
\hline
\end{tabular}




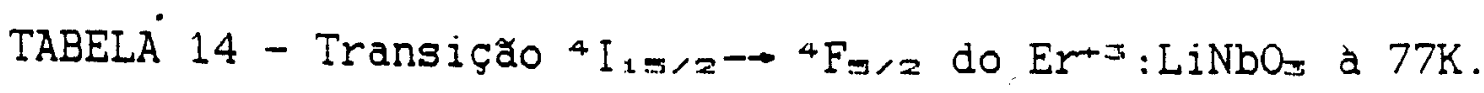

\begin{tabular}{cll}
\hline POLARIZAÇO & $\lambda(\mathrm{nm})$ & ENERGIA $\left(\mathrm{cm}^{-1}\right)$ \\
\hline & 456,1 & 21925,0 \\
& 455,8 & 21939,4 \\
& 454,6 & 21997,4 \\
& 454,2 & 22016,7 \\
& 453,4 & 22055,6 \\
& 453,0 & 22075,1 \\
& 451,7 & 22138,6 \\
\hline & 457,1 & 21877,1 \\
& 456,0 & 21929,8 \\
& 455,7 & 21944,3 \\
& 454,6 & 21997,4 \\
& 453.1 & 22070.2 \\
\hline
\end{tabular}

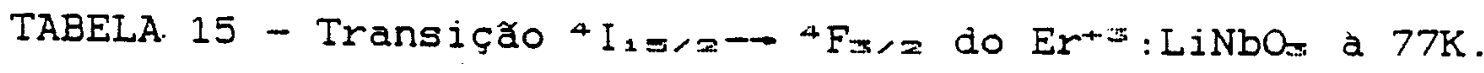

\begin{tabular}{ccc}
\hline POLARIZAÇAO & $\lambda(\mathrm{nm})$ & ENERGIA $\left(\mathrm{cm}^{-1}\right)$ \\
\hline$\sigma$ & 447.7 & 22336.4 \\
& 446.6 & 22391.4 \\
& 445.2 & 22461.8 \\
\hline$\pi$ & 449.5 & 22246.9 \\
& 446.5 & 22396.4 \\
& 444.0 & 22522.5 \\
\hline
\end{tabular}




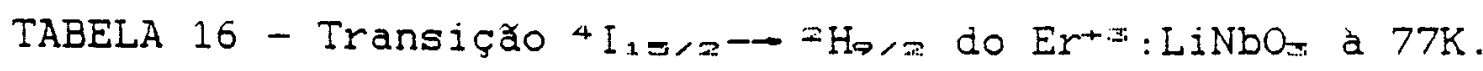

\begin{tabular}{ccc}
\hline POLARIZAÇAO & $\lambda(\mathrm{nm})$ & ENERGIA $\left(\mathrm{cm}^{-1}\right)$ \\
\hline & 412,5 & 24242,4 \\
& 411.8 & 24283,6 \\
& 411.4 & 24307,2 \\
& 410.5 & 24360,5 \\
& 409.7 & 24408,1 \\
& 409.3 & 24432,0 \\
& 408.3 & 24491,8 \\
& 406.8 & 24582.1 \\
\hline & 411.8 & 24283,6 \\
& 409.4 & 24426,0 \\
& 407.5 & 24539.9 \\
\hline
\end{tabular}




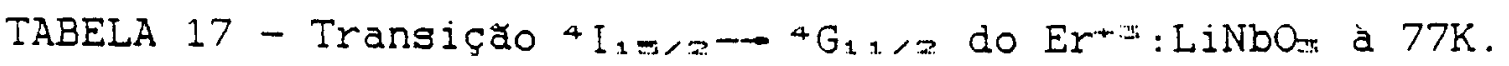

\begin{tabular}{|c|c|c|}
\hline POLARIZAÇO & $\lambda(\mathrm{nm})$ & ENERGIA $\left(\mathrm{cm}^{-1}\right)$ \\
\hline \multirow{11}{*}{$\sigma$} & 385.3 & 25953.8 \\
\hline & 384,5 & 26007.8 \\
\hline & 384.0 & 26041.7 \\
\hline & 386.6 & 25866,5 \\
\hline & 386.2 & 25893,3 \\
\hline & 385.7 & 25926.9 \\
\hline & 381.8 & 26191.7 \\
\hline & 380.8 & 26260,5 \\
\hline & 380.2 & 26301.9 \\
\hline & 379.8 & 26329.6 \\
\hline & 379.1 & 26378.3 \\
\hline \multirow{4}{*}{$\pi$} & 384.1 & 26034.9 \\
\hline & 382.7 & 26130,1 \\
\hline & 379.6 & 26343.5 \\
\hline & 379.4 & 26357,4 \\
\hline
\end{tabular}

\subsection{EMISSAO POLARIZADA}

As medidas de emissao polarizada foram feitas a temperatura ambiente e de nitrogennio liquido (77K). Nas iguras 40 a 53 temos os resultados obtidos. Nas tabelas 18 a 21 mostramos os comprimentos de onda e as energias de todas transições medidas. 


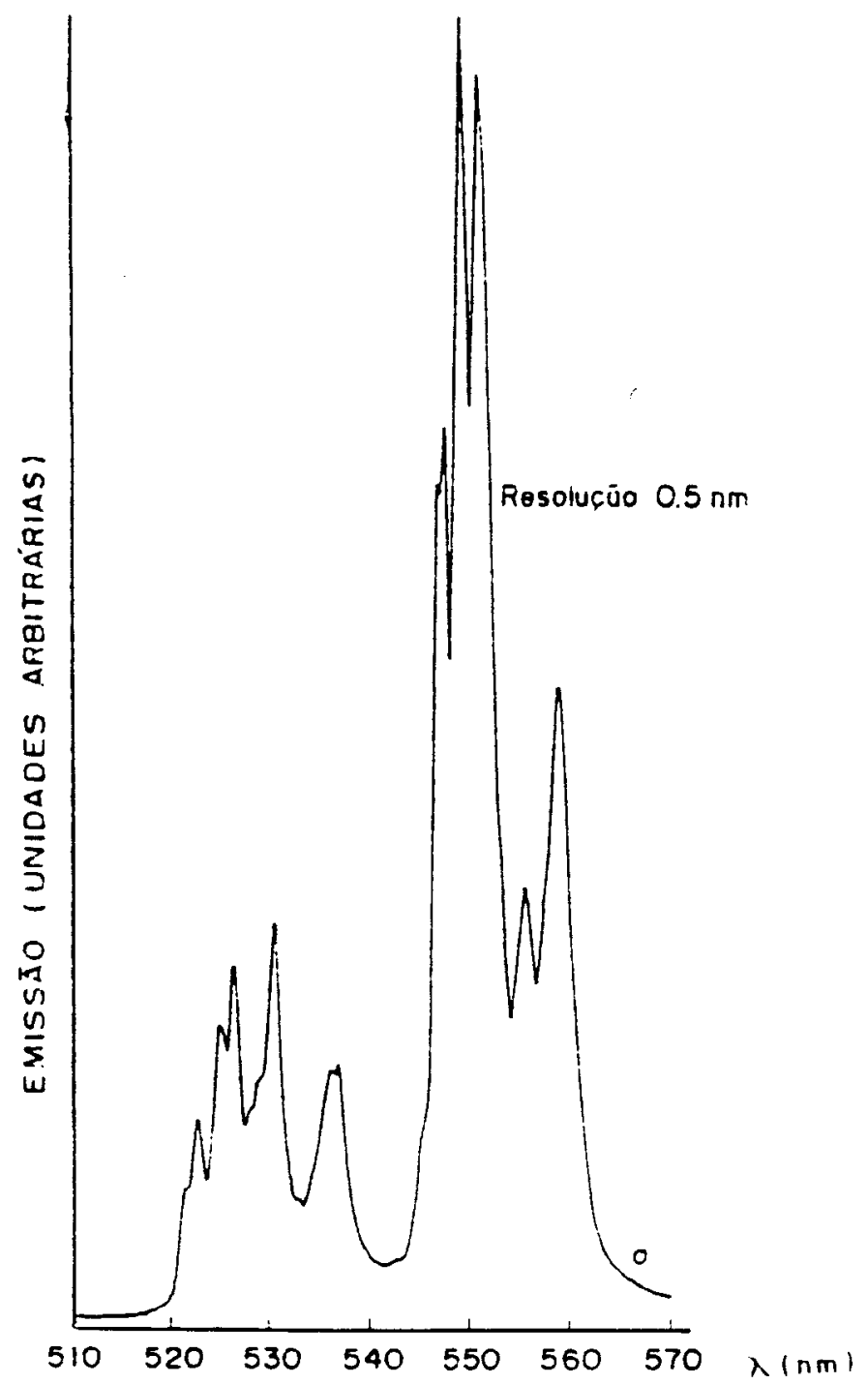

figura 40 - Espectro de emissão do $\mathrm{LiNbO}_{\mathbf{3}}$ :Er+s relativo as transiçoes ${ }^{2} \mathrm{H}_{11 / 2} \rightarrow{ }^{4} \mathrm{I}_{1 \leq / 2}$ e ${ }^{4} \mathrm{~S}_{3 / 2} \rightarrow{ }^{4} \mathrm{I}_{1=1 / 2}$ com polarizaçăo $\sigma$ à temperatura ambiente.

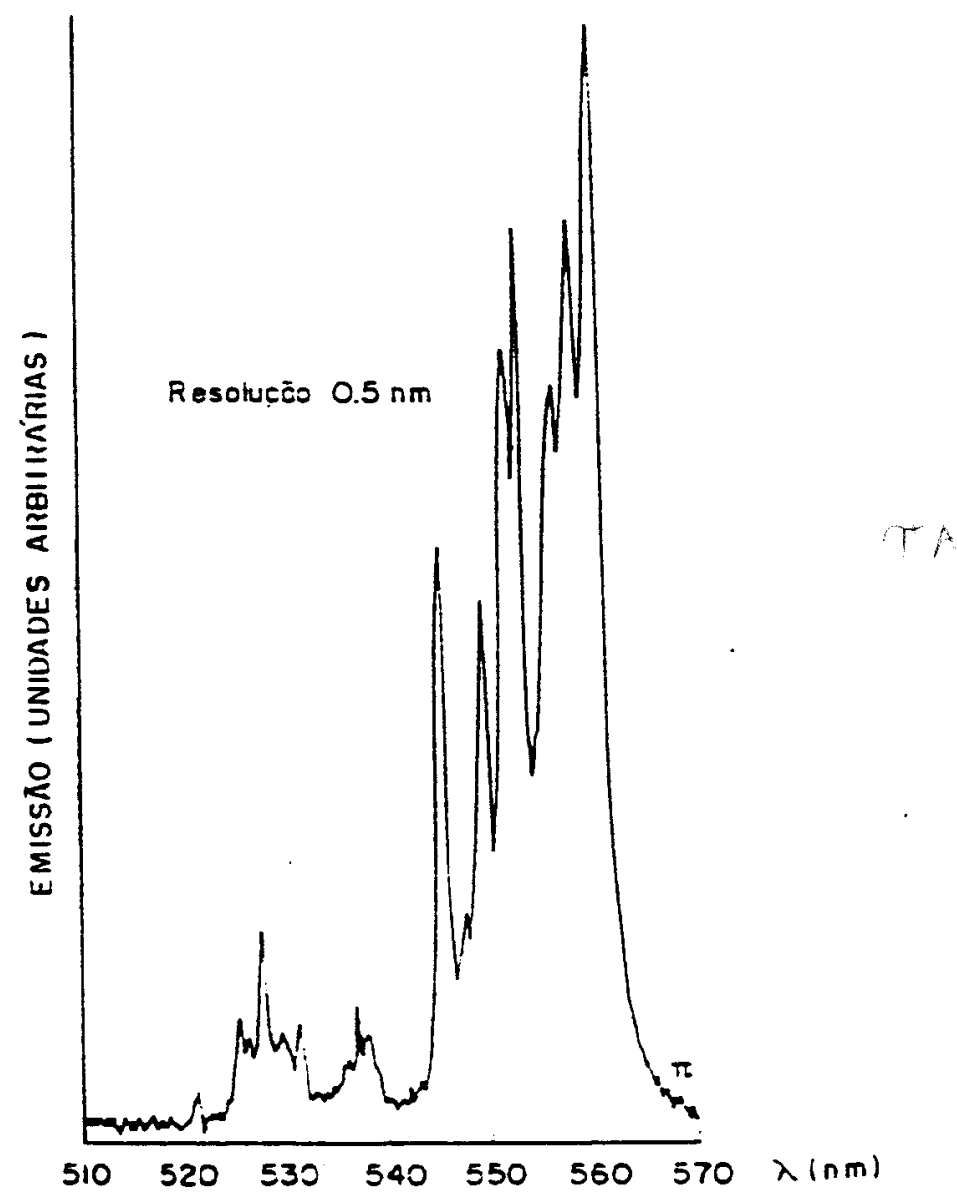

figura 41 - Espectro de emissao do LiNbOs:Erm relativo as transiçбes ${ }^{2} \mathrm{H}_{11 / 2} \rightarrow{ }^{4} \mathrm{I}_{15 / 2}$ e ${ }^{4} \mathrm{~S}_{3 / 2}-{ }^{4} \mathrm{I}_{13 / 2}$ com polarizaçao $\pi$ a temperatura ambiente. 


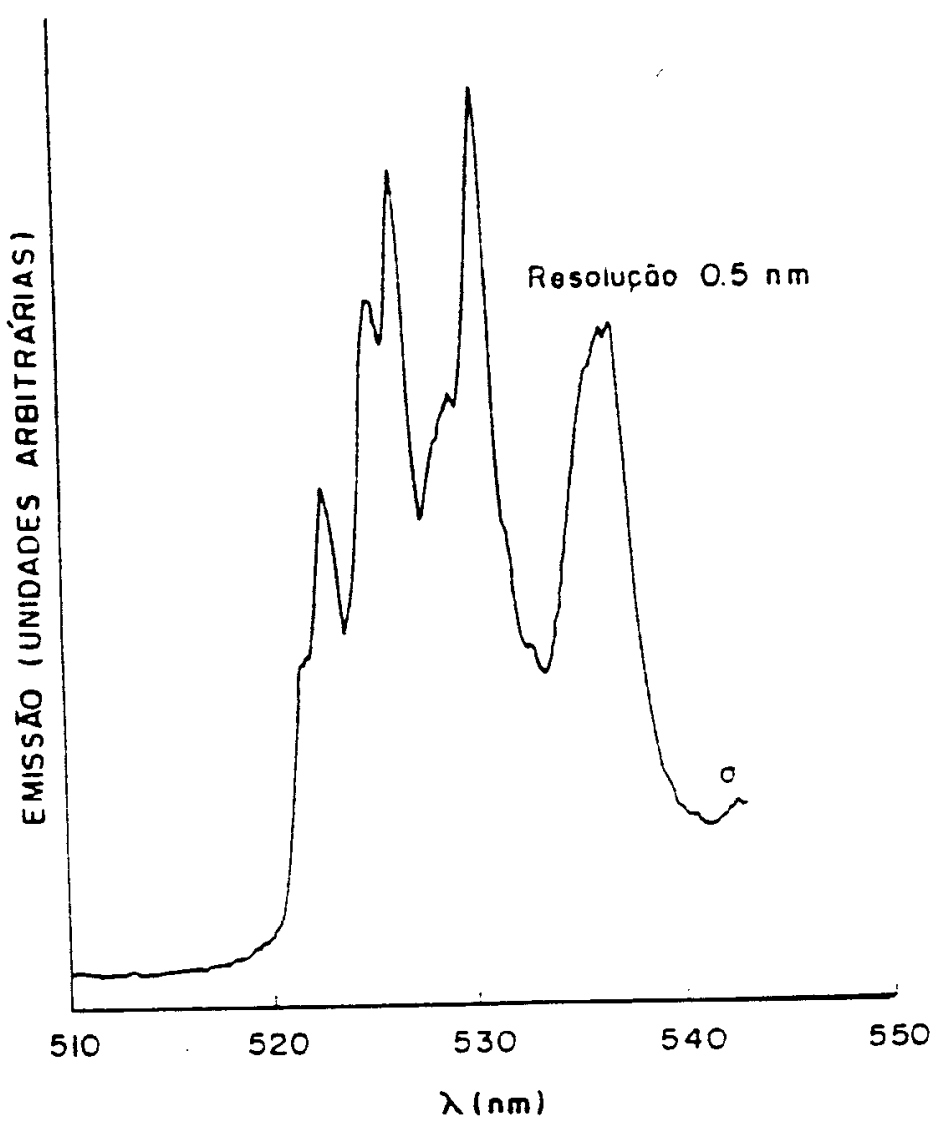

figura 42 - Espectro de emissão do LiNbos:Er+3 relativo a transição ${ }^{2} H_{11,2} \rightarrow{ }^{4} I_{1=/ 2}$ com polarizaçăo $\sigma$ a temperatura ambiente.

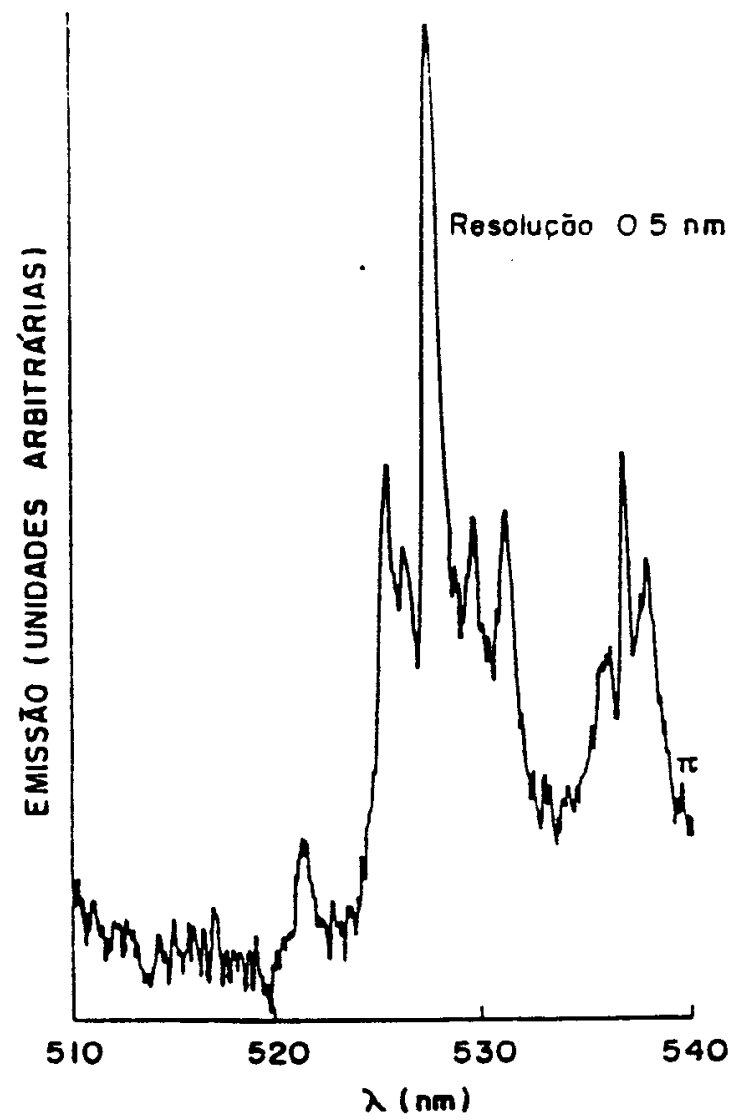

figura 43 - Espectro de emissto do LiNbos:Er= relativo a transiçăo ${ }^{2} \mathrm{H}_{11 / 2}-{ }^{4} \mathrm{I}_{13 / 2} \operatorname{com}$ polarizaçao $\pi$ à temperatura ambiente. 


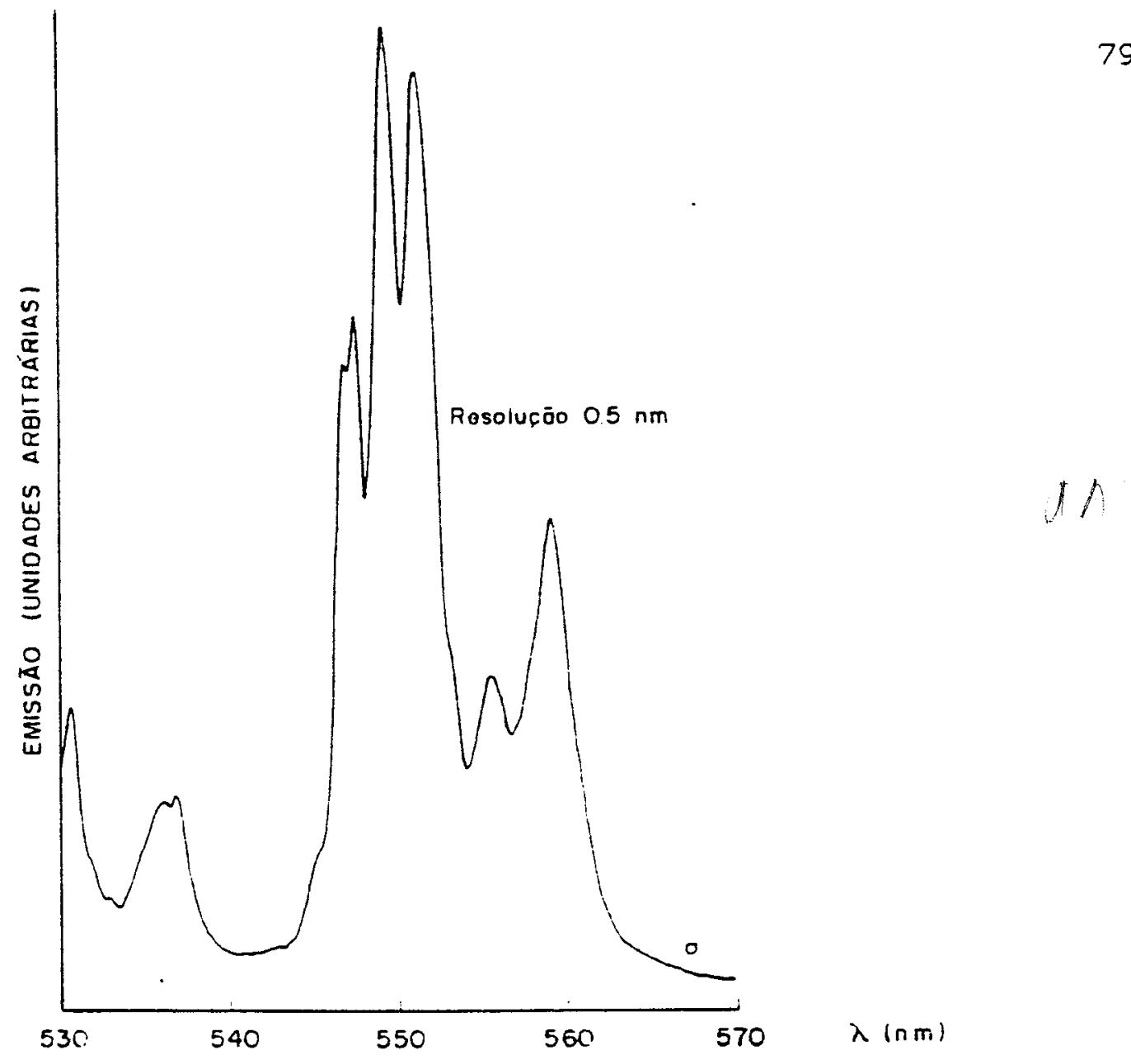

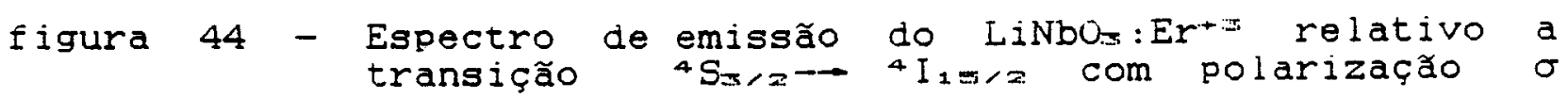
a temperatura ambiente.

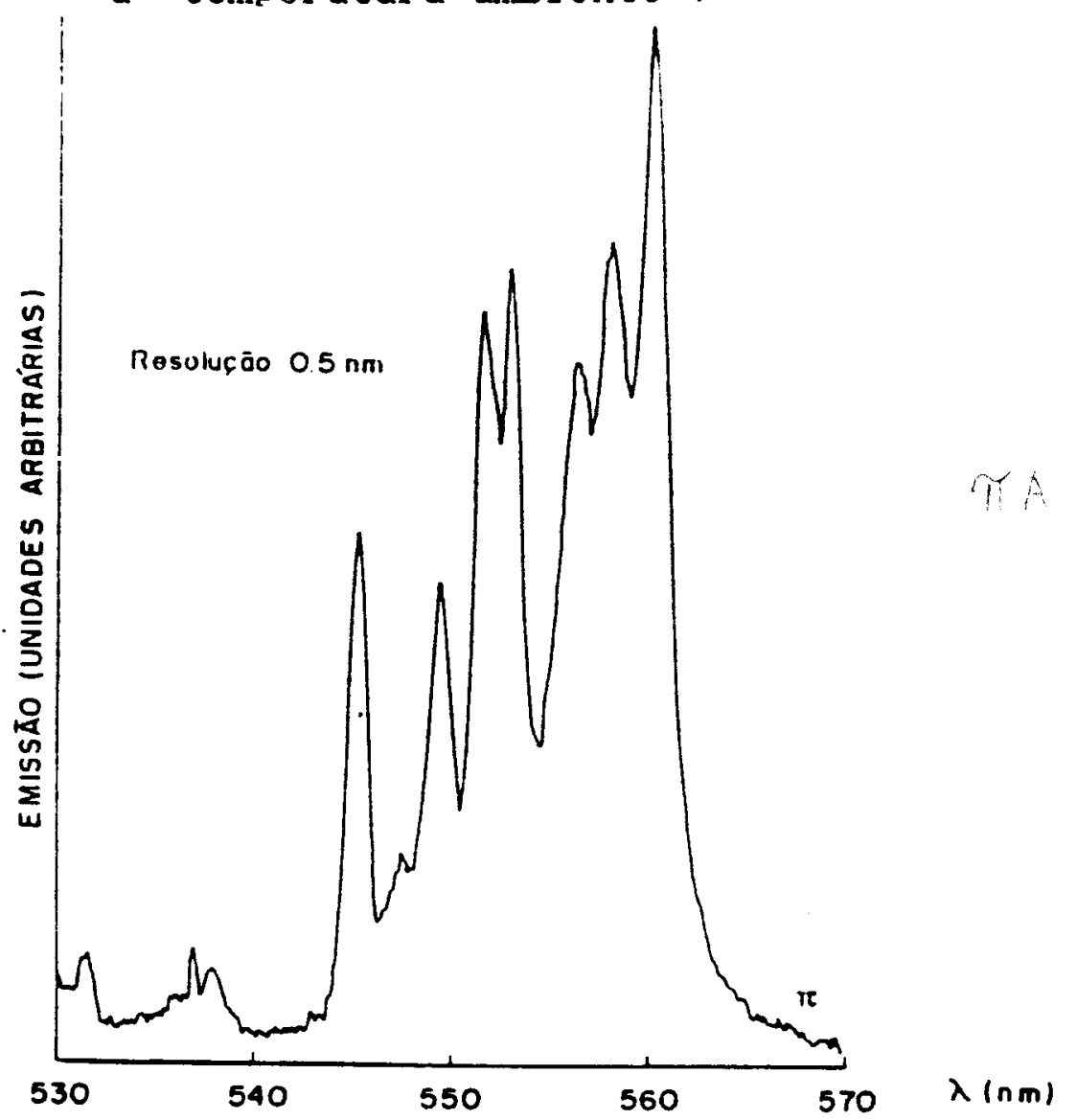

figura 45 - Espectro de emissao do $\mathrm{LiNbO}_{\mathrm{s}}: \mathrm{Er}^{+3}$ relativo a transiçăo ${ }^{4} \mathrm{~S}_{3 / 2} \rightarrow{ }^{4} \mathrm{I}_{13 / 2}$ com polarizaçăo $\pi$ à temperatura ambiente. 


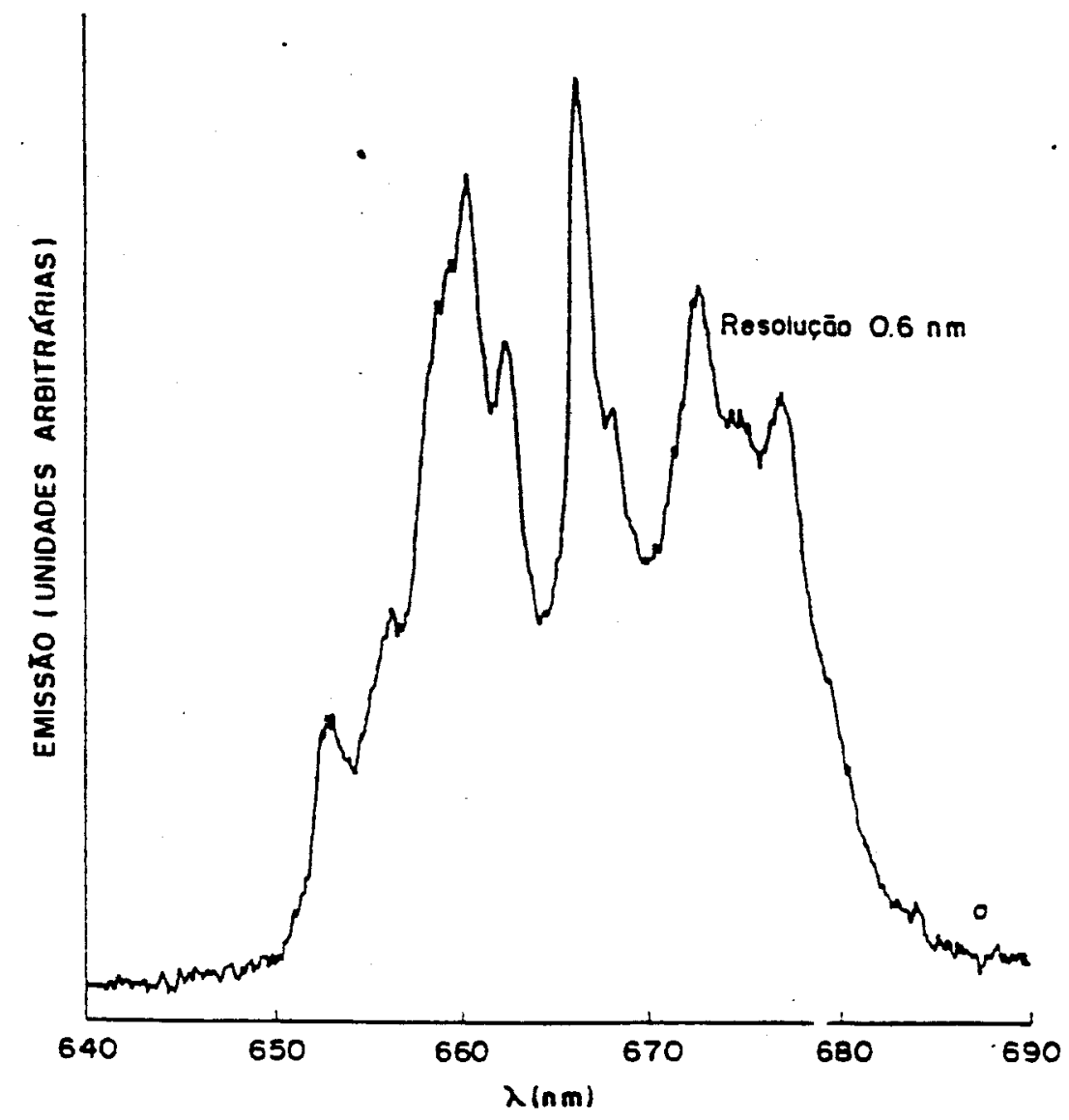

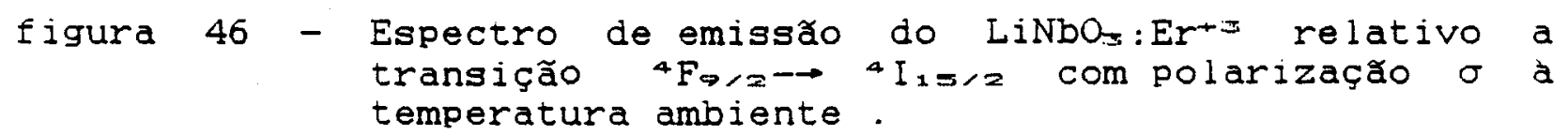

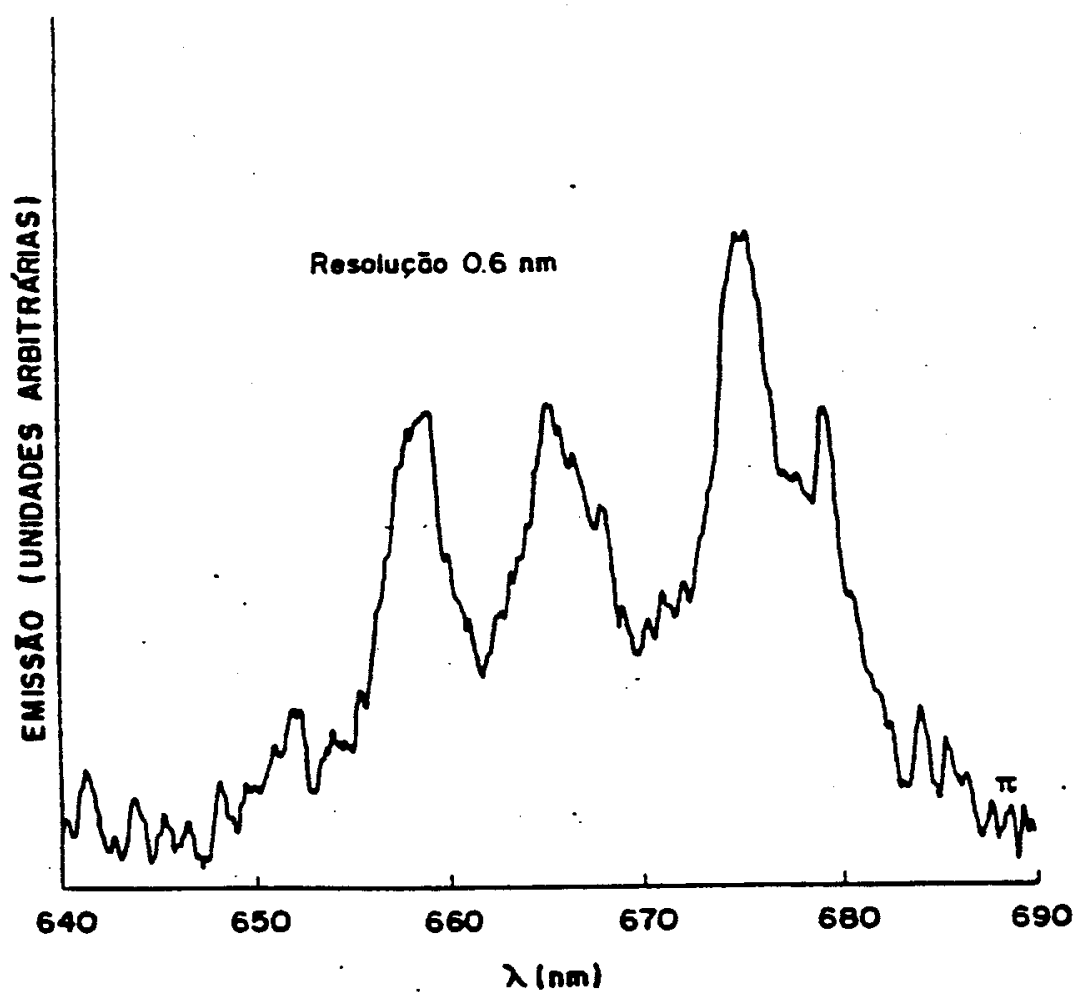

figura 47 - Espectro de emissão do LiNbOs:Er-3 relativo a transição ${ }^{4} \mathrm{~F}_{\theta / 2} \rightarrow{ }^{4} \mathrm{I}_{13 / 2}$ com polarizaçåo $\pi$ à temperatura ambiente. 


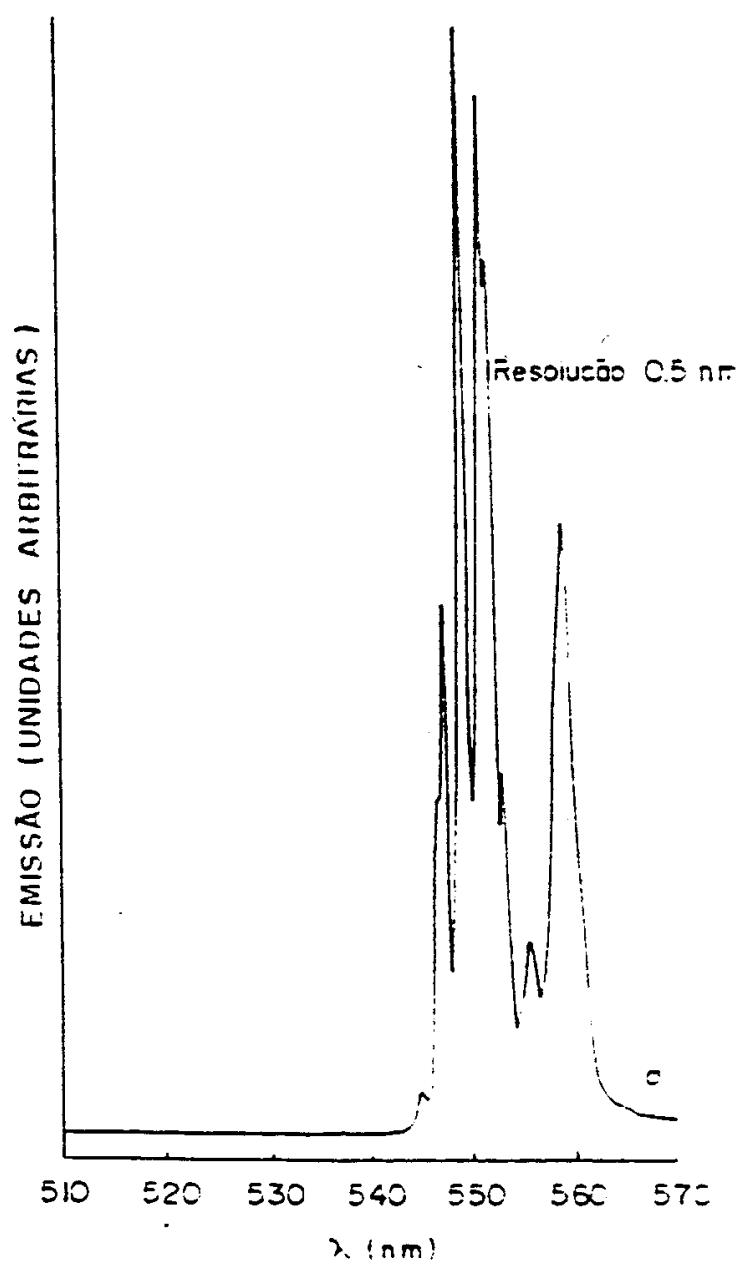

figura 48 - Espectro de emissåo do LiNbos:Er+3 relativo as transições ${ }^{2} \mathrm{H}_{11, z} \rightarrow{ }^{4} \mathrm{I}_{15 / 2}$ e ${ }^{4} \mathrm{~S}_{3 / 2} \rightarrow{ }^{4} \mathrm{I}_{13 / 2} \mathrm{com}$ polarizaçåo $\sigma$ a temperatura de nitrogênio liquido.

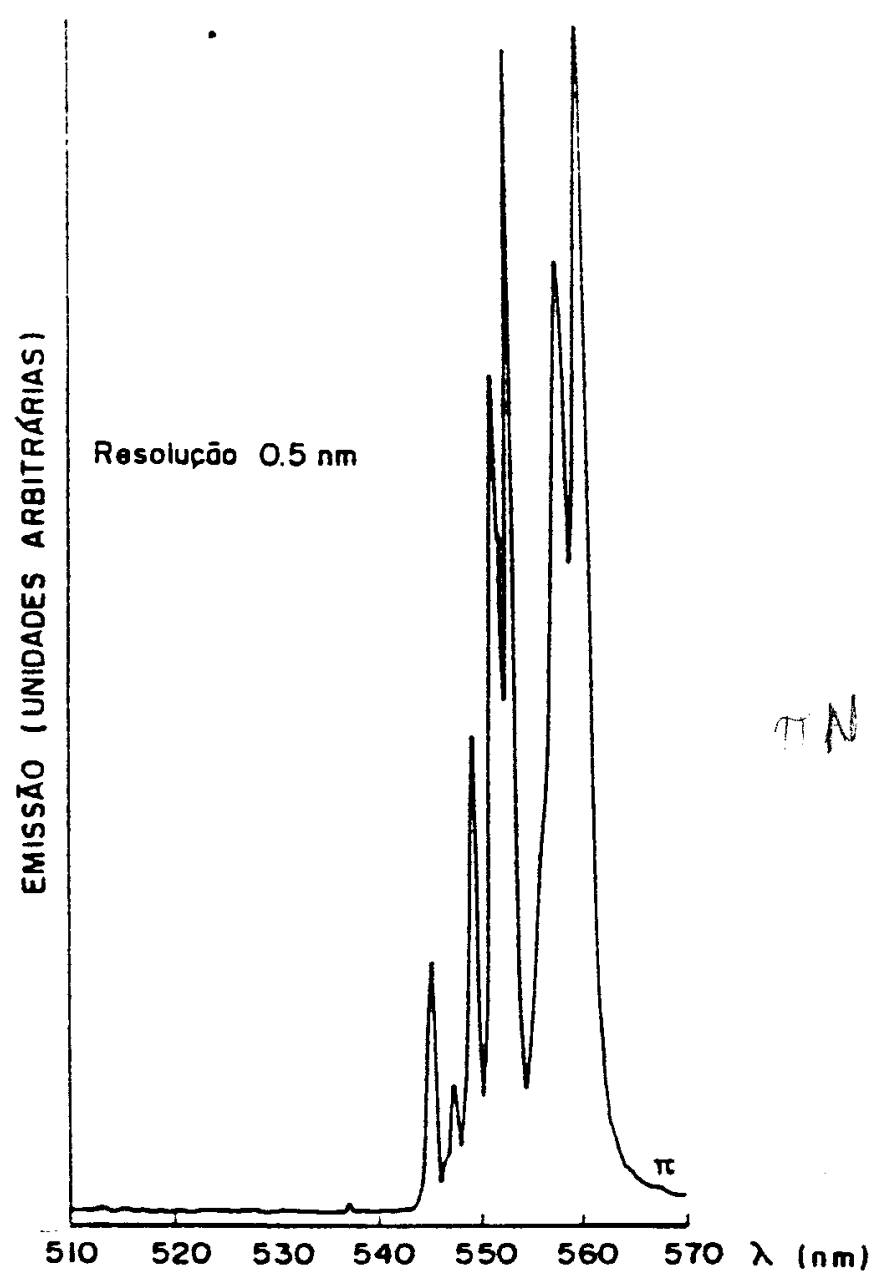

figura 49 - Espectro de emissao do LiNbOs:Er $\rightarrow 3$ relativo as transiçסes $\mathrm{z}_{11 / 2} \rightarrow{ }^{4} \mathrm{I}_{13 / 2}$ e ${ }^{4} \mathrm{~S}_{3 / 2} \rightarrow{ }^{4} \mathrm{I}_{15 / 2} \mathrm{com}$ polarizaçao $\pi$ a temperatura de nitrogenio liquido. 


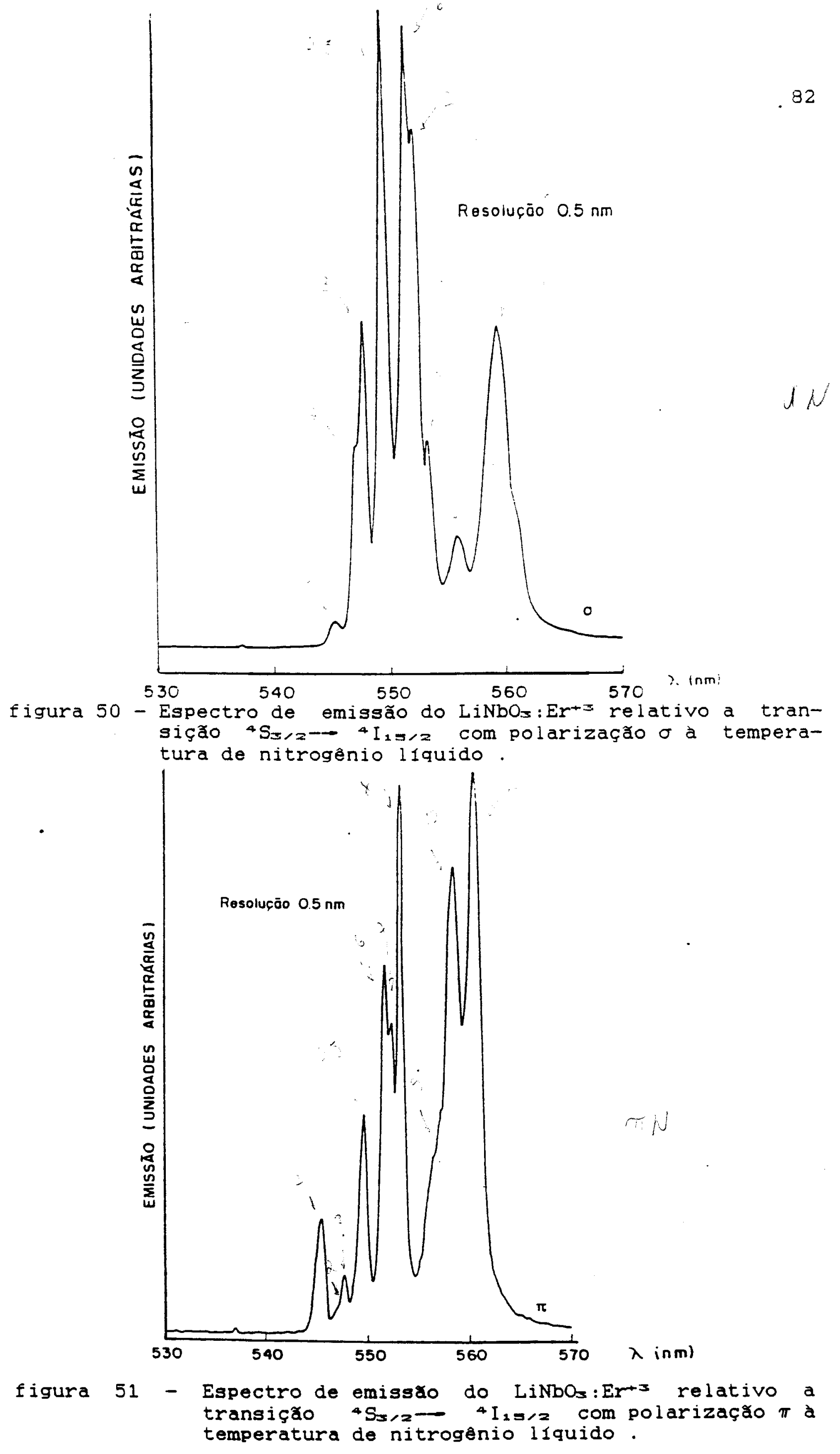




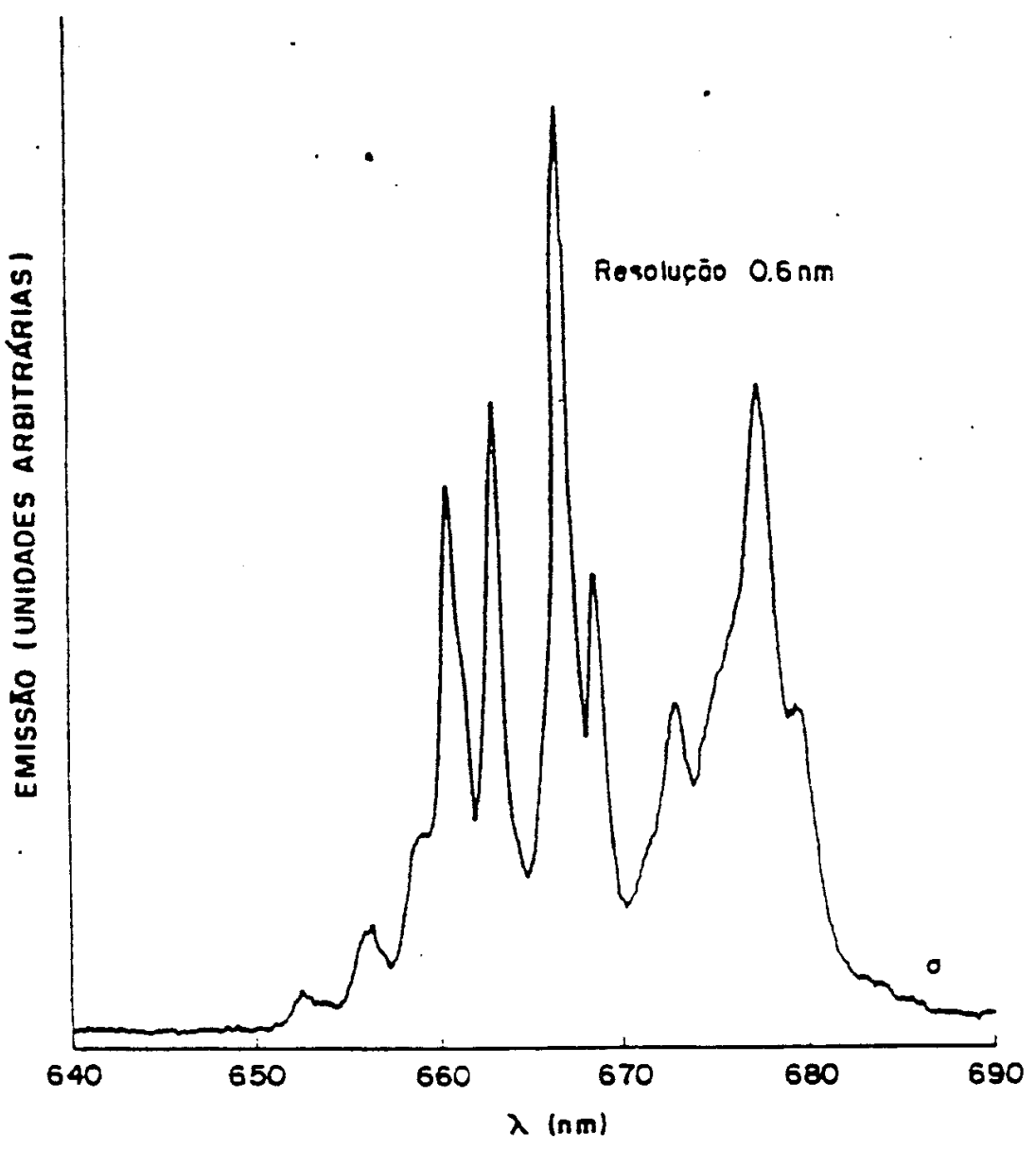

figura 52 - Espectro de emissăo do LiNbos:Er+x relativo a transição ${ }^{4} \mathrm{~F}_{9 / 2}-{ }^{4} \mathrm{I}_{1=/ 2}$ com polarizaçăo $\sigma$ à temperatura de nitrogênio liquido.

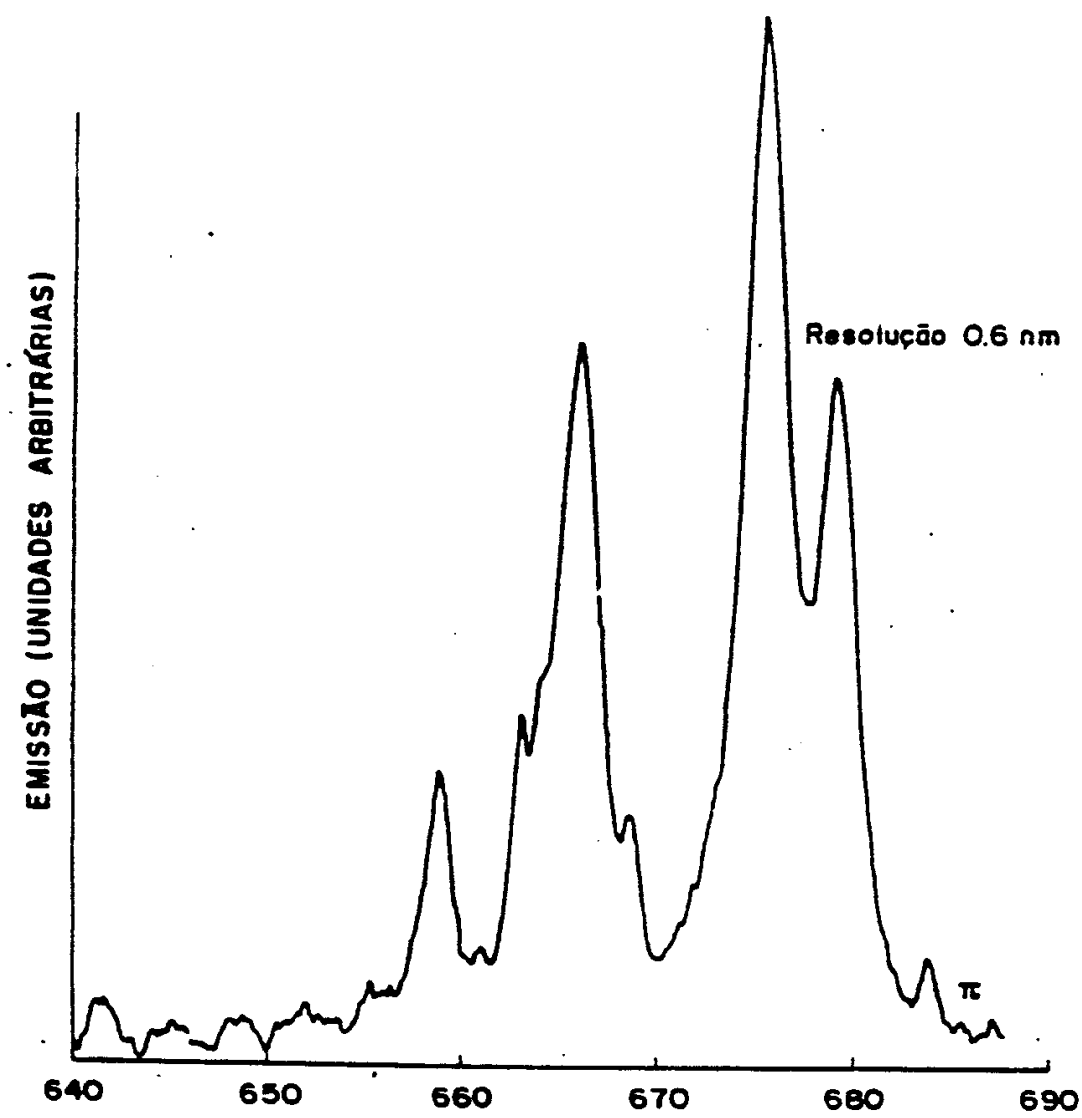

figura 53 - Espectro de emissa do LiNbos:Er 3 relativo a transiçåo ${ }^{4} \mathrm{~F}_{9 / 2} \rightarrow \mathrm{I}_{1=/ 2}$ com polarizaçå $\pi$ à temperatura de nitrogenio liquido. 
TABELA 18 - Energias relativas a transiçăo $x H_{1,2}-{ }^{4} I_{1 s, z}$

\begin{tabular}{|c|c|c|}
\hline POLARIZAÇAO & $\lambda(\mathrm{nm})$ & ENERGIA $\left(\mathrm{cm}^{-1}\right)$ \\
\hline \multirow{15}{*}{$\sigma$} & 521.7 & 19168.1 \\
\hline & 522.0 & 19157.1 \\
\hline & 522.9 & 19124.1 \\
\hline & 525,3 & 19036.7 \\
\hline & 526,6 & 18989.7 \\
\hline & 528.4 & 18925.1 \\
\hline & 529.2 & 18896.4 \\
\hline & 530.7 & 18843.0 \\
\hline & 531.8 & 18804.1 \\
\hline & 532.8 & 18768,8 \\
\hline & 535.8 & 18663,7 \\
\hline & 535,9 & 18660.2 \\
\hline & 536.5 & 18639.3 \\
\hline & 536,8 & 18628.9 \\
\hline & 537,0 & 18622.0 \\
\hline \multirow{9}{*}{$\pi$} & 521.5 & 19175.5 \\
\hline & 525.6 & 19025,9 \\
\hline & 526.4 & 18997.0 \\
\hline & 527,7 & 18950.2 \\
\hline & 529.8 & 18875.0 \\
\hline & 531.4 & 18818.2 \\
\hline & 536.0 & 18656.7 \\
\hline & 537.0 & 18622.0 \\
\hline & 538,0 & 18587.4 \\
\hline
\end{tabular}


TABELA 19 - Energias relativas a transiçăo ${ }^{4} \mathrm{~S}_{3 / 2}-{ }^{4} I_{1=12}$

\begin{tabular}{|c|c|c|c|}
\hline POLARIZAGAO & $\lambda(\mathrm{nm})$ & & ENERGIA $\left(\mathrm{cm}^{-1}\right)$ \\
\hline \multirow{10}{*}{$\sigma$} & 545.2 & ' & 18341.9 \\
\hline & 547.1 & & 18278.2 \\
\hline & 547.7 & & 18258.2 \\
\hline & 549.6 & & 18195.1 \\
\hline & 551,6 & & 18129.1 \\
\hline & 552.3 & & 18106.1 \\
\hline & 553.3 & & 18073,4 \\
\hline & 555.9 & & 17988,8 \\
\hline & 559.5 & & 17873.1 \\
\hline & 560.4 & . & 17844.4 \\
\hline \multirow{11}{*}{$\pi$} & 545.4 & ' & 18335.2 \\
\hline & 5.47 .0 & $\therefore$ & 18281.5 \\
\hline & 547.7 & & 18258.2 \\
\hline & 549.6 & & 18195.1 \\
\hline & 551.7 & & 18125.8 \\
\hline & 552,4 & & 18102.8 \\
\hline & 553.3 & & 18073,4 \\
\hline & 556,5 & & 17969.5 \\
\hline & 557,3 & & 17943.7 \\
\hline & 558.5 & & 17905.1 \\
\hline & 560.6 & & 17838.0 \\
\hline
\end{tabular}


TABELA 20 - Energias relativas a transiçăo ${ }^{4} F_{9 / z} \rightarrow{ }^{4} I_{1=/ z}$

\begin{tabular}{|c|c|c|}
\hline POLARIZAÇAO & $\lambda(\mathrm{nm})$ & ENERGIA $\left(\mathrm{cm}^{-1}\right)$ \\
\hline \multirow{18}{*}{$\sigma$} & 652.4 & 15328.0 \\
\hline & 656.2 & 15239,3 \\
\hline & 659.1 & 15172.2 \\
\hline & 660.5 & 15140,0 \\
\hline & 661.2 & 15124.0 \\
\hline & 663.0 & 15083.0 \\
\hline & 664.0 & 15060.2 \\
\hline & 666.0 & 15015,0 \\
\hline & 666.8 & 14997.0 \\
\hline & 668.6 & 14956.6 \\
\hline & 672.9 & 14861.0 \\
\hline & 675.2 & 14810.4 \\
\hline & 675.9 & 14795.1 \\
\hline & 676.4 & 14784,2 \\
\hline & 677.5 & 14760.1 \\
\hline & 679.1 & 14725.4 \\
\hline & 679.4 & 14718.9 \\
\hline & 679.7 & 14712,4 \\
\hline \multirow{8}{*}{$\pi$} & 658.9 & 15176.8 \\
\hline & 661.0 & 15128.6 \\
\hline & 663,1 & 15080.7 \\
\hline & 664.2 & 15055.7 \\
\hline & 664.5 & 15048.9 \\
\hline & 666.2 & 15010.5 \\
\hline & 668.6 & 14956.6 \\
\hline & 671.2 & 14898.7 \\
\hline
\end{tabular}


continuação

\begin{tabular}{llc}
\hline POLARIZAGAO & $\lambda(\mathrm{nm})$ & ENERGIA $\left(\mathrm{cm}^{-1}\right)$ \\
\hline & 671,9 & 14883,2 \\
& 673.1 & 14856,6 \\
$\pi$ & 675.5 & 14803,8 \\
& 679.2 & 14723,2 \\
683,9 & 14622,0
\end{tabular}

\subsection{TEMPO DE VIDA DE LUMINESCENNCIA}

As medidas de tempo de vida foram feitas usando polarizaçăo $\propto$ para conseguirmos todas as emissões sem previlegiarmos nenhuma. Na tabela temos os resultados medidos a temperatura ambiente e de nitrogenio liquido (77K). 
TABELA 21 - Medida do tempo de vida dos estados luminescentes a temperatura ambiente $e 77 \mathrm{~K}$.

\begin{tabular}{|c|c|c|c|}
\hline TRANS I ÇAO & $\lambda(n m)$ & $\tau_{\text {TEMP. AME. }}$ ( $\mu S$ ) & $\tau>>$ ( $\mu S)$ \\
\hline \multirow{11}{*}{${ }^{4} S_{X}=2-{ }^{4} I_{1}=2=$} & 545.3 & 60.0 & 120.0 \\
\hline & 547.0 & 60.0 & 120,0 \\
\hline & 547.7 & 60.0 & 120.0 \\
\hline & 549,6 & 60.0 & 120.0 \\
\hline & 551.7 & 60,0 & 120.0 \\
\hline & 552.3 & 60.0 & 120.0 \\
\hline & 553.3 & 60.0 & 120.0 \\
\hline & 556.0 & 60.0 & 120.0 \\
\hline & 557,3 & 60.0 & 120.0 \\
\hline & 558,5 & 60,0 & 120.0 \\
\hline & 560.6 & 60.0 & 120,0 \\
\hline \multirow{16}{*}{${ }^{4} F_{9 / 2}-C^{4} I_{1}=12$} & 652.4 & - & 40.0 \\
\hline & 56.2 & - & 40,0 \\
\hline & 558.9 & - & 60.0 \\
\hline & 663.1 & - & 60.0 \\
\hline & 664.5 & - & 40.0 \\
\hline & 666.0 & - & 40.0 \\
\hline & 666.2 & - & 40,0 \\
\hline & 666.8 & - & 60,0 \\
\hline & 668.6 & - & 60.0 \\
\hline & 671.2 & - & 60.0 \\
\hline & 671.9 & - & 40.0 \\
\hline & 673.1 & - & 40.0 \\
\hline & 675.5 & - & 60.0 \\
\hline & 677,5 & - & 40.0 \\
\hline & 679.2 & - & 40.0 \\
\hline & 683,9 & - & 20.0 \\
\hline
\end{tabular}




\section{ANÁLISE E DISCUSSTO DOS RESULTADOS EXPERIMENTAIS}

\subsection{INTRODUCAO}

Este capitulo esta dividido em quatro partes : 5.2 Avaliaçao dos efeitos dos tratamentos térmicos. 5.3 Analise dos espectros de absorção, 5.4 Análise dos espectros de luminescência e 5.5 Análise do tempo de vida dos estados luminescentes.

\subsection{AVALIAÇAO DOS EFEITOS DOS TRATAMENTOS TERMICOS}

A melhor eficiencia para a diminuiçăo da concentraçăo do $\mathrm{Fe}^{+2}$ e de $\mathrm{OH}^{-}$foi obtida atraves do tratamento térmico numa atmosfera de oxigénio. Pelas figuras 20 e 21 vemos que depois deste tratamento térmico tanto as concentraçós de $\mathrm{Fe}^{+\mathrm{z}}$ e $\mathrm{OH}^{-}$ se reduzem a niveis praticamente nao detectaveis pelas técnicas de absorçăo otica. Portanto numa primeira analise a eficiência do tratamento térmico numa atmosfera de oxigennio é superior a $95 \%$.

Um fato interessante que deve ser ressaltado destes tratamentos térmicos é o comportamento da concentraçăo do $\mathrm{Fe}^{+\pi}$. A principio pensavamos que o tratamento térmico gerava uma oxidação do $\mathrm{Fe}^{+z}$. Esta idéia, entretanto, começou a ser questionada quando, ao fazermos medidas de RPE, observanos que a concentraçă de $\mathrm{Fe}^{+3}$ nå se alterava com os tratamentos térmicos.

o mecanismo exato que descreve este comportamento ainda nao e conhecido. Na literatura existem muitas hipoteses que 
tentam explicar ofato, mas nehuma tem comprovaçăo experimental. Através de discussoes com os professores Otaciro Rangel e Maria Cristina Terrile foram criadas as seguintes hipoteses :

-1- quando fazemos o tratamento termico transiormamos $\circ \mathrm{Fe}^{-2}$ em ferro com valência diferente de +3 , como por exemp $10+4$ que não é detectado por RPE ;

-2- o elétron cedido pelo $\mathrm{Fe}^{+2}$ vai para $\mathrm{Nb}^{+=}$transiormando-o em $\mathrm{Nb}^{+4}$ e formam-se pares $\mathrm{Fe}^{+-3}-\mathrm{Nb}^{+4}$ que nå såo detectaveis por RPE . :

Esta hipotese baseada na formação de $\mathrm{Nb}^{+4}$ vem de alguns resultados obtidos pela prof*. Terrile. Ela observou que bombeando uma pequena area de um cristal de LiNbOs com laser de argônio, à baixas temperaturas, e medindo simultaneamente 0 espectro de RPE detecta-se geraçao de lons $\mathrm{Nb}^{+4}$. Este fato a prof". Terrile atribuiu a transferencia de carga do $\mathrm{Fe}^{+2}$ para 0 $\mathrm{Nb}^{+}=$: visto que estes dois lons possuem uma interaçăo muito forte entre eles, chegando até a partilhar um eletron. Segundo $=$ Y.V. Vladimirtsev e coautores, esta interaçăo e que origina a banda em $485 \mathrm{~nm}$ que é proporcional a concentração de $\mathrm{Fe}^{+2}$.

o tratamento térmico no ar em cadinho de ceramica e bem menos eficiente do que os realizados no ar e em atmosfera de oxigenio. E necessario muitas horas para se conseguir algum resultado relevante.

No cadinho de niquel os resultados sao rapidos e com uma eficiencia aproximadamente igual a obtida com tratamento termico no cadinho de ceramica em atmosfera de oxigênio. Entretanto o cristal muda de coloração indo do rosa para um tom de amarelo.

o oxido de niquel é um conhecido catalisador de reaçres quimicas. A presença de niquel em cristais de LiNbos pode ser 
detectada através de uma coloraçăo amarelada que o cristal apresenta. Portanto entendemos que os consecutivos tratamentos térmicos realizados em altas temperaturas na atmosiera ambiente acabam por oxidar a superficie do cadinho e este oxido de niquel em contato com o cristal nåo so catalisa o processo de oxidaçăo do $\mathrm{Fe}^{+2}$ como tambem, por participar da reaçå, acaba por impurificar o cristal com niquel.

Desta forma o metodo mais adequado e mais eficiente e o tratamento com cadinho de ceramica em atmosiera de oxigenio, pois leva as concentraçoes de $\mathrm{OH}^{-}$e $\mathrm{Fe}^{+2}$ a nlveis bem baixos em pouco tempo. entretanto é bom ressaltar que as amostras puras e dopadas com terras-rara crescidas pelo grupo de crescimento de cristais do IFQSC, ja naturalmente apresentam baixa concentração de $\mathrm{Fe}^{+\mathrm{z}}$.

\subsection{ANÁLISE DO ESPECTRO DE ABSORÇAO}

Como vimos na seção 2.5 , impurezas catiônicas quando entram no $\mathrm{LiNbO}$ podem ocupar très sitios : $\mathrm{Li}^{+1}$, $\mathrm{Nb}^{+\infty}$ ou vacancias estruturais da rêde. Estas trés possibilidades têm a mesma simetria, $C_{3}$. Os campos, é claro, såo levemente diferentes pois temos a substituiçăo de lons com valencias diferentes, no caso de terras-rara trivalentes temos +3- +1, +3- +5 e $+3 \rightarrow 0$. mas este fato pode resultar apenas numa pequena bifurcaçao nas linhas.

Desta forma, quando o Er+ entra nesta rede fica sujeito a uma simetria $C:$ e a familia de simetrias que origina $C=$ neste cristal esta descrita na figura 54 .

Conhecendo a simetria local $C_{3}$ e a familia de simetrias que leva a $C_{3}$ podemos fazer uma primeira avaliaçăo do espectro deste 
sistema.

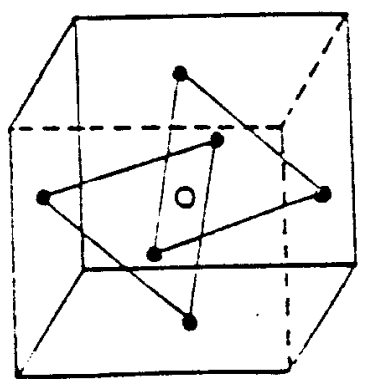

On

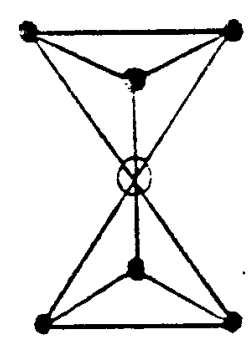

Dsa

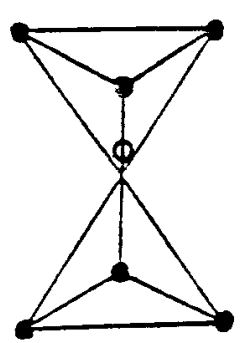

Cav

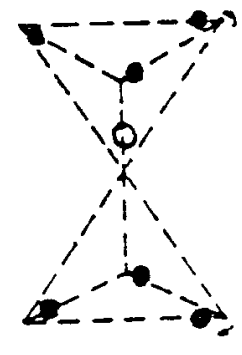

C.3

figura 54 - Familia de simetrias que geram $C=$ neste cristal.

Conhecendo a simetria local $C=$ e a familia de simetrias que leva a $C_{s}$ podemos fazer uma primeira avaliaçăo do espectro deste sistema.

Na figura 55 temos o desdobramento dos niveis na familia de simetrias de $\mathrm{C}=$.

Como para todas as transições de absorção o espectro $\sigma$ e igual ao $\alpha$, as transições sao dipolares elétricas. Na tabela 22 temos um resumo geral dos operadores dipolares eletricos nestas simetrias e as regras de seieção para as transiçoes, considerando apenas o estado de mais baixa energia do fundamental $I_{1 s / 2}$. Para sabermos quem e a representação irredutıvel que descreve o estado de mais baixa energia recorremos a teoria do campo cristalino. Atraves desta teoria conseguimos mapear o comportamento das representaçðes irredutiveis em relaçăo ao espaçamento entre elas para todas as possibiliadades de deformaça de um campo cubico. O resultado deste estudo para o estado fundamental do Er+ esta na figura 56 . Com este estudo e os resultados experimentais o estado de menor energia numa simetria On é $\Gamma_{>} \circ$ que significa numa simetria $C_{3} \operatorname{ser}\left(\Gamma_{4}+\Gamma_{3}\right)$ ou $\Gamma_{4}=$. Os detalhes deste calculo usando teoria do campo cristalino estão na seçăo $5 \cdot 3 \cdot 3$. 


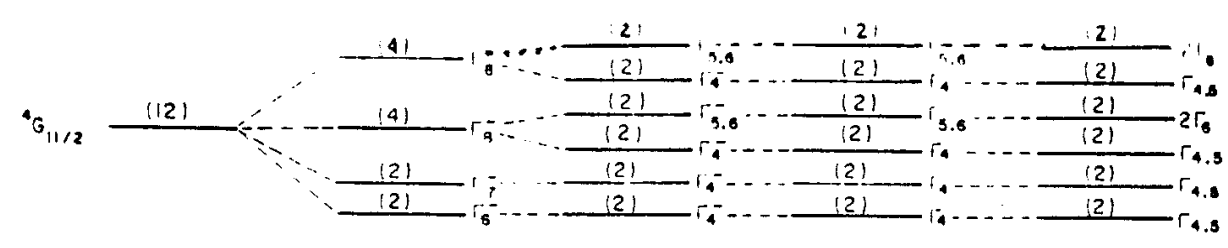

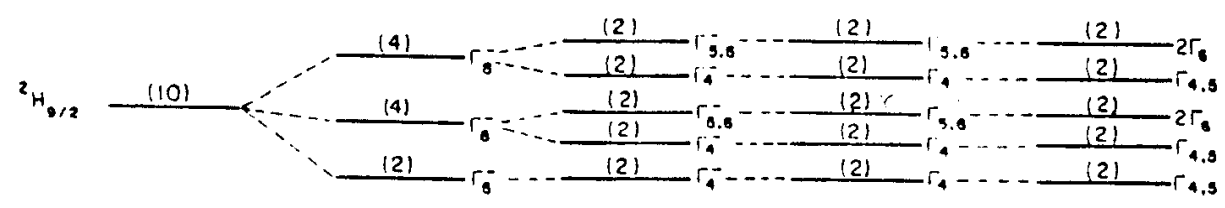

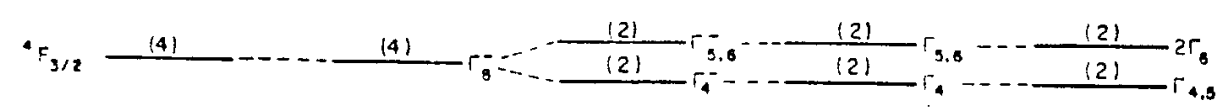

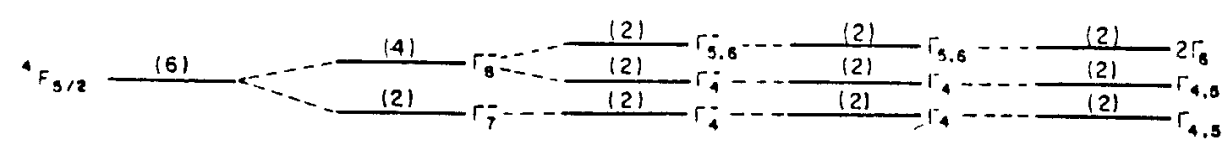

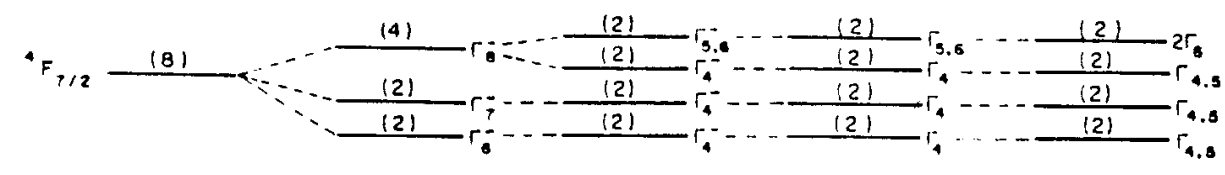
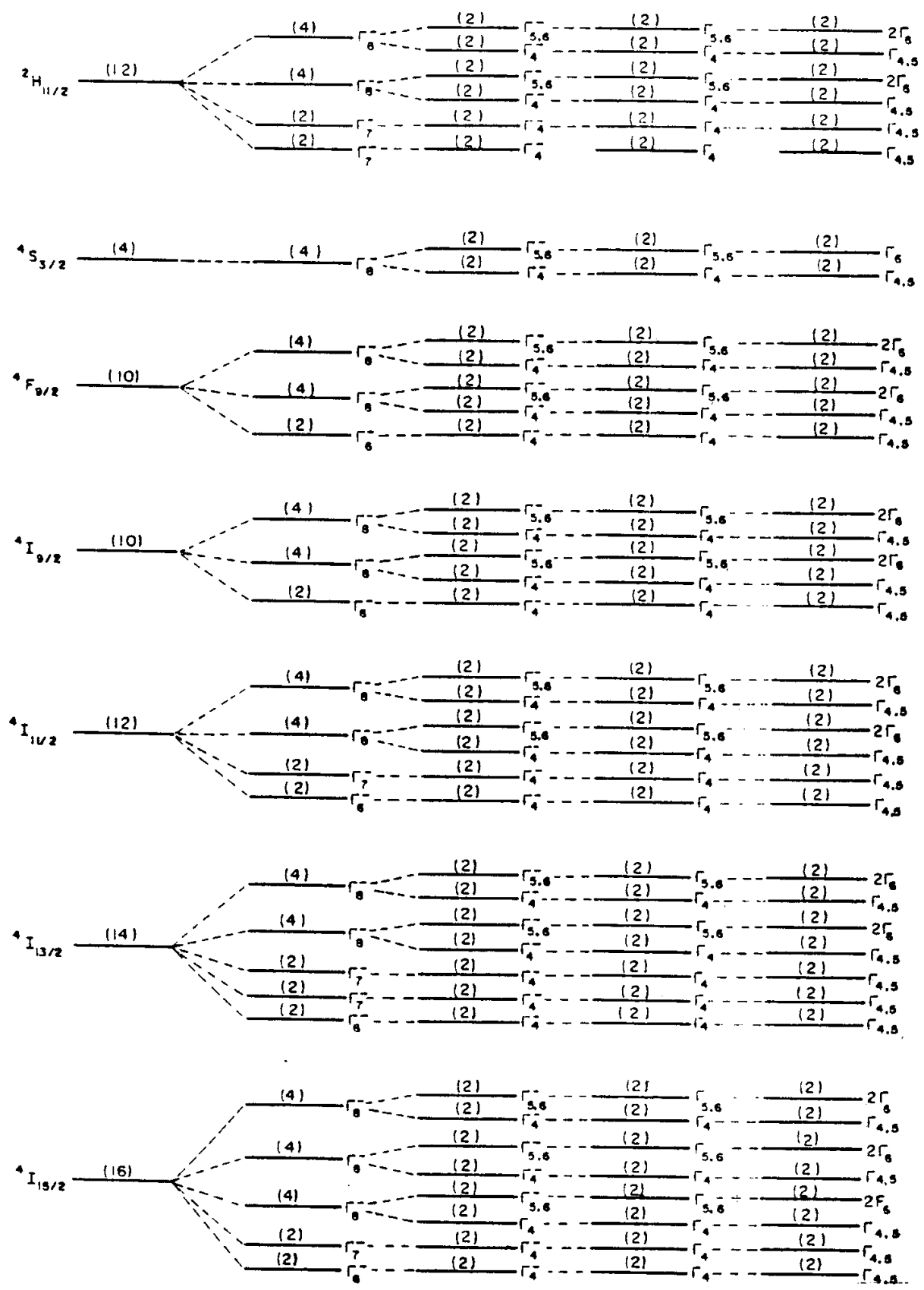
TABELA 22 - Caracterizaçăo das transiçðes do $\mathrm{Er}^{+*}$ nas simetrias On, $\mathrm{D}=a, \mathrm{C}_{3} \sim$ e $\mathrm{C}_{3}$

\begin{tabular}{llll}
\hline SIM. O.D.E.(dir.) & REPR. DO EST. & TRANS.PERM. POL \\
& FUND. DO Er &
\end{tabular}

on $\quad \Gamma_{4}^{-}(x, y, z) \quad \Gamma_{0}^{-}$ou $\Gamma_{7}^{-}$

\begin{tabular}{lllll}
\hline Dsa & $\Gamma_{z-}^{-}(z)$ & $\Gamma_{4}^{-}$ & - & - \\
& $\Gamma_{x-}-(x, y)$ & & -
\end{tabular}

\begin{tabular}{|c|c|c|c|c|}
\hline $\mathrm{C}=\square$ & $\begin{array}{l}\Gamma_{1}(z) \\
\Gamma=(x, y)\end{array}$ & $\Gamma_{4}$ & $\begin{array}{c}\Gamma_{4}-\Gamma_{4} \\
\Gamma_{4}-\Gamma_{4}+\left(\Gamma_{5}+\Gamma_{6}\right)\end{array}$ & $\begin{array}{l}\pi \\
\propto \sigma\end{array}$ \\
\hline
\end{tabular}

$\Gamma_{1}(z)$

$C x$ $(\Gamma=+\Gamma \rightrightarrows)(X, Y)$
$\left(\Gamma_{4}+\Gamma_{3}\right)-\left(\Gamma_{4}+\Gamma_{3}\right) \quad \pi$

$\left(\Gamma_{4}+\Gamma=\right)$

$\left(\Gamma_{4}+\Gamma_{3}\right)-\left(\Gamma_{4}+\Gamma_{3}\right)+\Gamma_{6} \propto \sigma$

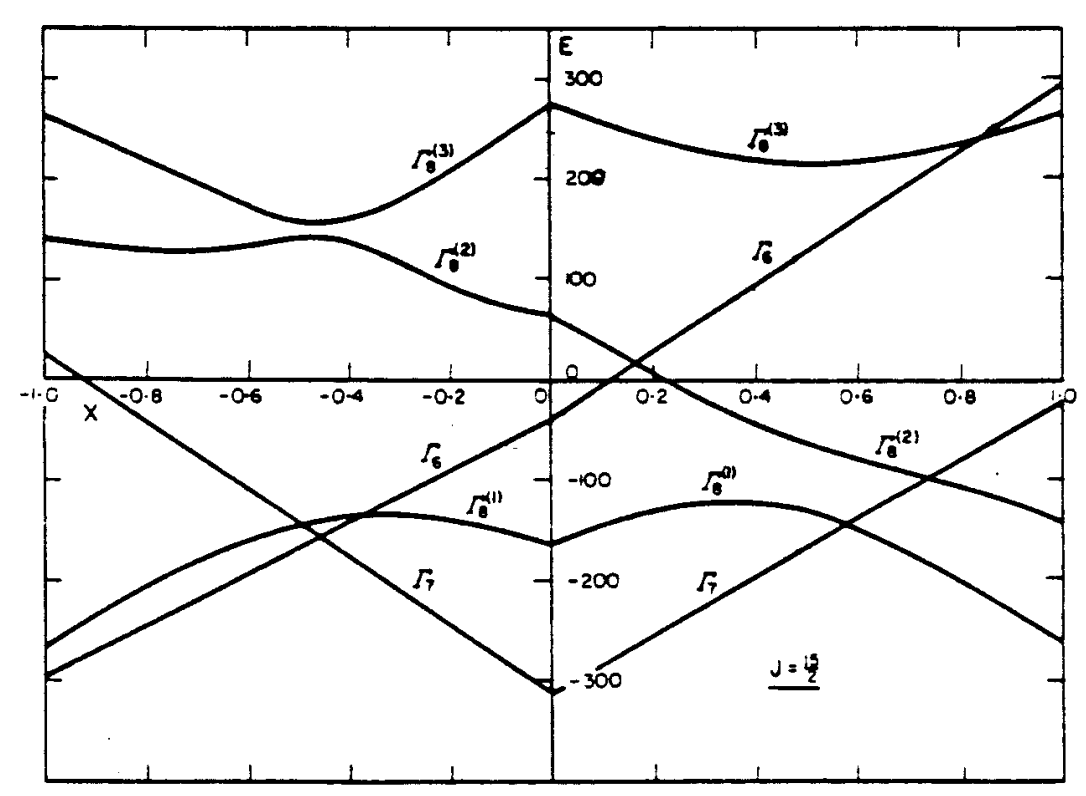

figura 56 - Grafico do comportamento das representaçбes irredutiveis de um estado com $\mathrm{J}=15 / 2$ para diversos tipos de deformaçăo cúbica. 
Como se pode ver na tabela 22 , as transiçoes passam a ser permitidas depois da simetria Csu. quando a vizinhança do ion perde a simetria de inversão devido do deslocamento do mesmo para fora do centro. Com apenas as informaçðes das simetrias $C_{3} e$ Car nada pode-se concluir sobre as intensidades pois, as regras de seleçåo para ambas as simetrias levam as mesmas transições.

$\mathrm{Na}$ tabela 23 temos os resultados do numero de linhas que devem ser observadas com suas respectivas polarizaçరes para cada transiçăo de absorçăo. E bom ressaltar que as lininas que aparecem em $\pi$ aparecem tambem em $\sigma \alpha$, mas as de $\sigma \alpha$ não aparecem em $\pi$.

TABELA 23 - Número de transições e suas respectivas polarizaçðes para os niveis do érbio trivalente numa simetria $C=$.

\begin{tabular}{|c|c|c|}
\hline TRANS . & NUMERO DE IINHAS & POL. \\
\hline${ }^{4} I_{1}=/ 2 \rightarrow 4 I_{1}=r 2$ & $\begin{array}{l}5 \\
7\end{array}$ & $\begin{array}{l}\pi \\
\sigma \alpha\end{array}$ \\
\hline${ }^{4} I_{15 / 2} \rightarrow{ }^{4} I_{11 / 2}$ & $\begin{array}{l}4 \\
6\end{array}$ & $\begin{array}{l}\pi \\
\sigma \alpha\end{array}$ \\
\hline${ }^{4} I_{15 / 2}=-{ }^{4} I_{0 / 2}$ & $\begin{array}{l}3 \\
5\end{array}$ & $\begin{array}{l}\pi \\
\sigma \propto\end{array}$ \\
\hline${ }^{4} I_{1}=12 \rightarrow{ }^{4} F_{912}$ & $\begin{array}{l}3 \\
5\end{array}$ & $\begin{array}{l}\pi \\
\sigma \propto\end{array}$ \\
\hline${ }^{4} I_{1}=12-{ }^{4} S_{312}$ & $\begin{array}{l}1 \\
2\end{array}$ & $\begin{array}{l}\pi \\
\sigma \alpha\end{array}$ \\
\hline
\end{tabular}




\section{continuação}

\begin{tabular}{|c|c|c|}
\hline TRANS . & NUMERO DE LINHAS & POL. \\
\hline${ }^{4} \mathrm{I}_{1}=12-\rightarrow \mathrm{H}_{11,2}$ & $\begin{array}{l}4 \\
6\end{array}$ & $\begin{array}{l}\pi \\
\sigma \alpha\end{array}$ \\
\hline${ }^{4} I_{1} \leq r x \rightarrow{ }^{4} F_{>12}$ & $\begin{array}{l}3 \\
4\end{array}$ & $\begin{array}{l}\pi \\
\sigma \propto\end{array}$ \\
\hline${ }^{4} I_{1=/ 2}-{ }^{4} F_{5 / 2}$ & $\begin{array}{l}2 \\
3\end{array}$ & $\begin{array}{l}\pi \\
\sigma \propto\end{array}$ \\
\hline${ }^{4} I_{1}=1=--{ }^{4} \mathrm{~F}=12$ & $\begin{array}{l}1 \\
2\end{array}$ & $\begin{array}{l}\pi \\
\sigma \alpha\end{array}$ \\
\hline${ }^{4} I_{1}=12->2 H_{0}=0$ & $\begin{array}{l}3 \\
5\end{array}$ & $\begin{array}{l}\pi \\
\sigma \propto\end{array}$ \\
\hline${ }^{4} I_{1}=/ 2 \rightarrow{ }^{4} G_{11 / 2}$ & $\begin{array}{l}4 \\
6\end{array}$ & $\begin{array}{l}\pi \\
\sigma \alpha\end{array}$ \\
\hline
\end{tabular}

\subsubsection{CALCULO DAS ENERGIAS DOS ESTADOS DO Er $\mathbf{r}^{+3}:$ LiNbO=}

A identificaçå das transiçoes relativas ao estado de mais baixa energia do fundamental para os niveis excitados pode ser feita atraves dos estudos da teoria de grupos, pois com ele conhecemos o número de linhas esperado e a polarizaçao das transiçסes. No entanto esta análise pode ficar um tanto quanto complicada quando se tem um fundamental com degenerescencia muito alta , como é o caso do érbio trivalente $(J=15 / 2)$. Neste caso o melhor a ser feito é procurar saber se as energias que julgamos ser relativas aos niveis do erbio nesta matriz sao 
coerentes com algum conjunto de parametros que descrevem a Hamiltoneana deste sistema.

Um tratamento analitico do comportamento de eletrons $f$ em campos de simetria cubica foi feito por Lea e coautores ${ }^{* 1}$. Neste trabalho Lea escreve a Hamiltoneana do campo em termos de operadores equivalentes e constantes que podem ser ajustadas de forma a se obter todas as deformações tetragonais possiveis do campo cristalino. Desta forma ele consegue mapear o comportamento relativo dos niveis de energia para terras-raras numa simetria cúbica.

De forma analoga montamos a Hamiltoneana de campo cubico com operadores equivalentes e adotando as mesmas constantes definidas por Lea , entretanto mudamos o sistema de eixos utilizados. Lea utiliza um sistema de eixos com eixo $z$ quatro vèzes degenerado o qual e util quando se deseja fazer distorções tetragonais no campo. Em nosso sistema vamos fazer distorções trigonais, pois o eixo $z$ é três vêzes degenerado, logo é adequado fazermos esta mudança.

Outro bom motivo para fazermos esta mudança de sistema de coordenadas é que utilizando o eixo tres vezes degenerado diminui o número de operadores equivalentes a ser acrescentado a Hamiltoneana cúbica para descrever o campo de $C_{3}$, pois as deformaçбes de reduça de simetria såo nesta direçăo, e desta forma diminui o número de constantes a serem ajustadas. Para um sistema de eixos com eixo $z$ très vêzes degenerado, a Hamiltoneana do campo cristalino pode ser escrita como :

$$
\begin{aligned}
\mathrm{H}_{\text {CRIBTAL }}=-(2 / 3) \mathrm{B}_{4}^{\circ}\left[\mathrm{O}_{4}^{\circ}-20(2)^{1 / 2} \mathrm{O}_{4}\right]+ \\
(16 / 9) \mathrm{B}_{6}^{\circ}\left[\mathrm{O}_{6}^{0}+\left(\left(35(2)^{1 / 2}\right) / 4\right) \mathrm{O}_{6}=+(77 / 8) \mathrm{O}_{6}^{6}\right]
\end{aligned}
$$


onde

$$
\begin{aligned}
& \mathrm{O}_{4}{ }^{\circ}=35 \mathrm{Jz}^{4}-30 \mathrm{~J}(\mathrm{~J}+1) \mathrm{J}_{\mathrm{z}}{ }^{2}+25 \mathrm{~J}_{\mathrm{z}}{ }^{2}-6 \mathrm{~J}(\mathrm{~J}+1)+3 \mathrm{~J} z(\mathrm{~J}+1)= \\
& O_{4}:=1 / 4\left[J_{z}\left(J_{+}=+J_{-} \Xi\right)+\left(J_{+} \Xi J_{-} \Xi\right) J_{z}\right] \\
& \mathrm{O}_{\mathrm{s}}^{0}=231 \mathrm{Jz} \mathrm{z}^{4}-315 \mathrm{~J}(\mathrm{~J}+1) \mathrm{Jz}_{\mathrm{z}^{4}}+735 \mathrm{Jz}^{4}+105 \mathrm{~J} \mathrm{z}(\mathrm{J}+1)=\mathrm{Jz} \mathrm{z}^{2}+ \\
& 294 \mathrm{Jz}^{2}=-5 \mathrm{~J} \approx(\mathrm{J}+1)^{3}+40 \mathrm{~J}=(\mathrm{J}+1)^{2}-60 \mathrm{~J}(\mathrm{~J}+1) \\
& Q_{s}==1 / 4\left[( 1 1 J _ { z } = - 3 J _ { z } J ( J + 1 ) - 5 9 J _ { z } ) \left(J_{+} I_{+} J_{-}=+\right.\right. \text {. } \\
& \left.\left(J_{+}=+J_{-}=\right)\left(11 J_{z}=-3 J_{z} J(J+1)-59 J_{z}\right)\right] \\
& O_{6}^{6}=1 / 2\left(J_{+}{ }^{6}+J_{-} 6\right)
\end{aligned}
$$

e os coeficientes $\mathrm{B}_{4}^{\circ}$ e $\mathrm{B}_{6}^{\circ}$ sao as constantes que determinam - grau de perturbaçao da Hamiltoneana com eixos quatro vèzes degenerado dados por

$$
\begin{aligned}
& B_{4}^{\circ}=\frac{7}{16} \frac{Z e^{2}}{d^{5}} B_{4}\left\langle r^{4}\right\rangle \\
& B_{\infty}^{\circ}=\frac{3}{64} \frac{Z e^{2}}{d^{7}} \gamma_{4}\left\langle r^{\infty}\right\rangle
\end{aligned}
$$

onde d é distancia dos vizinhos mais proximos do ion terra-rara cuja carga é $z e, \quad\left\langle r^{4}\right\rangle$ é a parte radial da funçăo de onda do eletron $(d\rangle\langle r\rangle) e, \quad \beta, e$, săo constantes relacionadas com a parte angular da função de onda do eletron e vão depender do número quantico $\mathrm{J}$ do estado em estudo.

Por simplicidade vamos escrever a Hamiltoneana (60) da seguinte forma :

$$
\text { Herigtal }=-\frac{2}{3} \cdot B_{4}^{\circ} \mathrm{O}_{4}+\frac{16}{9} \mathrm{~B}_{6}^{\circ} \mathrm{O}_{6}
$$

onde

$$
\begin{aligned}
& O_{4}=0_{4}^{0}-20(2)^{1 / 2} O_{4}= \\
& O_{6}=0_{6}^{0}+\left(\left(35(2)^{1 / 2}\right) / 4\right) O_{6}^{3}+(77 / 8) O_{6}^{6}
\end{aligned}
$$


tabelados por Stevensira. Estas tabelas contêm fatores comuns a todos os elementos de matriz, $F(4)$ e $F(6)$, e estes são separados no presente cálculo a fim de manter os autovalores na mesma faixa numérica para todas as razões de têrmos de grau quatro e seis. Portanto podemos reescrever a Hamiltoneana do campo cristalino já expressando estes têrmos.

$$
\text { HERIMTAL }=-\frac{2}{3} B_{4}^{\circ} F(4) \frac{O_{4}}{F(4)}+\frac{16}{9} B_{6}^{\circ} F(6) \frac{O_{4}}{F(6)}
$$

Para cobrir todas as possibilidades de valores da razáo dos têrmos de grau quatro e seis definimos :

$$
\begin{aligned}
& B_{4} F(4)=W x \\
& B_{46} F(6)=W(1-|x|),
\end{aligned}
$$

onde $-1<x<1$.

De $(66)$ segue que

$$
\frac{B_{4}}{B_{6}}=\frac{x}{F(6)} \frac{F(4)}{(1-|x|)}
$$

desta forma quando $\left(B_{4} / B_{6}\right)=0$ temos $x=0$ e quando $\left(B_{4} / B_{6}\right)= \pm \infty$ temos $x= \pm 1$.

Reescrevendo a Hamiltoneana com as constantes definidas em (66) temos

$$
\mathrm{H}_{\text {ERIBTAL }}=\mathrm{W}\left[-\frac{2}{3} \times \frac{\mathrm{O}_{4}}{\mathrm{~F}(4)}+\frac{16}{9}(1-i \mathrm{xi}) \frac{\mathrm{O}_{4}}{\mathrm{~F}(6)}\right]
$$

Expressando a Hamiltoneana na forma (68) o têrmo dentro dos colchetes representa uma matriz quadrada de dimensao $2 \mathrm{~J}+1$, cujos autovetores correspondem a combinaçao mais geral de campos cristalinos com distorçres trigonais e os autovalores, quando multiplicados pelo fator de escala w revelam a abertura dos niveis de energia do fon quando colocado neste campo.

Com as tabelas do apendice $B$ pode-se construir as matrizes 
para cada estado definido pelo número quantico $J$ como funçăo de $x$ e atraves de um programa de computador para diagonalizar matrizes é possivel fazer graficos do comportamento do splitting dos niveis em função de $x$, como foi feito por Leain. Os resultados obtidos por Lea e reproduzidos por nos estão na figura 57 .
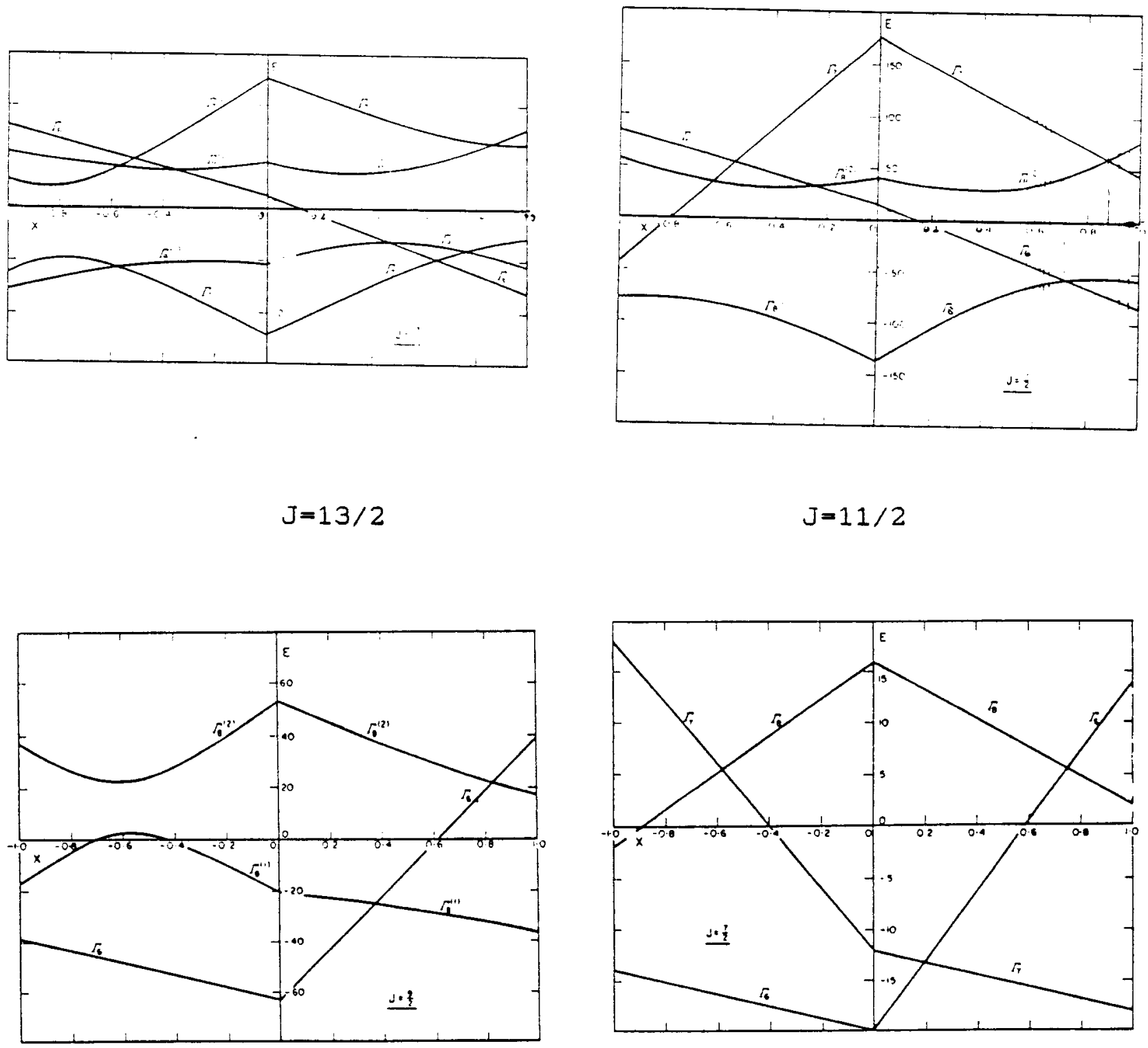
Com as previsóes feitas pela teoria de grupos e com os resultados experimentais podemos fazer uma previsão grosseira de quais seriam as energias que iriamos observar se colocassemos 0 mesmo ion sujeito a uma simetria cubica. Este resultado e os graficos da figura 57 nos levam a descobrir a um valor aproximado de $x$ que servira de base para o ajuste das constantes para a Hamiltoneana do campo com simetria $C_{3}$.

Para escrevermos a Hamiltoneana do campo para a simetria $C_{3}$ basta acrescentarmos a Hamiltoneana do campo cúbico o operador $\mathrm{O}^{\circ}$ :

$$
\text { HeFistal }=\mathrm{W}\left[-\frac{2}{3} \mathrm{~B}_{4}^{\circ} \mathrm{O}_{4}+\frac{16}{9} \mathrm{~B}_{6}^{\circ} \mathrm{O}_{6}+\mathrm{AO}_{2}^{\circ}\right]
$$

onde

$$
\mathrm{O}_{2}=3 \mathrm{~J}_{z}=-J(J+1)
$$

e A é uma constante a ser ajustada com os valores experimentais. Para deixar bem claro o procedimento utilizado para calcular os niveis de energia escolhemos dois exemplos ${ }^{4} F=/ z$ e ${ }^{4} F>r z$. A escolha destes dois estados, especificamente, se deve ao fato deles ilustrarem bem duas situaçðes diferentes que ocorrem durante este cálculo. O nivel ${ }^{4} F=/ z$ demonstra a situaçăo onde ficam determinadas as constantes WA $e$ WX $e$ nivel ${ }^{4} F_{7, z}$ demonstra a situação onde conseguimos determinar $W$, A e $x$.

No caso de $J=5 / 2$ temos a seguinte matriz: 


\begin{tabular}{|c|c|c|c|c|c|c|}
\hline & $\{5 / 2\rangle$ & $|3 / 2\rangle$ & $: 1 / 2\rangle$ & $i-1 / 2\rangle$ & $i-3 / 2>$ & $-5 / 2>$ \\
\hline$: 5 / 2\rangle$ & $-\frac{2}{3}+10 A$ & 0 & 0 & $-\frac{4}{3} 5 x$ & 0 & 0 \\
\hline$: 3 / 2>$ & 0 & $2 x-2 A$ & 0 & 0 & 0 & 0 \\
\hline$\{1 / 2\rangle$ & 0 & 0 & $-\frac{4}{3} x-8 A$ & 0 & 0 & $\frac{4}{3} 5 x$ \\
\hline$|-1 / 2\rangle$ & $-\frac{4}{3} 5 x$ & 0 & 0 & $-\frac{4}{3} x-8 A$ & 0 & 0 \\
\hline$:-3 / 2>$ & 0 & 0 & 0 & 0 & $2 x-2 A$ & 0 \\
\hline$:-5 / 2\rangle$ & 0 & 0 & $-\frac{4}{3} 5 x$ & 0 & 0 & $-\frac{2}{3} x+10 A$ \\
\hline
\end{tabular}

Note que podemos reorganizar esta matriz a fim de agrupar em blocos os estados que văo continuar interligados mesmo com a perturbação do campo.

\begin{tabular}{|c|c|c|c|c|c|c|}
\hline & $: 3 / 2>$ & $i-3 / 2\rangle$ & $: 5 / 2\rangle$ & $i-1 / 2\rangle$ & $|1 / 2\rangle$ & $i-5 / 2>$ \\
\hline$|3 / 2\rangle$ & $2 x-2 A$ & 0 & 0 & 0 & 0 & 0 \\
\hline$i-3 / 2>$ & 0 & $2 x-2 A$ & 0 & 0 & 0 & 0 \\
\hline $15 / 2\rangle$ & 0 & 0 & $-\frac{2}{3} x+10 A$ & $-\frac{4}{3} 5 x$ & 0 & 0 \\
\hline$i-1 / 2\rangle$ & 0 & 0 & $-\frac{4}{3} 5 x$ & $-\frac{4}{3} x-8 A$ & 0 & 0 \\
\hline$\{1 / 2\rangle$ & 0 & 0 & 0 & 0 & $-\frac{4}{3} x-8 A$ & $-\frac{4}{3} 5 x$ \\
\hline$i-5 / 2\rangle$ & 0 & 0 & 0 & 0 & 0 & $-\frac{2}{3} x+10 A$ \\
\hline
\end{tabular}


o primeiro bloco já está diagonalizado e corresponde a um estado duplamente degenerado com energia

$$
E_{11}=E_{12}=E_{0}+W(-2 A+2 X)
$$

onde Eo é a energia do estado ${ }^{4} F_{3}=$ para 0 lon livre. Os autoestados correspondentes a este primeiro bloco săo:

$$
\begin{aligned}
& v_{11}=|3 / 2\rangle \\
& v_{12}=|-3 / 2\rangle
\end{aligned}
$$

Os outros dois blocos são do tipo:

$$
\left(\begin{array}{ll}
a & b \\
b & c
\end{array}\right)
$$

Esta matriz tem como auto-valores

$$
\lambda_{1,2}=\frac{(a+c) \pm \sqrt{(a-c)^{2}-4 b^{2}}}{2}
$$

e auto-vetores

$$
\begin{aligned}
& u_{1}=\zeta_{1}\left(\begin{array}{c}
\left.\frac{b}{\left(a-\lambda_{1}\right.}\right) \\
1
\end{array}\right) \\
& u_{2}=\zeta_{2}\left(\begin{array}{c}
\left.\frac{b}{\left(a-\lambda_{2}\right.}\right) \\
1
\end{array}\right)
\end{aligned}
$$

Desta forma os blocos 2 e 3 da matriz principal resultam nos mesmos auto-valores com auto-vetores diferentes, ou seja, no final da diagonalizaçăo dos blocos 2 e 3 teremos apenas dois estados duplamente degenerados. 
Primeiramente tomemos obloco 2 . Usando a diagonalização anterior temos que

$$
\begin{aligned}
& E_{z_{1}}=E_{0}+W\left[(A-x)+3 \sqrt{x^{2}+\frac{2}{3} A x+9 A^{2}}\right] \\
& E_{z=}=E_{0}+W\left[(A-x)-3 \sqrt{x^{2}+\frac{2}{3} A x+9 A^{2}}\right]
\end{aligned}
$$

com os seguintes auto-estados

$$
\begin{aligned}
& v_{21}=K_{1}(x)|5 / 2\rangle+K_{2}|-1 / 2\rangle \\
& \left.v_{x=2}=K_{1} \cdot(x) 15 / 2\right\rangle+K_{2} \cdot|-1 / 2\rangle
\end{aligned}
$$

Procedendo de forma análoga, vemos que o bloco três tem os mesmos auto-valores, mas com os seguintes auto-estados :

$$
\begin{aligned}
& v_{=1}=K_{1}(x)|1 / 2\rangle+K_{2}(x)|-5 / 2\rangle \\
& \left.v=K_{1}^{\prime}(x)|1 / 2\rangle+K_{2} \cdot(x) 1-5 / 2\right\rangle .
\end{aligned}
$$

Portanto os estados $v=1$ e $v=1$ formam um estado $e$ os estados $V=a$ e $V=z$ formam o outro.

Atraves das medidas experimentais e do estudo feito com a teoria de grupos chegamos as seguintes tres energias que supomos serem relativas aos niveis do estado ${ }^{4} \mathrm{~F}=12$ do $\mathrm{Er}^{+3}$ no $\mathrm{LiNbO}=$ :

$$
\begin{aligned}
& E_{1}=22055.6 \mathrm{~cm}^{-1} \\
& E_{0}=22016.7 \mathrm{~cm}^{-1} \\
& E_{-}=21997.4 \mathrm{~cm}^{-1} .
\end{aligned}
$$

Através das equaçres (71) e (76) temos que :

$$
\begin{aligned}
& 22055.6-E_{0}=\hat{-E_{1}} \\
& 22016.7-E_{0}=-E_{2} \\
& 21997.4-E_{0}=\hat{-} E_{2}
\end{aligned}
$$

com

$$
\hat{A} E_{1}+\hat{-} E_{2}+\hat{A} E_{5}=0
$$


desta forma vemos que o valor de $E_{0}=22023.2 \mathrm{~cm}^{-1}$ e que temos efetivamente apenas duas equações linearmente independentes . Este resultado nos leva a trabalhar com as incognitas WX e WA para que o sistema de equaçóes seja soluvel.

Calculando os $\Delta E_{x}$ calmos no seguinte sistema

$$
\begin{gathered}
-2(W A)+2(W x)=-6,5 \\
(W A)-(W x)-3 \sqrt{(W x)^{2}+\frac{2}{3}(W A)+9(W A)^{2}}=-25,8 \\
(W A)-(W x)+3 \sqrt{(W x)^{2}+\frac{2}{3}(W A)+9(W A)^{2}}=32,4
\end{gathered}
$$

Tomando qualquer par de equaçðes chegamos ao seguinte resultado:

$$
\begin{aligned}
& (W A)=3.239 \\
& (W x)=-0.011 .
\end{aligned}
$$

portanto o sistema é solúvel com as energias apresentadas e conseguimos determinar com estes valores todas as constantes da Hamiltoneana do problema.

Para testar os resultados obtidos podemos fazer $A=0$ e observar se o resultado é o mesmo que o previsto para o campo cúbico. Na figura 58 temos o diagrama de niveis para os dois $\operatorname{casos} W A=0$ e WA $=3,239$.

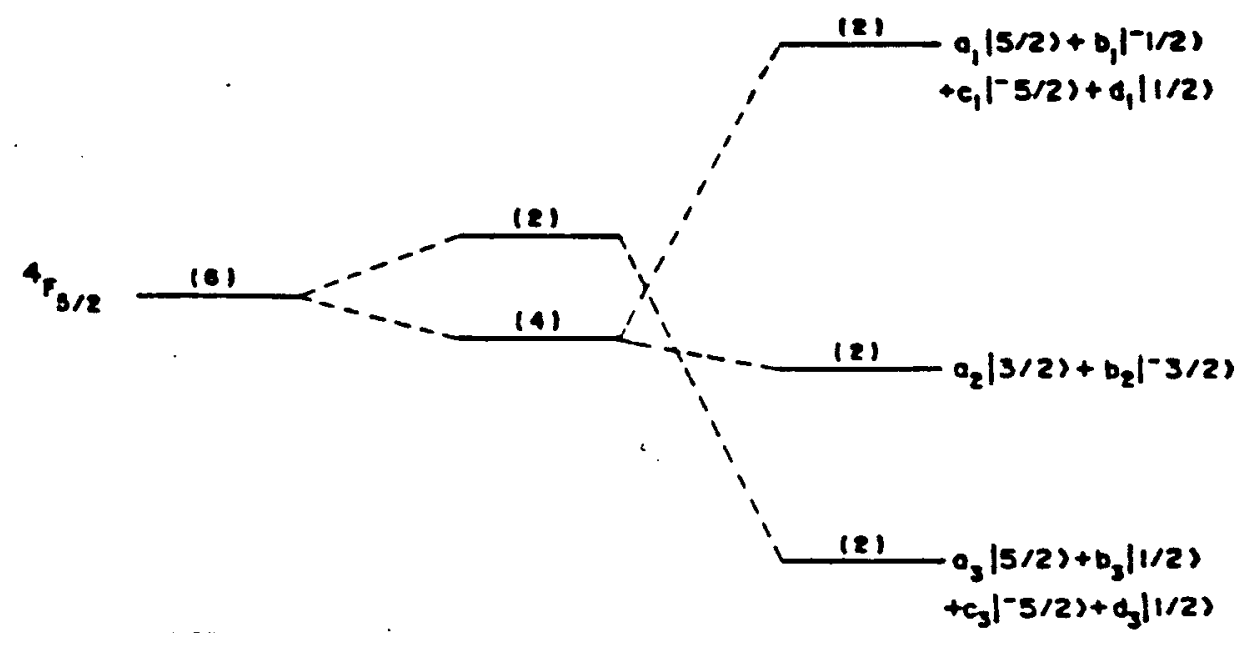

\footnotetext{
LIVRE On $c_{3}$

figura 58 - Desdobramento dos niveis para um estado com $J=5 / 2$ para simetrias on e $C_{3}$.
} 
No caso de $J$ com valores maiores que $5 / 2$ as matrizes relativas a perturbaçăo do campo cristalino văo ficando mais complexas e nem mesmo a divisão em blocos facilita o problema pois. estes blocos são de dimensões altas $(3.4 \ldots)$ e isto vai dificultando cada vez mais o trabalho da idenficação dos niveis de energia. Neste ponto fica mais facil utilizar um programa de diagolização de matrizes, e tambem para $J>5 / 2$ ja se começa a ter informaçoes do valor de $x$. Desta forma fica apenas o valor de $A$ para ser ajustado pois oW, como é uma constante que multiplica toda a matriz, pode ser momentaneamente excluido do problema se trabalharmos nao com os valores absolutos dos $\hat{A}_{1}$ mas sim com a razăo entre eles.

A matriz para $\mathrm{J}=7 / 2$ está montada a seguir.

$: 7 / 2\rangle \quad: 5 / 2\rangle \quad: 3 / 2\rangle \quad: 1 / 2\rangle \quad:-1 / 2\rangle \quad:-3 / 2\rangle \quad:-5 / 2\rangle \quad:-7 / 2\rangle$

\begin{tabular}{|c|c|c|c|c|c|c|c|c|}
\hline & & & & & & & & \\
\hline $17 / 2\rangle$ & $\mathrm{A}$ & 0 & 0 & $B$ & 0 & 0 & $C$ & 0 \\
\hline$\{5 / 2\rangle$ & 0 & $\mathrm{D}$ & 0 & 0 & $E$ & 0 & 0 & $c$ \\
\hline$: 3 / 2\rangle$ & 0 & 0 & $F$ & 0 & 0 & 0 & 0 & 0 \\
\hline$: 1 / 2\rangle$ & B & 0 & 0 & $G$ & 0 & 0 & $-E$ & 0 \\
\hline$i-1 / 2\rangle$ & 0 & $E$ & 0 & 0 & G & 0 & 0 & -8 \\
\hline$:-3 / 2>$ & 0 & 0 & 0 & 0 & 0 & $F$ & 0 & 0 \\
\hline$:-5 / 2\rangle$ & $c$ & 0 & 0 & $-E$ & 0 & 0 & D & 0 \\
\hline$|-7 / 2\rangle$ & 0 & $C$ & 0 & 0 & $-B$ & 0 & 0 & A \\
\hline
\end{tabular}


onde

$$
\begin{aligned}
& A=-\frac{14}{3} x+\frac{16}{9}(1-x)+21 A \\
& B=\sqrt{70} \frac{4}{3} x+\frac{8}{9}(1-x) \\
& C=\frac{44}{9} \sqrt{7}(1-x) \\
& D=\frac{26}{3} x-\frac{80}{9}(1-x)+3 A \\
& E=\sqrt{10} \frac{8}{3} x-\frac{28}{9}(1-x) \\
& F=2 x+16(1-x)-9 A \\
& G=-6 x-\frac{80}{9}(1-x)-15 A
\end{aligned}
$$

Os sinais de $w$ e $x$ podem ser determinados atraves das constantes $\mathrm{B}_{4}$ e $\mathrm{B}_{6}$. Pela equaça vemos que $O$ sinal de $W$ é determinado por $B_{6}$, pois $F(\sigma)$ e uma constante positiva. O sinal de $x$, como mostra a equação (67), pode ser determinado pela razão $\left(B_{4} / B_{s}\right)$. As constantes $B_{4}$ e $B_{s}$ são proporcionais as constantes $\beta_{4}$ e $\gamma$, . respectivamente. Em geral, o calcuio das constantes $\beta_{4}$ e $\gamma_{*}$ nåo é muito trivial, exceto para 0 fundamental cujos valores se encontram até tabelados. Entretanto, para um determinado multipleto vale a seguinte relaçă :

$$
\begin{aligned}
& B_{J}=(-1)^{J} J^{\prime} f\left(\text { LSJJ') } \beta_{J}\right. \\
& \gamma_{J}=(-1)^{J-J} f^{\prime}\left(\text { LSJJ') } \gamma_{J}\right.
\end{aligned}
$$

onde f e uma função positiva. Portanto conhecendo-se os valores de $\beta_{,}$e $\gamma$, do fundamental pode-se obter 0 sinal destas constantes para os estados excitados relativos a seu multipleto. Para o erbio trivalente temos

$$
\begin{aligned}
& \beta_{3}=0.44410^{4} \\
& \gamma_{3}=2.069910^{5} .
\end{aligned}
$$


Para os demais estados excitados informaçðes deste tipo são mais complicadas para se obter. No nosso caso os valores de $x e$ - sinal de $W$ foram obtidos atraves da inspecão nos graficos da figura 57 .

No caso do estado ${ }^{4} \mathrm{~F}_{>1 z}$ conseguimos um erro de $0,01 \%$ na determinaçăo dos niveis de energia quando diagonalizamos a matriz $\mathrm{J}=7 / 2$ com $\mathrm{x}=0.0115$ e $\mathrm{A}=-0.206$ e multiplicamos os resultados por $W=4,9785 \mathrm{~cm}^{-1}$. Na tabela 25 temos uma comparaçăo entre os resultados experimentais e os calculados por este método. Nesta tabela pode-se observar que no caso do nivel ${ }^{4} F_{7 / 2}$ o erro de $0.01 \%$ na energia refletiu num erro de $0 \%$ no comprimento de onda na aproximação da primeira casa decimal, que e a ordem de precisao oferecida pela aparelhagem experimental.

Na tabela 24 temos os valores de $x$, A e W obtidos para os demais estados do $\mathrm{Er}^{+3}$.

$\mathrm{Na}$ tabela 25 temos uma comparação entre os resultados experimentais e os calculados mostrando que a identificão dos niveis de energia foi bem sucedida.

TABELA 24 - Valores de $x$, A e W para alguns estados do Er+I

\begin{tabular}{|c|c|c|c|}
\hline ESTADO & $x$ & A & $\mathrm{W}\left(\mathrm{cm}^{-1}\right)$ \\
\hline${ }^{4} I_{01}=$ & 0.7000 & 0.900 & -2.8000 \\
\hline${ }^{4} F_{9 / 2}$ & 0,7140 & 0.299 & -1.4000 \\
\hline$=\mathrm{H}_{11}=\mathrm{z}$ & 0.8000 & -0.600 & 1.2000 \\
\hline${ }^{4} F_{>}=$ & 0.0115 & -0.206 & 4.9785 \\
\hline$=\mathrm{Ho/2}$ & 0.6400 & 1.000 & -3.4000 \\
\hline${ }^{4} G_{12} /=$ & 0.9700 & 1.500 & 1.6000 \\
\hline
\end{tabular}


TABELA 25 - Comparaçåo entre os resultados experimentais e os calculados.

\begin{tabular}{|c|c|c|c|}
\hline NIVEL & REP. IRR. & $\lambda \equiv \times p(\mathrm{~nm})$ & $\lambda_{\text {CAL }}(\mathrm{nm})$ \\
\hline \multirow{5}{*}{${ }^{4} I_{9 / 2}=$} & $\Gamma_{0}$ & 793.0 & 793.1 \\
\hline & $\Gamma_{4 .}=$ & 796.9 & 796.7 \\
\hline & $\Gamma_{4,}=$ & 803,0 & 803.1 \\
\hline & $\Gamma s$ & 807.0 & 806.2 \\
\hline & $\Gamma_{4,}=$ & 807,7 & 808.5 \\
\hline \multirow{5}{*}{${ }^{4} F_{91}=$} & $\Gamma_{4 .}=$ & 651.4 & 651.5 \\
\hline & $\Gamma_{\phi}$ & 651.9 & 652.0 \\
\hline & $\Gamma_{4}=$ & 654.2 & 654,2 \\
\hline & $\Gamma_{s}$ & 655.0 & 655.1 \\
\hline & $\Gamma_{4 .}=$ & 655.5 & 655.3 \\
\hline \multirow{2}{*}{${ }^{4} S_{x / 2}$} & $\Gamma_{4 .}=$ & 545.2 & 545.2 \\
\hline & $\Gamma_{s}$ & 547.9 & 547.9 \\
\hline \multirow{6}{*}{$=H_{11 / 2}$} & $\Gamma_{4,}=$ & 521.0 & 521.1 \\
\hline & $\Gamma_{4}=$ & 521.4 & 521.7 \\
\hline & $\Gamma_{\omega}$ & 522,1 & 521.7 \\
\hline & $\Gamma_{s}$ & 524.7 & 524.9 \\
\hline & $\Gamma_{4}=$ & 525.3 & 525.2 \\
\hline & $\Gamma_{4}=$ & 526.4 & 526.2 \\
\hline \multirow{4}{*}{${ }^{4} F_{712}$} & $\Gamma_{4}=$ & 486.5 & 486.5 \\
\hline & Гs & 486.9 & 486.9 \\
\hline & $\Gamma_{4 .}=$ & 490.0 & 490.0 \\
\hline & $\Gamma_{4 .}=$ & 491.0 & 491.0 \\
\hline
\end{tabular}


continuaçåo

\begin{tabular}{|c|c|c|c|}
\hline NIVEL & REPR . IRR. & $\lambda \equiv \times F(n m)$ & $\lambda_{\text {CAL }}(\mathrm{nm})$ \\
\hline \multirow{3}{*}{${ }^{4} \mathrm{~F}=12$} & $\Gamma_{4 .}=$ & 453.0 & 453.0 \\
\hline & $\Gamma_{s}$ & 453.4 & 453.4 \\
\hline & $\Gamma_{4 n}=$ & 454.6 & 454.6 \\
\hline \multirow{2}{*}{$4 F_{31=}$} & $\Gamma_{4,}=$ & 445,2 & 445.2 \\
\hline & $\Gamma_{\omega}$ & 447.7 & 447.7 \\
\hline \multirow{5}{*}{$=\mathrm{H}_{\infty}=2$} & $\Gamma_{s}$ & 406.8 & 406.5 \\
\hline & $\Gamma_{4 .}=$ & 407.5 & 407.7 \\
\hline & $\Gamma_{4,}=$ & 409.5 & 409.5 \\
\hline & $\Gamma_{\omega}$ & 410.5 & 410.8 \\
\hline & $\Gamma_{4}=$ & 411.8 & 411.7 \\
\hline \multirow{6}{*}{$4 G_{11}=$} & $\Gamma_{s}$ & 379.0 & 379.1 \\
\hline & $\Gamma_{4,}=$ & 379.4 & 379.4 \\
\hline & $\Gamma_{4 n}=$ & 379.7 & 379.6 \\
\hline & $\Gamma_{0}$ & 381,8 & 381.8 \\
\hline & $\Gamma_{4 .}=$ & 383.0 & 382.7 \\
\hline & $\Gamma_{4}=$ & 383,8 & 384.1 \\
\hline
\end{tabular}

\subsection{ANÁLISE DOS ESPECTROS DE EMISSAO}

Quando bombeamos o cristal LiNbos:Er+s com a linha $488 \mathrm{~nm}$ do laser de argorio estamos excitando o nivel ${ }^{4} F_{7}=$ do Ert. Na figura 59 temos uma diagrama mostrando quais são, a principio. as energias de emissăo esperadas. 


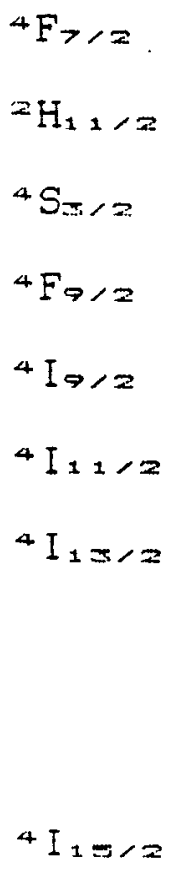

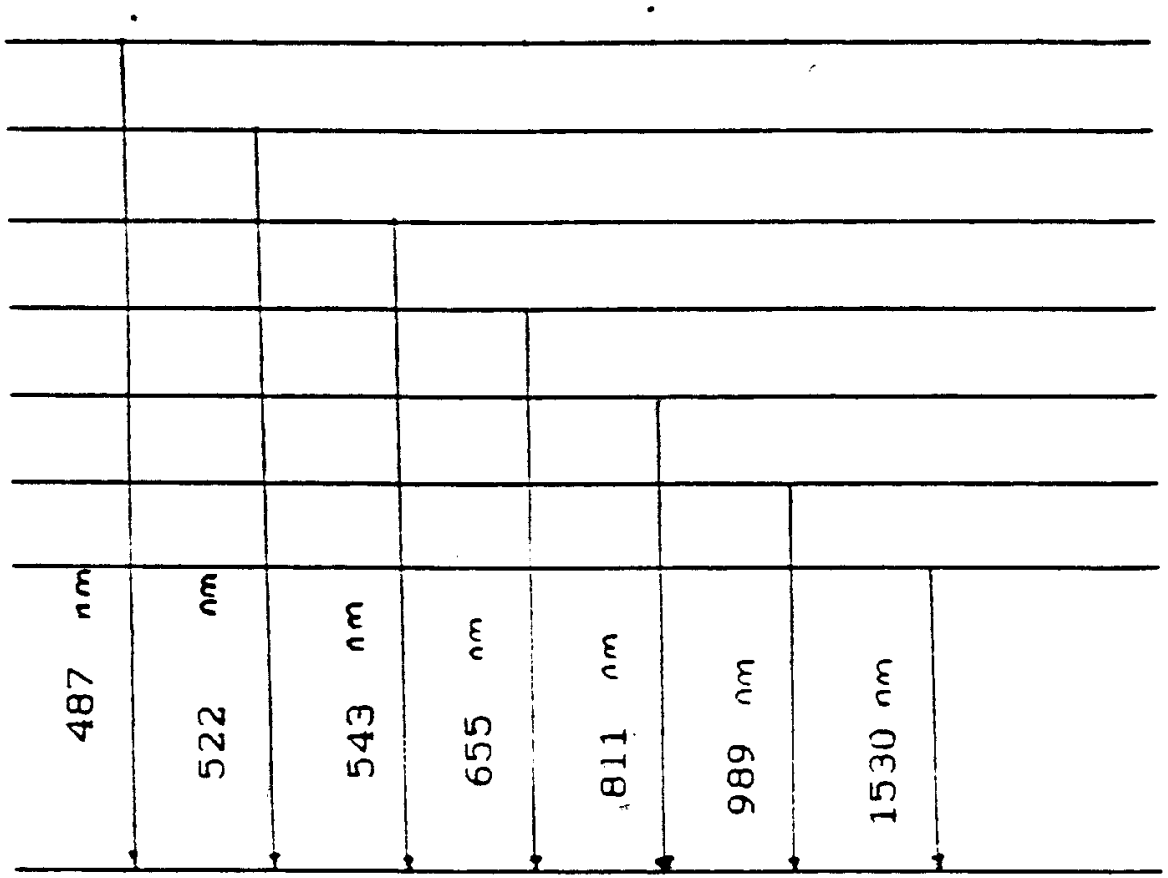

$20524 \mathrm{~cm}^{-1}$

$19166 \mathrm{~cm}^{-1}$

$18432 \mathrm{~cm}^{-1}$

$15266 \mathrm{~cm}^{-1}$

$12328 \mathrm{~cm}^{-1}$

$.10123 \mathrm{~cm}^{-1}$

$6540 \mathrm{~cm}^{-1} \mathrm{O}$

figura 59 - Emissões possiveis no sistema LiNbOs:Er+: quando se bombeia o sistema com laser de argônio.

Observando os resultados experimentais vemos que observamos as transições $2 \mathrm{H}_{11 / z \rightarrow}{ }^{4} \mathrm{I}_{1=/ 2},{ }^{4} \mathrm{~S}_{1 / 2} \rightarrow{ }^{4} \mathrm{I}_{1=1 / 2} \mathrm{e}$ ${ }^{4} \mathrm{~F}_{9 / 2} \rightarrow{ }^{4} \mathrm{I}_{1=/ 2}$.

Identificada as transições podemos analisar o comportamento de cada uma separadamente. Vamos iniciar com a transição $=\mathrm{H}_{11 / 2 \rightarrow} \rightarrow{ }^{4} \mathrm{I}_{1=/ 2}$.

Esta transição possui uma particularidade muito interessante . Quando abaixamos a temperatura ela desaparece totalmente. A principio podemos supor que este efeito esteja relacionado com inversao térmica de populaçăo, ou seja. - sistema quando decal do estado ${ }^{4} F_{7}=$ vai direto para o estado ${ }^{4} S_{3} r=$ e depois e excitado termicamente para o estado ${ }^{2} \mathrm{H}_{1+12}$. Um cálculo grosseiro pode ser feito supondo que o processo ocorra com tempo suriciente para se ter um equilibrio térmico, neste caso temos : 
onde $N_{1}$ é a população do nivel ${ }^{4} S_{x}=, N_{2}$ é a população do nivel $=\mathrm{H}_{11, z}$, $\quad \hat{E}$ é diferença de energia entre os dois niveis, $k$ é a constante de Boltzmann e $T$ e a temperatura em graus Kelvin. Sendo a diferença de energia entre os estados da ordem de $750 \mathrm{~cm}^{-1}$ e estando a temperatura ambiente temos a seguinte relaçå entre a populaçăo dos dois niveis :

$$
\mathrm{N}_{2} / \mathrm{N}_{1} \approx 0,1
$$

Abaixando a temperatura para $77 \mathrm{~K}$ temos

$$
N_{2} / N_{1} \approx 310^{-\infty}
$$

Como se pode ver a população cai significativamente quando abaixamos a temperatura. Este fato tambem é coerente com o aumento da intensidade da transiçăo ${ }^{4} S_{3 / 2} \rightarrow{ }^{4} I_{1} / 2$, , ou seja, quando abaixamos a temperatura praticamente toda a população que decai do estado ${ }^{4} F_{7}=$ fica em ${ }^{4} S_{3}=$ e para depois decair para 0 fundamental.

A transiçăo ${ }^{4} S_{3}=-{ }^{4} I_{1=1 z}$ é a mais intensa das très transiçชes observadas. Atraves dela, dos estudos feitos pelo espectro de absorção e da teoria de grupos pudemos identificar razoavelmente os niveis do fundamental. Na tabela 26 temos os resultados obtidos.

Os valores das energias săo razoavelmente compatıveis com os resultados obtidos por Gabrielyan e Kaminskiile, entretanto, a identificaçăo das representaçoes irredutıveis, nå se pode comparar pois eles nao fizeram o estudo do espectro polarizado. 
TABELA 26 - Energias do estado fundamental do Er+ em LiNbOs

\begin{tabular}{lc}
\hline Repr. Irr. & Energia $\left(\mathrm{cm}^{-1}\right)$ \\
\hline$\Gamma_{4}=$ & 0 \\
$\Gamma_{4,=}$ & 63 \\
$\Gamma_{\infty}$ & 129 \\
$\Gamma_{4}=$ & 152 \\
$\Gamma_{\infty}$ & 185 \\
$\Gamma_{4}=$ & 269 \\
$\Gamma_{4}=$ & 353 \\
$\Gamma_{\infty}$ & 414 \\
\hline
\end{tabular}

Baseados nos niveis de energia obtidos para o espectro de absorção para os estados ${ }^{4} \mathrm{~F}_{9 i z}$ e ${ }^{4} \mathrm{~S} \Omega / \approx$ resultados obtidos através da transição ${ }^{4} S_{3 / 2} \rightarrow{ }^{4} I_{1=12}$ para 0 estado fundamental, temos as seguintes previsões para o espectro de emissão :

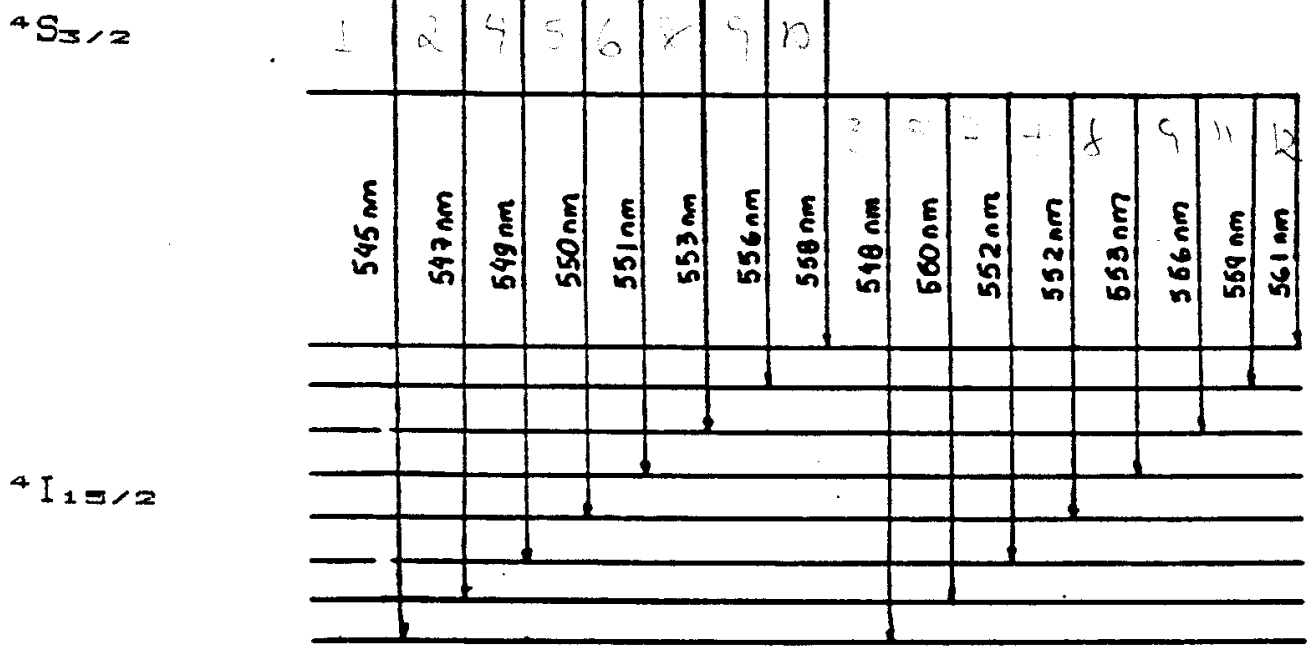

$18342 \mathrm{~cm}^{-1}$

$18252 \mathrm{~cm}^{-1}$

$414 \mathrm{~cm}^{-1}$

$353 \mathrm{~cm}^{-1}$

$269 \mathrm{~cm}^{-1}$

$185 \mathrm{~cm}^{-1}$

$152 \mathrm{~cm}^{-1}$

$129 \mathrm{~cm}^{-1}$

$63 \mathrm{~cm}^{-1}$

$0 \mathrm{~cm}^{-1}$

figura 60 - Transiçסes possiveis entre os niveis ${ }^{4} S_{3 / z} \rightarrow{ }^{4} I_{13 / z}$. 


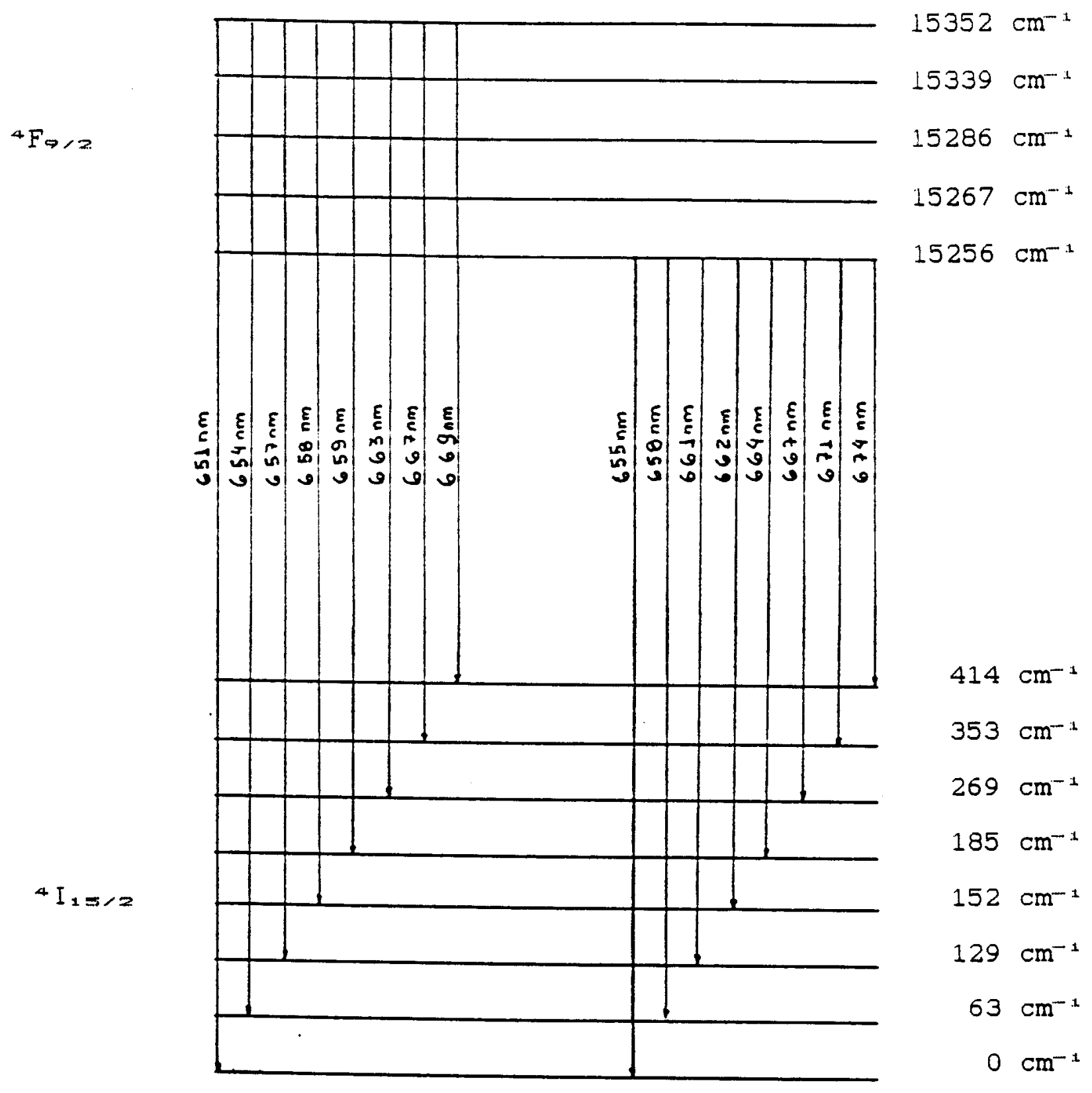

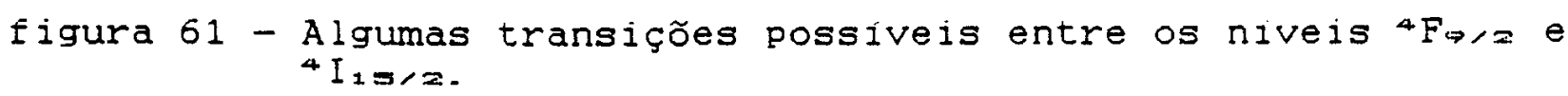

Como se pode ver pelas figuras 00 e 61 existe uma boa compatibilidade entre nossos resultados de absorça e emissao . Este fato mostra que os resultados obtidos săo bons. 


\subsection{ANÁLISE DAS MEDIDAS DE TEMPO DE VIDA LUMINESCÊNCIA}

Dentro da resoluçăo do aparato experimental conseguimos ter uma ideia do valor aproximado dos tempos de vida das transiçós

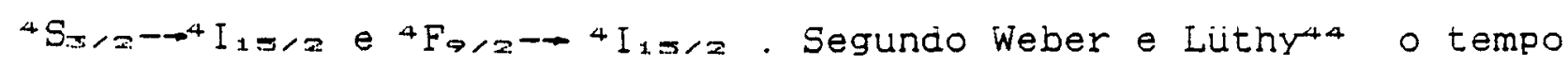

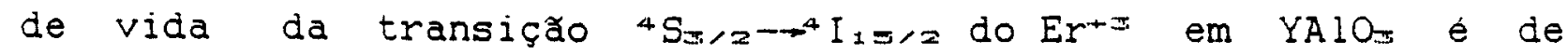
aproximadamente 117 us a temperatura ambiente. No nosso caso medimos 60 us para temperatura ambiente e 120 us para temperatura de nitrogenio liquido. Comparando estes resultados vemos que estăo dentro da mesma ordem de grandeza, como deveria ser . A diferença entre os valores se deve a rede cristalina, pois o tempo de vida de luminescência de uma transiçăo vai depender do tempo de vida do estado e do tempo de decaimento de outros processos envolvidos no processo da luminescencia, os quais eståo fortemente relacionados com a rede. 


\section{VI . CONCLUSOES}

Saber controlar as concentrações de $\mathrm{OH}^{-}$e $\mathrm{Fe}^{+2}$ em LiNbOs:EY é extremamente importante quando se visa a utilização deste cristal como meio ativo para laser de estado solido. pois - $\mathrm{Fe}^{+z}$ é reponsavel pelo efeito fotorefrativo deste cristal e o $\mathrm{OH}^{-}$absorve em 2.7 um que é justamente a regiăo onde o $\mathrm{Er}^{+}$, em outras matrizes, possui emissao laser.

Neste trabalho conclui-se que uma solução para estes problemas é obtida via tratamentos térmicos. O tratamento mais rapido, mais eficiente $e$ que menos influencia nas propriedades do cristal é o realizado a $700^{\circ} \mathrm{C}$ numa atmosfera de oxigênio durante um periodo de oito horas.

Em relaçao a caracterizaçao do cristal como meio ativo para laser conseguiu-se chegar a alguns pontos importantes : determinaram-se os niveis do erbio nesta matriz ( ver fig 62 ), observou-se $\circ$ comportamento de suas transiçбes no que diz respeito a polarizaça e mediu-se $\circ$ tempo de vida das transiçơes.

Para ajudar na determinação dos niveis de energia utilizaram-se cálculos teoricos. Estes calculos envolveram conceitos de teoria de grupos e teoria de campo ligante. Os resultados obtidos foram bastante satisfatorios. pois o erro entre os valores de comprimento de onda medidos e calculados foi de aproximadamente $0.03 \%$.

- comportamento dicróico das transiçбes neste cristal é bastante acentuado. Com as informaçbes oferecidas pela teoria de 
grupos foi possivel identificar cada nivel com sua respectiva representaçăo irredutivel e conseguentemente deixar definida a polarizaçåo de cada transiçao.

Os estados ${ }^{4} \mathrm{~S} z /=$ e ${ }^{4} \mathrm{~F}_{\nexists \gamma z}$ são conhecidos por serem metaestaveis em outras matrizes e consequentemente candidatos a possuirem transiçбes laser. Neste trabalho tambem observou-se um tempo de vida relativamente longo, da ordem de $0.05-0.1 \mathrm{~ms}$. nas transiçôes destes estados para o fundamental.

Atraves destes resultados pode-se tambem concluir que, embora não se consiga afirmar com plena certeza que o sistema LiNbOs:Er-m seja um meio ativo para laser nas transiçoes ${ }^{4} S_{312} \rightarrow{ }^{4} I_{1=12}$ e ${ }^{4} F_{9 r z} \rightarrow{ }^{4} I_{1=1 z}$, este trabalho mostra que e viável e deve-se continuar pesquisando no sentido de medir a potência de "threshold" das transiçðes apresentadas e de outras que possam existir, principalmente na regiao do infra-vermelho, assim como desenvolver o laser de niobato de litio.

O interesse neste cristal para matriz de lons laser se deve ao fato dele possuir inúmeras propriedades interessantes e que podem ser aplicadas para desenvolver dipositivos integrados oticos . como por exemplo: laser com "Q-swich"interno e laser auto-dobrador de frequencia.

Paralelamente a este trabalho tambem estao sendo realizadas pesquisas dentro do grupo nas areas de construção da cavidade para LiNbos:TR e transferencia de energia entre $\mathrm{Cr}^{+3}$ e $\mathrm{Er}^{+3}$ em cristais duplamente dopados. 


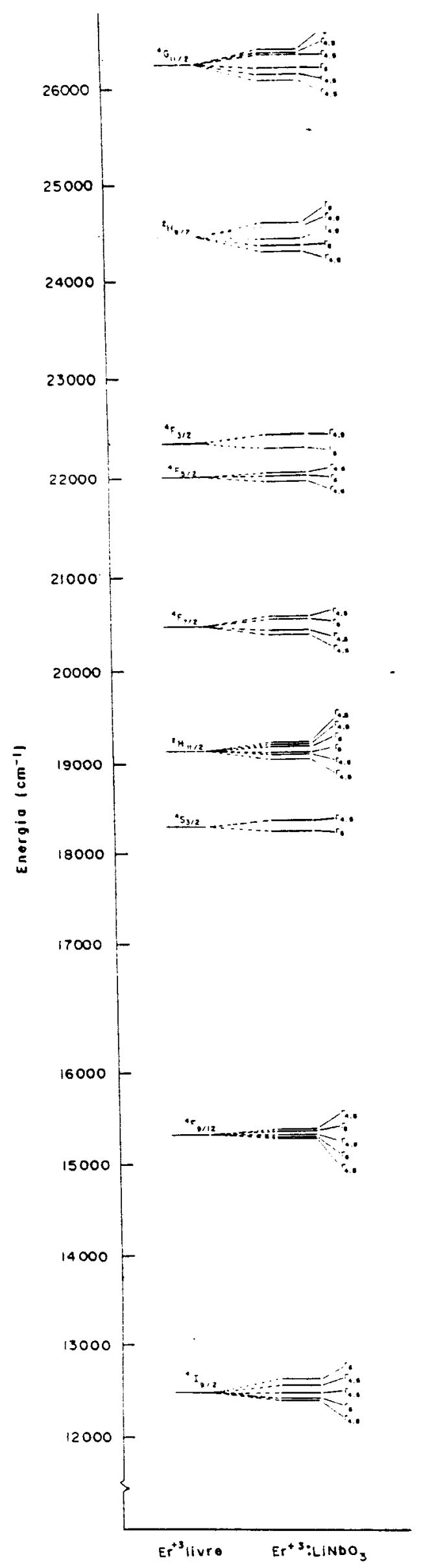




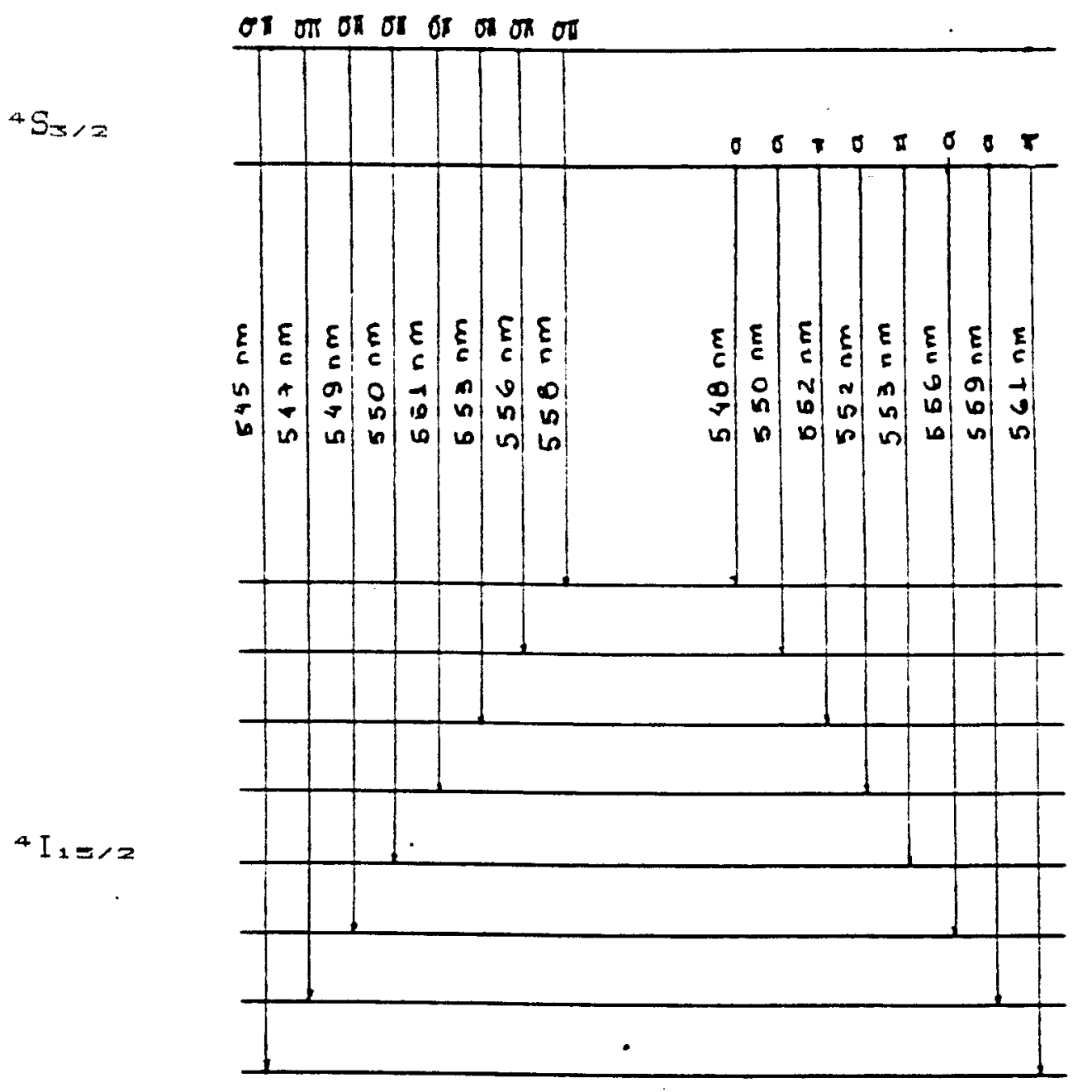

$18342 \mathrm{~cm}^{-1}$

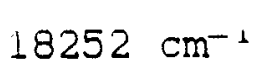

$414 \mathrm{~cm}^{-1}$

$353 \mathrm{~cm}^{-1}$

$269 \mathrm{~cm}^{-1}$

$185 \mathrm{~cm}^{-1}$

$152 \mathrm{~cm}^{-1}$

$129 \mathrm{~cm}^{-1}$

$63 \mathrm{~cm}^{-1}$

$0 \mathrm{~cm}^{-1}$

figura 63 - Transiçðes entre os nıveis ${ }^{4} S_{31}=e^{4} I_{15 / 2}$ 


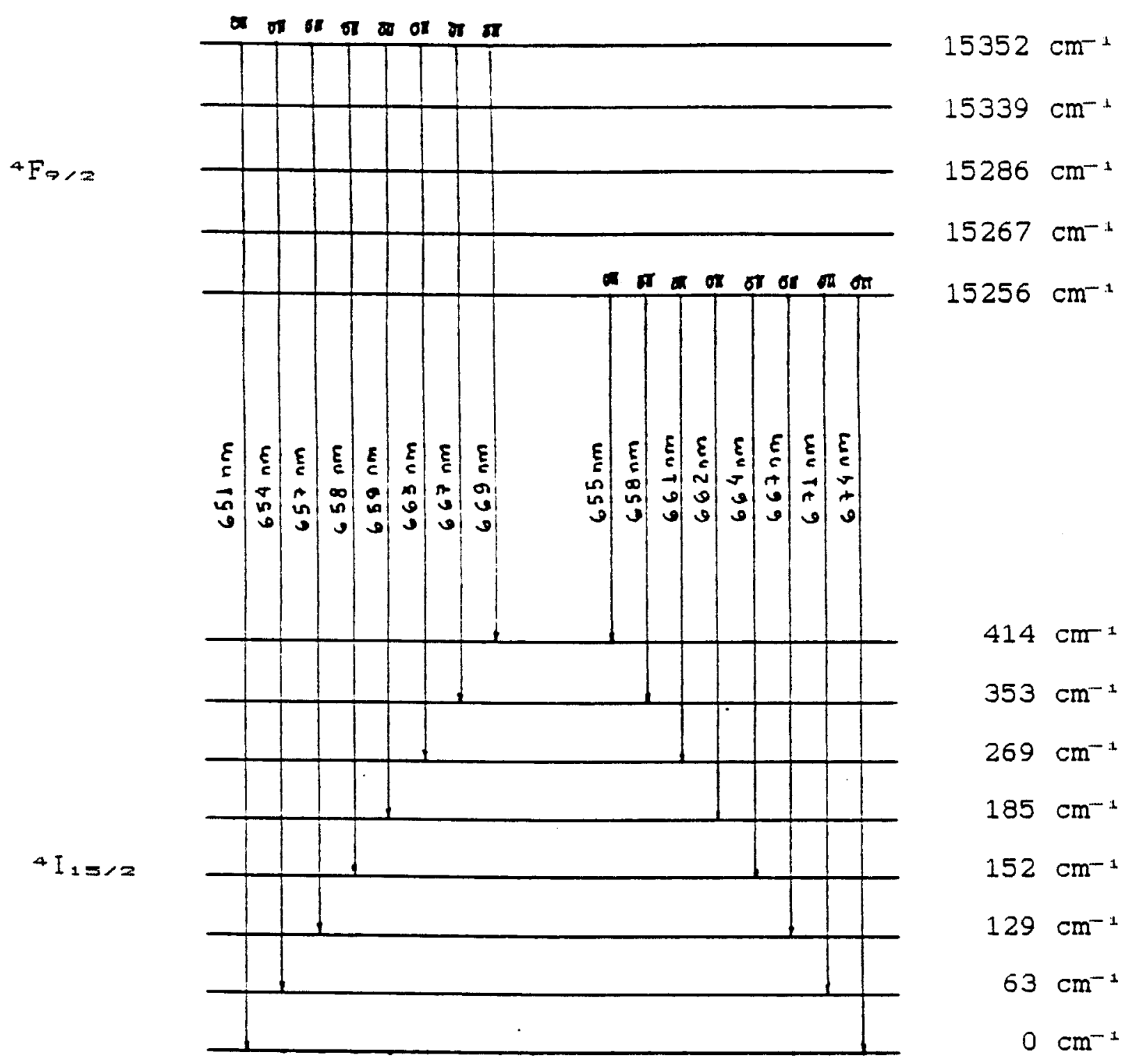

figura 64 - Algumas transições possiveis entre os niveis ${ }^{4} \mathrm{~F}_{912}$ e ${ }^{4} I_{1=12}$ 


\section{VII - BIBLIOGRAFIA}

1 ABRAGAM, A. and BLEANEAY. B., Electron Paramagnetic Resonance of Transition Ions, Oxford University Press, London. 1969.

2 ABRAHAMS, S.C.; REDDY, J.M. and BERNSTEIN. J.L.. J. Phys. Chem. Solids, 27,997 (1966).

3 ANDREETA, J.P. e OCTAVIANO, E.S. , Rev. Fis. Apl. Instrum., 3. $30(1988)$.

4 ARIZMENDI, L. and CABRERA, J.M., PhYs. Rev. B, 31 , 7138 (1985).

5 ARIZMENDI, L.; ABELLA, F. and CABRERA, J.M., Ferroeletrics. 56,75 (1984).

6 ARIZMENDI, L.; CABRERA, J.M. and AGULLO-LOPEZ, F... J. Phys. C. $17,515(1984)$.

7 BAGNATO, V.S., Espectroscopia Infraverme lho e RAMAN do GdAlO= Puro e Dopado com Ions Terras Raras, Dissertação de Mestrado, DFCM-IFQSC, Sao Carlos, 1983.

8 BALLHAUSEN, C.J., Introduction to Ligand Field Theory, McGraw-Hill Book Company, 1962.

9 BELABAEV, K.G.; KAMINSKII, A.A. and SARKISOV, S.E., Phys. Stat. Sol. (A) $28, \mathrm{~K} 17$ (1975). 
10 ClARCK, M.g.; Di SALVo, F.J.; GLASS, A.M. and PETERSON, G.E., J. Chem. Phys. , 59, 6209 (1973).

11 CORDOVA-PLAZA, A.; DIGONNET, M.J.F. and SHAW.H.J., IEEE J. Quantum Electronics, QE-23, 262 (1987).

12 DÄTWYLER, M.; LUUTHY, W. and WEBER, H.P., IEEE J.Quantum Eletronics, QE-23, 158 (1987).

13 DeSHAZER, L.G., Laser Focus/Electro-Optics, 23,158 (1987).

14 DEXTER, D.L. . Theory of Optical Properties of Imperfections in Nonmetals, em Solid State Physics, vol. 6 , editado por F.Seitz e D.Turnbul1, Academic, New York, p.353 (1958).

15 DI BARTOLO, B., Optical Interations in Solids, John wiley and Sons inc., 1968.

16 ELIIOTT, J.P.; JUDD, B.R. and RUNCIMAN, W.A., Proc. Roy. Soc., A240, 509 (1957).

17 EVLANOVA, N.L.; KOVALEV, A.S.; KOPTSIK, V.A.; KORNIENKO, L.S.: PROKHOROV, A.M. and RASHKOVICH. L.N., JETP Letters, 5.291 (1967).

18 GRABIELYAN, V.T.; KAMINSKII, A.A. and LI, L., Phys. Stat. Sol. (a). 3, K37 (1970).

19 HERRINGTON, J.R.; DISCHLER, B.; RAUBER. A. and SCNEIDER, J.. Sol. Stat. Commun., 12,351 (1973).

20 HOLMES, R.J.; Kim. V.S.; BRANDLE, C.D. and SMITH, D.M., Ferroeletrics, 51,41 (1983).

21 HUTCHINGS, M.T. Point-Charge Calculations of Energy Levels of Magnetic Ions in Crystaline Electric Field. 
22 JOHNSON, L.F. and BALLMAN, A.A., J. Appl. Phys., 40, 297 (1969).

23 KAMINOW, I,P, and STULZ, I.W., IEEE J. Quantum Eletronics, 11.306 (1975).

24 KAMINSKII, A.A., Sov. Phys. Dokl, 27 (12), 1039 (1982).

25 KAMINSKII, A.A., Laser Crystals, Springer-Verlag, Berlin Heidelberg and New York, 1981.

26 KAMISKII, A.A.; FEDOROV, V.A.: IANOV, A.O.; MOCHALOV, I.V. and KRUTOVA. L.I., Sov. Phys. Dokl, 27 (9), 725 (1982).

27 KETCHHUM, J.L.; SWEENEY, K.L.; HALLIBURTON, L.E. and ARMINGTON, A.F.. Phys. Lett. A94, 450 (1983).

28 KOECHNER, W.. Solid State Laser Engineering, Springer Series in Optical Sciences, 1976

29 KOSTER, G.F.; DIMNOCH, J.O.; WHEELER, R.G. and STATZ, H. . Properties of the thirty-two point groups. MIT Press. Cambridge, Massachusetts, 1963.

30 KRUPKE, W.F.; and GRUBER, J.B., The Journal of Chemical Physics, 39,1024 (1963).

31 LEA, K.R.; LEASK, M.J.M. and WOLF, W.P., J. PhYs. Chem. Solids, 23,1381 (1962).

32 MASTELARO, V.R., Caracterização de Monocristais de Niobato de Litio ( $\mathrm{LiNbO}$ ) Dopados com Oxido de Cromo ( $\mathrm{CrO}=$ ) e com Oxido de Ferro ( $\left.\mathrm{Fe}_{z} \mathrm{O}_{3}\right)$, Dissertação de Mestrado. DFCMIFQSC, Sao Carlos. 1988. 
33 NASSAU, K., Lithium niobate - a new type of ferroeletric: Growth, structure and properties, Ferroelectricity, E.F. Weller, ed. (Elsevier. Amsterdam, 1967).

34 PETROV. M.V. and TKACHUK, A.M., Opt. Spektrosk, 45,147 (1978).

35 POLGAR, K. and SKVORTSOV, A.P., Opt. Spekttrosk, 59, 229 (1985) .

36 PRATHER. L.J., National Bureau of Standards Monograph 19. issued february 24, 1961.

37 RAUBER, A., Current Topics in Materials Science, vol. 1 edited by $E$. Kaldis.. pp. 481, 1978.

38 Stevens, K.W.H., Proc. Phys. Soc. London, 65A, 209, (1952).

39 TINKHAN, M., Group Theory and Quantum Mechanics, MacGraw-Hill Book Company, 1964.

40 VIVIEN, D., Rev. Phys. Appl., 21,709 (1986).

41 WEBER, M.J., Phys. Rev. , 156,231 (1967).

42 WEBER, M.J.; BASS, $M$ and deMARS, G.A., J. Appl. Phys., 42,301 (1971).

43 WEBER. M.J.; BASS, M. : VARITIMOS, T.E. and BUA, D.P., IEEE J. Quantum Eletronics, QE-9, 1079 (1973) .

44 WEBER, H.P. and LUTHY, W., Tunable Solid State Lasers II. Springer Series in Optical Sciences, 308 (1986).

45 WEIS, R.S.: GAYLORD, T.K., App 1. PhYs, A37, 191 (1985) 
46 WIGNER, E.P.. Group Theory and its Applications to Quantum Mechanics of Atomic Spectra. Academic Press, New York and London, 1959 .

47 WYBOURNE, B.G., J. Chem Phys., 32,639 (1960).

48 YEN, W.M. and SELZER, P.M., Laser Spectroscopy of Solids, Springer-Verlag. Berlin Heidelberg and New York, 1981. 
APENDICE A

OPERADORES EQUIVALENTES

Consideremos um operador $T$ que atua sobre uma funçăo de onda \# , o resultado sendo uma funça $\varnothing$

$$
\varnothing=T^{*} .
$$

Vamos considerar uma trastormaçăo pertencendo a um grupo $G$ que transforma em e $\varnothing$ em $\varnothing$. De acordo com (A-1) nos obtemos as seguintes relações

$$
\varnothing=\mathrm{RTR}^{-1+\psi}=\mathrm{T}^{\top} \psi
$$

O operador $I=R^{-1}$ é considerado com a transformada de $T$ sob a transformação R.

Uma classe muito importante de operadores são os tensores irredutfveis. Pela definição, um operador tensorial $T_{k}$ de ordem $k$ e um conjunto de $2 k+1$ operadores $T_{k} a$, onde $q=k, k-1, \ldots-k$, - qual sob as rotações do grupo espacial se transforma de acordo com a representaçăo $D^{k}$, isto é,

$$
R T_{k}^{a} R^{-1}=\sum_{a} D^{k} a^{n} a(R) T_{k}^{a}
$$

Se tomarmos $R$ como rotaçoes infinitesimais $R=1+i \in J_{x}$ e $R=1+i \in J_{ \pm}$, onde $J_{ \pm}=J_{x} \pm J_{\nu}$, vemos que de $(A-3)$ obtemos

$$
\left(1-i \in J_{x}\right) T_{k} a\left(1+i \in J_{x}\right)=\sum_{q^{*}}\left(k q^{\prime} i 1-i \in J_{x} i k q\right) T_{k} a^{\prime \prime}
$$

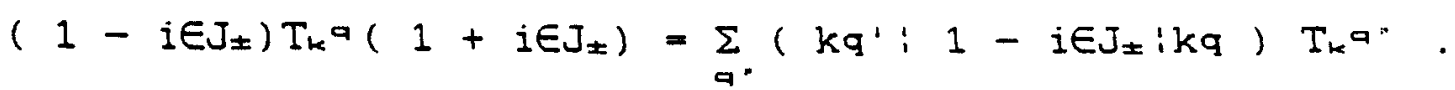


$E^{2}$ e sabendo que

$$
\begin{gathered}
\left\langle j, m_{i} J_{ \pm} i j, m+1\right\rangle=\{j(j+1)-m(m+1)\}^{m} \\
\left\langle j, m i J_{x} i j, m\right\rangle=m
\end{gathered}
$$

obtemos

$$
\begin{gathered}
{\left[J_{z}, T_{k}^{a}\right]=q T_{k:}} \\
{\left[J_{ \pm}, T_{k}^{a}\right]=\{k(k+1)-q(q \pm 1)\} T_{k}^{a \pm 1}}
\end{gathered}
$$

A importancia dos tensores irredutiveis origina-se do seguinte teorema conhecido na literatura como teorema de WignerEckart. Dado um operador tensorial $T_{k}{ }^{a}$, considere o conjunto de todos os elementos de matriz ( $\left.\alpha j m^{\prime} T_{k}{ }^{\prime} \alpha^{\prime} j^{\prime} m^{\prime}\right)$ onde $\alpha e \alpha^{\prime}$ significa qualquer parametro extra que deve ser necessaribmente para especificar os estados $\mid j m>$ e $i j ' m$ '? Para valores fixados de $\alpha, j, k, \alpha^{\prime}, j^{\prime}, k^{\prime}$ existe ao todo $(2 j+1)(2 k+1)\left(2 j^{\prime}+1\right)$ elementos de matriz. O teorema de Wigner-Eckart estabelece que todos estes elementos de matriz são univocamente determinados por um simples fator multiplicativo. Este teorema é usualmente expressado na seguinte forma :

$\left(\alpha j m: T_{k}{ }^{a} ; \alpha^{\prime} j^{\prime} m^{\prime}\right)=\frac{1}{2 J+1}\left(\alpha j i i T_{k} a_{i} i \alpha^{\prime} j^{\prime}\right)\left\langle j^{\prime} m^{\prime}: k q i j j^{\prime} k ; j m\right\rangle \quad(A-7)$ onde $\left(\alpha j: i T_{k} a_{i} \alpha^{\prime} j^{\prime}\right)$ é independente dos numeros quanticos magnéticos $\mathrm{m}^{\prime}, \mathrm{q}, \mathrm{m}$.

Uma forma um pouco diferente de aplicar o teorema de WignerEckart, algumas vêzes mais conveniente do que usar a formula (A-7) , principalmente no cálculo dos elementos da diagonal $j=j^{\prime}$ e $\alpha=\alpha^{\prime}$, é o conhecido metodo dos operadores equivalentes . Considere uma componente $S_{k}{ }^{a}$ de um operador tensorial cujos elementos de matriz sao, por alguma razao. 
particularmente facil de calcular, como por exemplo

$$
S_{2}=3 J_{x}=-J(J+1)
$$

para o qual

$$
\left(\alpha j m: S_{2} \circ: \alpha j m^{\prime}\right)=\delta_{m m} \cdot\left\{3 m^{2}-J(J+1)\right\} \quad(A-9)
$$

Se estamos interessados no operador $T_{2}^{0}=\sum\left(3 z_{0}^{2}-r_{0}^{2}\right)$ iphde onde a soma é sobre todos os elétrons do ion, o teorema de Wigner-Eckart deixa-nos capaz escrever que dentro do estado degenerado $(\propto, j)$

$$
T_{2}{ }^{0}=B S_{2}{ }^{\circ}
$$

onde

$B\left[3 m_{0}{ }^{2}-J(J+1)\right]=\left(\alpha, j, m_{0}: T_{2}^{c}: \alpha, j, m_{0}\right)=\int^{\psi *}(r): \Sigma\left(3 z_{0}{ }^{2}-r_{p}=\right): \psi(r) d r$ 


\section{APENDICE B}

ELEMENTOS DE MATRIZ DOS OPERADORES EQUIVALENTES DE ORDEM $2,4 E 6^{2}$

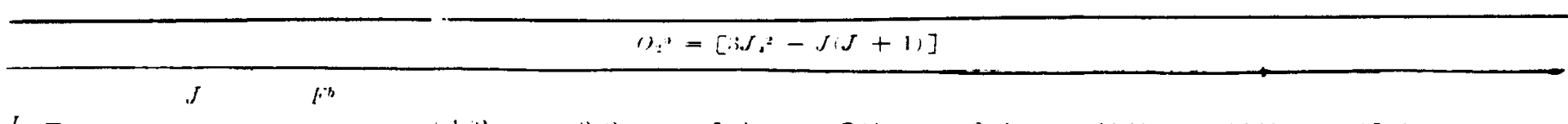

\begin{tabular}{|c|c|c|c|c|c|c|c|c|c|}
\hline$I .=$ & & $\pm 1: 2$ & $\pm 3:$ & \pm 2 & $\pm \pi$ & $\pm 9 / 2$ & $\pm 11,:$ & $\pm 1: 12$ & \pm 15 \\
\hline $1 \div 2$ & () & 10 & $\cdots$ & - & - & -- & - & - & - \\
\hline$\because \because$ & $: 3$ & -1 & 1 & & $\cdots$ & $\cdots$ & -- & - & $\cdots$ \\
\hline$\therefore 2$ & $\because$ & -1 & -1 & $\therefore$ & & .. & -- & $\cdots$ & .. \\
\hline$\overline{-}$ & $\because$ & $\cdots i$ & $\therefore$ & 1 & $i$ & - & - & - & - \\
\hline $9:$ & $1 i$ & -4 & $-i$ & -1 & 2 & ii & - & - & - \\
\hline 112 & 1 & $-i i$ & $\cdots$ & $-1 i$ & 1 & $\therefore i$ & $\pi i$ & - & - \\
\hline $1: 12$ & ti & ' & $i$ & $\therefore$ & $\because$ & $\because$ & $i$ & $1 .:$ & - \\
\hline $1: 1$ & 3 & 21 & $\cdot 1 !$ & $1 . i$ & $!$ & -1 & !) & $\because 1$ & i.i \\
\hline
\end{tabular}

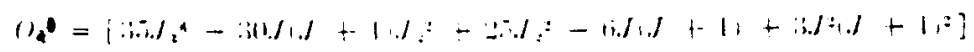

\begin{tabular}{|c|c|c|c|c|c|c|c|c|c|c|}
\hline & $J$ & $F$ & & & & & & & & \\
\hline \multirow{9}{*}{$J_{.}=$} & & & \pm 12 & $\pm: \because$ & $\pm i / 2$ & $\pm i ;$ & $\pm 9: 2$ & $\pm 11 / 2$ & $\pm 1: 3 / 2$ & $\pm 1 ;$ \\
\hline & 12 & 0 & 11 & $\cdots$ & -. & $\ldots$ & -- & - & - & - \\
\hline & $\therefore, \because$ & 11 & 11 & 11 & - & & - - & $\cdots$ & - & 一 \\
\hline & $\therefore 2$ & (in) & $\because$ & $::$ & 1 & & & $\cdots$ & $\cdots$ & - \\
\hline & $7 \because$ & 1,11 & ! & -3 & $-1 ; 3$ & $i$ & - - & - & - & -- \\
\hline & 92 & $\therefore .4$ & 11 & 3 & -17 & -2 & 15 & - & - & - \\
\hline & $11: 2$ & $1: 11$ & Sי & $1:$ & $-1: 3$ & $-: 33$ & $-2 \pi$ & 33 & - & - \\
\hline & $1: 12$ & in & 111 & iii & $1: i$ & 93 & $-1: 32$ & $-i T$ & 113 & 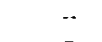 \\
\hline & $1 \therefore 2$ & 11 & $1 ! 1$ & $13 ! 9$ & $\therefore:$ & 1111 & $\ldots-111$ & $\because \cdots ! 1$ & $! 1$ & $\because \div$ \\
\hline
\end{tabular}

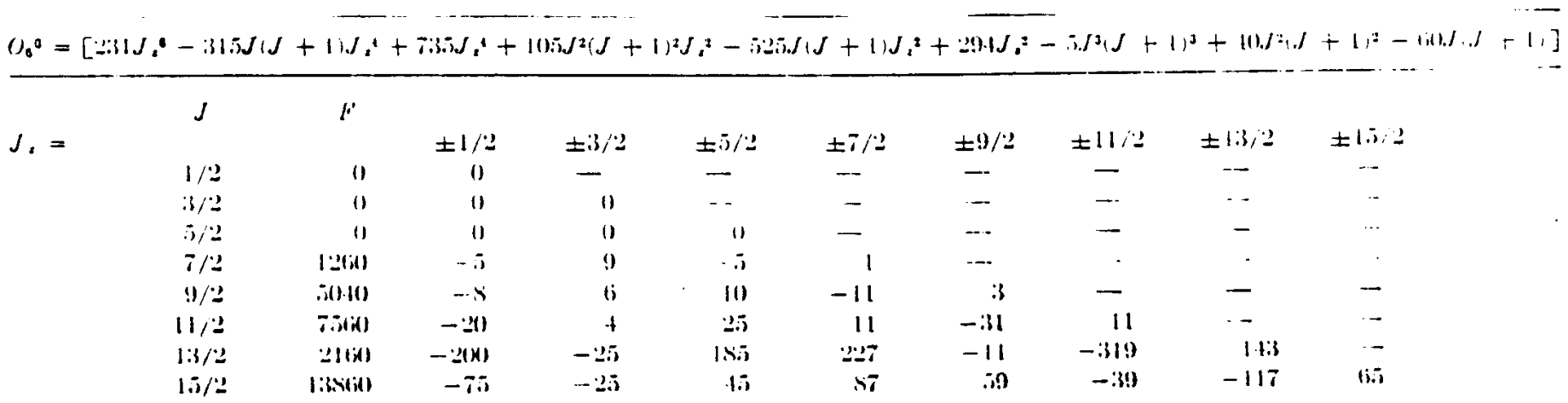




\begin{tabular}{|c|c|c|c|c|c|c|}
\hline$(8: 1 \cdot 88) \cdot$ & Ge: ' & $2:-c^{2}$ & ts & $\subseteq$ 'it. & I $2(n)$ & $\therefore ! !$ \\
\hline- & $c x+1$ & $88^{\wedge} !$ & $99 !$ & (1) & $0=1$ & $\because: 1$ \\
\hline - & - & rot 1 & $m: 12$ & $\because A_{i}$ & (m): & $\therefore 11$ \\
\hline - & - & - & $16 \cdot$ & +1 & (x): & $\approx \pi$ \\
\hline - & - & - & - & 21 & 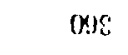 & $\pi:$ \\
\hline 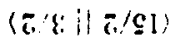 & $(\sigma: 1 \| \sigma / c \mid)$ & $\left(\sigma^{\prime} / 1-\|\sigma /\|\right)$ & $\left\langle\sigma^{\prime}(q-\| \sigma)\right.$ & $(8)-1\rangle=2$ & .4 & $r$ \\
\hline
\end{tabular}

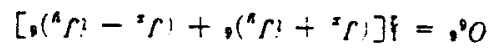

\begin{tabular}{|c|c|c|c|c|c|c|c|}
\hline ד1 & $16 i^{\circ} \varepsilon$ & 0 & $11^{1}:-$ & 19:- & บั11: & (Kitil & 81 \\
\hline$-\cdot$ & $9 \mathrm{~S}^{-} \wedge \mathrm{9}$ & 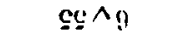 & $99^{1}+-$ & (1) $(i \pi-$ & $\left(1^{1}+1-\right.$ & mer: & -81 \\
\hline$-\cdot$ & - & $s \mathcal{E}^{\wedge} \delta$ & $a^{\wedge} 2$ & i. ${ }^{1} 91-$ & $+1 \wedge 2 \pi-$ & $\mathrm{c} \wedge \cap \&$ & $8: 11$ \\
\hline - & - & - & $1 \pi \%$ & $+11-$ & 1 $1^{\wedge}-$ & 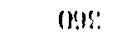 & $2 / 6$ \\
\hline$\cdots$ & - & - & - & $\sin \bar{\pi}$ & ga:- & $m:$ & $\sigma^{\prime} 2$ \\
\hline -. & - & - & - & - & 0 & 0 & $\pi$ \\
\hline (8) & $(\pi)+\pi / 81)$ & $\langle\pi / \subseteq\|\pi /\|\rangle$ & $\langle\pi / 8 \| \pi / 6)$ & $\left\langle\bar{c} \mid \boldsymbol{1} \| \sigma^{\prime}: 2\right\rangle$ & $\langle-1-\| r \mid d\rangle$ & $d$ & $r$ \\
\hline
\end{tabular}

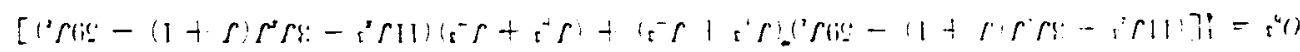

\begin{tabular}{|c|c|c|c|c|c|c|c|}
\hline cst' 1 & $10 \wedge 06$ & sit $\wedge_{t}$ & $\|^{\wedge} 0:$ & $18-10$ & $\cot { }^{\prime} t$ & 4 & $\therefore \because$ \\
\hline- & $9 \times 0 \div$ & $5: \wedge !$ & $x y \wedge$ & $9 x_{01}$ & 91911 & $\varepsilon$ & 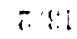 \\
\hline -. & - & $\varepsilon x$ & $g^{\wedge} \varepsilon$ & i. 1 & +11 & $91-1$ & $\therefore 11$ \\
\hline - & - & - & $12 \times 9$ & +118 & 110 & \& & $i \dot{i}$ \\
\hline- & - & - & - & $\cos$ & $\mathrm{c} \wedge \mathrm{b}$ & $\&$ & $\div \div$ \\
\hline- & - & - & - & - & 01 & $\varepsilon$ & $\therefore$ \\
\hline$\therefore 6 \| \approx 91$ & $\langle z / 2 \| z / \varepsilon 1\rangle$ & $\langle\bar{\sigma} / \subseteq\|\bar{\sigma} /\|\rangle$ & $\left\langle\sigma^{\prime} \varepsilon \sigma^{j}\right.$ & $\langle\sigma|1| \bar{\sigma}: 2$ & $\langle\pi|-| \pi / s$ & $A$ & $\Gamma$ \\
\hline
\end{tabular}

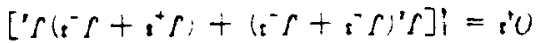


APENDICE C

TABELAS UTILIZADAS NA TEORIA DE GRUPOS ${ }^{29}$

TABELA C1 - TABELA DE CARACTERES E FUNÇరES DE BASE PARA O GRUPO O

\begin{tabular}{|c|c|c|c|c|c|c|c|c|c|c|}
\hline 0 & E & $\bar{E} 8$ & $C_{3} 8$ & & $\begin{array}{l}3 \mathrm{C}_{2} \\
3 \overline{\mathrm{C}}_{2} 6 \mathrm{C}_{4}\end{array}$ & $6 \overline{\mathrm{C}}$ & $\begin{array}{l}6 \mathrm{C}_{2} \\
6 \overline{\mathrm{C}}_{2}\end{array}$ & & & \\
\hline$T_{1}$ & $E$ & $\bar{E} .8$ & $\mathrm{C}_{3}$ & $8 \overline{\mathrm{C}}_{3}$ & $\begin{array}{l}3 \mathrm{C}_{2} \\
3 \overline{\mathrm{C}}_{2}\end{array}$ & $6 \bar{S}$ & $\begin{array}{l}60_{d} \\
6 \bar{o}_{d} \\
\end{array}$ & $\begin{array}{l}\text { Time } \\
\text { Lnv. }\end{array}$ & $\begin{array}{l}\text { Bases } \\
\text { for } 0\end{array}$ & $\begin{array}{l}\text { Bases } \\
\text { for } T_{0}\end{array}$ \\
\hline$\Gamma_{1}$ & 1 & 1 & 1 & 1 & 11 & 1 & 1 & $a$ & $R$ & $R$ or $x y z$ \\
\hline$\Gamma_{2}$ & 1 & 1 & 1 & 1 & $1-1$ & -1 & -1 & $a$ & $x y z$ & $S_{z} S_{3} S_{z}$ \\
\hline$r_{3}$ & 2 & 2 & -1 & -1 & 20 & 0 & 0 & a & $\begin{array}{c}\left(2 z^{2}-x^{2}-y^{2}\right) \\
\sqrt{3}\left(x^{2}-y^{2}\right)\end{array}$ & $\begin{array}{c}\left(2 z^{2}-x^{2}-y^{2}\right) \\
\sqrt{3}\left(x^{2}-y\right.\end{array}$ \\
\hline$r \cdot$ & 3 & 3 & 0 & 0 & $\begin{array}{ll}-1 & 1\end{array}$ & 1 & -1 & a & $\dot{S}_{z}, \dot{s}_{1}, \dot{\Xi}_{2}$ & $S_{z}, S_{y}, S_{z}$ \\
\hline$\Gamma_{\mathrm{s}}$ & 3 & 3 & 0 & 0 & $-1-1$ & -1 & 1 & a & $\because z, x z, x y$ & $. x, y, z$ \\
\hline .1 & 2. & & 1 & -1 & $0 \sqrt{2}$. & $-\sqrt{2}$ & 0 & $c$ & $\begin{array}{l}\text { of } 2,-12), \\
\text { of } 2,1,2)\end{array}$ & 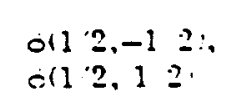 \\
\hline$r$ & 2 & $\therefore$ & 3 & -1 & $0-12$ & $\therefore \bar{I}$ & 0 & $=$ & $r_{:} \cdot r_{:}$ & $\Gamma_{0} \cdot \Gamma_{z}$ \\
\hline \multirow[t]{2}{*}{$r_{1}$} & 4 & $-i$ & -1 & 1 & $0 \quad 0$ & 0 & $n$ & $\therefore$ & 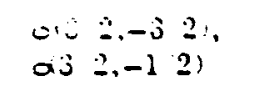 & 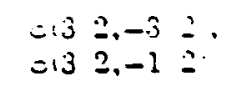 \\
\hline & & & & & & & & & $\begin{array}{l}0(3,2,1 / 2), \\
\phi(3 / 2,3 / 2)\end{array}$ & $\begin{array}{l}\text { o(3,2,1:2), } \\
\phi(3,2,3,2)\end{array}$ \\
\hline
\end{tabular}

TABELA C2 - TABELA DE MULTIPLICAÇO PARA O GRUPO O

\begin{tabular}{|c|c|c|c|c|c|c|c|}
\hline$r_{1} r_{2}$ & $r_{3}$ & $\Gamma_{4}$ & $r_{3}$ & $\Gamma_{6}$ & $r$ & $r$ & \\
\hline \multirow{8}{*}{$\begin{array}{l}r_{1} r_{2} \\
r_{1}\end{array}$} & $\Gamma_{3}$ & $\Gamma_{4}$ & $\Gamma_{s}$ & $r_{0}$ & $\Gamma$ & $\Gamma_{1}$ & $r_{2}$ \\
\hline & $r_{3}$ & $r_{s}$ & $\Gamma_{4}$ & $\Gamma$ & $r_{0}$ & $\Gamma_{2}$ & $\Gamma_{2}$ \\
\hline & $r_{1}+r_{2}+r_{3}$ & $r_{4}+\Gamma_{3}$ & $r_{4}+r_{3}$ & 1. & $r$ & $\Gamma_{6}+\Gamma_{7}+\Gamma_{2}$ & $\Gamma$ \\
\hline & & $\Gamma_{1}+\Gamma_{3}+\Gamma_{4}+\Gamma_{3}$ & $r_{2}+r_{3}+r_{4}+r_{3}$ & $\Gamma_{6}+\Gamma_{0}$ & $\Gamma_{2}+\Gamma_{0}$ & $\Gamma_{\mathrm{b}}+\Gamma_{7}+2 \Gamma_{\mathrm{s}}$ & $\Gamma$ \\
\hline & & & $r_{1}+r_{3}+r_{1}+r_{3}$ & $1 ;+1$ & $1: 4 r$ & $1 \because+r+2 r_{0}$ & $1 ;$ \\
\hline & & & & $r_{1}+r_{4}$ & $r_{2}+r_{5}$ & $\Gamma_{3}+\Gamma_{4}+\Gamma_{3}$ & $\Gamma$ \\
\hline & & & & & $r_{1}+r_{4}$ & $r_{3}+r_{4}+r_{3}$ & $r$, \\
\hline & ${ }^{\circ}$ & & & & & $r_{1}+r_{2}+r_{3}$ & 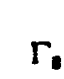 \\
\hline
\end{tabular}


TABELA C3 - TABELA DE COMPATIBILIDADE COM O GRUPO DAS ROTAÇOES PARA O GRUPO 0 .

\begin{tabular}{|c|c|}
\hline$D_{6}^{2}$ & $\Gamma_{1}$ \\
\hline$D_{1}^{2}$ & $\Gamma_{4}$ \\
\hline$D_{2}^{2}$ & $\Gamma_{1}+\Gamma_{1}$ \\
\hline$D_{3}^{2}$ & $\Gamma_{2}+\Gamma_{1}+\Gamma_{3}$ \\
\hline$D_{4}^{s}$ & $\Gamma_{1}+\Gamma_{1}+\Gamma_{1}+\Gamma_{3}$ \\
\hline$D_{3}^{2}$ & $\Gamma_{3}+2 \Gamma_{4}+\Gamma_{3}$ \\
\hline$D_{0}^{z}$ & $r_{1}+r_{2}+\Gamma_{1}+r_{1}+2 r_{3}$ \\
\hline$D_{1 / 2}^{2}$ & $\mathrm{I}_{6}$ \\
\hline$D_{3 / 2}^{2}$ & $r_{1}$ \\
\hline$D_{s / 2}^{2}$ & $r,+r$ \\
\hline$D_{i / 2}^{*}$ & $10+1,+10$ \\
\hline$n_{n / 2}^{2}$ & 1.21 \\
\hline$D_{11 / 2}^{2}$ & $r_{6}+1 ;+21$ \\
\hline $\begin{array}{l}D_{1 / 2}^{0} \\
D_{r / 2}\end{array}$ & $\begin{array}{l}r_{6}+2 r_{1}+2 r_{1} \\
r_{6}+r_{7}+3 r_{8}\end{array}$ \\
\hline
\end{tabular}

TABELA C4-TABELA DE CARACTERES E FUNÇOES DE BASE PARA O GRUPO On.

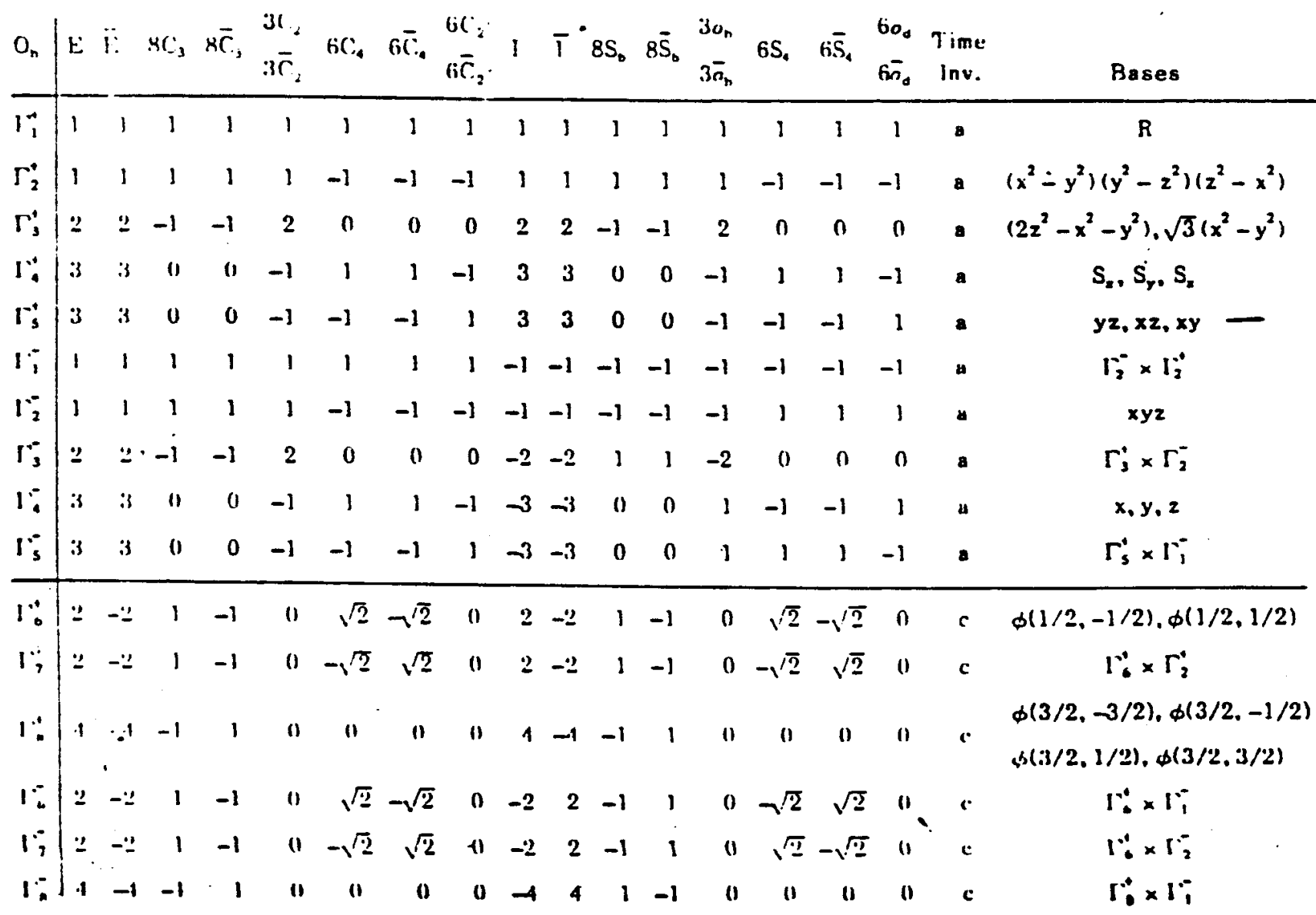


TABELA C5 - TABELA DE COMPATIBILIDADE.

\begin{tabular}{|c|c|c|c|c|c|c|c|c|}
\hline $\mathrm{O}_{3}$ & $\Gamma_{1}^{*} \Gamma_{2}^{*}$ & $\Gamma_{3}^{*}$ & $\Gamma:$ & $r_{s}$ & $\Gamma_{1}^{*} \Gamma_{2}^{-}$ & $\Gamma_{3}^{-}$ & $\Gamma_{i}^{-}$ & $\Gamma_{s}^{-}$ \\
\hline 0 & $\Gamma_{1} \Gamma_{2}$ & $\Gamma_{3}$ & $\Gamma$ & $\Gamma_{s}$ & $\Gamma_{1} \Gamma_{2}$ & $\Gamma_{3}$ & $r$ & $\Gamma_{s}$ \\
\hline$T_{0}$ & $r_{1} r_{2}$ & $\Gamma_{3}$ & $\Gamma$. & $\Gamma_{s}$ & $\Gamma_{:} \Gamma_{:}$ & $\Gamma_{3}$ & $\Gamma_{s}$ & $\Gamma$ \\
\hline$T_{n}$ & $\Gamma_{i}^{*} \Gamma_{1}^{*}$ & $\Gamma_{2}^{+}+\Gamma_{3}^{+}$ & $\Gamma^{*}$ & $\Gamma$ & $\Gamma_{i}^{*} r_{i}^{\circ}$ & $\Gamma_{2}^{-}+\Gamma_{2}^{-}$ & $\Gamma_{4}^{-}$ & $\Gamma^{-}$ \\
\hline $\mathrm{D}_{\mathrm{Ar}}$ & $\Gamma_{1}^{*} \Gamma_{3}^{\prime}$ & $\Gamma_{1}^{*}+\Gamma_{3}^{+}$ & $\Gamma_{i}^{\prime}+\Gamma_{i}^{*}$ & $r_{i}^{*}+r_{i}^{*}$ & $r_{1}^{*} r_{i}$ & $\Gamma_{1}^{-}+\Gamma_{3}^{-}$ & $r_{i}^{-}+\Gamma_{s}^{*}$ & $\Gamma_{+}^{-}-\Gamma_{i}^{-}$ \\
\hline$D_{3 d}$ & $r_{:}^{*} r_{2}^{*}$ & $\Gamma_{3}^{*}$ & $\Gamma_{2}^{*}+\Gamma_{2}^{*}$ & $\Gamma_{:}^{*}+\Gamma_{3}^{+}$ & $r_{:}^{-} r_{2}^{-}$ & $\Gamma_{3}^{-}$ & $\Gamma_{2}^{*}+\Gamma_{3}^{*}$ & $\Gamma_{i}^{-}+\Gamma_{3}^{-}$ \\
\hline$C_{1 n}: H(z)$ & $\Gamma_{1}^{*} \Gamma_{2}^{*}$ & $r_{i}^{*}+r_{z}^{*}$ & $\Gamma_{1}^{+}+\Gamma_{2}^{+}+\Gamma$ & $\Gamma_{:}^{*}-\Gamma_{3}^{*}+\Gamma_{4}^{*}$ & $\Gamma_{i}^{*} r_{i}^{-}$ & $\Gamma_{1}^{-}+\Gamma_{i}^{-}$ & $\Gamma_{1}^{-}-\Gamma_{3}^{-}+\Gamma$ & $\Gamma_{2}^{-}-\Gamma_{2}^{-}+\Gamma_{1}^{-}$ \\
\hline$C_{2 n}: H(n)$ & $\Gamma_{i}^{*} \Gamma^{*}$ & $\Gamma_{1}^{*}+\Gamma_{2}^{\prime}$ & $2 \Gamma_{i}^{*}+\Gamma_{i}^{\prime}$ & $2 \Gamma_{1}+\Gamma_{2}^{*}$ & $\Gamma_{:}^{-} \Gamma_{0}^{-}$ & $r_{i}^{-}+r_{i}^{-}$ & $2 \Gamma_{i}^{-}+\Gamma_{i}^{-}$ & $2 \Gamma_{:}^{-}-\Gamma_{z}^{-}$ \\
\hline$C_{31}: H(x)$ & $\Gamma_{i}^{*} \Gamma_{i}^{*}$ & $\Gamma_{2}^{*}+\Gamma_{3}^{*}$ & $\Gamma_{1}^{\prime}+\Gamma_{2}^{\prime}+\Gamma^{\prime}$ & $\Gamma^{*}-\Gamma_{2}^{+}+\Gamma_{3}^{+}$ & $r_{i} r_{i}$ & $\Gamma_{z}^{-}+\Gamma_{3}^{-}$ & $\Gamma_{i}^{-}+\Gamma_{2}^{-}+\Gamma_{3}^{-}$ & $\Gamma_{1}^{-}+\Gamma_{2}^{*}+\Gamma_{2}^{-}$ \\
\hline$C_{4 v}: E(z)$ & $r_{1} \Gamma_{3}$ & $\Gamma_{1}+\Gamma_{3}$ & $\Gamma_{2}+\Gamma_{s}$ & $\Gamma_{t}+\Gamma_{s}$ & $\Gamma_{2} \Gamma_{4}$ & $\Gamma_{2}+\Gamma_{4}$ & $\Gamma_{1}+\Gamma_{s}$ & $\Gamma_{3}+\Gamma_{5}$ \\
\hline$C_{2 v}: E(v)$ & $\Gamma_{1} \Gamma_{2}$ & $\Gamma_{1}+\Gamma_{2}$ & $\Gamma_{0}+\Gamma_{0}+1$ & $=-\Gamma_{3}+\Gamma_{4}$ & $\Gamma_{3} \Gamma$ & $\Gamma_{3}+$ & $\Gamma_{1}+\Gamma_{2}-$ & $\Gamma_{1}+\Gamma_{2}+\Gamma_{3}$ \\
\hline$C_{3 r}: E(u)$ & $\mid r_{1} r_{2}$ & $\Gamma_{3}$ & $\Gamma_{2}+\Gamma_{3}$ & $\Gamma_{1}+\Gamma_{3}$ & $\Gamma_{2} \Gamma_{i}$ & $r_{3}$ & $\Gamma_{1}+\Gamma_{3}$ & $\Gamma_{2}+\Gamma_{z}$ \\
\hline
\end{tabular}

\begin{tabular}{|c|c|c|c|c|c|c|}
\hline 0. & $\Gamma_{0}^{*}$ & $r$ & $r$ & $\Gamma_{\epsilon}^{-}$ & $r:$ & $\Gamma_{8}^{-}$ \\
\hline 0 & $\Gamma_{\mathrm{t}}$ & $\Gamma_{i}$ & $\Gamma_{i}$ & $\Gamma_{0}$. & $\Gamma$ & $\Gamma_{3}$ \\
\hline$T_{c}$ & $\Gamma_{0}$ & $\Gamma_{i}$ & $r$ & $\Gamma$ & $r_{e}$ & $\Gamma_{\bullet}$ \\
\hline$T_{*}$ & $\Gamma$ & $\Gamma_{:}^{*}$ & $r:-r:$ & $\Gamma_{\vdots}^{-}$ & $r_{i}^{*}$ & $r_{0}^{-}-r^{-}$ \\
\hline$D_{s:}$ & $\Gamma^{*}$ & $r:$ & $r_{i}^{*}-r_{i}$ & $r_{c}^{-}$ & $\Gamma:$ & $r_{i}^{-}+r^{-}$ \\
\hline$D_{1:}$ & $r:$ & $r$ & $\Gamma_{i}^{*}-r_{i}^{-}-\Gamma_{0}^{*}$ & $r$ & $r_{s}^{-}$ & $\Gamma_{+}^{-}-\Gamma_{i}^{-}+\Gamma_{i}^{-}$ \\
\hline$C_{48}: H(z)$ & $r_{3}^{*}+r_{6}^{*}$ & $\Gamma_{i}^{*}+\Gamma_{i}^{\prime}$ & $\Gamma_{i}^{\prime}-\Gamma_{i}^{-}-\Gamma_{i}^{*}+\Gamma_{i}^{*}$ & $\Gamma_{s}^{-}-\Gamma_{0}^{-}$ & $r+r$ & $r_{5}^{-}-r_{6}^{-}+r_{7}^{-}-r_{3}^{-}$ \\
\hline$C_{22}: H(v)$ & $\Gamma_{3}^{*}+\Gamma_{4}^{*}$ & $\Gamma_{3}^{*}+\Gamma_{i}^{*}$ & $2 \Gamma_{j}^{-}+2 \Gamma_{4}^{+}$ & $\Gamma_{3}^{-}+\Gamma_{4}^{-}$ & $\Gamma_{3}^{-}+\Gamma_{4}^{-}$ & $2 \Gamma_{3}^{-}+2 \Gamma_{4}^{-}$ \\
\hline$C_{3 t}: H(w)$ & $\Gamma_{4}^{*}+\Gamma_{5}^{*}$ & $\Gamma_{4}^{*}+\Gamma_{s}^{*}$ & $\Gamma_{4}^{\prime}-\Gamma_{3}^{*}+2 \Gamma_{6}^{+}$ & $\Gamma_{4}^{-}+\Gamma_{s}^{-}$ & $\Gamma_{4}^{-}+\Gamma_{3}^{-}$ & $\Gamma_{1}^{-}+\Gamma_{s}^{-}+2 \Gamma_{b}^{-}$ \\
\hline$C_{40}: E(z)$ & $\Gamma_{6}$ & $\Gamma_{1}$ & $\Gamma_{6}+\Gamma$ & $\Gamma_{6}$ & $\Gamma$ & $\Gamma_{0}+\Gamma_{1}$ \\
\hline$C_{2 v}: E(v)$ & $\Gamma_{s}$ & $\Gamma_{3}$ & $2 \Gamma_{s}$ & $\Gamma_{s}$ & $\Gamma_{s}$ & $2 \Gamma_{3}$ \\
\hline$C_{2 v}: E(w)$ & $\Gamma_{4}$ & $\Gamma$ & $\Gamma_{1}-\Gamma_{3}+\Gamma_{1}$ & r. & $\Gamma$ & $\Gamma_{4}+\Gamma_{3}+\Gamma_{0}$ \\
\hline
\end{tabular}


TABELA C6 - TABELA DE CARACTERES E FUNÇOES DE BASE PARA OS GRUPOS $D=E C \Xi v$.

\begin{tabular}{|c|c|c|c|c|c|c|c|c|c|}
\hline$D_{1}$ & $E$ & $\bar{E}$ & $2 C_{3}$ & $2 \bar{C}_{1}$ & $3 C_{i}$ & $3 \vec{C}_{:}^{:}$ & & & \\
\hline$C_{3 v}$ & $E$ & $\bar{E}$ & $2 C_{3}$ & $2 \overline{\mathrm{C}}_{3}$ & 30. & $\hat{\jmath} \overrightarrow{.}$ & Ins. & for $D_{3}$ & for $C_{3}$ \\
\hline$\Gamma_{1}$ & 1 & 1 & 1 & 1 & 1 & 1 & a & $R$ & $R$ or $z$ \\
\hline$r:$ & 1 & 1 & 1 & 1 & -1 & -1 & $a$ & $S_{z}$ or 2 & $S_{z}$ \\
\hline$\Gamma_{3}$ & 2 & 2 & -1 & -1 & 0 & 0 & a & $\begin{array}{l}\left(S_{y}-i S_{y}\right) \\
-\left(S_{y}+i S_{y}\right)\end{array}$ & $\begin{array}{l}\left(\Sigma_{z}-i S_{y}\right) \\
-\left(\Sigma_{z}-i S_{y}\right)\end{array}$ \\
\hline$\Gamma$ & 2 & -2 & 1 & -1 & 0 & 0 & c & $\begin{array}{c}\phi(1 / 2,-12) \\
\phi(1 / 2,1,2)\end{array}$ & $\begin{array}{l}\phi(1 ' 2,-1 / 2) \\
0(1 ' 2,1 / 2)\end{array}$ \\
\hline$r_{s}$ & 1 & -1 & $-i$ & 1 & i & -1 & b & $\begin{array}{c}\phi(3 / 2,-3,2) \\
-i \phi(3 / 2.3: 2)\end{array}$ & 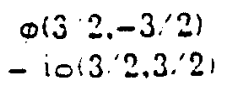 \\
\hline$r=$ & 1 & -1 & -1 & 1 & -1 & 1 & b & $\begin{array}{l}-i o(3: 2.3 / 2) \\
-i \phi(3 / 2 .-3 / 2)\end{array}$ & $\begin{array}{l}-1013 \cdot 2.3 \cdot 2) \\
1-1013,2 .-3,2\end{array}$ \\
\hline
\end{tabular}

TABELA C7 - TABELA DE MULTIPLICAÇAO PARA OS GRUPOS D $=$ E C $\approx u$

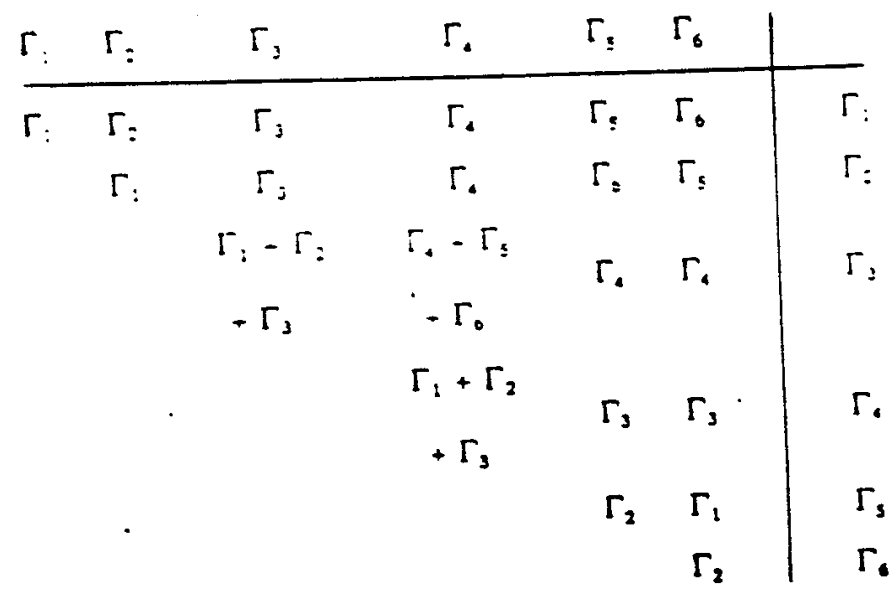

TABELA C8 - TABELA DE COMPATIBILIDADE COM O GRUPO DAS ROTAÇOES PARA O GRUPO D $=$.

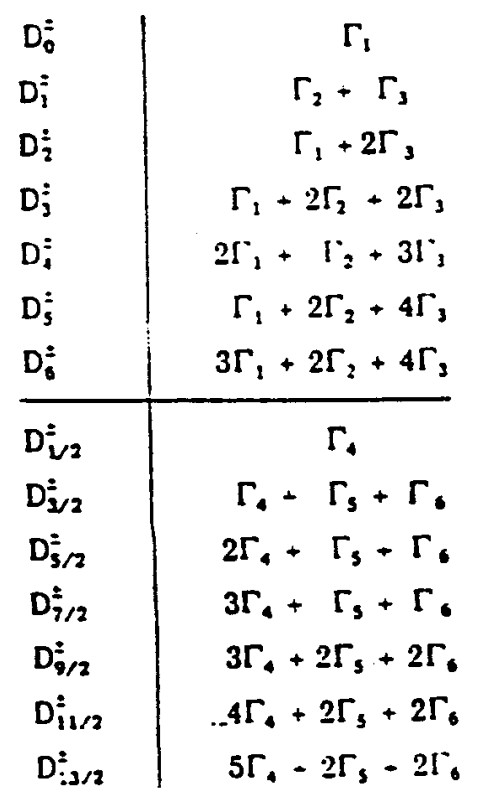


TABELA C9 - TABELA DE COMPATIBILIDADE COM O GRUPO DAS ROTAÇOES PARA O GRUPO Csu .

\begin{tabular}{|c|c|c|c|}
\hline$L_{i}^{*}$ & $1:$ & $b_{c}^{i}$ & $r_{2}$ \\
\hline D: & $r_{2}=r_{2}$ & $\mathrm{D}^{\circ}$ & $r_{1} \cdot r_{2}$ \\
\hline D: & $r_{1}-2 r_{2}$ & L: & $\Gamma_{2}: 2 \Gamma_{3}$ \\
\hline$n_{i}$ & $1:-21=-21=$ & $D_{i}^{-}$ & $2 r_{1}+r_{2}+2 r_{3}$ \\
\hline$L_{i}$ & $21:-i=31=$ & $\because:$ & $\Gamma_{1}+2 \Gamma_{2}+3 \Gamma$ \\
\hline$E_{i}^{*}$ & $r_{2}-2 i_{2}=+r_{3}$ & $U_{s}^{-}$ & $2 \Gamma_{1}+\Gamma_{2}+\because$. \\
\hline$E_{i}^{*}$ & $3 \Gamma_{1}+2 \Gamma_{z}+4 \Gamma_{3}$ & $D_{t}^{-}$ & $2 \Gamma_{1} \cdot 3 i=+3 i=$ \\
\hline$D_{1 / 2}^{*}$ & $r_{4}$ & $D_{1 / 2}^{-}$ & $r$ \\
\hline$D_{3:=}^{*}$ & $\Gamma_{4}+\Gamma_{3}+\Gamma_{0}$ & $D_{3 / 2}^{-}$ & $1+19-1$ \\
\hline$D_{3 / 2}^{+}$ & $2 \Gamma_{4}+\Gamma_{5}+\Gamma_{6}$ & $\mathrm{D}_{S / 2}^{-}$ & $2 ! r_{a}+\Gamma_{0}$ \\
\hline$D_{i / 2}^{0}$ & $3 r_{4}+r_{5}-r_{6}$ & $\mathrm{D}_{i / 2}^{-}$ & $3 r_{4}-\Gamma_{t}-r_{\varepsilon}$ \\
\hline$\Sigma_{9 / 2}^{*}$ & $3 \Gamma_{4}+2 \Gamma_{3}+2 \Gamma_{6}$ & $D_{9,2}^{-}$ & $3 \Gamma_{4}+2 \Gamma_{\leq}-2 ! 。$ \\
\hline$D_{11 / 2}^{*}$ & $4 \Gamma_{4}+2 \Gamma_{\mathrm{s}}+2 \Gamma_{t}$ & $D_{1 / 2}^{-}$ & $41 \mathrm{i}+2 \Gamma_{\mathrm{s}}+2 \mathrm{i}_{\mathrm{t}}$ \\
\hline & $5 \Gamma_{1}+2 \Gamma_{t}+2 \Gamma_{t}$ & $\mathrm{D}_{13,2}^{-}$ & $5 r_{4}+2 ! ?-2 I_{4}$ \\
\hline
\end{tabular}

TABELA C10 - TABELA DE CARACTERES E FUNÇðES DE BASE PARA O Dasa.

\begin{tabular}{|c|c|c|c|c|c|c|c|c|c|c|c|c|c|c|}
\hline$[30$ & $\mathbf{E}$ & $\overline{\mathbf{E}}$ & $2 C_{3}$ & $2 \bar{C}_{3}$ & $3 C_{i}^{\prime}$ & $3 \bar{C}_{2}^{*}$ & 1 & 12 & $2 S$ & $2 \bar{S}_{6}:$ & 30. & $3 \overline{0}$ & $\begin{array}{l}\text { Time } \\
\text { Inv. }\end{array}$ & Bases \\
\hline$\Gamma$ & 1 & 1 & 1 & 1 & 1 & 1 & 1 & 1 & 1 & 1 & 1 & 1 & a & $\mathbf{R}$ \\
\hline$\Gamma_{2}^{*}$ & 1 & 1 & 1 & 1 & -1 & -1 & 1 & 1 & 1 & 1 & -1 & -1 & - & S, \\
\hline$\Gamma_{;}^{+}$ & 2 & 2 & -1 & -1 & 0 & 0 & 2 & 2 & -1 & -1 & 0 & 0 & . & $\begin{array}{r}\left(S_{z}-i S_{y}\right), \\
-\left(S_{z}+i S_{z}\right)\end{array}$ \\
\hline$\Gamma_{1}^{-}$ & 1 & 1 & 1 & 1 & 1 & 1 & -1 & -1 & -1 & -1 & -1 & -1 & a & $2 S_{3}$ \\
\hline$r_{2}^{-}$ & 1 & 1 & 1 & 1 & -1 & -1 & -1 & -1 & -1 & -1 & 1 & 1 & . & $x$ \\
\hline$\Gamma_{2}^{-}$ & 2 & 2 & -1 & -1 & 0 & 0 & -2 & -2 & 1 & 1 & 0 & 0 & a & $\begin{array}{r}(x-i y) \\
-(x+i y) \\
\end{array}$ \\
\hline$r_{\bullet}^{*}$ & 2 & -2 & 1 & -1 & 0 & 0 & 2 & -2 & 1 & -1 & 0 & 0 & c & $\begin{array}{l}\phi(1 / 2,-1 / 2) \\
\phi(1 / 2,1 / 2)\end{array}$ \\
\hline$r_{s}^{*}$ & 1 & -1 & -1 & 1 & i & $-i$ & 1 & -1 & -1 & 1 & i & $-i$ & b & $\begin{array}{r}\phi(3 / 2,-3 / 2) \\
-i \phi(3 / 2,3 / 2)\end{array}$ \\
\hline$\Gamma^{+}$ & 1 & -1 & -1 & 1 & $-i$ & $\mathbf{i}$ & 1 & -1 & -1 & 1 & $-i$ & i & b - & $\begin{array}{l}-(\phi(3 / 2,3 / 2) \\
-i \phi(3 / 2,-3 / 2)\end{array}$ \\
\hline$\Gamma_{4}^{*}$ & 2 & -2 & 1 & -1 & 0 & 0 & -2 & 2 & -1 & 1 & 0 & 0 & c & $\Gamma_{4}^{+} \times \Gamma_{1}^{-}$ \\
\hline$\Gamma_{s}^{-}$ & 1 & -1 & -1 & 1 & i & $-i$ & -1 & 1 & 1 & -1 & $-i$ & i & b & $\Gamma_{i}^{+} \times \Gamma_{1}^{-}$ \\
\hline$\Gamma^{-}$ & 1 & -1 & -1 & 1 & $-\mathbf{i}$ & i & -1 & 1 & 1 & -1 & i & $-i$ & b & $\Gamma_{0}^{+} \times \Gamma_{1}^{-}$ \\
\hline
\end{tabular}


TABELA C11 - TABELA DE CARACTERS E FUNÇOES DE BASE PARA O GRUPO $\mathrm{C} \equiv$.

\begin{tabular}{|c|c|c|c|c|c|c|c|c|}
\hline$C_{3}$ & $E$ & $\bar{E}$ & $C_{2}$ & $\bar{c}_{z}$ & $\sigma_{!}:$ & $\bar{C}_{3}^{-i}$ & $\begin{array}{l}\text { Time } \\
\text { Inv. }\end{array}$ & Bases \\
\hline$r:$ & 1 & 1 & 1 & 1 & 1 & 1 & a & 2 or 5 \\
\hline$\Gamma:$ & 1 & 1 & $\overrightarrow{r r}^{2}$ & $\omega:$ & سـ & - & $b$ & $-i i x-i y$ ) or $-i S_{z}-i \Sigma_{y}$ \\
\hline$\Gamma_{3}$ & $\vdots$ & 1 & - & $\because$ & ش: & $\omega^{2}$ & b & $i(x-i v)$ us $\left(S_{x}-i S_{x}\right.$ \\
\hline$r$ & 1 & -1 & $\omega$ & - & نמר & $\omega^{2}$ & $b$ & orl/2. 1/2: \\
\hline$\Gamma_{s}$ & 1 & -1 & $-\omega^{2}$ & $\omega=$ & $\omega$ & ע- & $b$ & $\phi(1 / 2,-i / 2)$ \\
\hline$\Gamma_{6}$ & 1 & -1 & -1 & 1 & -1 & 1 & a & $o(3 / 2,3 / 2)$ or $0(3 / 2,-3 /$ \\
\hline
\end{tabular}

$\omega=\exp 1=1 \cdot 3:$

TABELA C12 - TABELA DE MULTIPLICAÇAO PARA O GRUPO $C_{3}$.

\begin{tabular}{|c|c|c|c|c|c|c|}
\hline$r$ & $\Gamma_{z}$ & $r_{z}$ & $\Gamma_{t}$ & $\Gamma_{t}$ & $\Gamma$, & . \\
\hline \multirow[t]{5}{*}{$\Gamma_{:}$} & $r:$ & $r_{3}$ & $r_{4}$ & $\Gamma_{s}$ & $\Gamma_{0}$ & $\therefore$ \\
\hline & $r_{:}$ & $\Gamma:$ & $r_{0}$ & $\Gamma_{\text {. }}$ & $\Gamma_{\xi}$ & $\Gamma$ \\
\hline & & $r_{z}$ & $\Gamma_{\xi}$ & $\Gamma_{0}$ & $r_{4}$ & $r_{3}$ \\
\hline & & & $r_{2}$ & $r_{3}$ & $\Gamma_{3}$ & r. \\
\hline & & & & $\Gamma_{3}$ & $r_{2}$ & \\
\hline
\end{tabular}

TABELA C13 - TABELA DE COMPATIBILIDADE COM O GRUPO DAS ROTAÇÕES PARA O GRUPO C 3 .

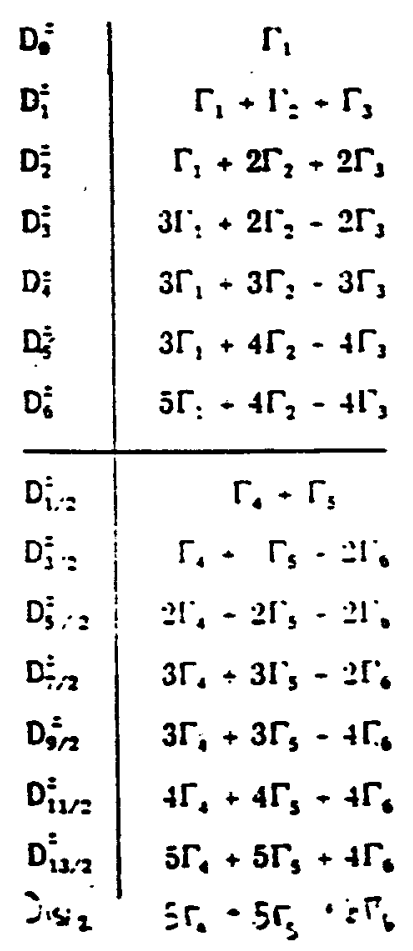

